Medictef Practitioners
Inibrary

$$
\begin{gathered}
\text { Diseases } \\
\text { of the } \\
\text { Intestines } \\
\text { Einhom }
\end{gathered}
$$


(4)

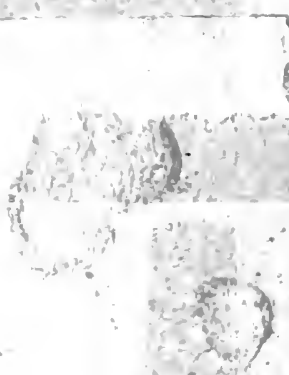

Digitized by the Internet Archive in 2007 with funding from Microsoft Corporation
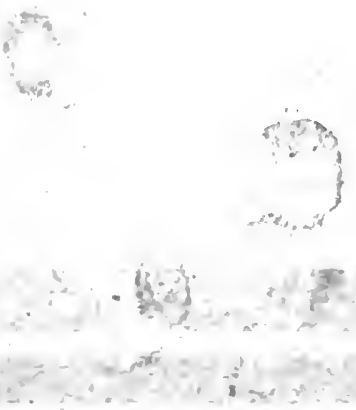

http://www.archive.org/details/diseasesofintest00einhiala 
$111+.90$. 



\section{DISEASES}

OF

\section{THE INTESTINES $\cdots$}

\section{A TEXT-B00K F0R PRACTITIONERS ANI STUDENTS OF VEIICINE}

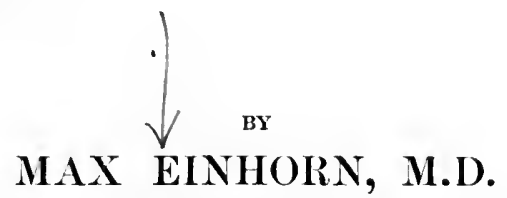

PROFESSOR OF MEDICINE at THE NEW YORK POST-GRADUATE MEICAL ACHOOL AND HOSPITAL, AND VISITING PHYSICIAN AT

$\therefore$ THE GERMAN DISPENSARY, NEW YORK

NEW YORK

WILLIAM WOOD AND COMPANY 

MY ESTEEIED FRIEND AND TEACHER

ERNST VON LEYDEN, M.D.

PROFESSOR OF MEDICINE IS THE INIVERSITY OF BERLIN

THIS BOOK

IS RESPECTFLLLY DEDICATED 


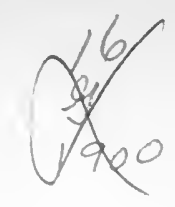

\section{PREF A CE.}

This treatise is a continuation of $\mathrm{my}$ work on "Diseases of the Stomach," the two together comprising the principal disorders of the digestive tract. In discussing the subject of the intestinal affections an effort has been made to follow the same lines laid down in my book on the stomach. The practical points regarding diagnosis and treatment are always placed in the foreground.

Although our knowledge of diseases of the intestines has not made such rapid progress as that of morbid conditions of the stomach, much has likewise been achieved in this field. Surgery has made many successful advances. The elucidation of the intimate relation existing between functional disturbances of the stomach and of the intestines also marks an important step forward, especially as to therapy.

While there are many excellent works on intestinal diseases by German authors, the more recent Euglish literature contains no monographs on this important subject. The medical encyclopedias, it is true, contain very instructive contributions on this topic, and among these Ewald's 
treatise on diseases of the intestines in the "Twentieth Century Practice of Medicine" is a most valuable acquisition. The present rolume, it is hoped, will to a certain extent fill the void in American literature of a monograph on the affections of this portion of the digestive tract. The writer desires to express his indebtedness to Nothnagel, Rosenheim, Boas, Fleischer, Ewald, Pick, Fowler, Treves, and Allingham, whose works have been frequently consulted. He trusts that this book will prove of practical utility to the practitioner, and if it will aid him in more successfully treating this class of cases, the author's effort will be more than recompensed.

Max Ernhorx.

NEW YoRK. April, 1900. 


\section{CONTENTS.}

\section{CHAPTER I}

Anatomy and Pinsiology.

Anatomy,

The Intestinal Canal (Intestinum). . . . . . . . . . 1

The Duolenum, . . . . . . . . . 1

The Small Intestine, . . . . . . . . . . 4

Strueture of the Small Intestine, . . . . 6

The Large Intestine or Large Bowel (Intestinum Crassum), . 11

Histology of the Large Bowel, . . . 17

Physiology, . . . . . . . . . . 18

1. The Secretory Function or the Chemical Processes in the

Intestines, . . . . . . . . . . . 18

2. Absorption. . . . . . . . . . . . 24

3. Motion, . . . . . . . . . 28

\section{CHAPTER II.}

Methods of Examition and Treatmext.

Examination, - . . . . . . . . . . $\quad$. 3 ?

Interrogation, . . . . . . . . . . . . 32

Inspection, . . . . . . . . . . . . 34

Proctoscopy, . . . . . . . . . . 37

Palpation, . . . . . . . . . . 40

Percussion. . . . . . . . . . 44

Auscultation, . . . . . . 4i

Inflation of the Intestine with Carbonic Acid Gas or Air, . 4i

Injection of Water per Anum, . . . . . . . . . 48

Lavage of the Bowel, . . . . . . . . . 45

Examination of the Freces, . . . . . . . . . 49

Treatment, . . . . . . . . . . . 74

Diet, . . . . . . . . . . . . 74

Mechanical Procedures, . . . . . . is

Injections, . . . . . . . . . . is

Massage aud Gymnastic Exercises, . $\quad . \quad$. 80 
Mechanical Procedures:

Iydrotherapy,

Electricity,

CHAPTER 111.

acute and Chronic Intestixal Catarih.

Acute Intestinal Catarrh, . . . . . . . . . 83

Synonyms, . . . . . . . . 83

Detinition, . . . . . . . . . . . . 83

Etiology, . . . . . . . . . . . . . . 83

Morbid Anatony, . . . . . . . . . . . . . $\quad$. 85

Symptomatology, . . . . . . . . . . . . . 86

General Subjective Symptoms, . . . . . . $\quad$. 87

Objective Symptoms, . . . . . . . . 88

Fever. $\quad . \quad 89$

Localization of the Catarnal Process, . . . . . 89

Duration, . . . . . . . . . 90

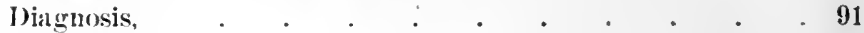

Progrosis, . . . . . . . . . . . . 91

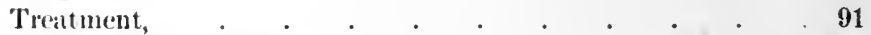

Chronic Intestinal Catarrh, . . . . . . . . . . . 94

Synomyms, . . . . . . . . . . . . 94

Detinition, . . . . . . . . . . 94

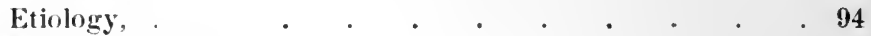

Morbil Anatomy, . . . . . . . . . . . 95

Symptomatology, . . . . . . . . . . 98

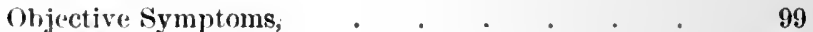

Course,

Diagnosis,

Prognosis, . . . . . . . . . . 104

Treatment, . . . . . . . . . . 105

IIy (Irotherapeutic Measures, . . . . . . . . 106

Mineral Waters, . . . . . . . . . 107

Medicaments, . . . . . . . . . . 107

\section{CHAPTER IV.}

DYsentery.

Dysentery,

Synonyms,

Definition,

Etiology,

Morbid Anatomy, 
Dysentery :

PAGE:

Symptomatology of Acute Dysentery, . _ . . . . 119

Symptomatology of Chronic Dysentery, . . . . . 122

Course, . . . . . . . . . . 123

Complications, . . . . . . . . . . . . . . 123

Jiagnosis, . . . . . . . . . . . . 125

Prognosis, . . . . . . . . . . . 125

Treatment of Acute Dysentery, . . . . . . 125

Treatment of Chronic Dysentery, . . . . . . . 126

CHAPTER V.

UlCERS OF THE INTESTINES.

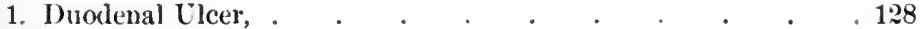

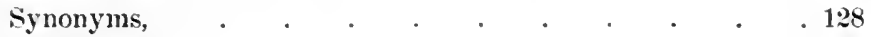

Definition, . . . . . . . . . . . . 128

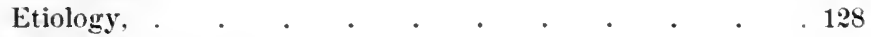

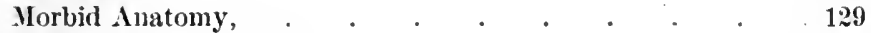

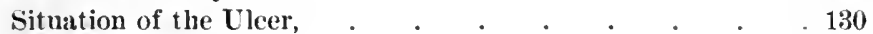

Symptomatology, . . . . . . . . . . . . 131

Course, . . . . . . . . . . . . . . 133

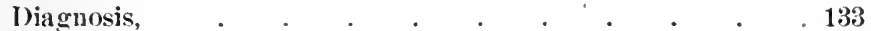

Prognosis, . . . . . . . . . . . . . 134

Treatment, . . . . . . . . . . . . . . 134

2. Embolic and Thrombotic Ulcers, . . . . . . . 135

Pathological Changes, . . . . . . . . 13\%

Symptons, . . . . . . . . . . . . . 136

Diagnosis, . . . . . . . . . . . . . 138

Prognosis, . . . . . . . . . . . . 138

Treatment, . . . . . . . . . . . . 139

3. Amyloid Clcers, . . . . . . . . . . 140

Diagnosis, . . . . . . . . . . . . 141

4. Tuberculous Ulcers, . . . . . . . . . . . 141

5. Syphilitic Uleers, . . . . . . . . . . . 144

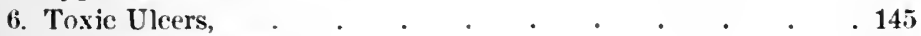

Symptomatology, . . . . . . . . . . . . . . . . . . . . . .

Diagnosis, . . . . . . . . . . . . . 147

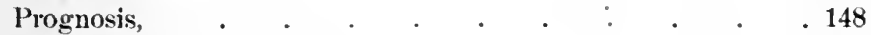

Treatment, . . . . . . . . . . . . . . 148

CHAPTER VI.

Neoplasms of the Intestine.

Malignant Growths,

Cancer, 
Cancer:

Definition, . . . . . . . . . . 150

Etiology, . . . . . . . . . . . . . 150

Location, . . . . . . . . . . 151

Morbid Anatomy, $\quad . \quad$. $\quad . \quad$. $\quad . \quad$. 152

Symptomatology, . . . . . . . . . . . 154

Course. . . . . . . . . . . . . . 163

Diagnosis, . . . . . . . . . . . 16:

Prognosis, . . . . . . . . . . 164

Treatment, . . . . . . . . . . . . 164

Sarcoma and Lympho-Sarcoma, . . . . . . . 166

Benign Tumors of the Intestine, . . . . . . . . 167

\section{CHAPTEIR VII.}

\section{Hevorrhoids.}

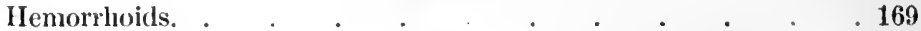

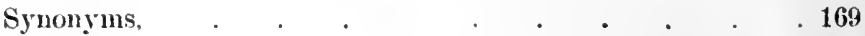

Definition, . . . . . . . . . 169

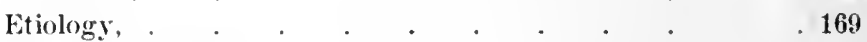

Morbid Anatomy, . . . . . . . . . . . . . 171

Symptomatology, . . . . . . . . . . . . 174

Diagnosis. $\quad . \quad . \quad . \quad . \quad . \quad . \quad . \quad .179$

Prognosis. . . . . . . . . . . . . . 180

Treatment, . . . . . . . . . . . 180

Radical. . . . . . . . . . 185

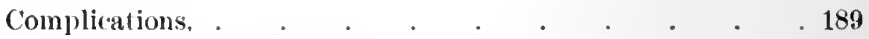

Prolapse of the Rectum. . . . . . . . . 189

Fissure of the Anus, . . . . . . . . . . 193

\section{CIIAP'TER VIII.}

\section{Appendicitis.}

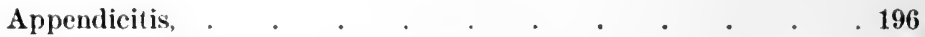

Synonyms, . $. \quad . \quad . \quad . \quad . \quad . \quad . \quad . \quad .196$

Definition. . . . . . . . . . . . . 196

General Remarks, . . . . . . . . . . . 196

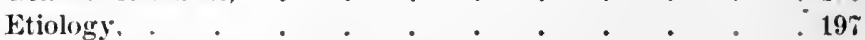

Morbid Anatomy, . . . . . . . . . . . . 202

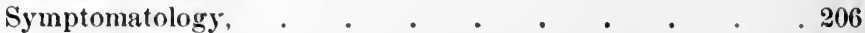

Course, . . . . . . . . . . . . . 208

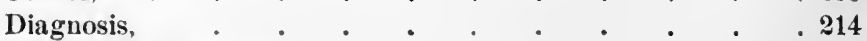

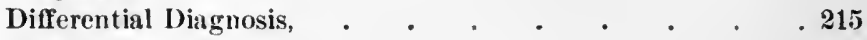

Prognosis, . $\quad . \quad . \quad . \quad . \quad . \quad . \quad . \quad . \quad .216$ 
Appendicitis:

Treatment, Medical, 218 Surgical,

\section{CHAPTER IX.}

\section{INTESTiNal OBstruction.}

Introductory Remarks, .226

Acute Intestinal Obstruction, . . . . . . . . . . . . 227

Synonyms,

Definition,

Etiology,

Compression of the Intestine, . . . . . . . . . 227

Strangulation by Adhesions, . . . . . . . . 228

Strangulation by Meckel's Diverticulum, . . . . 230

Volvulus,

Obturations.

233

Intussusception, . . . . . . . . . . . 234

Pathological Changes, . . . . . . . . . . . 236

Symptomatology, . . . . . . . . . . . . . . 238

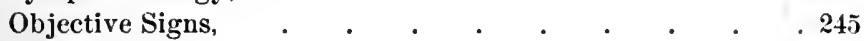

Course, . . . . . . . . . . . . . . . 24 .

Diagnosis, . . . . . . . . . . . . . 249

Prognosis, . . . . . . . . . . . . . . 258

Treatment, . . . . . . . . . . . . 258

Medical, . . . . . . . . . . . 258

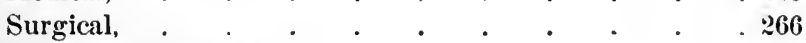

Chronic Intestinal Obstruction, . . . . . . . . . . . 268

Etiology, . . . . . . . . . . . . . . . 268

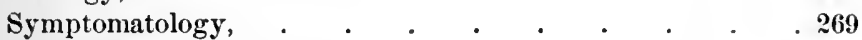

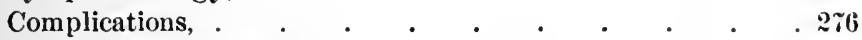

Course and Prognosis, . . . . . . . . . . . 2ir

Diagnosis, . . $\quad . \quad . \quad . \quad . \quad . \quad . \quad .2 \pi$

Treatment, . . . . . . . . . . . . 278

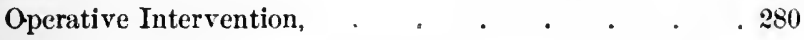

\section{CHAPTER X.}

Nervous Affections of the Intestines.

General Remarks,

Motor Neuroses of the Intestines,

Diarrhoa, . . . . . . . . . . . . . 284

Etiology and Symptomatology, . . . . . . 284

Diagnosis, . . . . . . . . . . . . . 289 
Diarrlıca :

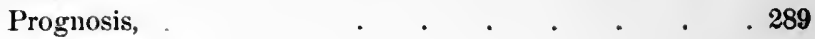

Treatment, . . . . . . . . . . . 289

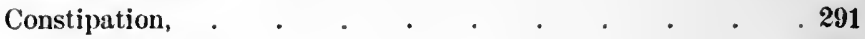

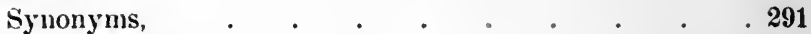

Detinition, . . . . . . . . . . . 291

General Remarks, . . . . . . . . . 291

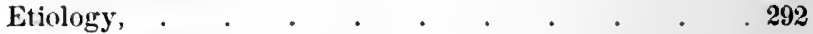

Symptomatology, . . . . . . . . . 297

Diagnosis, . . . . . . . . . . . . . 302

Prognosis, . . . . . . . . . . . 304

Treatment, . . . . . . . . . . 304

Morul, . . . . . . . . . . . 305

Dietetic, . . . . . . . . . 305

Mechanical, . . . . . . . . . . . 306

\section{CHAPTER XI.}

Neirvous Affections of tue Intestines.

Motor Neuroses (Continned), . . . . . . . . . 314

Paralysis of the Intestines, ․ . . . . . . . . 314

Diagnosis, . . . . . . . . . . 315

Treatment, . . . . . . . . . . . . 315

Proctospasmus, or Spasm of the Rectum, . . . . . 316

Diagnosis, . . . . . . . . . . . 317

Treatment, . . . . . . . . . . . 317

Paresis and Paralysis of the Sphincters of the Anus, . . 317

Diagnosis, . . . . . . . . . . . . 318

Prognosis, . . . . . . . . . . . . . 318

Treatment, . . . . . . . . . . . . 318

Peristaltic Restlessness of the Intestines, . . . . . . . 319

Definition, . . . . . . . . . . 319

Etiology and Symptomatology, . . . . 319

Diagnosis, . • • • • • • • • . 320

Prognosis, . . . . . . . . . . . . . 320

Treatment, . . . . . . . . . . . . . 320

Meteorism, . . . . . . . . . . . . 321

Etiology, • • • • • • • • • • . 321

Symptomatology, . . . . . . . . . 322

Diagnosis, . . . . . . . . . . . . 323

Prognosis, . . . . . . . . . . . . 323

Treatment, . . . . . . . . . . 323

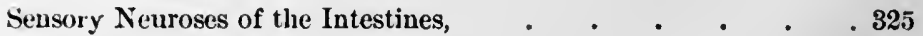

Enteralgia, . . . . . . . . . . 326 
Enteralgia:

Patie

Synonyms, . . . . . . . . . $8: 26$

Definition, . . . . . . . . . . . . 826

Etiology, . . . . . . . . . . . . . . 326

Symptomatology, . . . . . . . . . . . 327

Diagnosis, . . . . . . . . . . 899?

Prognosis, . . . . . . . . . . . 830

Treatment, . . . . . . . . . . . 330

Hypogastric Neuralgia, . . . . . . . . 33:

Treatment, . . . . . . . . . 3932

Hyperæsthesia, Paresthesia, and Anæsthesia of the Intestine, 333

Treatment, . . . . . . . . . 3:34

Secretory Neuroses of the Intestines, . . . . . . . . . . . . . . .

Membranous Enteritis, . . . . . . . . . 335

Synonyms, . . . . . . . . . 335

Definition, . . . . . . . . . . 335.

History, . . . . . . . . . . . . . 835

Etiology. . . . . . . . . . . . . . . 339

Symptomatology, . . . . . . . . . . 341

Diagnosis, . . . . . . . . . . 343

Treatment. . . . . . . . . . . . . 844

Intestinal Neurasthenia, . . . . . . . . . . . 34

Diagnosis. . . . . . . . . . . 348

Treatment. . . . . . . . . . 348

\section{C'HAPTER XII.}

Intestinal Parasites.

General Remarks. . . . . . . . . . . . . 349

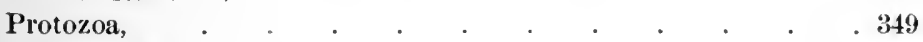

Amebs, . . . . . . . . . . . 349

Sporozoa. . . . . . . . . . . . 350

Infusoria. . . . . . . . . . . . . . 350

Vermes, . . . . . . . . . . . . . . . . . . . .

Cestodes (Tape Worms), . . . . . . . . . 351

General Remarks, . . . . . . . . . 351

Tænia Solium, . . . . . . . . . . . 354

Tænia Saginata or Mediocanellati. . . . . . . 355

Bothriocephalus Latus. Tænia Lata, or Pig Head. . . $35 \pi$

Tænia Nana, . . . . . . . . . 358

Tænia Cucumerina. . . . . . . . . . 358

Tænia Flavopunctatu or Trenia Diminuta, . . . . 359

Treatment, . . . . . . . . . . 359

Trematodes (Fluke Worms). . . . . . . . . 362 
Trematodes (Fluke Worms):

PAGE

Distoma Hepaticum or Liver Fluke, . . . . . . 362

Distoma Lanceolatum, . . . . . . . . . . 363

Distoma Hæmatobium or Bilharzia Hæmatobia, . . 364

Nematodes (Round Worms), . . . . . . . . . 365

Ascaris Iumbricoides (Common Spool or Kound Worm), . 365

Diagnosis. . . . . . . . . . . 367

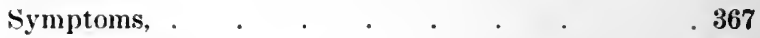

Prophylaxis, . $\quad . \quad$. $\quad . \quad$. $\quad . \quad$. $\quad .368$

Treatment, . . . . . . . . . $\quad .368$

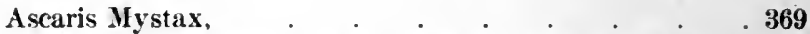

Oxy uris Vermicularis, Awltail, Seat or Pin Worm, Maggot

or Thread Worm, . . . . . . . . . 369

Symptoms, . . . . . . . . . . $\quad .370$

Diagnosis, . . . . . . . . . . 371

Prophylaxis, . . . . . . . . . $\quad .371$

Treatment, . . . . . . . . . 371

Anchy lostoma Duodenale, Dochmius Duodenalis, or Stron-

gylus Duodenalis, . . . . . . . 372

Symptoms, . . . . . . . . . . . $\quad .374$

Course, . . . . . . . . . . . . .

Diagnosis, . . . . . . . . . . 375

Treatment, . . . . . . . . . . . . 376

Anguillula Stercoralis, . . . . . . . . 376

Anguillula Intestinalis, . . . . . . . . . . 376

Trichocephalus Dispar, Whip Worm, . . . . 377

Symptoms, . . . . . . . . . . . 377

Diagnosis, . . . . . . . . . . . $\quad .377$

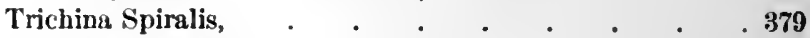

Prophylaxis, . $\quad . \quad$. $\quad . \quad$. $\quad . \quad$. $\quad .380$

Treatment, . $\quad . \quad . \quad . \quad . \quad . \quad . \quad . \quad .380$ 


\title{
DISEASES OF THE INTESTINES.
}

\author{
CHAP'TER I. \\ ANATOMY AND PHYSIOLOGY. \\ ANATOMY. \\ The Intestinal Canal (Intestimum).
}

The intestinal canal may be divided into two parts, the small intestine and the large intestine (Fig. 1). The small intestine (intestinum tenue) is about seven to eight metres long, the first portion being called the duodenum, the second the jejunum, and the third the ileum. With the exception of the duodeuum the small intestine lies for the most part inside the more fixed portion of the large intestine and is connected to the posterior abdominal wall by the mesentery. This broad membrane extends from above downward and from left to right, from the end of the duodenum abore to the ileocreal valve below, enclosing the jejunum and ileum along the whole of their extent.

\section{The Duodernum.}

The duodenum, so called on account of its length (being about twelve inches long), is, unlike the other parts of the small intestine, very definite in position and extent. It is that part which is not suspended by the mesentery. It is, further, the most fixed as well as the widest part of the small intestine, measuring one and one-half to two inches 
in diameter. It has a curved shape, somewhat resembling that of a horseshoe. It surrounds the pancreas and is divided into four parts:

1. The superior horizontal portion (pars horizontalis superior) begins at the pylorus, lying at the level of the first lumbar vertebra, and runs slightly upward and backward toward the right until it reaches the right side of the vertebral column. It ends at the neck of the gall bladder, and is the most movable of the four portions. It is covered by the two layers of the peritoneum which are continued from the stomach, and by these it is completely surrounded. Above it lie the liver (quadrate lobe) and the gall bladder, below it is the pancreas, and behind it are the common bile duct and hepatic vessels.

2. The descending portion of the duodenum, beginning at the neck of the gall bladder, is about twice as long as the first portion, and runs almost vertically to the second or third lumbar vertebra. It lies to the right of the lumbar vertebra, and touches the right kidney. In front of it and crossing it almost at a right angle, runs the transverse colon. It is more fixed than the first portion. On its left side is the pancreas, and the common bile duct a little more posteriorly. Into this part of the bowel, and at its imer and back part, but four inches from the pylorus, the common bile duct and pancreatic duct enter. The portion at which these ducts enter, occasionally forms a small sinus (diverticulum or ampulla Vateri).

3. The third part or the transverse portion is the longest, measuring abont five inches. It extends from the base of the second or third lumbar vertebra on the right side obliquely across the spine to the upper part of the left side, ascending a little on its way. In front of it is found the lower layer of the transverse mesocolon. The superior 
meseuteric vessels cross this part of the duodenum, running between it and the pancreas in order to reach the mesentery. This portion is in relation with the pancreas and superior

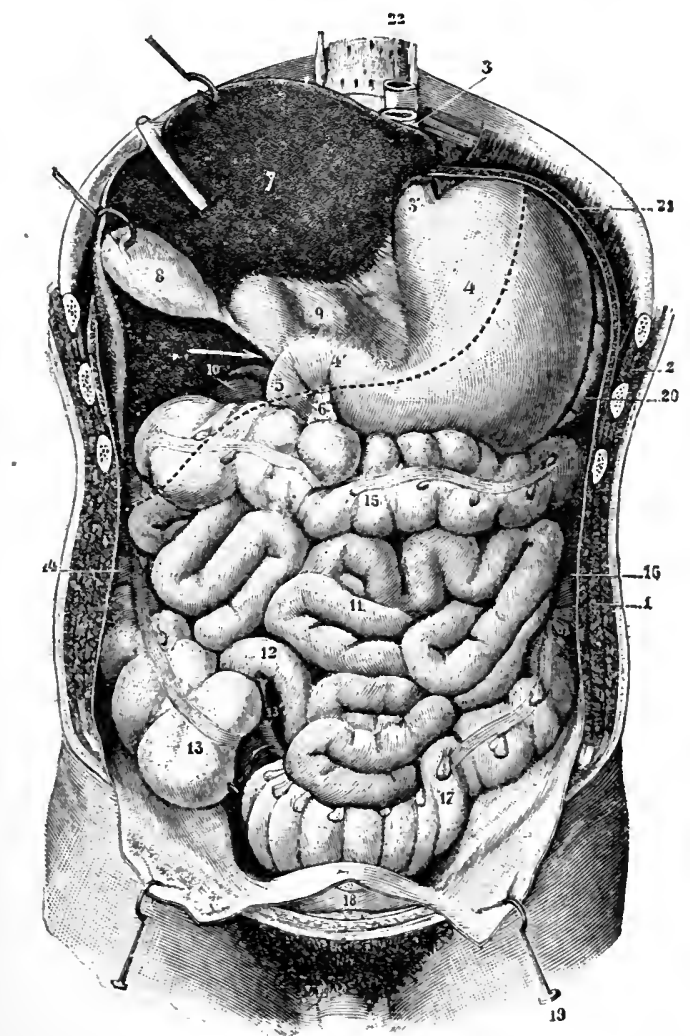

FIG. 1.-The Intestine, as Seen from the Front. after Removing the Omentum (Testut). 1. Abdominal wall; 2, wall of the thorax : 3 , asophagus : $3^{\prime}$, cardia : 4 , stomach ; $4^{\prime}$, pylorus $; 5$, duodenmm $; 6$, pancreas $; 7$, liver $; 8$, gall bladder: 9 , gastrobepatic ligament; 10 , right kjdney and its suprarenal capsule; 11 , small intestine; 12, terminal portion of the 11 enm; 13 , ctecum : $133^{\prime}$, its appendix : 14 , ascending colon : 15 , transverse colon; 16 , descending colon ; $1 \%$. ileopelvic colon : 18 , bladder ; 19 , parietal peritoneum : 20, spleen : 21, diaphragm: 22, thoracic aorta.

mesenteric artery above, with the vena cava, aorta, and crura of the diaphragm behind. It is the most fixed portion of the duodenum. 
4. The fourth part of the duodenum or second ascending portion ascends vertically at the left side of the spine. It is about one inch long and forms the end of the duodenum. It is firmly fixed in its place by the musculus suspensorius duodeni, the latter being the name of the fibrous band, containing some plain muscular fibres which descend to the vertical part of the duodenum from the left crus of the diapluragm and the tissues about the coliac axis. It terminates at this point in the jejunum, forming the flexura duodenojejunalis at a place situated to the left of the second lumbar vertebra.

\section{The Small Intestine.}

The small intestine which forms the continuation of the duodenum is composed of the jejunum and ileum. There is really no marked structural difference between the two, and it is therefore hardly possible to determine where one ends and the other begins. As a rule, the upper two-fifths are designated as the jejunum and the lower three-fifths as the ileum. The jejuno-ileum fills the greater part of the abdomen. It occupies the umbilical, hypogastric, iliac, and lumbar regions, and is more or less encircled by the large intestine. The coils formed by the jejunum and ileum are very movable and completely inrested by the peritoneum. They are supported and attached to the posterior parietes by the mesentery. The latter extends from the end of the duodenum to the ileocrecal junction. The point at which the mesentery is attached above is on a level with the lower border of the pancreas and just to the left of the vertebral bodies. From this point of insertion the mesentery follows an oblique line running downward and to the right, crossing the great vessels and ending in the iliac fossa. The length of the mesentery from the spine 
to the intestines varies in different parts of the canal, its average being eight to nine inches. It soon attains its full length, and within one inch of the end of the duodenum is already six inches long. The small intestine hangs on the mesentery in the form of coils, and the folds which the mesentery forms may be compared to those of a fan.

The small intestine including the dnodenum has an average length of about twenty feet. The calibre of the small iutestine is larger at its upper end and gradually diminishes in size until its entrance into the large bowel. Thus at the beginning the jejunum has a calibre of $17.5 \mathrm{~cm}$., the ileum at its beginning of $11.5 \mathrm{~cm}$, and at its end 9.5 $\mathrm{cm}$. The ileum passes perpendicularly into the ascending part of the larger bowel just above the cæcum, its mucosa forming a double valve, called valvula Bauhini. The jejunoileum is the most movable part of the intestinal tract. Wherever a free space is left it occupies it. It is therefore most often met with in hernias. During gravidity or when a tumor or ascites exists in the abdomen the small intestine moves up higher and thus escapes compression.

The small intestine receives its blood supply from the abdominal aorta. The arteria gastroduodenalis, a branch of the arteria hepatica, supplies the upper part of the duodenum; the lower part of the duodenum and the jejunum and ileum are supplied by the arteria mesenterica superior. The latter vessel branches off into a fine net of numerous small vessels which run through the intestinal wall. The end ramificatious penetrate the submucosa and here again form a net. From the latter the finest ramifications penetrate the mucosa and form a capillary system of the villi and glauds. The venous blood flows partly into the vena gastrica superior, partly into the vena mesenterica superior, and empties itself into the vena porta. The lymphatics 
form a continuous series which is divided into two sets, that of the mucous membrane and that of the muscular coat. The lymph vessels of both sets form a copious plexus. They run between the two folds of the mesentery and end in the mesenteric lacteals. They are provided with valves which prevent the current from flowing backward, the direction of which is into the truncus lymphaticus intestinalis and finally into the thoracic duct.

The nerres of the small intestine originate principally from the plexus mesentericus superior or the sympathetic. The duodenum is supplied by the plexus hepaticus, a branch of the plexus ceeliacus. The abdominal part of the vagus, namely, the plexus gastricus, anterior and posterior, also supplies the small intestine with nerves. The nerres, which are mostly non-medullary, enter the intestinal wall in connection with the branches of the arteria mesenterica . superior and form a subserous net. They then penetrate the long muscular fibres and form between these and the circular muscular fibres ramifications which consist of numerons groups of multipolar cells (plexus mesentericus seu Auerbachii.) ; fine brauches of nerves arising here supply the muscularis. Others penetrate the circularis, reach the submucosa, and form the submucous nerve plexus, containing small groups of gauglion cells (Meissuer's nerve plexus) ; fine ramifications also supply the muscularis mucosæ, the muscles of the villi, and end in the remaining part of the mucosa.

\section{Structure of the Small Intestine.}

The small intestine is composed of four principal coats: the serous, muscular, submucons, and mucous (Fig. 2). The serous coat is formed by the visceral layer of the peritoneum. The muscular coat consists of an internal circular 
and an external longitudinal layer (Fig. 3). The former is usually considerably thicker than the latter. They both

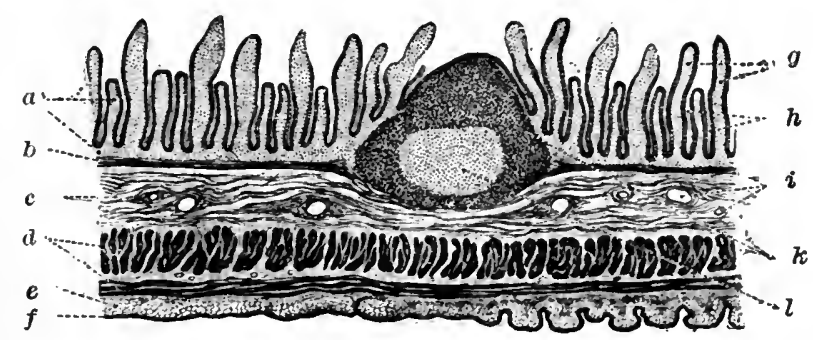

FIG. 2.-Longitudinal Cross-section through the Wall of the Small Intestine (lleum). Solitary lymph nodules (nodnlus lymphaticus solitarius). Intestinal glands (Lieberkuehni) (Toldt). $a$. The uncous layer; $l$, the muscularis mucosa : $c$, che submucons layer; $d$, the musinlar layer ; $\ell$. the subserosa ; $f$, the serous layer : $y$, intestinal villi: $h$, intestinal glands (Lieberkuehn) : $i$, blood-vessels ; $k$, a solitary lymph nodule ; $l$, its centre.

consist of bundles of unstriped muscular tissue supported by connective fibres. The submucous coat consists of connective tissue in which numerous blood-ressels and lym-

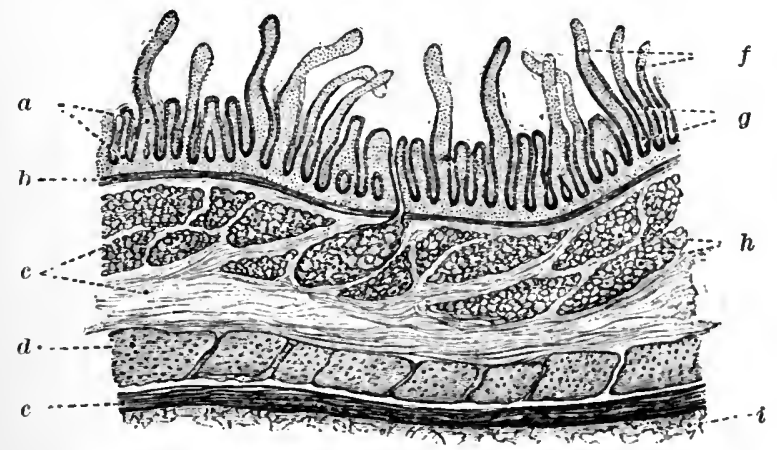

Fig. 3.-Longitudinal Cross-section through the Wall of the Duodenum. Brunner's glands (glandula duodenales) (Toldt). a, The mucous lnyer; $l$, the muscularis mucosæ: $c$, the submucous layer: $d$, the circular muscular layer; $e$, the longitudinal muscular layer; $f$, intestinal villi ; $g$, intestinal glands (Lieberkuehn); h, Brunner's dnodenum glands : $i$, serous layer.

phatics ramify. The mucous membrane is the most important coat with regard to the function of digestion. It 
consists of a very thin muscular layer (muscularis mucosæ) containing circular and longitudinal fibres, the tunica propria of the mucosa, a tissue made up principally

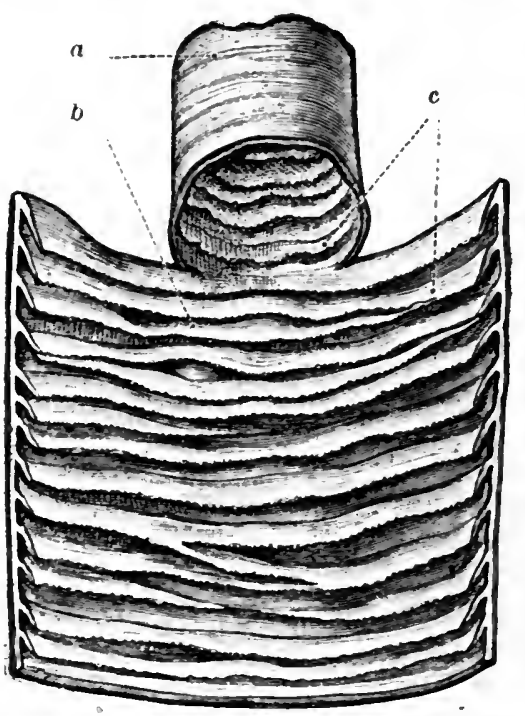

FIG. 4.-Jejunum Partly opened (Toldt). $a$, Serosa ; $b$, mucosa : $c$, circular folds of Kerkring.

of reticular connective tissue with numerous leucocytes, glands, and the epithelial covering. The mucous membrane of the small intestine is of a grayish-red color and has a velvety appearance. It possesses certain large folds of valvular flaps (valvulæ conniventes Kerkringi) (Fig. 4). These are permanent crescentic folds of mucous membrane set transversely to the long axis of the intestine. Each one extends from one-half to two-thirds of the distance of the lumen. The largest are more than two inches long and about one-third of an inch wide. They begin somewhat below the pylorus, are very large just below the entrance of the bile duct, remain conspicuous until the middle of the jejunum is reached, then become smaller and gradually disappear at the lower part of the ileum. They serve to increase the surface of the mucous membrane.

The microscopical anatomy of the mucous membrane reveals the following: The entire inner surface of the small intestine is composed of villi, certain papilliform processes, and glands; an epithelial layer containing columnar epi- 
thelial cells with a striated border, and some goblet cells cover the entire surface. The villi are formed principally by elevations of the tunica propria of the mucous membrane (Fig. 5). They are about 0.5 to $0.7 \mathrm{~mm}$. in height and about 0.1 to $0.2 \mathrm{~mm}$. wide and number almost ten millions. Each villus possesses a centrally located space for chyle which is covered with endothelial cells and comuected with the lymphatics of the intestinal mucosa. Each villus contains a perfect arrangement of blood-ressels and muscular fibres which originate in the muscularis mucosæ. When filling up with blood each villus expands, while under the contraction of its muscle it shrinks. Thus it is enabled to perform the function of suction and pumping. The villi form the main organ for the absorption in the small intestine.

Around the villi lie their glands. First, there are tubular glands (of Lieberkïhn), and, secondly, acinous glands of Brumner. 'The former' are similar in structure to the tubular glands in the stomach. They cover

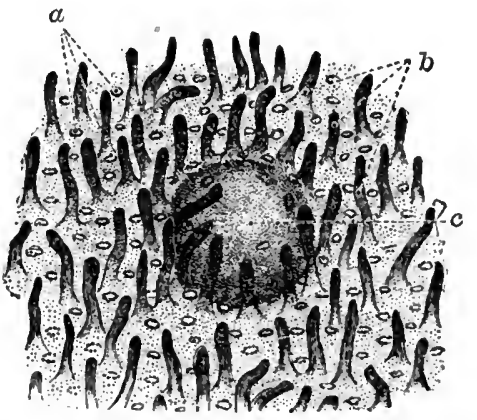

FIG. 5.-Mucous Membrane of the Ileum with a Solitary Lymph Nodule (Toldt). $a$, Intestinal glands (Lieberkuehn): $b$, intestinal villi : $c$, a solitary lymph nodule. almost the entire surface of the whole small and large intestine. Each glandular tubule is about 0.3 to $0.4 \mathrm{~mm}$. long and opens without forming any ramifications. They number over forty millions and form the principal organ of intestinal secretion. Brunner's glands are confined to the duodenum. They are most abundant at the commencement of this portion of the intestine, diminishing gradually as the duodenum 
advances. They are situated beneath the mucous membrane and embedded in the submucous tissue. Each gland is a branched and convoluted tube lined with columuar epithelium. In structure they are very similar to the pyloric glands of the stomach, but are more branched and convoluted, and their ducts are longer. The duct of

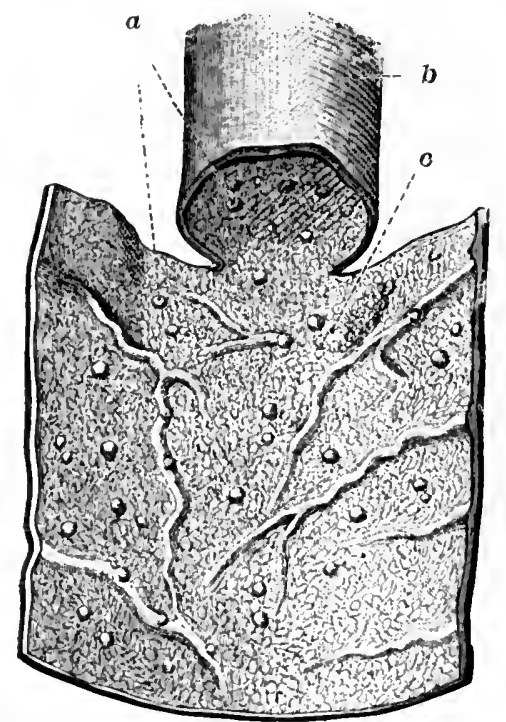

FI(t. B.- -lleum Partly opened (Toldt). $a$, Solltary lymph nodules; $h$, serosa; $c$, mucosa. each gland passes through the muscularis mucosæ and opens on the surface of the mucous membrane.

Solitary follicles or glands are found scattered throughout the mucous membrane of the small intestine. They are most numerous in the lower part of the ileum. Each one has a diameter of from 3 to $6 \mathrm{~mm}$. The structure of the solitary follicle is similar to that of the lymph nodes and consists of a dense retiform tissue packed with lymph corpuscles and permeated by fine capillaries. There are no ducts. The interspaces of the retiform tissue are continuous with larger lymph spaces at the base of the gland, by which they communicate with the lacteal system. The base of the nodules is in the submucous tissue. It penetrates the muscularis mucosæ and enters the mucous membrane forming a slight projection of its epithelial layer. The solitary. follicles are the breeding place of the lymph cells. They 
are met with in two conditions, namely, either scattered singly, in which case they are termed glandula solitarix (Fig. 6), or aggregated in groups varying from one to three

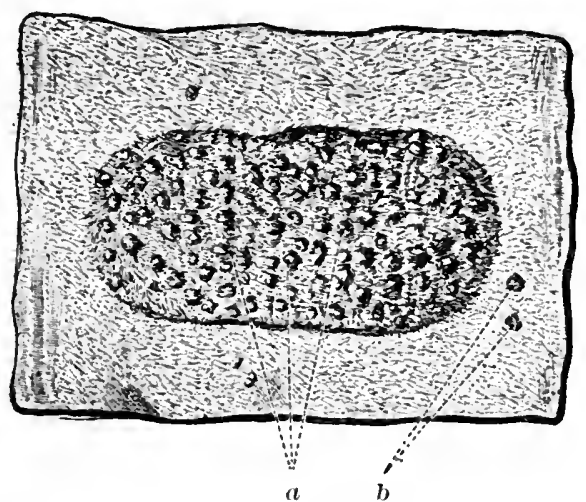

FIG. 7.-Peyer's Patch (Noduli Lymphatici Aggregati) in the Ileum (Toldt). $a$, Peyer's patch : b, solitary lymph nodules.

inches in length and about one-half inch in width. The surface of the solitary follicles is free from villi. Chiefly of an oval form, their long axis is parallel with that of the intestine. In this state they are called glandulre agminatro or Peyer's patches or plaques (Fig. 7). They are almost always placed opposite the attachment of the mesentery. Peyer's patches number about twenty to twenty-eight. In some cases they are already found in the jejunum, but they are most prevalent in the ileum.

The Large Intestine or Large Bouel (Intestinum Crassum).

The large intestine extends from the termination of the ileum to the anus. It is about five to six feet in length. Its calibre decreases from beginning to end except at the ampulla of the rectum where it is larger. It measures $28.5 \mathrm{~cm}$. in circumference at the junction of colon and cæ- 
cum, $20.5 \mathrm{~cm}$. at the end of the ascending portion, $14.5 \mathrm{~cm}$. in the descending portion. The large intestine is divided into the crecum, colon, and rectum. With the exception

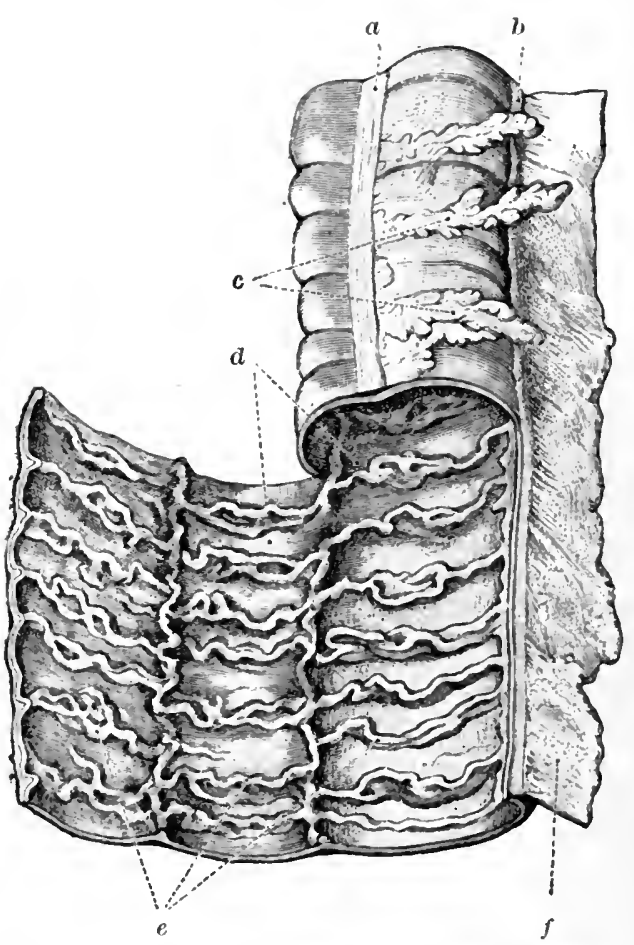

Fir. 8. -The Large Bowel Partly opened along the Sesentery (Toldt). a, Free taenia: $h$, temia mesocolica: $c$. appendices epiplolece; $d$, the mucosis; $e$, the semilunar folds of the colon: $f$, the mesocolon. of the rectum it possesses three trenir, these being groups of nonstriated muscular fibres ruuning lengthwise with the lumen of the intestine. Between the trenixe the walls are somewhat sacculated. The circular muscular fibres are also accumulated in spots, leaving short interrals between each other, thus forming constrictions and

expansions (haustra coli) across the intestiue (Fig. 8). The large bowel is further characterized by appendices epiploicx, external ponches, formed by the peritoneal covering containing fat. The crecum is the head of the colon or that part of the large bowel situated below the mouth of the ilenm (Fig. 9). It lies in the right iliac fossa and is 
completely covered by the peritoneum. In the filled condition it touches the anterior abdominal wall. Starting from the inner and back portion of the crecum lies the processus vermiformis or appendix, forming a narrow, somewhat bent, blind-ending tube. The appendix is movable and has its own mesentery (mesenteriolum). Its length varies between 2 and 20 em. and its width between 0.5 and $1 \mathrm{~cm}$. The appendix opens into the crecum (ostium processus vermiformis), occasionally forming a crescentic fold (valvula processus vermiformis). In man it coustitutes an entirely functionless organ which occasionally give's

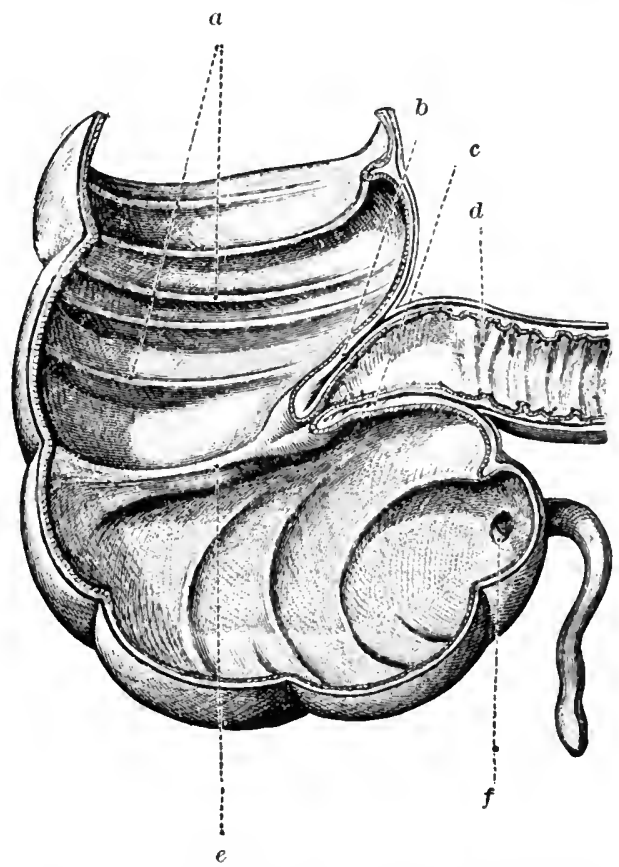

FIG. 9.- Section of the Cremn and Ileum, showing the Entrance of the Latter into the Cacum ('loldt). $a$, The semilunar folds of the colon; $b, c$, the fleocreal valves $(b$, the upper, and $c$, the lower one): $a$, the end portion of the ileum: $c$, the posterior ileocreal valve: $f$, the appendicular valve: $q$, the appendix.

rise to manifold

ailments. The appendix has no fixed position. J. D. Bryaut " found it most often "inward," then "behind the cecum," "downward and inward," "into the true pelvis."

'J. D. Bryant: Annals of Surgery, February, 1893, p. 164. 
Without distinct demarcation the creum merges into the ascending colon. It passes vertically above the crest of the ileum and runs along the posterior abdominal muscles and the lower part of the right kidney. At this point just in front of the kidney and immediately beneath the liver the colon bends toward the left of the flexura coli dextra. The ascending colon is posteriorly adherent through connective issue with the parts just mentioned, while the peritoneum covers only its anterior and partly also its lateral surfaces. In close proximity to its median wall lies the ascending part of the duodenum. Beginning at the flexura coli dextra the colon runs across the abdominal cavity from right to left (transverse colon), forming the longest segment of the large intestine. It passes from the hepatic flexure in the right hypochondrium transversely and slightly upward from right to left along the anterior abdominal wall to the splenic flexure in the left hypochondrium. This part of the colon is the most movable. It has a very long mesentery, called the transverse mesocolon. The usual position of the transverse colon corresponds to a line separating the umbilical and epigastric regions. It is in relation by its upper surface with the lower part of the liver and gall bladder, the greater curvature of the stomach and the lower end of the spleen; by its under surface with the small intestine; by its anterior surface with the great omentum and abdominal wall; by its posterior surface with the transverse mesocolon; on the right side with the second part of the duodenum, and on the left besides the latter with some convolutions of the small intestine.

The transverse colon does not form a straight line connecting the right and left flexures, but is about twice as long as this line and thernfore forms several curves. In 
the left hypochondrium especially, there is an S-shaped coil. The latter fills out the free space in the left hypochondrium which is left by the stomach in its various states of fuluess. Beginning at the flexura coli sinistra the descending colon runs downward in front of the left kidney and the quadratus lumborum and iliac muscles until it reaches the left iliac fossa. The descending colon runs just in the opposite direction to the ascending colon, and like this is only partly corered by the peritoneum. The descending colon passes into the sigmoid colon or flexure (S Romanum), commencing above the iliac crest and ending below in the rectum at the brim of the true pelvis opposite the left sacro-iliac articulation. It is generally described as an S-shaped curre having an upper colic rim turned toward Poupart's ligament and the lower rectal rim, hanging down into the true pelvis. It has a complete peritoneal covering or mesentery. This part of the bowel is very morable, and its calibre is the narrowest of that of the large bowel. The sigmoid flexure continues into the rectum, forming the terminal portion of the intestinal tube. It runs, coming from the left, in front of the os sacrum down to the bottom of the small pelvis. Only the upper half of the rectum is invested completely with peritoneum (mesorectum) and is attached to the sacral vertebra. The lower half passes between the organs occupying the pelvic floor, being adherent to them by connective tissue. It now runs posteriorly along the os coccyx and terminates in the anus. This part has an incomplete peritoneal covering (plica Douglasii) lying anteriorly and turning backward in order to ascend either over the ragina or the bladder (excavatio recto-uterina, excavatio rectoresicalis). Below this point the rectum has very little mobility as it is covered all around by connective tissue. The en- 
tire rectum is about 18 to $22 \mathrm{~cm}$. long. Its calibre varies. It is widest at the apex of the prostate, forming the ampulla of the rectum.

The longitudinal muscular fibres of the rectum are not arranged in trenix as in the colon, but pass all around the lumen. The circular muscular fibres become more dense from above downward and increase to such a degree at the anal opening that they here form a thick ring (musculus sphincter ani internus). A short distance above this muscle there is also an accumulation of circular muscular fibres (musculus sphincter ani tertius). At the anus the walls of the rectum are connected with striated muscular fibres (sphincter ani externus and levator ani), which are both of importance in the act of defecation.

The colon is supplied by the three arteria colicx, branches of the arteria mesenterica superior and arteria mesenterica inferior. The arteria colica sinistra originates from the arteria mesenterica inferior, while the arteria colica media and superior are tributaries of the arteria mesenterica superior. The veins accompany the artery and empty partly into the vena mesenterica superior, partly into the vena mesenterica inferior. The lymphatics of the colon are uumerous and lie below the glands and all through the submucosa. The plexus mesentericus superior, a branch of the plexus cœliacus, provides the nervous supply of the crecum, ascending colon, and the right half of the transverse colon. The plexus mesentericus inferior, a branch of the plexus aorticus abdominalis, supplies the left half of the transverse colon, the descending colon, and the sigmoid flexure.

The rectum is supplied by the arteriæ hæmorrhoidales superior, media, and inferiores, branches of the arteria mesenterica inferior and arteria pudenda communis. The 
renous blood of the rectum is carried to the venæ hæmorrhoidales principally into the vena mesenterica inferior, thus emptying into the vena portarum, partly, however, into the vena iliaca interna. In this way there is a separate communication (ontside of the portal circulation) with the remaining. vessels of the abdomen. The lymphatics of the rectum form a wide net, running partly to the glands lying behind the rectum, partly to the plexus lumbalis sinister. The nerves supplying the rectum originate from the sympathetic, being branches of the plexus mesentericus inferior, the plexus sacralis (nervi hæmorrhoidales inferior and medii), and the plexus hypogastricus superior.

\section{Histology of the Large Bowel.}

The large bowel consists, like the small bowel, of four coats: the serosa, muscularis, submucosa, and mucosa. The structure of these four coats corresponds to that of the small intestine, except that the longitudinal muscular fibres are arranged in three groups (tæniæ) running along the wall, as mentioned above. The mucosa of the large bowel differs from that of the small intestine in that there is an absence of the folds of Kerkring and of the villi. Lieberkühn's glands are here somewhat longer and sometimes curved.

The mucous membrane of the rectum is thicker, more red, and succulent than that of the colon. There are numerous folds. One conspicuous fold is found 6 to $7 \mathrm{~cm}$. above the anus (plica transversalis recti). In the neighborhood of the anus the folds take a longitudinal direction, and are called columnæ Morgagnii seu recti. The lower region of the rectum contains the epithelial cells of the rectum, pavement-like epithelium, forming a gradual 
transition from the mucous membrane of the digestive tract to that of the external skin. The upper portion of the rectum corresponds exactly to that of the colon.

\section{PHYSIOLOGY:}

The intestines are entrusted with the important office of digesting the food which has not been acted upon by the stomach, of absorbing it, and finally of eliminating the undigested remuants. In order to fulfil this object they have three functions, the secretory, absorbent, and motor. All these functions are supervised by ganglionic cells and nerves, the latter also transmitting sensory impressions.

1. The Secretory Function or the Chemical Processes in the Intestines.

As is well known, the intestiual secretion consists, first, of the bile; secondly, the pancreatic juice; and thirdly, the intestinal juice proper (succus entericus). The composition of each of these and their properties may be found in the text-books on physiology, and also briefly in my book on "The Diseases of the Stomach." It will not be amiss, however, to describe here more fully their joint action in the intestiual canal.

The effect of each of the digestive juices is influenced by that of the others. For this reason the chemical processes in the intestines are quite complicated. The carbohydrates, whose couversion into maltose by the ptyalin has been cliecked in the stomach by the free hydrochloric acid, are now, after reaching the intestines, further changed by the diastase of the pancreatic secretion into maltose, which is further converted into glucose. Cane sugar is likewise converted into grape sugar, while milk sugar, according to 
Voit and Lusk, ${ }^{1}$ remains unchanged. The finer parts of the cellulose also undergo some changes, but their ultimate products are not known. It is certain, however, that under the influence of micro-organisms they partly undergo fermentation, giving rise to the formation of marsh gas, acetic acid, and butyric acid.

The pancreatic juice forms the principal factor of all the digestive processes in the intestinal canal. Besides its action upon the carbohydrates through its diastatic ferment, it acts upon fats by means of the steapsin and upon albuminates by means of the trypsin ferment. According to Nencki ${ }^{2}$ and Rachford, ${ }^{3}$ the fat-splitting action of the pancreas is greatly increased by the presence of bile. The splitting of the fats into fatty acids and glycerin is of greatest importance for absorption. The fatty acids combine with the alkalies of the intestinal and pancreatic juices and form soaps which are either absorbed as such or promote the absorption of fats. There is no doubt that the greater amount of fats taken in with the nourishment is absorbed as a fine emulsion in the formation of which the soaps take part. These processes of fat emulsification, by the action either of the pancreatic juice or of soaps, take place only in alkaline media. If the intestinal contents are acid, emulsification does not occur, or does so only at those places at which the fat comes in contact with an alkaline secretion covering the mucous membrane. According to Claude Bernard ${ }^{4}$ and Dastre, ${ }^{5}$ the action of bile greatly increases the emulsifying property of the pancre-

1 Lusk : Zeitschr. f. Biologie, Bd. 28, p. 275.

2 Nencki : Arch. f. experimentelle Path. u. Pharm., Bd. 20.

${ }^{3}$ Rachford: Journal of Physiology, vol. 12.

"Claude Bernard: "Leçons de physiologie expérimentale," 2d edition, 1865.

'Dastre: Arch. de Physiologie, Tome 2, p. 315. 
atic juice. While the bile exerts a deleterious influence upon the action of pepsin in artificial solutions, its presence in the stomach does not seem to have any inhibitory effect. The bile exerts an influence upon the digestion of the albuminates in the intestines by precipitating the pep$\sin$ in tho acid gastric contents. It thus destroys the action of the pepsin. This precipitate, formed by the gastric contents and the bile, is soon dissolved, partly through the intervention of freshly secreted bile in abundance, partly through the sodium chloride which arises after the neutralization of the gastric juice by the alkalies present. The action of the bile upon the pancreatic digestion of albumin is not deleterious, and may have a beneficial effect in the presence of organic acids which, as a rule, exist in the upper parts of the small intestine.

Aside from the chemical processes caused by the enzymes in the intestines there also exist fermentative and putrefactive changes produced by micro-organisms. These are but very slight in the upper part of the intestine and increase in intensity toward the end of the small intestine and in the greater part of the large bowel, while they again decrease in the lower part of the bowel and in the rectum. According to Macfadyen, Nencki, and Sieber, ${ }^{2}$ who have repeatedly analyzed the intestinal contents of a man with a fistula situated near the end of the ileum, only fermentative processes take place within the small intestine. The contents obtained in this case had a golden-yellow color and showed an acid reaction, the acidity amounting to one per mille. As a rule, they were odorless. The principal elements of the acidity consisted of acetic, lactic, and paralactic acids, volatile fatty acids, succinic acid, and

${ }^{1}$ Macfadyen, M. Nencki und N. Sieber: Arch. f. experimentelle Pathol. u. Pharm., Bd. 28, p. 311. 
bịliary acids; albumin, peptone, mucin, dextrin, sugar, and alcohol were present; leucin and tyrosin, however, were absent. Thus, according to these authors, fermentative processes in the small intestine result merely from the action of microbes upon carbohydrates, which action ultimately leads to the formation of ethyl alcohol and the organic acids just mentioned. The latter prevent the putrefaction of albuminates within the small intestine and also partly check the decomposition of the carbohydrates.

The putrefaction of the albuminates takes place in the large intestine, the contents there having an alkaline reaction. The decomposition of the albuminates by the putrefactive processes caused by micro-organisms goes much further than that by the pancreatic digestion. The pancreatic digestion of the albuminates gives rise to albumoses and peptones, lysin, lysatinin, proteinchromogen, amidoacids, and ammonia. In the putrefaction of the albuminates at first the same products are formed, but the decomposition advances still further and generates a host of new products: indol, skatol, paracresol, phenol, phenyl-propionic acid and phenyl-acetic acid, para-oxyphenyl-acetic acid, hydroparacumaric acid, volatile fatty acids, carbon dioxide, hydrogen, marsh gas, methyl mercaptan, and sulphuretted hydrogen. In the putrefaction of gluten neither tyrosin nor indol is formed while glycocoll is dereloped. Of the products of decomposition just named some are of great importance, as they are eliminated by way of the urine after their absorption from the intestinal wall. Some of them, as for instance the oxy-acids, appear unchanged in the urine, others (like the phenols) after further oxidation, and still others (like indol and skatol) after combination with ethereal sulphuric acids. The presence of ethereal 
sulphuric acids in the urine is thus to a certain extent an indication of the amount of putrefaction going on in the intestine. The putrefactive processes in the intestine relate not only to the ingested food but also to the secretions rich in albuminates. Thus Müller ${ }^{1}$ observed that Cetti during his fasting period first showed a diminution of the amount of indican in the urine which entirely disappeared on the third day. The phenol elimination was also at first diminished, but beginning from the fifth day of fasting it commenced to increase, and on the eighth or ninth day reached an amount which was three to seven times that of a mas under ordinary conditions.

The putrefactive processes within the intestines, however, do not reach that height which they attain outside of the body. Thus, for instance, the fresh contents of the large bowel do not present so fetid an odor as a pancreatic. infusion or decomposing albumin would reveal after long standing. The putrefaction within the intestine is partly checked by several factors:

1. Carbohydrates as such exert an inhibitory influence upon putrefaction (Hirschler ${ }^{2}$ ); the organic acids which develop during their fermentation also partly check putrefaction. Of other foods, milk and kumyss, according to Schmitz, ${ }^{3}$ likewise lessen the processes of bacterial decomposition, this effect being dne to the presence of lactose and also of lactic acid.

2. The bile exerts a decidedly anti-putrefactive action. As shown by Lindberger " and Limbourg, albumin to which bile is added does not decompose so thoroughly as

1 Müller : Berl. klin. Wochenschr., 1887, No. 24.

Hirschler : Zeitschr. f. physiol. Chemie, Bd. 10, p. 306.

3 Schmitz : Zeitschr. f. physiol. Chemie, Bd. 17, p. 401.

4 Lindberger: Maly's Jahresber., Bd. 14, p. 334.

${ }^{5}$ Limbourg : Zeitschr. f. physiol. Chemie, Bd. 13. 
without it. The biliary acids, moreover, inhibit putrefaction through their acid elements.

3. Absorption. The rapid absorption of finids from the intestinal wall and the forward motiou of the contents do not permit the putrefactive processes to get the upper hand.

These fermentative and putrefactive processes taking place within the intestines serve to augment the various means at the disposal of the organism to utilize or to break up into simpler components the more complex groups of various food substances. In the normal state these putrefactive processes are most probably checked before any deleterious substances can be developed.

The intestinal contents on their long way from the duodenum to the anus show the presence of different gases. These consist of traces of oxygen and a larger amount of nitrogen; the latter is derived either from swallowed air which has come from the stomach, or from pure nitrogen which has been diffused from the tissues through the intestinal walls. Carbonic-acid gas is present which has been developed through neutralization of the acid gastric contents by the pancreatic and intestinal juices, and also from the butyric and lactic acid fermentation of the carbohydrates. Hydrogen is found in larger amounts after a milk diet and only in small quantities after a pure meat diet. Methyl mercaptan and sulphuretted hydrogen are present in traces, and undoubtedly owe their origin to the albumin. Marsh ${ }^{l}$ gas likewise results from the decomposition of albumin, but it is also evolved from the fermentation of carbohydrates, especially of cellulose. These different gases are formed and absorbed all along the intestinal walls; and most probably help to mix the contents and thus facilitate absorption. If present in too large quantities, they are easily passed through the rectum; occasion- 
ally some of the gases contained in the upper part of the small intestine may be eructated by way of the stomach through the mouth.

In passing through the large bowel the intestinal contents become thickened through the rapid absorption of the fluids, and at last are eliminated as fecal matter. This (fæces) comprises the remnants of the undigested material, excretory products of the intestines, and a host of microorganisms. The quantity of fecal matter within twentyfour hours varies greatly according to the mode of nourishment. Thus after a mixed diet it amounts usually to from 120 to $150 \mathrm{gm}$. After a vegetable diet, however, the quantity, according to Voit, ' reached $333 \mathrm{gm}$. The reaction of the freces is varied. Often it is found acid in their inner parts, while the outer surface shows an alkaline reaction. Their peculiar odor is principally due to Brieger's skatol, but'also to indol and other substances. Their color is usually of a light or dark brown, according to the character of the nourishment.

\section{Absorption.}

The object of digestion is to dissolve and partially change the food substances into such combinations as can be assimilated by the blood. Before assimilation can be effected absorption must take place. The main place for the absorption of nutritive material is the small intestine. It will be best to describe the process of absorption of the different food materials separately.

(a) The proteids are usually changed into albumoses and peptones before their absorption. Albumen as such, however, is also liable to be absorbed, although not so quickly as when its change into peptone has been accom-

${ }^{1}$ Voit : Zeitschr. f. physiol. Chemie, Bd. 13. 
plished. The absorption of albumoses and peptones takes place through the intestinal wall by way of the capillaries of the blood-vessels and not through the lacteals. Thus Munk and Rosenstein ' observed in a patient with a lymph fistula that after a meal rich in albuminous food the lymph did not contain more proteids than before the meal. The peptones and albumoses do not reach the blood current as such, but are previously reconverted into albumin. This fact has been clearly shown by the experiments of Ludwig and Salvioli. ${ }^{2}$ These investigators tied a resected intestinal coil at both ends and injected into its lumen a solntion of peptone, while the coil was kept alive with defibrinated blood. Although the peptone entirely disappeared from the intestinal coil, the blood did not contain even traces of peptone. It therefore must have become changed into another substance. This change of the peptones into albuminates before reaching the blood is of teleological importance. For, as has been shown by Schmidt-Mühlheim ${ }^{3}$ and others, peptone introduced into the circulating blood is soon eliminated with the urine. Where the change of the peptones into albuminates takes place and by what mechanism are not as yet certain. Some seem to believe that the epithelial cells of the intestinal walls perform this office, others that the leucocytes are the means of its conversion.

The absorption of the albuminates appears to be more complete as regards animal than vegetable food. The reason for this is that the cellulose surrounding the legumen partly renders its absorption more difficult. Again, the peristalsis being greater after vegetable food, the intes-

1 Munk and Rosenstein : Virchow's Arch., Bd. 123.

2Ludwig and Salvioli : Du Bois-Reymond's Arch., 1880, Suppl.

${ }^{8}$ Schmidt-Mühlheim : Du Bois-Reymond's Arch., 1880. 
tinal contents pass through the canal quicker, and thus less of the albumen is utilized. And again, according to Hammarsten,' a part of the nitrogenous substances of the plant proteids appears to be indigestible.

(b) The carbohy drates are absorbed principally as monosaccharides. Glucose, lævulose, and galactose are absorbed as such. Cane sugar and maltose are ordinarily changed first into glucose and lrevulose. According to Voit and Lusk, sugar of milk is not converted, and is either partly absorbed as such or else undergoes lactic-acid fermentation. The different kinds of sugar are absorbed through the capillaries of the villi and thus reach the circulation. They enter the liver through the vena porta and are here retained in great part as glycogen. In case, however, a large quantity of sugar is at once absorbed, it may occasionally reach the lacteals and thus enter the blood current outside of the liver. In such instances sugar appears in the urine, a condition which is known as alimentary glycosuria. The introduction of larger quantities of sugar into the intestinal tract occasionally gives rise to diarrhoea. Carbohydrates, however, even in large amounts in the form of starch, will be absorbed without difficulty and without giving rise.to any trouble.

(c) The fats. In the absorption of fats their emulsification seems to be of greatest importance. Although a small part is absorbed in the form of soaps, the greatest quantity of fat is taken up in the form of an emulsion. The latter comprises not only neutral fats but also fatty acids. These, however, undergo a change into neutral fats after their absorption from the intestinal walls. It is generally accepted that fats after their absorption from the intestinal

${ }^{1}$ Olof Hammarsten : "Lehrbuch der physiologischen Chemie," Wiesbaden, 1895 , p. 293. 
wall directly reach the lymphatics and thus enter the thoracic duct, whence they afterward find their way into the blood current. In a girl with a lymph fistula Mrunk and Rosenstein found that sixty per cent of the ingested fat appeared in the lymph. After giving the patient erucic acid (a fatty acid foreign to the organism) they could discover thirty-seven per cent of this particular substance in the form of neutral fats. Thus it appears to be proven that while the proteids and carbohydrates after their alssorption directly reach the blood current, as mentioned above, the fats are an exception and directly enter the lacteals. The ultimate way in which absorption takes place is not as yet known. It must, however, be accepted that the epithelial cells of the intestinal wall cause this process by some specific action. The absorptive property of the small intestine for fat is very great. According to Rubner, ${ }^{1}$ a man can absorb over $300 \mathrm{gm}$. of fat per day. Not all kinds of fat, however, have the same coefficient of assimilation. Thus fats with a low melting-point (olive oil, goose fat, butter, etc.) are absorbed more (puickly tliau those with a high melting-point (mutton fat and stearin). Moreover, free fats, like butter and lard, are assimilated more quickly and thoroughly than bacon, in which the fat is surrounded by connective tissue.

Besides the above-named three groups of food substances, water and different salts which are kept in solution are very quickly and thoroughly absorbed all along the intestinal tract. Aside from the salts, other soluble substances of the secretory juices are also absorbed. Thus the urine contains traces of pepsin and also urobilin, which shows that the biliary products must have been absorbed and eliminated through the urine. According

${ }^{1}$ Rubner: Zeitschr. f. Biologie, Ba. 15. 
to Schiff,' the bile is absorbed from the small intestine and reaches the liver with the blood current in order to be eliminated again by this organ from the blood.

The pancreatic juice being the principal factor in the digestion of the different kinds of food, it appears of interest to ascertain how much of these foods will be absorbed after the pancreas has been excluded from participation in the act of digestion. Minkowski and Abelmann ${ }^{2}$ experimented on dogs by extirpating the pancreas, and found that fortyfour per cent of the proteids and from fifty-seven to seventyone per cent of carbohydrates (amylaceous food) were absorbed, while the fats remained totally unabsorbed. The fat contained in milk, being emulsified, however, was absorbed to the extent of from twenty-eight to fifty-three per cent.

While the main place at which the absorption occurs is the small intestine, the large bowel is also able to serve-in this capacity. Thus aside from the absorption of fluids and salts which normally takes place in this organ, albuminates and carbohydrates can be absorbed in considerable amounts, and fats in small quantities. This function of the large bowel is of great practical importance, as it is utilized in some conditions for nourishing purposes (rectal alimentation).

\section{Motion.}

The motor function or peristalsis of the intestine has for its objects the thorough mixture of the contents and their propulsion through the entire canal until their final exit through the anus. Nothnagel ' and Braam-Houk-

${ }^{1}$ Schiff : Pflüger's Arch., Bd. 3.

' Abelmann: "Ueber die Ausnutzung der Nahrungsstoffe nach Pankreasexstirpation." Inaug. Dissert., Dorpat, 1890.

${ }^{8} \mathrm{H}$. Nothnagel : "Beiträge zur Physiologie und Pathologie des Darms," Berlin, 1884. 
geest ${ }^{1}$ have studied the process of intestinal peristalsis in animals. After laparotomy the latter were kept in a bath of physiological salt-water solution of $38^{\circ} \mathrm{C}$, and the motions of the intestines were investigated.

Three types of intestinal peristalsis were discerned: 1 . The ordinary peristaltic motion. The intestinal tract contracts at a certain point and thereafter relaxes. The contraction is carried with moderate rapidity for a certain length contiguously in the direction toward the anus and the contents are pushed forward. 2. Oscillating motions. An intestinal coil is here moved to and fro all along its mesentery without any particular contraction at any point. The contents are not propelled, but simply mixed up during these motions. 3. Rotary motions. A filled intestinal coil experiences a circular constriction which is rapidly carried over the intestine for the length of about $20 \mathrm{~cm}$. This is exactly the same process as described under 1 , but executed in a violent manner.

While the first two types of intestiual peristalsis are purely physiological, the third type is partly pathological. It is met with only when the contents are mixed with a great deal of gas. Thus, after indiscretions in diet, we often feel this kind of rapid motion going along with a gurgling sound (tormina intestinorum). This type is observed only in the small intestine, but never in the large bowel.

The small intestine manifests much quicker peristalsis than the large bowel, the motions of which are very slow. Here the haustra during the act of peristalsis contract and then protrude in regular order. The small intestine while empty does not show any motion whatever, but after the entrance of chyme into the duodenum intestinal peristalsis

'Braam-Houkgeest : Pflüger's Arch., Bd. 7; p. 266. 
begins. It is not, however, transmitted down to the Bauhinian valre without interruption, but stops as a rule at a certain distance from its starting-point (about $20 \mathrm{~cm}$.). After an intermission of some duration it begins again. Thus one or more intestinal segments may be in a state of peristalsis while other parts of the intestine in between are at rest. The time for the arrival of the first particles of chyme from the duodenum into the crecum is about two hours. But, of course, the intestinal peristalsis must contiuue until the stomach has expelled the last portions of the chyme, that is to say, within about two hours after the stomach has become empty the small intestine as a rule will also be found free of contents. The forward motion of the contents in the large bowel is a very slow one indeed. It takes as a rule from twenty to twenty-four hours for the fecal matter to move from the cxcum to the rectum.

Antiperistalsis, or reversed motion of the large bowel and the small intestine, beginning at the auus and extending upward, has never been seen by Nothnagel in physiological conditions.

The process of peristalsis is controlled by nervous influences. Anerbach's and Meissner's plexus most probably contain automatic nerve centres for this act. But there are also other centrally located nerrous agencies. Thus after great mental excitement diarrhoea very often results, showing that the intestinal peristalsis must have been greatly increased through the influence of the brain. There are also numerous nerves which supervise the motor function of the entire intestinal tract. Pflüger' has shown that the splanchnic nerve contains inhibitory fibres for the

1 Pfliger: "Ueber das Hemmungs- und Nervensystem fĩr die peristaltischen Bewegungen der Gedärme," Berlin, 1857. 
intestinal peristalsis. According to Ehrmann, ' accelerating and inhibitory fibres supervising intestinal peristalsis are contained in the vagus as well as in the splachnicus, but they have a varied function according to the way they end, whether in the longitudinal or in the circular muscles. The longitudinal muscles are stimulated by the splanchuic and paralyzed by the vagus. The circular muscles, on the other hand, are stimulated by the vagus and paralyzed by the splanchnic.

Normally the chyme acts as a stimulus on the intestinal canal and provokes peristalsis (through the influence of the nerves). Too cold drinks, indigestible food, organic acids (present in too large amount) may often cause an increased peristalsis and thus produce diarrhœea. Toxic substances which are ingested or developed from unwholesome food may have the same effect.

${ }^{1}$ Ehrmann : Wiener med. Jahrbücher, 1885. 


\title{
CHAPTER II.
}

\section{METHODS OF EXAMINATION AND TREATMENT.}

\author{
EXAMINATION.
}

\section{Interrogation.}

THE examination begins with a thorough interrogation of the patient. Before starting with the narration of the present ailment a general outline of previous sicknesses is of value. Diseases which involve the intestinal canal, like $t y$ phoid fever, dysentery, and the like, are of special importance, as they are liable to be etiological factors in the development of consecutive ailments. The mode of living, with regard to habits (drinking, smoking, etc.), should also be inquired into.

The patient is then asked to describe his present complaint. He should state the time when the trouble began and its nature. If the chief complaint refers to pains, it is necessary to inquire as to their location and character. Pains felt in the neighborhood of the narel usually originate in the small intestine; those experienced in the right iliac region often emanate from the appendix; while those in the left iliac region and in and about the rectum have their starting-point in the sigmoid flexure and in the lower portion of the rectum. Are the pains of long duration or do they last only a very short while, a few seconds or minutes? The former variety is usually caused either by an affection of the sensory nerres of the intestines or by some organic lesion, like ulcers, etc. The latter variety, to which 
the name colic is applied, is due to a strong spasmodic contraction of a certain part of the bowel. Colicky pains are often followed and relieved by the passing of flatus or of fecal matter. Occasionally these pains also shift from one place of the abdomen to another, and the route of their travel is distinctly felt by the patient.

Abnormal sensations, a feeling of heat or cold may also be experienced over a certain area of the abdomen. A frequent or constant desire for an evacuation (teviesmus) is encountered in dysentery and in many affections of the rectum. It is also advisable to inquire whether the pains and abnormal sensations appear at a certain period of the day or at a certain time after meals (soon after eating or three to four hours later), or whether they are experienced at night or especially in the early morning hours.

The condition of the bowels should always be described in detail. Do the bowels act regularly and is the evacuation of sufficient quantity? What is its consistency? Is the stool of sausage-shape and pliable, or is it hard or very soft, mushy, watery? What is its color? Is it dark brown or light yellow or clay-colored or black? Is there an admixture of mucus or blood? If there is constipation, inquire whether the bowels move without any cathartics after a period of constipation of a few days, and if not, whether mild aperients are sufficient to cause an evacuation, or whether a strong drastic remedy is necessary. Does the constipation alternate with periods of normal movements or with periods of diarrhoea? Are the periods of constipation, if cathartics are not resorted to, accompanied by any marked symptoms (headaches, dizziness, anorexia, etc.) or not? If there is diarrhœea, the patient should state how many movements a day he has. Is he disturbed during the night, or is the diarrhœe confined principally to the 
morning hours? Does the diarrhœe alteruate with periods of constipation; does it disappear after a change of climate, or is it aggravated by mental excitement? Is there a feeling of exhaustion in connection with it? Is the abdomen filled up with gas (meteorism)? Does this phenomenon pertain to a special part of the abdomen (the upper or lower region, right or left side), or does it extend over the entire abdomen? A feeling of tension in the abdomen with frequent passing of wind, belching, and flatus, is commonly designated as flatulency. It is necessary to inquire whether this symptom is present principally at a certain time of the day or continuously. Absence of flatus is of significance if it occurs in conjunction with obstinate constipation, otherwise it is of no consequence.

In all intestinal disorders it is necessary to inquire as to the state of the stomach. The latter organ being in direct communication with the intestines, it will often be subject to disturbances in intestinal affections. Complaints of a bad taste and smell in the mouth are often made, principally in constipation. Anorexia and nausea are present in the most varied intestinal disorders. Vomiting frequently occurs in intestinal obstruction.

\section{Inspection.}

Inspection of the abdomen is best made in good daylight with the patient in the recumbent posture, but should also be completed by inspection in the standing position. The condition of the skin of the abdomen is first examined. Sometimes striæ or scar-like lines running parallel to each other over some part of the abdomen (especially the lower part), and presenting either a silvery hue or, if not old, a rather reddish tinge, are observed; these are always signs of a very marked former distention of the abdominal pari- 
etes. Thus they are found after frequent preguancies, also after the removal of rapilly developing abdominal tumors, or after tapping for ascites. These striæ persist long after the disappearance of the conditions which caused their derelopment.

Distention of the abdominal veins, giving them a bluish hue, is observed whenever the return flow of the venous blood of the lower extremities is retarded either by increased intra-abdominal pressure (ascites, tumors of the abdomen) or by thrombosis or compression of the iliac vein or of the vena cava inferior. Cirrhosis of the liver and compression of the portal vein often produce the same result. In the latter condition there is an extensive formation of veins over the navel which is commonly called caput Medusæ. After observing the appearance of the skin, the shape of the abdomen is then minutely considered. In normal conditions, in grown people, the abdomen and the chest are on the same level in the recumbent position. In small children the abdomen as a rule is somewhat more prominent than the thorax. In very old age the abdomen appears somewhat sunkeu. The greatest degree of a retracted or trough-shaped abdomen is found in stricture of the esophagus or cardia, in basilar meningitis, and in lead poisoning. Long-continued inanition, no matter of what origin, also causes this phenomenon.

Protrusion of the abdomen occurs either over a definite area or over the entire surface. The abdomen may present the shape of a round hemisphere or of a flattened one if there is an accumulation of air and gas in the intestines (intestinal meteorism). This occurs principally in atonic conditions of the intestines and in hysteria. A uniform protrusion of the abdomen or a general bloated condition is present in general peritonitis, occasionally also in pro- 
nounced atony of the intestines. In case of ascites, no matter to what cause it is due (tumors, cirrhosis of the liver, nephritis, etc.), the abdomen is also more or less evenly protuberant above, while the lower parts bulge out somewhat in the recumbent position. This is caused by the accumulation of fluid in the lower portions of the abdominal cavity. Change of posture alters the shape of the abdomen. This applies to the early period of ascites, during which the abdominal cavity is not yet filled to its maximum; later, when this is the case, the abdomen appears uniformly enlarged, and there is no bulging out of any particular portion. Change of position then no longer alters its shape.

Protrusion of a certain part of the abdomen is noticed in many cases of neoplasm, sometimes in fecal concretions, and occasionally in appendicular abscesses. In umbilical hernia a small, more or less roundish protrusion is noticed in the region of the navel. In diastasis of the rectus abdominis muscles there appears in the middle line of the abdomen a long protrusion of sausage shape consisting of prolapsed intestine. Sometimes there is a pronounced protrusion of this area owing to the escape of a large mass of the bowel through the gap in the muscles.

In patients with thin abdominal walls very small sausage-shaped prominences are occasionally visible which quickly change their configuration, appearing now in one place and now in another. This phenomenon is caused by peristaltic contractions of the small intestine. As a rule, they are not associated with pain and do not denote a morbid condition. Sometimes similar peristaltic waves in the small intestine appear periodically and annoy the patient. Here they may be caused by nervous influences. Peristaltic contractions of the small intestine appearing in a 
violent manner and caused by a stenosis or an obstruction of the intestinal lumen are usually much more pronounced, that is, the prominences are much higher and involve larger areas of intestine, the waves moving with greater rapidity and strength and being accompanied by intense pain. Visible peristaltic contractions of the large bowel

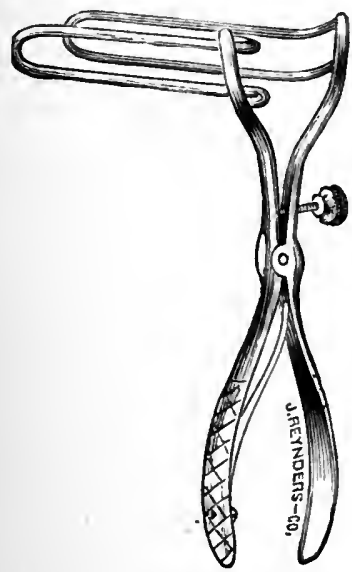

FIG. 10.-Sims' Rectal Speculum.

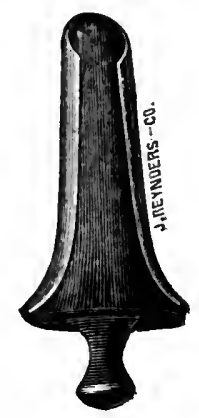

FIG. 11.-Allingham's Rectal speculum.

are ordinarily met with only in cases of partial or total intestinal obstruction.

Inspection of the anal region is best made when the patient lies on his side with his back toward the examiner. The buttocks are held apart with the hands, and thus thorough inspection of the anus is rendered possible. Piles, fissures, fistule may thus be discovered.

Proctoscopy. - In order to inspect the anus internally and also the rectum it is necessary to introduce a speculum. This method of inspecting the rectum is called proctoscopy. Of the many specula devised for this purpose I would mention those of Sims, Allingham, and Kelly as the most practical (see Figs. 10, 11, 12). Kelly's speculum, which 
consists of a hollow metallic tube provided with an obturator, is best suited for this purpose. Before inserting the instrument it must be thoroughly smeared with sweet oil or vaseline. In cases in which the rectal region is inflamed or ulcerated, it is necessary, in order to avoid too much pain, to induce anrsthesia of these parts by painting them with a ten-per-cent cocaine solution or by the introduction

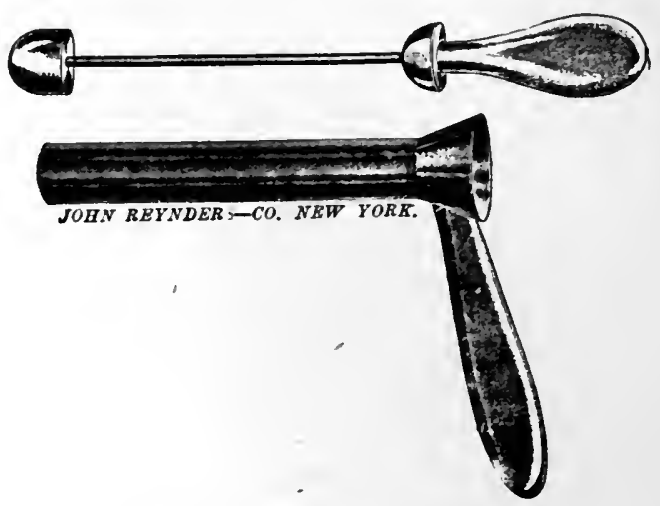

FIG. 12.-Kelly's Rectal Speculum.

of a suppository of opium with belladonna or of cocaine. It is hardly necessary to say that endoscopy of the rectum must not be performed until after a thorough evacuation of the bowels. It is best to wash out the gut before examining with the speculum. When the speculum is in position a portion of the rectal mucosa becomes visible when good light is thrown into the endoscopic tube. The source of light is immaterial, although it is best to have electric light. Usually a small electric lamp with a reflecting mirror fastened to the head of the examiner best serves the purpose. The higher up the bowel has to be examined the longer the speculum must be. After the full insertion of the instrument the highest portion of the bowel is first 
examined, and while gradually drawing out the speculum the entire area of the bowel through which it passes will be brought into view. Small ulcers, atrophic and congested conditious can thus be easily recognized and malignant growths detected at an early period.

Transillumination.-Transillumination of the bowel was first suggested by myself ${ }^{\prime}$ and later practised principally by Heryng and Reichmann. ${ }^{2}$ After a thorough cleansing of the bowel by means of high irrigation about one quart of water is injected and an electric illuminator (very similar in construction to the gastrodiaphane) is inserted into the rectum. The examination must be made in a dark room. By gradually pushing up the instrument successive portions of the bowel may be transilluminated. This method, however, has not as yet proven to be of any practical value.

Roentgen Rays.-The examination of the colon by means of Roentgen rays seems to be somewhat more promising. A soft-rubber-rectal tube through which a flexible wire passes is introduced into the bowel as high up as possible and the patient exposed to the Roentgen apparatus. The wire within the tube becomes visible as a shadow, and thus marks the course of the bowel in which it lies. Inasmuch as it is hardly possible to insert an instrument higher up than the sigmoid flexure, the following procedure for the Roentgen examination appears to be of greater value: The bowel is filled with two quarts of water in which $60 \mathrm{gm}$. (2 ounces) of subnitrate of bismuth are suspended by means of a starch solution. This mixture penetrates almost the entire colon, and thus the position of the large bowel can be determined by the Roentgen rays.

${ }^{1}$ Max Einhorn. "Die Gastrodiaphanie." New. Yorker mediciniscbe Monatsschrift, November, 1889.

${ }^{2}$ Heryng und Reichmann: Therapeutische Monatshefte, 1892. 


\section{Palpation.}

Palpation is the most important procedure available amoug the methods of examination in abdominal diseases. It is best performed in the recumbent position of the patient, the head being slightly raised and the abdominal muscles relaxed as much as possible. In order to effect this the room must be of a comfortable temperature and the hands of the examiner warm. If the patient is fidgety and contracts his abdominal walls, it is necessary to talk to him and to draw his attention away from the examination. I have often noticed a great relaxation of the muscles during an expiration following a deep inspiration. Whenever, therefore, it is difficult to obtain relaxation of the abdomen I tell the patient to take a deep inspiration and then make use of the following period of expiration for palpation. If all these means fail to relax the abdominal muscles, palpation may be tried in a warm tub bath, as first recommended by Chlapowski, or under chloroform narcosis. In cases of great diagnostic importance the latter method is certainly preferable. In palpating the abdomen it is advisable first to examine with the entire palm of the hand, applying very little pressure, thus determining the state and consistency of the abdomen. The hand may thus be passed over the entire abdominal surface from one place to another. This having been done, palpation is then performed with a trifle more pressure, the finger tips being used for this purpose. The latter procedure serves for exploring a more circumscribed area. Finally, deep palpation is practised for which considerable pressure may be required.

Palpation aids us in discovering the position of some of the abdominal organs. With regard to the intestine the 
following portions are often accessible to this method of examination: the crecum and part of the ascending colon, the transverse colon, and the sigmoid flexure. In some instances the descending colon above the sigmoid flexure can also be palpated, especially if it is filled with hard scybala. The jejunum and ileum filling most of the lower part of the abdominal carity (from the navel downward) cannot normally be separately ontlined.

For the detection of tumors in the abdomen palpation is of great service. By means of it we gain information with regard to their size, shape, and consistency. An uneven protuberant surface is characteristic of malignant growths, while an even surface is more often found in benign neoplasms or in intussusception. A fecal tumor can be recognized by indentations made by pressure with the fingers. Sometimes after such pressure it is possible to notice for a moment, when raising the finger, a slipping off of the intestinal wall from the fecal mass. This phenomenon, first described by Gersuny " under the name of "Klebesymptom," I have observed quite frequently and consider of practical value.

Another important object of palpation is to ascertain whether there is tenderness or pain on pressure. While strong pressure exerted upon the intestine through the abdominal wall even normally elicits an unpleasant sensation, there is, however, no distinct pain connected with this act. Tenderness on slight pressure is often present in inflammatory conditions of the bowels and also in ulcerative processes. A circumscribed pain on pressure is present in the appendicular region (McBurney's point) in appendicitis, especially in the acute form. In chronic appendieitis the pain may be elicited only upou very strong press-

${ }^{1}$ Gersuny : Wiener klinische Wochenschrift, 1896, No. 40. 
ure. In ulcerations of the bowel there may be also one or several circumscribed areas very painful to pressure. In pains due to a purely nervous affection of the bowel

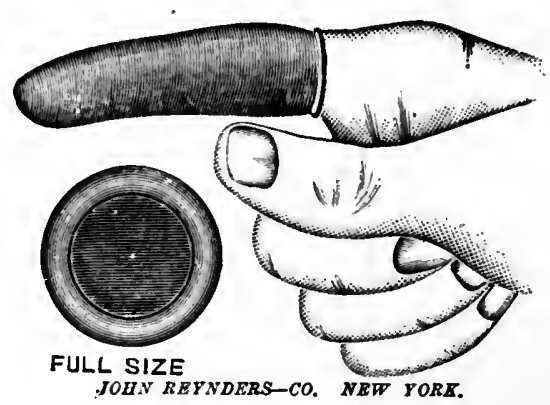

Fig. 13.-Finger Cot. pressure may afford relief. If a mere touching of the abdomen elicits pain, it is a sign either of an extensive inflammatory process within the bowel or of peritonitis.

Palpation in the form of tapping occasionally produces a splashing sound (clapotage) over some portions of the bowel. The splashing sound can be elicited over the colon only when it is filled with liquid or semi-liquid matter and gas. It can be discovered off and on either in the crcum and in the portion of the bowel immediately above it or in the sigmoid flexure. In the small intestine clapotage can be obtained only in the dilated portion of the gut above a stricture. Boas ' first suggested the method of filling up the bowel with from 500 to 600 c.c. of water and then examining for the splashing sound along the colon. When the patient has been thus prepared, clapotage can be produced in the sigmoid flexure; and by having the patient turn on his right side, it can occasionally be produced in the transverse colon, and finally in the creal region. In cases of atony of the bowel Boas was able to evoke the splashing sound even after the injection of only 200 to 300 c.c. of water. Frieden-

'Boas : "Diagnostik und Therapie der Magenkrankheiten, "Theil 1., 1897, 4te Auflage, p. 105. 
wald' has also practised the same method with advantage. Whenever the splashing sound can be produced in the colon it serves the purpose of determining the situation of this organ.

The rectum is best palpated with the index finger well oiled or smeared with vaseline or encased in a rubber cot (Fig. 13) and ancinted in the same way. The condition of the anus and the lower portion of the rectum can be advantageously investigated with the finger. The examination may be made either in the recumbent posture of the patient, in the side or kneeelbow position, or in the standing position. In the latter instance it is well to have the patient exert downward pressure upon. the rectum. Hemorrhoids, polypi, and malignant growths can thus be occasionally discovered. In cases in which there is a suspicion of malignant growths involving portions of the colon not accessible either to palpation by the finger or inspection with the proctoscope, examination with the entire hand in chloroform narcosis can be tried as first practised by Simon." After dilating the anal sphincters, the

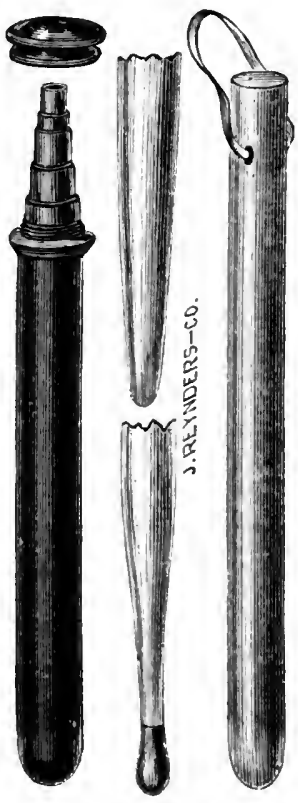

FIG. 14a. Fig. 15. Fig. 14b.

Figs. $14 a$ and $14 b_{0}-$ Cylindrical Bougies.

FIG. 15.-Oiive-Point Bougie. entire right hand and the arm are inserted into the bowel through the anus, and thus the higher portions of the

1 J. Friedenwald : Medical News, 1894.

Simon: Verhandlungen der deutschen Gesellsch. f. Chirurgie, 1871, and Deutsche Klinik. 14\%2. 
colon palpated with the fingers. This method can be recommended only in cases of extreme importance, as such an examination is liable to produce unpleasant symptoms, as, for instance, incoutinence of the rectum, tearing of the mucous membrane, etc.

Palpation of the rectum by means of sounds is performed whenever there is suspicion of a stricture involving portions of the bowel not accessible to examination by the finger. For this purpose either bougies (see Figs. 14 and $15)$, or, still better, rectal tubes of various calibre may be employed. Kuhn' has recently recommended the use of tubes provided with a metal spiral. $\mathrm{He}$ - believes that these penetrate the colon farther up without bending. His statements have, however, not as yet been corroborated.

\section{Percussion.}

Percussion is of less importance than palpation. In many instances it serres to confirm the results obtained by the latter. In percussing the intestines it is best to use the fingers. It should be done rather gently. Mild percussion permits the discernment of slight differences of sound much better than strong percussion. As is well known, percussion over empty intestinal coils or those filled with gas or air gives a tympanitic sound which is londer over the large than over the small bowel. Intestinal coils filled with liquid or solid substances give dulness. In meteorism of the intestines percussion will elicit a tympanitic sound of a deeper pitch than normally, and there will be besides some areas of dulness over the abdomen. The region of the liver and spleen will here show normal conditions with regard to the percussion sounds. Meteorism of the abdomen as a result of perforation will manifest

${ }^{1}$ Kuhn : Deutsche med. Wochenschr., 1897. Nos. 36 and 37. 
an evenly diffused tympanitic sound all orer the abdominal cavity. Usually the dulness orer the region of the liver and spleen will have disappeared. In ascites percussion will reveal an area of dulness in the lower parts of the ablomen, and there will be a change in the character of the sound on altering the position of the patient. Tumors of the intestine give dulness on percussion. Fecal accumulations and appendicular abscesses will also manifest dulness on percussion.

\section{Auscultation.}

Auscultation is not of great significance in diseases of the intestine. Palpation of intestinal coils with the application of moderate pressure may elicit either a gurgling noise or a friction sound. The latter was formerly believed to be pathognomonic of typhoid ferer. Of late, however, it has been recognized that this sign is found in many other conditions. At the time of active peristalsis all kinds of gurgling sounds are heard within the intestine (borborygmi), which, however, are not of much importance. In chronic stenosis of the intestine very loud noises are at times heard, caused by the sudden passage of liquid and gaseous contents through the stricture under great pressure. In the latter affection splashing sounds can also be easily produced over the enlarged bowel abore the stricture. Often a ty mpanitic sound of a metallic character can be heard.

Inflation of the Intestine with Carbonic Acid Gas or Air.

Inflation of the intestine is one of the most important diagnostic procedures. Von Ziemssen,' who first introduced this method of examination, injected successively

1 Von Ziemssen: Deutsches Archiv f. klinische Medicin, 1883, Bd. 33, S. 235. 
into the bowel two solutions, one containing tartaric acid, the other bicarbonate of sodium in water. The carbonic acid gas developing fills the large bowel, which can then be recognized by the tympanitic percussion sound, or, in rare instances, by inspection. Schnetter,' of New York, suggested filling the bowel with carbonic acid gas by means of a tube attached to an inverted siphon containing sodawater, the valve of which is pressed. Here the carbonic acid gas runs into the bowel without any admixture of water. Rosenbach ${ }^{2}$ made use of liquefied carbon dioxide from a sparklet. Instead of the latter Runeberg ${ }^{3}$ recommended inflation of the intestines by means of air. This is best done by a rectal tube to which a compressible air suction bulb is attached. The advantage this method offers consists in the possibility of regulating the amount of the introduced air. In order to be able to measure the amount of insufflated air, Damsch ${ }^{4}$ has recommended the employment of a syringe of kriown capacity. An ordinary bicycle pump can be used for this purpose, the rectal tube being attached to it.

Inflation of the bowel is of importance in detecting a stenosis of this organ. Under ordinary conditions the injected air evenly distends the entire colon, as can be proven by inspection and percussion. In case there is a stenosis in the large intestine the air will distend principally that portion of the bowel below the stricture, while that above will remain unchanged. It is thus possible to recognize the seat of a constriction. The significance of this diagnostic means, however, is confined merely to strictures

\footnotetext{
'Schnetter: Deutsches Archiv f. klinische Medicin, 1884, Bd. 34, S. 638 .
}

O. Rosenbach : Berliner klinische Wochenschrift, 1889, No. 28.

${ }^{3}$ Runeberg: Deutsches Archiv f. klinische Medicin, Bd. 34, S. 460.

-Damsch : Berliner klinische Wochenschrift, 1889, No. 75. 
of a high degree, while a beginning stenosis of the bowel cannot be thus recognized, as the air will pass through it.

The position of the colon can be ascertained by this procedure. Normally the transverse colon is situated somewhat above the navel, while in cases of enteroptosis it may be found about a hand's width above the symphysis.

Inflation of the colon is also of importance in the differential diagnosis of abdominal tumors. As is well known, tumors of the intestine will become more distinct after inflation of the bowel with air, while tumors of the kidney, of retroperitoneal glands, and of the spine tend to recede. According to Minkowski, ${ }^{2}$ abdominal tumors after filling the colon with air or water are usually slightly shifted in the direction of the organ to which they belong.

Inflation of the bowel with air impregnated with ether has been suggested by Dr. Sutton ${ }^{2}$ as a means of recognizing intestinal perforation. For this purpose he makes use of a bottle filled with two drachms of ether. The bottle is provided with a perforated rubber cork to which are attached two rubber tubes provided with stopcocks. One of these is then attached to a bicycle pump and the other to an ordinary rectal tube. The air pumped into the bowel must pass through the bottle containing ether and thus takes up the ether vapors. In case of perforation of the bowel, the ether quickly escapes through the opening into the abdominal cavity and equally distends it; while, if there is no perforation, the bowel, first the large and later the small intestine, becomes filled with air and ether; ultimately the ether reaches the stomach and is usually eruc-

'Minkowski : Berliner klinische Wochenschrift. 1888, No. 31.

${ }^{2}$ E. M. Sutton: "Diagnosis of Intestinal Perforations by Means of Ether Inflation per Rectum." Journal of the Am. Med. Assn., Decem. ber 30 th, 1899 . 
tated. The ether can then be recognized by its characteristic odor. It seems that this procedure is especially useful in gunshot wounds of the abdomen.

\section{Injection of Water per Anum.}

This is done by means of a rectal tube and a fountain syringe provided with a scale indicating the amount of water used. In case of stricture, especially of the lower portion of the colon, the quantity of water which can be injected is not great, while ordinarily from three to five quarts of water can be poured in. Inasmuch às even normally some people are not able to hold large amounts of water in the bowels without experiencing considerable discomfort, the quantity of fluid which can be injected without pain is not of great diagnostic value. Filling up the bowel with water can also be made use of for the determination of the position of the colon, as this organ will then gire a dull sound. For this purpose, however, the procedure in question is not so good as the above-described method of inflation with air.

\section{Lavage of the Bowel.}

Lavage of the bowel in a similar manner as performed in the stomach has been recommended by Boas ' for diagnostic purposes. It is best performed in the lateral posture of the patient after an evacuation of the bowels. The same apparatus as for gastric lavage may be used here. The rectal tube, which represents the stomach tube employed in gastric lavage, is attached to a long piece of rubber tubing provided with a big funnel. The rectal tnbe is inserted as high up in the bowel as possible and then the water is poured in until the patient begins to

'J. Boas : Deutsche Aerzte-Zeitung, 1895. Nos. 2 and 3. 
feel some discomfort. As soon as this is the case the funnel is lowered and thus the water returns. The latter is now. subjected to a thorough examination. Normally the returning water appears pretty clear or slightly turbid by the admixture of small particles of mucus, epithelial cells, and fecal matter. In catarrh of the large bowel a considerable quantity of mucus is found. Ulcerative processes accompanied by hemorrhages or by suppuration are often recognized by the admixture of either pus or blood in the wash-water. Occasionally exfoliated pieces of intestinal mucosa are found in the wash-water, and a microscopical examination of them may be of diagnostic importance.

\section{Examination of the Feces.}

The examination of the frees is of much service in diseases of the intestine. The frecs represent the end product of the digestive act, consisting of residue unsuitable for further assimilation. It is evident that a thorough knowledge of the dejecta will throw light upon the nature of the activity of the intestines.

The normal fæces consist of changed and unchanged remnants of foocl, bacteria, traces of digestive juices, epithelial cells, and salts. The quantity of the freces for twenty-four hours varies greatly with the kind of food taken. In a mixed diet it usually amounts to from four to seven ounces. The color of the freces is usually dark brown owing to changed bile pigment, the bilirubin having become changed in the intestine into urobilin. The diet has great influence upon the color of the freces. Meat produces a dark brown, milk a light yellow color, cacao a more or less brownish-red, huckleberries and claret a dirty black-brown color with a greenish hue. The salts of iron and manganese give rise to a darker color than the usual 
one, while bismuth produces a more or less blackish color. According to Quincke, ${ }^{\prime}$ all these metals are reduced to oxydule combinations which are responsible for these colors, while the former belief that these metals formed sulphides is not correct. Calomel frequently produces a greenish hue, while senna, santonin, gamboge, and rhubarb give rise to an intensely yellow color.

The freces are normally somewhat soft in consistency and have a sausage shape. In abnormal conditions the consistency may be changed in two directions. The dejecta may be greatly hardened and appear in small balls, or in the form of very thin cylinders. On the other hand, the stools may be abnormally mushy or even liquid. The hardened stools which occasionally show grooved impressions from the trenia coli bear testimony to their long sojourn in the intestine, thus being exsiccated from the complete absorption of water. They are, however, by no means characteristic of a stenosis of the intestine. Very soft dejecta may be either watery, as for instance in cholera nostras or asiatica, or they are mixed with mucus which can be easily seen when pouring the dejecta into a glass and inverting it, when the mucus as a rule adheres to the surface of the vessel.

Odor.-The characteristic odor of the fres is normally caused by skatol and also to a less degree by indol. The fecal odor may be increased whenever the freces have been retained much longer than normally in the intestine. On the other hand, freces occasionally present very little or no odor when their sojourn in the intestine has been very short. As a good instance of the latter variety the socalled rice-water movements in cholera nostras and cholera asiatica may be mentioned. Movements with a fetid odor

' Suincke: Munchner medizinische Wochenschrift, 1896, No. 36. 
occur principally in malignant growths of the large bowel accompanied by ulcerative processes.

Remnants of Food in the Freces. - Undigested remuants of food, macroscopically visible, occur in the frecs. Normally, however, only small particles of regetable substances, like potatoes, asparagus, spinach, peas, etc., are found, while remnants of meat can never be discovered with the naked eye. In case particles of meat are visible, it indicates a severe lesion of the intestinal tract. If large amounts of undigested food (even vegetable matter) are present in the fæces, it is also an indication of an existing severe lesion.

Abnormal admixtures frequently occur in the freces, and are occasionally of great diagnostic importance. Thus, blood may be found either in its fresh condition (red) or it may be rery dark but not coagulated. In both instances the blood comes from the lower portions of the large bowel. Sometimes the blood appears in a more changed and decomposed form, giving the freces the appearance of tar. In this instance it originates from the higher portions of the bowels or from the stomach.

An admixture of pus in the dejecta which can be macroscopically recognized occurs only in instances in which pus exists in the lower portions of the large intestine. For if there is pus present in the higher portions of the bowel, it is usually changed before its exit in such a manner that it cannot be detected unless the amount is very considerable.

Fragments of tumor (polypi or torn off particles of cancer) are occasionally found in the dejecta. A thorough examination of these may be of great help in the diagnosis.

Mucus, although a normal constituent of the freces, cannot be discovered in large amounts under physiological 
conditions. Macroscopically visible mucus may exist in the following forms: (1) It may surround the fecal matter in the form of a glassy layer. This usually indicates a diseased condition of the lower portion of the bowel. The mucus may appear in the form of membranes and may be evacuated either alone or after a fecal evacuation. This often occurs in membranous enteritis. (3) The mucus may appear in a mushy movement having a yellowish coloration and be well mixed with freces. If a glass rod is dipped into such an evacuation the mucus adheres to it. (4) The mucus exists in small particles visible with the naked eyo and floating in the watery dejecta. All these varieties of mucus with the exception of (2) indicate the presence of a catarrhal condition of the intestine.

Intestinal parasites also occur in the fæces, and their discovery may elucidate the diagnosis.

Chemical Examination of the Fieces.-The reaction of the frees is normally neutral or slightly alkaline. Under a diet rich in vegetables, however, it is slightly acid. In cases in which there is an occlusion of the bile duct so that it does not empty into the intestines the reaction is strongly acid. The test for the reaction is best made by means of litmus paper. The reaction at the surface of the fecal matter may be different from that in the interior. It is therefore best to test both.

The amount of acidity or alkalinity of the frees can be determined by mixing 10 to 20 c.c. of the fresh fecal matter with about 100 c.c. of distilled water. A drop of a phenolphthalein solution is added and as much of a decinormal solution of either sodium hydrate or sulphuric acid until the red color appears, or if the alkalinity has to be determined, disappears. The reaction of the fæces is, however, not of much diagnostic value. 
Tests for Mucin.-According to Hoppe-Seyler, mucin forms one of the principal constituents of the fæces. In order to test for it the fæces are thoroughly mixed with water and an equal volume of milk of lime, allowing the mixture to stand for several hours. It is then filtered. Acetic acid is now added to the filtrate. In the presence of mucin a precipitate forms. In case particles of suspected mucus are visible within the freces, they can be examined separately in the following manner: A small flake of the mucus is dissolved in a weak solution of potassium or sodium hydrate, and acetic acid added. If the precipitate remains undissolved after the addition of the acetic acid in excess, it prores the presence of mucin. Inasmuch as nucleoalbumin also gives the reaction just described, the positive proof that the precipitate is due to mucin is afforded by heating it in a diluted mineral acid to the boiling-point. If mucin is present the heated solution will contain a substance reducing copper oxide. Another very useful test for the presence of mucin consists in staining the flake of fecal matter resembling mucus with a weak triacid solution (Ehrlich). The presence of mucus pro- duces a green color, while if the flake consists of albumin, a red color arises. This test, first described by Pariser," I have found of practical value.

Albumin. - In order to examine the frees for albumin, they are treated repeatedly with water slightly acidified with acetic acid. The watery extract is filtered several times and the filtrate examined for albumin according to the methods used in examinations of the urine for this substance. The addition of acetic acid and potassium ferrocyanide, however, is best suited for this purpose. Under normal conditions there is no albumin present in the frees.

'Pariser: Deutsche medicinische Wochenschrift, 1893, No. 41. 
Vou Jaksch ${ }^{1}$ found it present in typhoid fever, in isolated cases of acute enteritis, and in chlorosis.

Propeptone and Peptone.-After the test for albumin has been made with negative result, the watery extract of the freces is treated with phosphotungstic acid, the precipitate diluted with water and sodium hydrate and a small amount of a weak solution of sulphate of copper added. A purplish-red color (biuret test) shows the presence of both propeptones and peptones. If it is desirable to ascertain the presence of peptones separately it is necessary to first precipitate the propeptone by the addition of a large amount of ammonium sulphate. In normal dejecta Von Jaksch never encountered peptone. Pathologically he found it in typhoid fever, dysentery, tuberculous ulcer of the intestine, and in perforation peritonitis.

Carbolydiates. - In order to test for the presence of carbohydrates, the freces are subjected to distillation. The residue is extracted with alcolool and ether; the extract is then boiled with water, filtered, and again boiled with the addition of dilute sulphuric acid. This solution is then subjected to Trommer's or Nylander's test for the presence of reducing substances.

In orler to ascertain whether starch is present the watery extract of the frees is examined with Lugol's solution, the presence of starch producing a blue color.

If the dejecta be examined for the presence of sugar, then a watery extract of the fecal matter can be directly tested with the usual sugar reagents. Normally neither starch nor its derivatives (sugar) are found.

Schmidt ${ }^{2}$ suggested testing the watery extract of the fecal matter with regard to the amount of gas developing

${ }^{1}$ Von Jaksch : "Klinische Diagnostik."

${ }^{2}$ Ad. Schmidt : Berliner klinische Wochenschrift, 1898, No. 41. 
through fermentation. For this purpose Schmidt puts the watery extract of the fæces into fermentation tubes (similar to the fermentation saccharometer) and keeps them at blood temperature. The greater the amount of gases developing in the cylindrical part of the tube, the greater the evidence of disturbances within the intestine. The greater proportion of the gas consists of carbonic acid and is due to its formation from the carbolydrates existing in the fecal matter. In order to be able to judge more accurately from this test, Schmidt examined his patients after a certain diet which they had been taking for several days. It consisted of 1,560 c.c. of milk, four eggs, three zwieback, one plate of barley soup, one plate of flour soup, and one cup of bouillon a day. While Schmidt asserts that whenever a considerable amount of gas is found in the fermentation tube this indicates a real disturbauce of the intestine, S. Basch, ${ }^{1}$ who has made a thorough study of Schmidt's method in a considerable number of cases, is of the opinion that on the one hand a considerable amount of gas may be found in cases without any apparent intestinal lesion, and, on the other hand, grave disturbances of the intestine may show a total absence of gas. Inasmuch as Schmidt's fermentation method is certainly complicated and its results are not of great diagnostic value, I do not believe that it will ever come into practical use.

Fat.-The presence of neutral fat and fatty acids is determined in the following manner: The fæces are treated with a considerable amount of ether; the latter is separated and evaporated in a water bath. The fat if present then remains and is visible. In order to show the presence of

'S. Basch : "Welche klinische Bedeutung bezeichnet die Schmidt'scht Gährungsprobe der Faeces?" Zeitschrift f. klin. Med., Bd. 37, Heft 5 and 6. 
soaps which do not dissolve in ether, another portion of fecal matter is uist treated with acids which split up the soaps and then extracted with ether. The quantitative determination of the amount of fat and of its different components is somewhat complicated and of not much service clinically. Those interested in the subject may look up Vou Noorden's "Beiträge zur Lehre voin Stoffwechsel," Heft I., p. 109, Berlin, 1892. Normally fat is never perceptible macroscopically in the fres unless after the ingestion of very large quantities. It may then be visible in small portions of pea size. Pathologically fat may exist in very large quantities in the fecal matter and give it a grayish silvery appearance, the so-called fatty stools. This normally occurs in diseases of the pancreas, and also whenever the absorption by the lymphatics is greatly disturbed.

Blood.-Fresh blood from the lower portion of the intestine, and also from the higher portions of the bowel if present in large amount, is easily recognized by its macroscopic appearance. Often the microscope will reveal well-preserved red and white blood corpuscles. Sometimes, however, the blood is changed to such a degree that it is not easily recognized. Here various tests are required in order to prove its existence, the same procedures being used as for the discovery of blood in the gastric contents. The hæmin test which is chiefly used is made as follows: $\mathbf{A}$ small particle especially suspected of containing blood is dried and powdered and a portion of it put on a slide. A trace of sodium chloride is now added and a drop of glacial acetic acid poured over it and thoroughly mixed. A coverglass is now put over it, the specimen is slowly heated, and after cooling examined with the microscope. The presence of hæmatin crystals shows that there was blood. 
Bile Pigment.-Under normal conditions no unchanged bile pigment is found in the fæees. In catarrhal conditions of the small intestine it has been frequently detected. The presence of bile pigment is ascertained in the following manner: A particle of highly colored fecal matter is brought into contact with a drop of fuming nitric acid. The yellow color usually passes through the various colors of the spectrum-red, violet to green. In some instances a green discoloration appears at once. The test for biliary pigment may also be made as follows: The fæces if liquid are filtered through filter paper, and if not liquid a watery mixture is made and filtered. When the filter paper is dry a few drops of nitric acid are poured on it. The colors just mentioned appear in the form of rings, if bile pigment is present. Still another test is as follows: A small quantity of the fluid dejecta is treated with a concentrated watery solution of sublimate. If the freces contain biliary pigments in considerable quantity, the entire mixture turns green. If, however, the biliary pigment is adherent to certain small fecal particles then these alone turn green.

Biliary Acids. - Whenever biliary pigments appear in the dejecta, biliary acids, as a rule, accompany them. The presence of biliary acids is best revealed by Pettenkofer's test, and is made as follows: A small quantity of fecal matter is thoroughly treated with alcohol, which is then evaporated. To the residue a weak watery solution of bicarbonate of sodium is added, and to this mixture a small quantity of cane sugar and a few drops of sulphuric acid. When biliary acids are present a characteristic red or pink color arises.

Urobilin.-Normally the biliary pigment within the intestinal tract becomes changed into urobilin, which is the principal factor of the characteristic brownish color of the 
freces. The best test for the presence of urobilin is Fleischer's ' procedure which is as follows: A small quantity of freces is put into a test tube and a small amount of alcohol with a few drops of hydrochloric or acetic acid added; the mixture is then left undisturbed for a short time. The presence of urobilin produces a yellow or brown color, the latter, if present in large amount. If the alcoliol is now poured out and a few drops of sodium hydrate added, as well as a small quantity of a chloride-ofzinc solution, there appears, according to the amount of urobilin, a more or less greenish fluorescence in direct rays of light, while in transmitted light the fluid appears pink or yellowish-red. If the watery extract of freces to which some ammonia has been added is filtered and chloricle of zinc added, the presence of urobilin produces a pinkish-red precipitate. If this precipitate is filtered under addition of alcohol containing some ammonia there appears a more or less greenish fluorescence (Schmidt's ${ }^{2}$ test). A small piece of fecal matter is treated with a concentrated watery solution of sublimate and thoroughly mixed with a glass rod. The presence of urobilin gires rise either immediately or a little later to a pinkish-red color, while biliverdin, if present, produces a greenish color.

Normally urobilin is present in the frees. Its absence is observed only in pathological conditions.

Acholic Stool.-The acholic stool presents a grayishwhite, ashy gray, or clay color. It is usually of a soft salvelike consistency. It occurs (1) in conditions in which there is a total absence of bile in the intestine, and (2) whenever the absorption of fat is greatly impaired. Until very recently the grayish-white color has been generally

'R. Fleischer: "Krankheiten des Darms," p. 1160, Wiesbaden, 1896.

A. Schmidt : Verhandlungen des Congresses f. Innere Med., 1895. 
ascribed to the absence of biliary pigments and their modifications (urobilin), but Fleischer and Bunge' have conclusively shown that the whitish color may be observed in fæces containing urobilin, the color being due to the presence of large amounts of fat. In the latter instance the stool, after being treated with large amounts of ether, thus separating the contained fat, assumes a brownish color. This I can confirm also from my own experience.

Ferments.-In order to ascertain the existence of ferments in the freces a glycerin extract of them may be made or the fecal matter may be directly mixed with water containing a small proportion of thymol, and filtered. The filtrate, or the glycerin extract, can now be directly tested for the presence or absence of the different ferments, trypsin and diastase. In order to test for trypsin the fecal filtrate is made alkaline by the addition of bicarbonate of sodium and a few flakes of fibrin are added. The solution is kept at blood temperature for a few hours and then tested with potassium hydrate and a weak solution of sulphate of copper. If trypsin is present, a pinkish-red color will arise in consequence of the peptone which has formed (biuret test). In order to test for diastase, a few cubic centimetres of the filtrate are mixed with about half the amount of a starch solution and kept at blood temperature for half an hour. The mixture is now subjected to Fehling's or Trommer's test for the presence of sugar. Normally, as a rule, these ferments are absent, but in pathological conditions, especially in diarrhœa, they are frequently found.

Concretions.-The frees occasionally contain concretions which may be of diagnostic importance. In order to detect them, especially if they are small, the frees must be

'Bunge: "Lehrbuch der phys. u. pathol. Chemie," Leipsic, 1887, p. 192. 
thoroughly mixed with warm water and poured through a large sieve. While the fecal matter is on the sieve some more water is added and the mass constantly stirred with a wooden stick. Any concretions present will thus be discovered remaining on the surface of the sieve.

The following different concretions may be met with in the freces: (1) Gall stones; (2) pancreatic calculi; (3) enteroliths; (4) coproliths; (5) foreign bodies.

Biliary calculi are easily recognized when they attain considerable size. When they are very small, however, their recognition is somewhat more difficult. The principal constituents of biliary calculi are cholesterin and bile pigment in conjunction with lime.

The small concretions (sand) suspected to be of biliary origin should be examined in the following way: About $2 \mathrm{gm}$. of the mass is well powdered and treated with 20 c.c. of ether, thoroughly mixed and filtered, the filtrate eraporated and tested for the presence of cholesterin in the following manner: (a) Part of the residue is dissolved in hot alcohol and put aside on a porcelain dish for spontaneous eraporation. The precipitate is examined under the microscope. Crystals of rhomboid shape with a ragged edge are characteristic of cholesterin. (b) Another portion of the residue is directly put on a slide, a drop of concentrated sulphuric acid added, and covered with a cover-glass. The cholesterin crystals assume a carmine color at their margins. If now a drop of Lugol's solution is added a violet color arises. (c) Another portion of the residue is treated with hydrochloric acid and a trace of chloride of iron and evaporated. If cholesterin is present a blue color arises. The residue of the original ether mixture is treated with diluted hydrochloric acid, heated, and extracted with chloroform after it has cooled off. The 
chloroform extract is now tested with Mellin's reaction (fuming nitric acid). The presence of bile pigment produces the well-known change of colors.

Pancreatic Calculi.-Pancreatic calculi usually have a rough surface, are brittle, and may be faceted. They are soluble in chloroform and produce on evaporation an aromatic odor (Minich $\left.{ }^{2}\right)$. Bile pigment and cholesterin are absent.

Ehteroliths or calculi formed in the small intestine usually consist principally of inorganic salts (lime, magnesia). They are light in color and ordinarily of small size. They occasionally form after an extensive use of mineral medicaments (lime, magnesia, etc.). They hardly ever give rise to intestinal obstruction.

Coprolittls or fecal calculi are found in the large bowel, principally in places in which there is a retardation in the passage of the frees. Thus they are encountered in the cxcum, in the appendix, in sacculations of the colon, and in the rectum. The coproliths are of stony hardness and of sausage shape. They usually show on section concentric rings. Occasionally they attain considerable size and may give rise to obstruction of the bowel.

Foreign Bodies.-Foreign bodies which have been swallowed may pass through the entire intestinal tract and be eliminated in the fæces. Thus pieces of bone, coins, marbles, needles, and all kinds of foreign substances may be found in the stools. In rare instances concretions of shellac are discovered in the stools of patients who have drunk furniture polish, the shellac forming concretions after the absorption of the alcohol. Hair balls may be found in patients who habitually bite off and swallow hair.

${ }^{1}$ Minich : Berliner klin. Wochenschrift, 1894, No. 8. 


\section{Microscopical Examination.}

The microscopical examination of the frecs is occasionally of assistance in establishing the diagnosis. With Ewald ${ }^{2}$ I do not think it necessary to examine microscopically the freces of every patient presenting intestinal symptoms. In

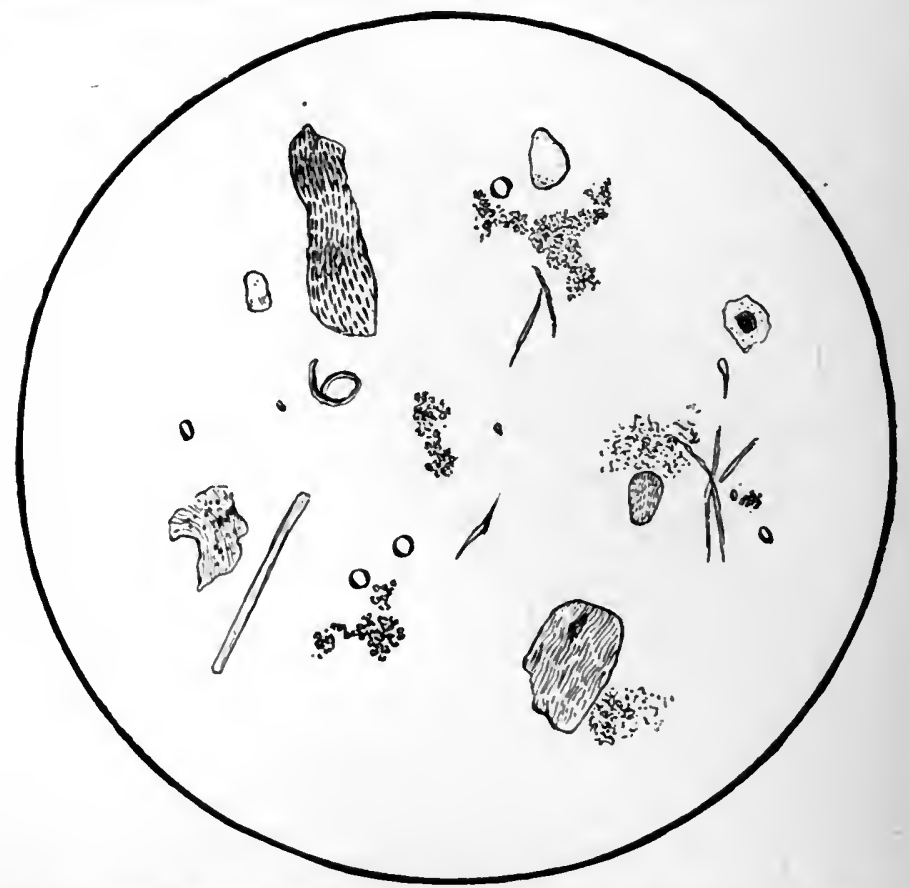

FiG. 16.-Normal Freces, showing a few Fat Crystals and Fat Globules; Digested Muscle and Epithelial Cells: Micro-organisms.

cases, however, in which the diagnosis is not quite clear and the symptoms point to an intestinal lesion, a microscopical examination of the frees should be made.

Diarrhœal stools may be examined under the microscope

'C. A. Ewald: "Diseases of the Intestines." Twentieth Century Practice of Medicine, vol. ix.. p. ?13. 
without any further preparation. Solid fecal matter is examined by taking a small particle of the frees, putting it on a slide, and mixing it thoroughly with a drop of physiological salt solution. In order to avoid the unpleasant odor, a small amount of a watery one-per-cent formalin solution may be first added to the fecal matter. The micro-

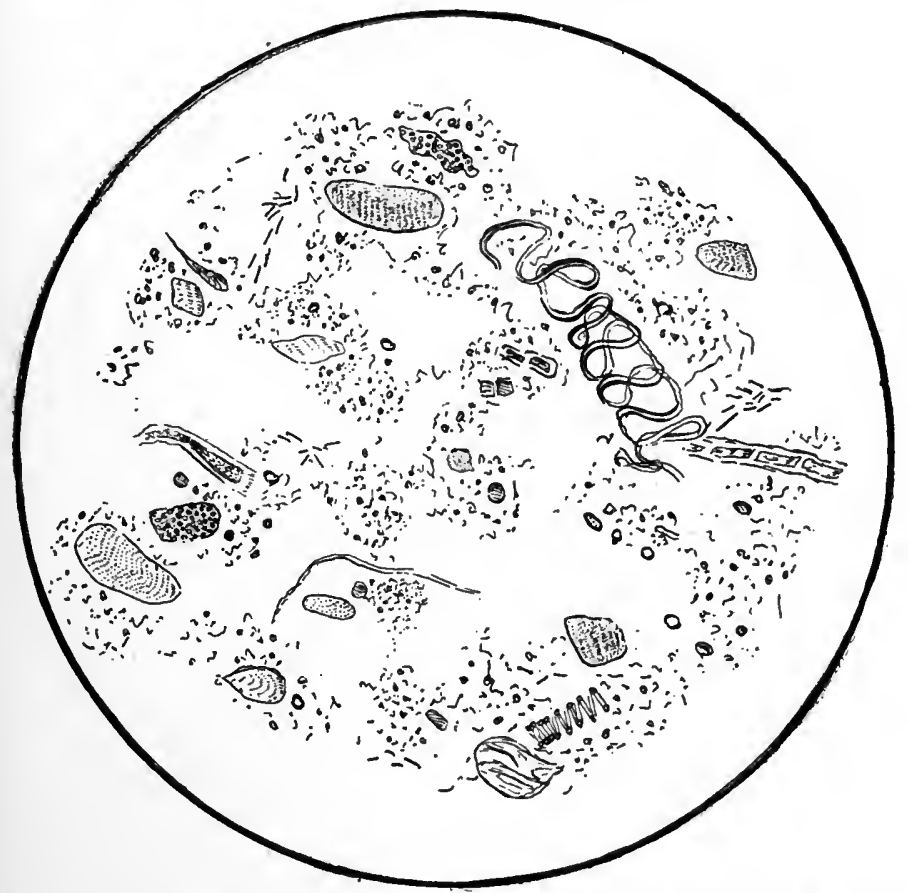

FIG. 17.-Normal Fæces showing Detritus, Plant Cells, Digested Muscle Fibres, Bacteria.

scopic picture of the normal faces varies greatly according to the diet. In people living on a meat diet no regetable residue will be seen, while there will be no remnants of meat in people subsisting on an exclusively vegetable diet. In case of a mixed diet there will be remnants of both in the stool. A mixed diet will reveal the following appear- 
ances: There will be a large number of plant cells, the remuants of various vegetables and fruits. They are usually of considerable size, present peculiar shapes, and can be easily differentiated from animal cells (Figs. 16, 17, 18, 19). The peels of pears and apples and of prunes commonly pass out in the stool entirely unchanged. Notwith-

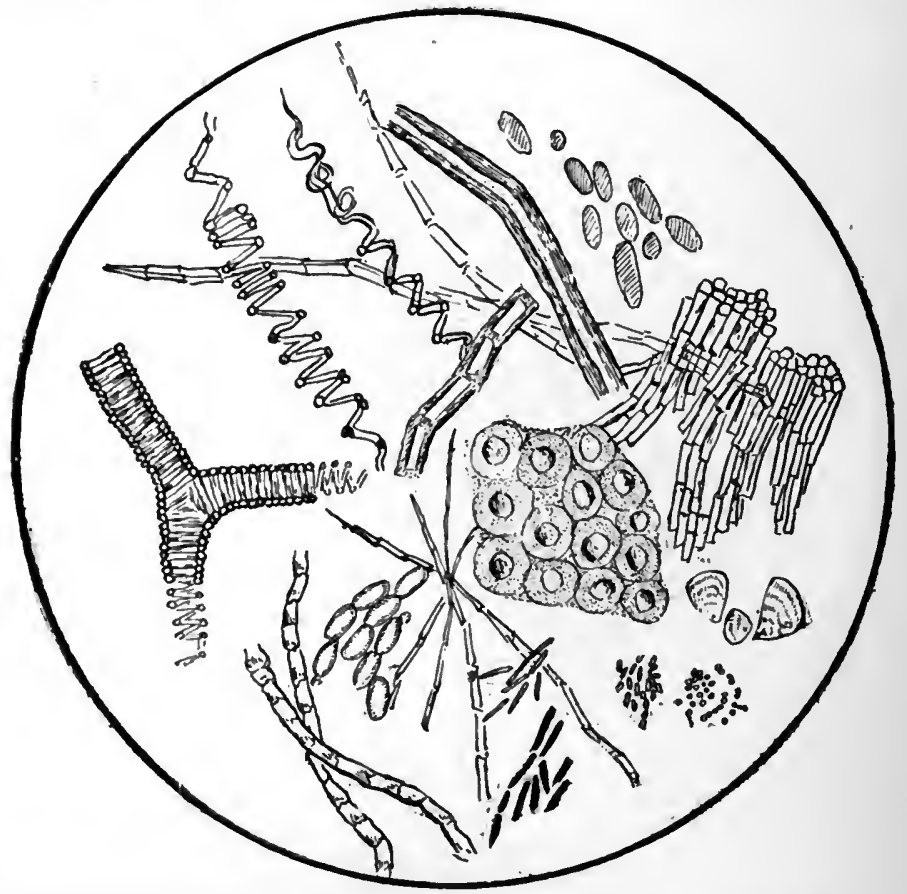

F1G. 18.-Different Varietles of Vegetable Cells found in Normal Faces.

standing the presence of these plant cells in the stools starch, as a rule, is absent. Thus the microscopical specimen when stained with Lugol's solution will show no blue color. If, however, starch appears in a stool in well-preserved granules, it is always pathological, indicating deficient digestion. Minute fragments of meat are found 
in small quantity in the stools. Although considerably changed the muscles can be recognized as such, and the transverse markings can often be noticed. Frequently they present a yellowish tinge from biliary pigment. Connective-tissue fibres and also elastic fibres are occasionally met

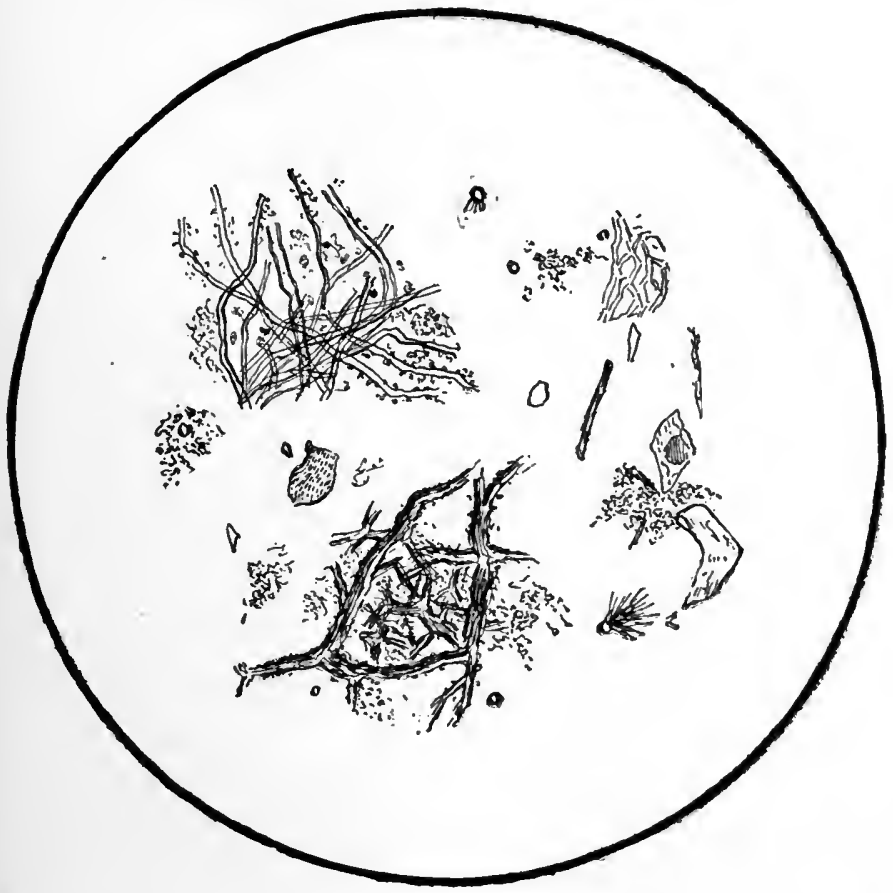

FIG. 19.-Stool of an Hysterical Patient wbo Simulated Passing of Large Quantities of Mucous Membranes in the Faces. The membranes under the microscope showed the structure of common tissue paper; a few plant cells, epithelial cells, and fat crystals were also present.

with, both being quite resistant to the action of the digestive juices. The presence of numerous pieces of meat in the stool is pathological.

Fat.-Microscopically fat can be detected in the fæces in the form of colorless small globules which may exist in 
large numbers after an excessive milk diet or in the shape of small needle-shaped crystals, or again in the form of sheares. The small crystals of needle shape usually occur singly, and consist mostly of fatty acids, while the sheaves consist of fatty soaps. The fatty-acid crystals melt and disappear when heated, while the soaps remain unchanged. Ether likewise causes a disappearance of the fatty acids, while the soaps remain unchanged. Rieder' suggests the use of the dye stuff Sudan II. $\left(\mathrm{C}_{22} \mathrm{H}_{10} \mathrm{~N}_{3} \mathrm{O}\right)$ in a concentrated alcoholic solution for the differentiation of the fats. This dye stains plain fat bright red, while crystals of fatty acid and of lime and magnesia soaps remain unchanged. While normally these different forms of fat appear in very scanty amounts in the frees, they may be found considerably increased under pathological conditions (affections of the liver, pancreas, and acute enteritis).

Ciystals. - Besides the crystals of fatty acids and their soaps the following crystals are met with in the freces: oxalate of lime appears in the well-known envelope form of varying size, especially after a diet consisting principally of regetables. Calcium carbonate occasionally occurs in the form of amorphons granules or dumbbell-shaped crystals. Neutral phosphate of calcium and ammonio-magnesium phosphate crystals are often present and can be readily recognized, the former occurring in more or less well-defined wedge-shaped crystals collected into rosettes, the latter presenting the well-known coffin shape. They are soluble in acetic acid. All the crystals just mentioned are found in normal as well as in pathological freces, and have no diagnostic importance. Bismuth erystals: when bismuth is internally administered it is usually found in the fæces in

${ }^{1}$ Rieder: Deutsches Archiv für klin. Med., 1898, Bd. 59, Heft 3 and 4. p. 444. 
rhomboid crystals of a dark-brown or almost black color (Fig. 20). Hrmatoidin crystals are occasionally encountered in serere catarrhal conditions of the intestines or shortly after intestinal hemorrhages have taken place. They occur in small amorphous particles of an orange or

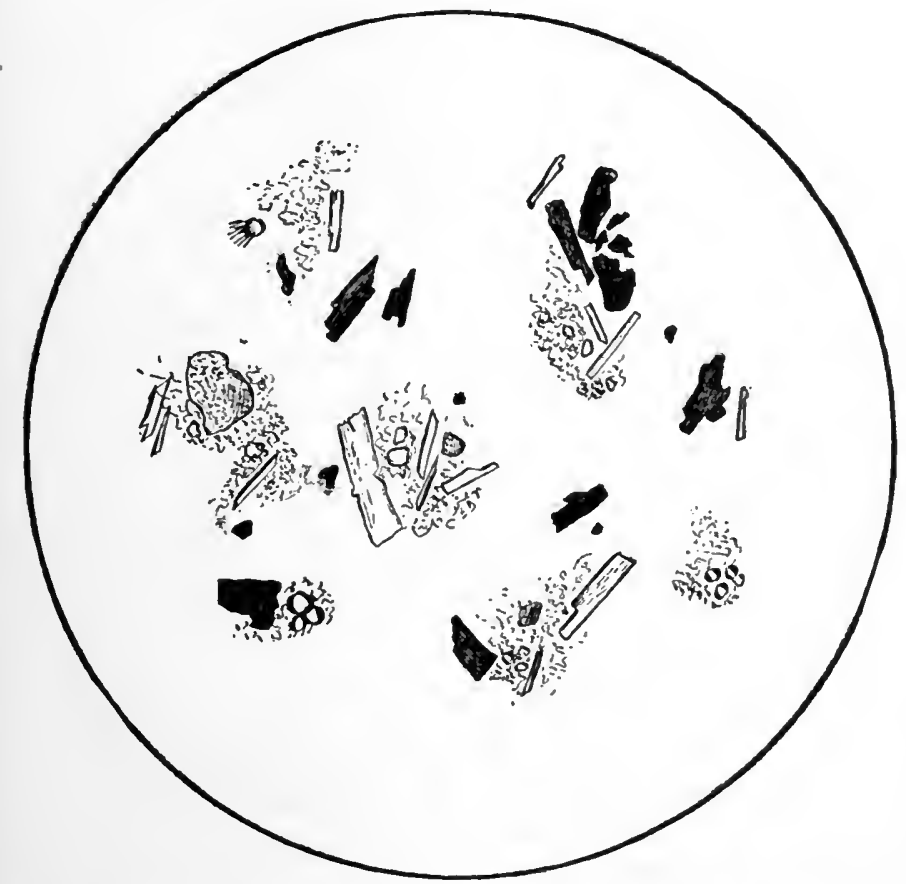

FIG. 20.- Specimen of Stool of Mrs, W., living on Milk Diet and taking Bismuth and Magnesla. Bismuth and magnesia crystals, some fat globules and detritus. No muscle or plant cells.

ruby red color, or in crystals of the rhombic system. Charcot-Leyden crystals of spermin phosphate, having the shape of grains of oats, are occasionally met with in the freces and are of diagnostic importance. According to Leichtenstern, ' these crystals are very frequently found in

'Leichtenstern: Deutsche med. Wochenschrift, 1892, No. 25. 
the frees whenever intestinal parasites (helminthiasis) exist. These crystals, however, occur also in other pathological conditions as in typhoid fever, dysentery, tuberculosis of the lungs. In rare instances the Charcot-Leyden crystals are absent in cases of helminthiasis. When they

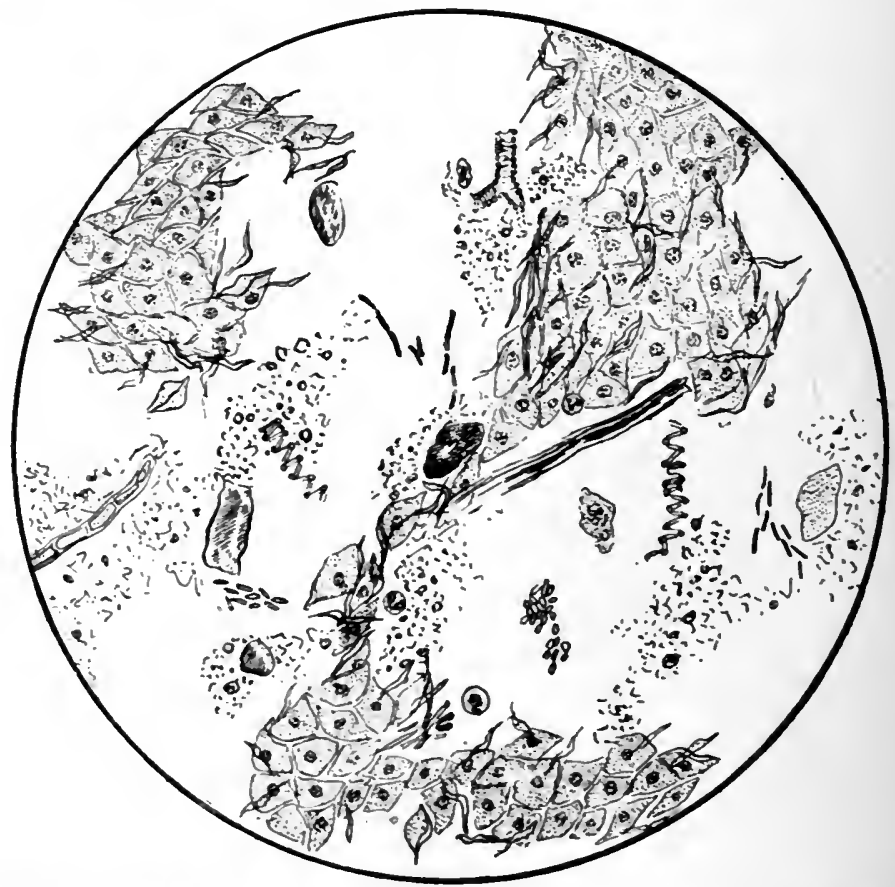

Fig. 21.-Specimen of Stool of Mrs. V., with (hronic Intestinal Catarrh. Groups of epitheltal cells ; detritus ; a lew muscle cells, partly digested ; plant cells; bacterla ; yeast cells.

occur, however, they are an indication that the stools should be carefully watched for the presence of intestinal worms.

Elements Derived from the Intestinal Wall.-Epithelial cells and also goblet cells occur occasionally in the freces, but only in scanty number (Fig. 21). They are very sel- 
dom unchanged with a distinctly risible nucleus; usually they appear in a metamorphosed condition without any perceptible nucleus. Larger accumulations of epithelial cells may be found in desquamative catarrhal conditions of the intestines.

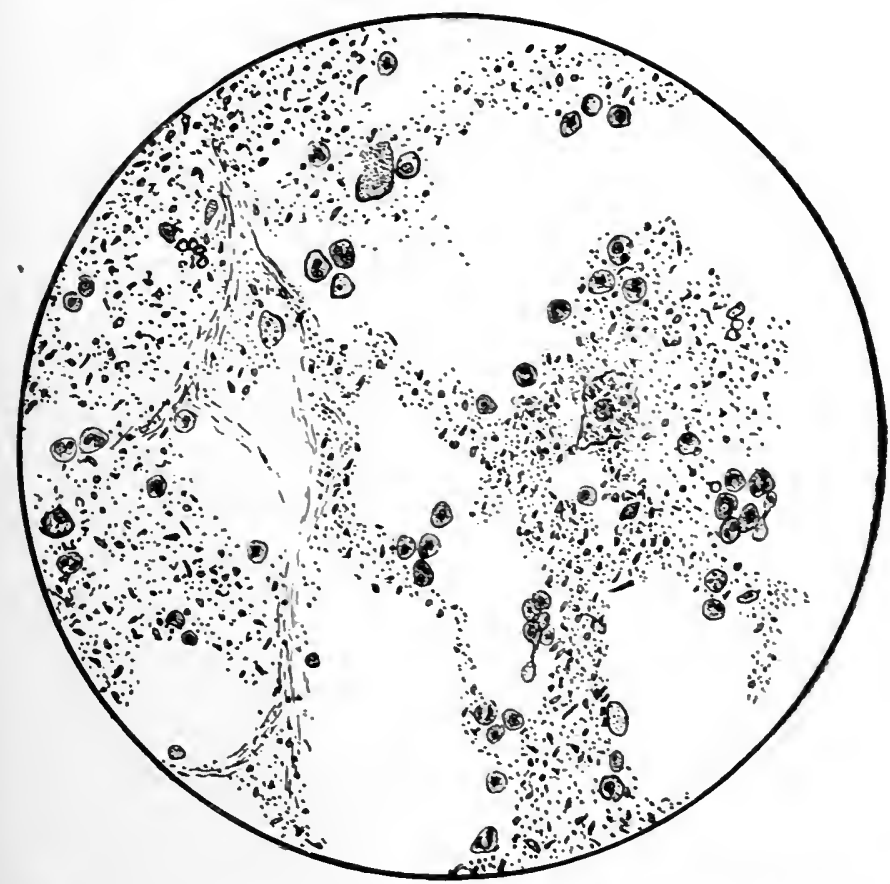

FIG. 2.-Stool of Patient L. with Acute Dssentery. Pus cells in considerable number: occasional epithelia : mucus ; detritus.

Blood.-Blood in the freces is occasionally easily recognized under the microscope, both red and white blood corpuscles being present. This, howerer, is the fact only in hemorrhages of the lower portion of the rectum. In hemorrhages originating in the upper portion of the large bowel or in the small intestine, the blood cells are usually 
already greatly changed and not to be recognized as such microscopically.

Pus.-Pus corpuscles in the dejecta occur in ulceratire processes of the intestines or whenerer an abscess has discharged its contents into the bowel. Besides these two conditions, it is also met with in drsentery. The pus

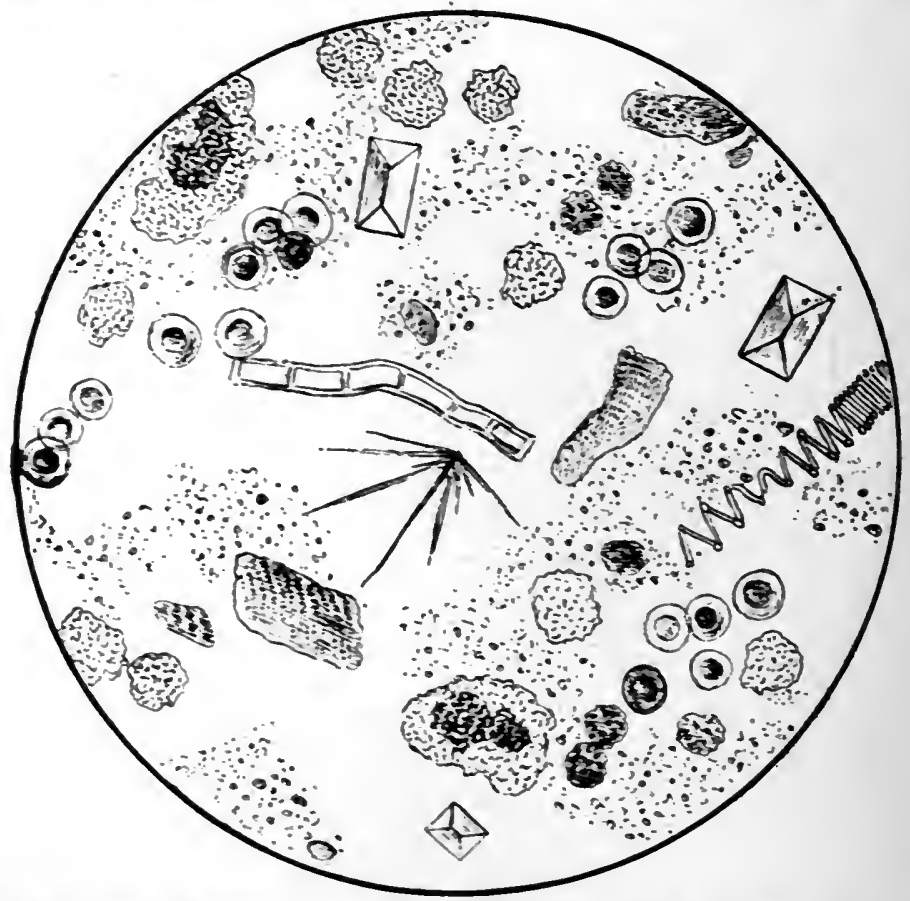

FIG. 3.-Stool of Patient H., with Chronic Dysentery, during an Acute Exacerbation. Highly magnifled. A moebre : red and wbite blood cells; crystals of fat and ammoniomagnesium pbosphate; plant and muscles cells; detritns.

corpuscles are then distinctly risible under the microscope (Figs. 22, 23, 24). [For the beautiful execution of the above drawings I am indebted to Dr. C. A. Elsberg of this city.] Mucus.-Mucus is frequently seen in the dejecta under the microscope. It is recognized by its thread-like ap- 
pearance (Fig. 25). Occasionally it is also amorphous. Thionin colors mucus reddish-riolet, while it stains other proteid substances blue. Mrucus is often present in catarrhal conditions of the intestine and also in membranous enteritis.

Pieces of Tumors. - In rare instances a small fragment of

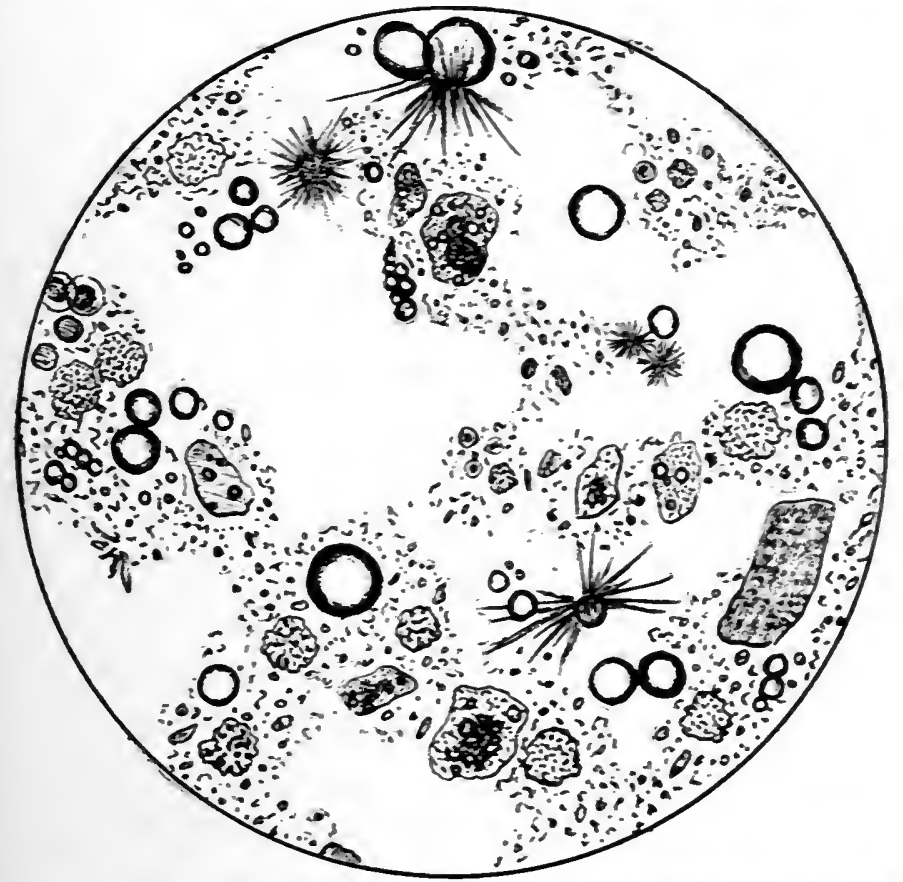

FIG. 24.-From the Same Patient, a Fer Dars Later. Highly magniffed. Amobre: fat in globules and crostals; a fer red and white blood corpuscles: muscles cells: detritus: hacteria.

tumor may be found in the dejecta. Under the microscope the structure of the mass will be seen and its character determined. The result of such an examination mas be of great diagnostic importance.

Nicro-arganisms. - Numerous micro-oryanisms are found 
in the fæces normally as well as pathologically. Their number averages in daily evacuations fifty-three milliards. Sometimes they may reach as high a figure as four hundred milliards. Beginning with the stomach the number of micro-organisms steadily increases all throngh the intestinal tract down to the large bowel, where the maximum is

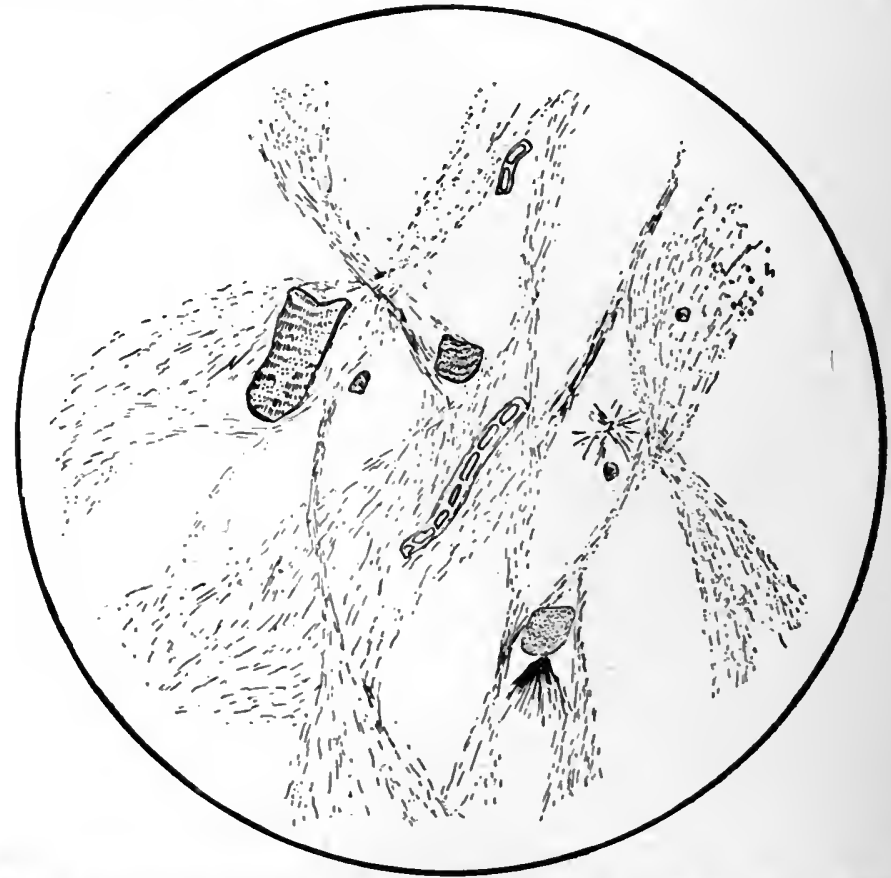

Fic. 25.-Specimen of the Stool of Mrs. J. B., Suffering from Intestinal Catarrh. Mucus all over the field of vision: a few plant cells and muscle cells, and an occasional fat erystal.

reached. The micro-organisms appear to be intimately connected with the physiological processes of digestion. This is true notwithstanding the valuable investigations of Nencki, Macfadyen, and Sieber, ' and Thierfelder and Nut-

'Nencki, Macfadyen, und Sieber: Archiv f. experimentelle Pathologie u. Pharmakologie, Bd. 28, S. 301. 
tal,' which have shown that normal digestion is possible even without bacteria. Pathologically various kinds of bacteria play a very important part. Besides certain species of pathogenic bacteria, the micro-organisms normally sojourning in the intestine occasionally assume morbific properties.

The different varieties of micro-orgauisms in the intestinal tract have been thoroughly studied by Maunaberg, ${ }^{2}$ who found fourteen different species of bacilli, nine species of micrococci, and four species of schizomycetes. Of the latter saccharomyces cerevisix are most frequently encountered in the fres. They are found in groups forming three or four buds, and assume a mahogany color when treated with Lugol's solution. Of the bacteria and cocci the following deserve special mention:

The bacterium coli commune, first described by Escherich, ${ }^{3}$ occurs in the form of thin or thick rods being about $0.4, \mu$ in length. Some show motile power. They are well stained by the ordinary anilin dyes and decolorized by Gram's solution. Their colonies growing upon gelatin resemble those of the bacillus of ty phoid fever.

The bacterium lactis aërogenes (Escherich) greatly resembles the bacterium coli commune. It is frequently found in the stools of infants, and is now and then met with in those of adults. It is found in thick rods frequently lying in pairs. They are non-motile aud have the property of causing fermentation of milk, producing coagulation and formation of gas within sixty hours.

1'Thierfelder u. Nuttal : Zeitschrift f. phys. Chemié, Bd. 21, S. 109, u. Bd. 22, S. 62 .

${ }^{2}$ Mannaberg : "Die Bacterien des Darms"-Nothnagel's Erkrankungen des Darms, Wien, 1895.

${ }^{3}$ Escherich : "Beiträge zur Kenntniss der Darmbacterien." Münchener med. Wochenschr., 1886, No i., 43-45. 
Bacillus putrificus coli (Bienstock ${ }^{1}$ ) forms slender rods $3 \mu$ in length. This bacillus energetically decomposes proteid substances in presence of air under the formation of ammonia, amin bases, fatty acids, tyrosin, phenol, indol.

While all the above-mentioned micro-organisms give a mahogany or brown color with solutions of iodine, there are a few varieties which give a blue color with this substance. To the latter belongs the bacillus butyricus described by Nothnagel. ${ }^{2}$ It is rod-shaped, 3 to $10 \mu$ long and $1 \mu$ thick. It is often lemon-shaped. This bacillus is anaërobic and produces fermentation of starch, sugar, and cellulose, forming butyric acid and gas. The bacillus butyricus is often found in pathological conditions of the intestine, but occurs in small numbers also in normal fres.

Of the pathogenic micro-organisms, cholera, ty phoid, and tubercle bacilli are found in the freces. The cholera and typhoid bacilli causing infectious diseases do not belong, strictly speaking, to the micro-organisms producing diseases of the intestine alone. The tubercle bacilli, occasionally producing intestinal tuberculosis, are recognized in the freces by the same methods which are employed in the examination of the sputum.

\section{TREATMENT.}

\section{Diet.}

The principles of diet are fully described in my book on the stomach. Here I will add a few remarks referring to the dietetic treatment of intestinal diseases. As in the. case of the stomach, acute intestinal disorders lasting a

'B. Bienstock: "Ueber die Bacterien der Faeces." Zeitschr. f. klin. Med., Bd. 8. 1884.

'H. Nothnagel : "Die normal in dem Menschendarm vorkommenden niedersten (ptlanzlichen) Organismen." Zeitschr. f. klin. Med., Bd. 3, 1881. 
few days or weeks must be managed according to the principle of rest. Very scanty and light foods (mostly liquid) should be given. In chronic ailments of the intestines the principle of rest may also be utilized occasionally for a short time, while as a general rule we should bear in mind the necessity of introdncing sufficient quantities of food and gradually accustoming the intestinal tract to the ordinary foods.

In some instances it is possible to exert a wholesome influence upon the disturbances of the intestine by appropriate dietetic measures. This applies especially to disorders accompanied by constipation or by diarrhoea.

I. Articles of diet which increase the intestimal peristalsis or "laxutive foods" are the following: Most fruits, both" raw and cooked, and fruit juices increase the peristalsis in consequence of the organic acids which they contain, as apples, pears, plums, peaches, strawberries, gooseberries, dates, and figs. Most salads and garden vegetables also increase peristalsis, firstly, owing to the large amount of water they contain, and secondly, owing to the considerable residue which is left undigested, as, for instance, melons, cucumbers, tomatoes, pumpkins, all kinds of cabbage. By many of the latter foods the peristaltic action of the intestine is also increased on account of the formation of acid and gaseons products. Fresh beer, cider, bonny-clabber, and kumyss act in a similar manner. Cold drinks of plain water or carbonated water act as mild aperients in some instances. Here a reflex action upon intestiual peristalsis due to irritation must be assumed, for often a movement of the bowels follows very soon (a quarter of an hour to one hour) after drinking.

II. Articles of diet which diminish the intestinal peristalsis or "constipating foods": (1) All substances con- 
taining a considerable portion of astringent agents, particularly tannic acid, as, for instance, dried bilberries, French red wines (particularly San Rafael wines), tea, cacao, the acorn preparations like acorn coffee, acorn cacao. (2) Foods which have a mucilaginous character and thus somewhat allay irritation also have a slightly constipating effect: sago, tapioca, barley, rice: (3) Foods which leave no residue whatever or very little residue, and thus exert no irritatiou. To these belong egg water (prepared by dissolving the white of an egg in some water), scraped raw meat, mutton broth.

Some foods manifest different action in different individuals. Thus, for instance, milk is constipating in one per"son and laxative in another, while in still others it has no special effect upon intestiual peristalsis.

Most foods have no marked influence upon the intestinal peristalsis. To these belong most kinds of meat and fish not too highly seasoned, the various meat powders, and most artificial foods like meat peptone and nutrose, eucasin, somatose, sanose, eggs prepared in different ways, wellbaked bread, wheaten or rye bread, crackers, zwieback, fats in small amounts, especially butter. The preparation of the foods has an important bearing with regard to its action upon the intestinal peristalsis. The finer the foods are the less irritating they will act, and the coarser the particles the greater the irritation they produce upon the intestinal muscular layer. Highly seasoned foods also act as a stimulant of the peristalsis.

In some severe conditions of the intestines the ordinary way of ingestion of food must be avoided for a short period. Here artificial feeding is employed. Artificial feeding can be done in two different ways: rectal alimentation and subcutaneous alimentation. 
1. Rectal Alimentation.-The rectum and the greater part of the large bowel should be emptied if possible before injecting the feeding enema. The latter is best accomplished by using a fountain syringe and a soft-rubber tube which is introduced for about five to seven inches into the rectum. The quantity of the feeding enema may be between five and ten ounces. As feeding enemas the following substances are used: ( $($ ) The different kinds of peptones and propeptones in the market of which about two to three ounces can be dissolved in six to eight ounces of water. The different beef juices may also be dissolved in water and injected in corresponding quantities. (b) The milk and egg enemas. These are mostly used. Their composition is as follows: Six to seven ounces of milk, one or two raw eggs well beaten up, one teaspoonful of powdered sugar, and the point of a kuifeful of salt. The addition of pancreatin (one tube of Fairchild pancreatin to one enema) will facilitate assimilation. (c) Meat-pancreas enema. Leube 'employs enemas consisting of wellchopped meat mixed with fresh pancreas.

Besides these food enemas injections of water. into the bowel are made in order to increase the amount of fluid in the system. These injections of water for the purpose of absorption are of great importance. Usually saline solutions are employed in quantities varying from one pint to one quart. The nutritive enema should be given three or four times in twenty-four hours, and the water enemas for absorption once or twice a day.

2. Subcutaneous Alimentation.-In diseases of the intestine special conditions are met with in which neither the ordinary way of feeding nor rectal alimentation is possible.

${ }^{1}$ Leube : Leyden's “Handbuch der Ernährungstherapie," Bd. i., p. 508, Leipsic, 1897. 
Here an attempt must be made to introduce nourishment subcutaneously. Most food substances cannot be introduced under the skin without inflicting more or less injury. Two substances only form an exception and are of practical ralue: (a) Olive oil. This can be injected subcutaneously to the amount of one ounce twice or three times a day. It is hardly necessary to say that the oil as well as the syringe used for this purpose should be thoroughly sterilized. A large-sized Pravaz syringe is employed, and but little pressure exerted while injecting. This precaution is necessary in order to obviate any traumatism (tearing) of the tissues. The best place for the injection is the thigh. Water. A saline solution is subcutaneously injected in amounts varying from one pint to a quart. This serves to increase the amount of fluid in the system. The injection is made by means of the fountain syringe to the end of which an aspirating needle is attached. The same precautions as above are necessary. The saline injection may be employed twice or three times a day if necessary.

\section{Mechanical Procedures.}

Injections.-Injections into the bowel in the form of clysters were used for curative purposes eren in old times. The regular syringe with its stiff end may, if forcibly inserted, give rise to damage of the rectum. For this reason nowadays a soft-rubber rectal tube is employed, to which a fountain or Daridson syringe or any form of syringe can be attached. The tube being flexible cannot injure the intestinal walls. It can also be introduced higher up than the ordinary hard-rubber end pieces of the fountain syringe. Instead of the fountain syringe a funnel apparatus similar to the one used in gastric larage may be employed. For washing out the bowel Leube-Rosenthal's appliance for 
washing out the stomach can be used to adrantage. For irrigation of the bowel Kemp's hard-rubber rectal doublecurrent irrigator can be conveniently employed (Fig. 26).

These injections into the bowel are made for various purposes:

1. To produce an evacuation. About a quart of lukewarm water to which a teaspoonful of salt is added can be employed, or a piece of soap dissolved in the same

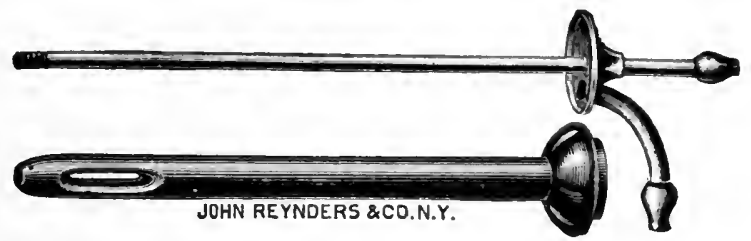

FIG. 26.-Dr. R. C. Kemp's Rectal Irrigator (New Model). Outer tube of bard-rubber: central tube of metal. Hard-rubber flange, protecting sphincter from transmisslon of heat through the metal parts.

amount of water. As a rule, it is not advisable to introduce larger quantities of water than these as they distend the bowel too much. In greatly atonic conditions, however, in which a quart of water may be ineflicient, an injection of from two to three quarts will be required. Injections of oil (olive oil or sesame oil) in quantities varying from half a pint to one pint have been recommended by Fleiner.' According to this writer the oil should be injected at blood temperature into the rectum when retiring and be retained over night. While olive oil was used as a laxative injection long ago by Habershon ${ }^{2}$ and others, we owe its methodical use to Fleiner, to whom is also due the credit for having promulgated the method. Small injections of glycerin (one or two drachms) in about an ounce

${ }^{1}$ Fleiner: "Ueber die Behandlung der Constipation." Berl. klin. Wochenschr., 1893, Nos. 3 and 4.

${ }^{2}$ Habershon: "Diseases of the Abdomen," London, 1862. 
of water can also be advantageously employed for producing an evacuation of the bowels.

2. Injections may be resorted to either to strengthen the tonicity of the bowel, in which case plain very cold water in amounts of from one to two quarts can be employed, or for medicinal purposes, i.e., for applying certain medicaments directly to the intestinal mucosa. The drugs most frequently used for this purpose are nitrate of silver, tanuic acid, subnitrate of bismuth, as astringents; thymol, hydrogen peroxide, boracic acid, essence of peppermint, as disinfectants.

Massage and Gymmastic Exercises.-Massagre is frequently employed in functional diseases of the intestine. Its field of usefuluess lies principally in neurotic and atonic conditious. Massage should be applied by well-trained and experienced persons. Abdominal massage requires great care, as too rough manipulation is liable to do great harm. Gymnastic exercises and sports are well adapted to stimulate and strengthen the muscles of the abdomen as well as those of the intestine. Ewald particularly recommends rowing in boats with sliding seats as an exercise which gives definite results in chronic intestinal torpidity. Golf, billiards, horseback riding, bicycle riding, walking may also be included among the exercises contributing to a toning up of the system.

Hydrotherapy. - Moist applications in the form of either Priessnitz's compresses or poultices are often of benefit. Priessnitz's compresses are stimulating, while the warm fomentations serve as a sedative. The latter are applied to allay pain, the heat producing a temporary paralysis of the superficial sensory nerves. Instead of either cold or warm compresses a rubber bag filled with either cold or hot water may be applied. When warm applications are 
required they can also be used in the form of the Japanese box. Sitz baths of various temperatures may be employed. A shower bath, especially over the abdomen, of cold or warm water or of alternating cold and warm water, is also of benefit. Many of these procedures may be combined with massage, and in this way the curative action is enhanced.

Electricity.-The faradic, galvanic, or franklinic currents are employed. All these three can be used percutaneously; the first two also intrarectally. The faradic current is mostly applied in atonic conditions of the bowel with the object of stimulating the motor function of the intestines. The galvanic

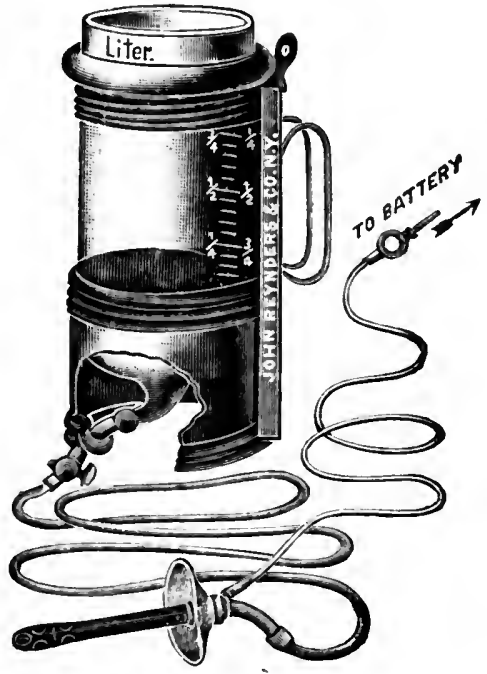

F1G. 2\%.-Rectal Electrode. current is principally employed in painful intestinal affections of neurotic character. The franklinic or static current may be advantageously used in both conditions. For the intrarectal application of the current I use an electrode which in principle is very similar to that of Boudet ${ }^{1}$ and consists of a perforated hard-rubber end piece in which is lodged a metallic button connected by means of a wire with the battery. To the upper end of the hard-rubber piece is attached a soft-rubber tube leading to an irrigator and pro-

'Boudet: Cited after A. Mathieu: "Treatment of Diseases of the Stomach and Intestines," New York, 1894, p. 171. 
vided with a stopcock (see Fig. 27). Proceed as follows: The irrigator is filled with water at blood temperature. The hard-rubber piece, or the rectal electrode, is smeared with raseline and introduced into the rectum. Another plate electrode is moistened and placed over the abdomen, the stopcock partly opened, and the current applied. The water running from the end piece of the electrode into the bowel carries the electricity along with it. The electrical application should last from five to ten minutes, the amount of water used varies from ten to fifteen ounces. The outflow of the water can be regulated by the stopcock arrangement. I have applied both the faradic and galranic currents with this apparatus and found it very conrenient. The faradic current may be applied as strong as the patient can bear, while the galvanic current should be used with the negative pole in the rectum, the intensity of current ranging from eight to fifteen milliamperes. 


\section{CHAPTER III.}

\section{ACUTE AND CHRONIC INTESTINAL CATARRH.}

\section{ACUTE INTESTINAL CATARRH.}

Symomyms: Euteritis acuta; Catarrhus intestinalis acutus; Acute diarrhoea; Cholera nostras.

Definition.-An inflammatory affection of the intestines characterized by a sudden development of pains and more or less loose morements.

Etiology.-Acute intestinal catarrh is one of the most frequent diseases. While it oceurs more often in infants and children it is found in persons of all ages.

The affection may attack the entire intestinal tract or may be limited to a part of it. Thus we mas hare a duodenitis, jejunitis, ileitis, trphlitis, colitis, and proctitis (inflammation of the rectum). With regard to frequency the colon is most often affected. According to Woodward, ${ }^{2}$ an inflammation of the small intestine alone hardly erer exists, a portion of the large borel alwars being affected. Intestinal catarrh is either primary (idiopathic) or secondary when occurring as a sequel of other diseases. Acute enteritis may be due to a number of causes:

1. It may result from the ingestion of heary indigestible food, ice-cold drinks, and tainted meat or fish, unripe fruit, stale or sour beer, bad water.

2. Good food and drink taken in unusually large quantities may also produce this condition.

1 Woodward: "The Medical and Surgical History of the War of the Rebellion," vol. i , part 2. 
3. A host of organic and inorganic substances may chemically irritate the intestinal mucosa and cause inflammation. All the drastic remedies, like croton oil, colocynth, jalap, etc., belong to these organic irritating substances; of the inorganic may be mentioned tartar emetic, arsenic, lead, sulphate of copper, all the mercurial preparations, concentrated acids, and stroug, caustic alkalies.

4. Enteritis may be caused by mechanical irritants. Thus hardened scybala, biliary calculi, enteroliths, or foreigu bodies which have been swallowed, like large kernels of fruit or coins, may evoke inflammation. The catarrh accompanying intestinal worms may also be placed in this group.

5. Intestinal catarrh is very often due to variations in temperature or to catching cold. It seems that the disposition to this agent varies in different individuals. Thus some people get an attack of diarrhoea if they sleep uncovered during the summer and a drop in temperature occurs, the colder atmosphere affecting the abdomen. Others, again, are attacked with diarrhoea whenever they get their feet wet. How the inflnence of cold acts in causing the enteritis is difficult to say. Some writers believe that the surden change in the circulation of the blood caused by the cold is the principal factor; others again explain it on the ground of a more favorable development of microorganisms during the cliange of temperature.

6. Auto-intoxication. Poisonous substances may develop in the intestinal tract and cause diarrhoa. The enteritis following large burns of the skin belongs to this group. Here the poisonous substance is probably formed at the site of the burned skin and carried by the blood current into the intestinal tract.

Secondary catarrh of the intestine occurs in almost all 
acute infectious diseases in the same way as gastric catarrl. It is further found accompanying heart, kidney, and liver diseases, tuberculosis, diabetes, etc. Most organic diseases of the bowels are associated with intestiual catarn, as cancer of the intestines, volvulus, invagination, peritonitis, thrombosis. In this class of cases, however, the intestinal. catarrh is of little importance compared with the primary affection.

Morbid Anatomy. - The anatomical changes found in autopsies are not always very well marked, and there is cer.. tainly no exact relation between the iutensity of the clinical symptoms and the severity of the pathological processes discovered. The mucous membrane of the affected part of the intestine appear's reddened either over its entire extent or only in spots. This red color is more pronounced around the follicles and patches, at the apex of the folds and of the villi. If the process is intense, extravasations of blood may be found. The mucous membrane appears swollen, sometimes oedematous, often it is corered with tenacious mucus. The villi and the solitary follicles are succulent and appear as whitish, small prominences surrounded by a red stratum (enteritis follicularis seu nodularis). If the process continues, these gray areas may rupture, and thus give rise to ulcerative lesions (follicular ulcers). Catarrhal ulcers also exist, however, caused by the loss in some places of the protective epithelial covering of the mucosa. Through extension of the inflammation in width and depth irregular losses of substance with undermined edges are produced. Inflammatory irritation in the neighborhood of these defects may give rise to polypoid growths, especially when the process has run a protracted course.

Microscopically the vessels of the mucosa and sub- 
mucosa appear in a more or less congested state. Small extravasations often exist between the glands of Lieberkuehn. The spaces between the glands are frequently widened and filled with au abundant accumulation of round cells. The epithelium of the mucosa has mostly disappeared, especially in the large bowel. But according to Nothuagel this may be a post-mortem phenomenon and not always the result of inflammation. Desquamative processes in the epithelial layer, howerer, occur during life caused by the catarrhal affection, for the changed eroded epithelial cells are found in the mucus voided with the stool. The glauds often appear altered with regard to their contour, being wider at their fundus and much narrower at their month, frequently presenting a flask shape. The submucous tissue is usually somewhat hyperplastic, otherwise not much changed. The muscular and serous coats are not affected.

Symptomatology.-Intestinal catarrh usually manifests itself through a feeling of fulness in the lower part of the abdomen, colicky pains appearing from time to time, and diarrhoea. As a rule, no fever is present except in cases of a serere type. The number of the stools and their quality vary a great deal. In mild cases there may be only two or three movements in twenty-four hours; in sererer cases fifteen to twenty diarrhoal evacuations. The first passage as a rule still contains normal fecal matter in its first portion, while the second part is of a mushy character. The next morements are semi-fluid, and at last entirely liquid dejecta may appear. The first stool still has a brown color and the characteristic fecal odor, while the following evacuations present a slightly yellowish color or even a grayish appearance, occasionally resembling rice-water. The latter are sometimes devoid of fecal odor, have an acid reaction, 
and show a foamy surface. Mucus is almost always present. The fecal matter in its yellow parts contains, as a rule, unchanged biliary substances which give a characteristic Gmelin reaction. Microscopically undigested food particles may be discovered in larger than normal amounts; thus meat fibres and well-preserved granules of starch may be observed. A host of micro-organisms, epithelial cells, sometimes in contiguous groups, and mucus are found. Very seldom and only in severer cases small amounts of pus and red blood corpuscles may be discovered. Chemically peptones and sugar may be found in the dejecta.

General Subjective Symptoms.-Aside from the diarrhceal movements and the unpleasant sensations consisting in a feeling of pressure and fulness in the abdomen mentioned above, there may in light cases be perfect euphoria; usually a feeling of weakness exists which is especially marked in the lower extremities. A feeling of clizziness and slight nausea often also appear, especially shortly before and during evacuations. Vomiting may also occur, as a rule, in cases in which the stomach is likewise affected or when the process of inflammation is of a severer type (cholera nostras). Tenesmus is frequently present, if the process is in the lower part of the colon, even if not especially pronounced. This seems to be the result of the irritating action of the dejecta upon the rectum.

The general symptoms above described are much more pronounced in children and very old people. Here the appearance of collapse (cold extremities, blue lips, and apathy) is not very rare. Marshall Hall ${ }^{1}$ has described a condition under the name of acute hydrocephaloid disease

${ }^{1}$ Marshall Hall : "Diseases and Derangements of the Nervous System," London, 1841, p. 153. 
which occurs in weak children with acute enteritis. The hy drocephaloid appears in consequence of serere attacks of gastro-enteritis with a temperature of $104^{\circ}-106^{\circ} \mathrm{F}$. There is sudden collapse. While the body is hot, the extremities become ice cold, the fontanelles sink in, the pulse becomes considerably accelerated, soft, and often irregular. In this condition the little patient lies apathetic unless suddenly disturbed with colicky pains when he utters a cry. The pupils do not react alike and the conjunctival reflex may be absent. Sometimes paralysis of the rectum is present, which I have seen in one case. In this condition the patient often dies within a short time from paralysis of the heart.

Objective Symptoms. - The physical examination of the abdomen occasionally reveals on inspection a bloated condition and some spots tender to pressure. As a rule, the lower part of the abdomen, particularly the immediate neighborhood of the navel, is slightly painful on palpation. Occasionally there may be found a decided tenderness, either in the right or in the left iliac region. Sometimes this tenderuess may be quite pronounced in a line runuing across the abdomen between the margins of the false ribs (transverse colon). Palpation often elicits gurgling sounds caused by intestinal coils distended with gas and fluid contents. This phenomenon is most frequently observed in both iliac regions.

In patients with thin abdominal walls peristaltic movements of the small intestines may be visible either spontaneously or after palpatory examination.

The urine is voided in small quantities, is concentrated, and often shows Rosenbach's reaction (Burgundy red color after boiling with nitric acid), and also contains indican (this especially if the process involves the small intestine). 
Casts and small amounts of albumin are sometimes found in the urine, especially in severer cases (Fischl ').

Fever.-In the greater number of instances there is no rise of temperature during the course of this affection. In some cases, howerer, ferer is quite a prominent symptom, and the disease may commence with violent chills and a marked elevation of temperature $\left(104^{\circ}\right)$. The temperature may either fall suddenly on the next day or after the lapse of a few days, but it does not show that regular steady rise which is characteristic of typhoid fever. Fever is especially met with in those cases of acute enteritis which are caused most probably by infection (either pathogenic microorganisms or tainted food).

Locatization of the Catarrhal Process.-In order to find out what part of the bowels is especially affected the following points are of value:

A duodenitis may be recognized if the above symptoms are accompanied by icterus. Intestinal catarrh attended with a constant painful sensation in the right epigastric region, which, besides, is also tender to pressure, indicates more or less a continuation of the catarhal process from the stomach to the duodenuy. Pains appearing in the same region after extensive burns of the skin also point to a duodenal affection, even if there be no icterus.

Jejunitis alone or jejunitis and ileitis without any affection of the large bowel can be diagnosed only with difficulty, for the principal sy mptom of enteritis (namely, that of diarrhœa) is as a rule absent. Small amounts of mucus well mixed with fecal matter, a considerable quantity of undigested food particles, and epithelial cells tinged with yellow bile pigment in the freces, point to a catarrhal condition of the small intestine. Indicanuria is also often present.

${ }^{1}$ Fischl : Prager Vierteljahresschr., 1878, Bd. 139, p. 27. 
Acute colitis is claracterized by painful sensations and a greater tenderness on pressure over the entire colon. The stools are diarrhoeal and contain large quantities of mucus. The latter as well as the fecal matter may contain undecomposed biliary pigment. Sigmoiditis, described by Mayor ${ }^{1}$ and later by Boas ${ }^{2}$ and Mathews, ${ }^{3}$ means an inflammatory process involving the sigmoid flexure, and is recognized by special tenderness on palpation of this portion of the bowel, intense backache, and a frequent disposition to go to stool.

Proctitis, or inflammation of the rectum, is characterized by severe tenesmus and colicky pains in the left iliac fossa. The patients have a constant desire to go to the closet, but at each time void only small quantities of fecal matter under the greatest pains. The scybala are surrounded by a layer of nucus which may be tinged with blood. Occasionally the mucous membrane of the rectum prolapses during defecation. It then appears intensely dark red and is extremely painful to the touch. Even if not prolapsed, a digital rectal examination is attended with much pain. The inucous membrane of the rectum feels hot and the examining finger on removal sometimes shows traces of blood.

Duration. - The duration of acute enteritis varies considerably. Mild cases improve in about two to five days, while those of a severer type may last about two weeks. After recovery from acute enteritis the intestinal tract remains quite sensitive for a long time. If no attention is paid to this condition and gross errors of diet are committed, relapses are liable to occur. Several relapses may

'A. Mayor: Revue méd. de la Suisse Romande, 1893, No. 4.

'J. Boas: "Krankheiten des Darms," ii., p. 513.

"Mathews: "Disease in the Sigmoid Flexure." The American Medical Quarterly, June, 1899. 
also follow each other and ultimately cause a chronic enteritis.

Diagnosis.-As a rule the recognition of acute enteritis is rery easy. The characteristic diarrboa, the admixture of mucus in the dejecta, the fact that a dietetic error has been committed, or that the abdomen (or other parts of the body) has been exposed to cold, will all indicate the nature of the affection. The localization of the process, whether affecting more or less the entire intestinal tract or only certain parts, is more difficult, and the important points of differentiation have already been given above. Frequent romiting and very pronounced general symptoms (especially collapse) point to cholera nostras, which is the most severe form of acute enteritis. If the diarrhoea is accompanied by high fever, urinary casts, and pains in the muscles and joints, then the assumption of an acute enteritis of an infectious type is justified.

Prognosis.-The prognosis of acute enteritis is, as a rule, good, the disease tending to recovery in a very short time. In children, however, and very old and weakened persons, the course of the disease is sometimes not so farorable and may lead to collapse and even to deatl.

Treatment.-In mild cases of acute enteritis no medicinal treatment will be necessary. Abstinence from food for one or two days, allowing the patient to take only weak tea, a small quantity of bouillon, and some boiled water may suffice to check the attack. Sometimes, however, especially if the attack of enteritis has been caused by dietetic errors, and fulness of the abdomen and frequent colicky pains indicating that irritating substances are lodged within the intestines are present, a good old-fashioned drastic is in place. Thus castor oil-abont one ounce-may be given or calomel 0.6 (gr. x.), the latter being preferable in cases 
of a probably infectious nature. If there is no fever and the sy mptoms are mild, then the patients may be up and about, although it is always advisable for them to keep quiet more or less. In cases of a severer type, and especially those with fever, the patients should stay in bed until the symptoms are entirely subdued. If the diarrhœa shows no signs of abating after a day or two, or if the symptoms occur so frequently as to be debilitating, then an opiate is in place. Tincture of opium, seren drops every three hours, or codeine, 0.02 or 0.03 (gr. $\frac{1}{3}-\frac{1}{2}$ ) also every three hours, may be given. Frequently the combination of an opiate with subuitrate of bismuth and chalk or with tannigen may be useful. Thus I often prescribe the following powders:

R, Bism. subnitr ................... 6.0 ( 3 iss.)

Cret. pulv ....................... 3.0 (gr. xlv.)

Cod. phosph .................... 0.1 (gr. iss.)

Elwosacch. menth. pip ............... 5.0 (gr. Jxxv.)

Nisce f. pulv. Div, in p. æq. No. x. S. One powder three or four times a day.

Or-

B Morph. muriat ................. 0.1 (gr. iss.)

Tannigen,

Elæosacch. menth. pip............... āā 5.0 (gr. lхxष.)

Misce f. p. Div. in p. æq. No. $x$. S. One powder three times daily.

Calumba, cascarilla, catechu, kino, may also be employed, twenty to thirty drops of the tiuctures being given about three times daily. Another useful remedy is dermatol, which may be administered in doses of $0.5 \mathrm{gm}$. (gr. viii.) three times daily. In cases in which the entire colon or its lower part is affected, irrigation of the bowels with astringent solutions is of great benefit. This may be done with a solution containing nitrate of silver, 0.3 (gr. v.) 
to 1,000 (one quart) water, or tannic acid, 2 to $5 \mathrm{gm}$. (30 to 80 grains) to 1,000 water, or liquor ferri sesquichlor. $2: 1,000$. It is best to inject these solutions after a previous washing out of the. bowel with plain water or soon after a morement. The astringent solution should be allowed to remain for about five to ten minutes, but in case the patient is not able to retain it for even so short a time, fifteen to twenty drops of tincture of opium may be added to the injection. This, as a rule, lessens the irritation of the rectum and the patient is thus able to hold the enema longer. The temperature of the water should be tepid. All the above-mentioned astringent remedies have also slight antiseptic qualities. In cases, however, in which the fermentative processes within the bowels are especially pronounced, the following stronger antifermentative substances may be used for irrigation: salicylic acid, $2: 1,000$ water, or salicylate of sodium, 10.0 ( 3 iiss.) : 1,000; boracic acid, 5.0 : 1,000; creolin, 1.0 (gr. xv.) : 1,000.

If pains are present a warm poultice or a hot-water bag over the abdomen is very beneficial.

Cold drinks should be forbidden. Warm teas, fennel or camomile, are useful; on the second or third day the patient can be nourished with soups or gruels (barley, rice, oatmeal soup cooked with or without milk); water soup (stale bread softened in hot water with the addition of a little butter and salt) and hot spiced claret are then in place. A little later toasted bread, crackers, soft-boiled eggs may be added to the diet; still later, scraped meat, lamb chops, tenderloin steak, bread and butter. As soon as the diarrhoea has entirely stopped we may allow mashed or baked potatoes in addition to the other articles. For quite a while after an attack of enteritis the patient has to 
be careful with vegretables and especially fruits. The first he may begin to take in small portions soon after an attack, while the latter should be aroided for a somewhat longer time.

In secondary enteritis the priucipal primary affection unust be considered first. Thus enteritis accompanying malaria will be best remedied by quinine. Enteritis accompanying affections of the lung, heart, or liver must be treated after due attention has been given to the primary affection.

\section{CHRONIC INTESTINAL CATARRH.}

Symomyms.-Enteritis chronica; Chronic catarrh of the bowels.

Definition.-An affection characterized by a chronic inflammation of the intestinal mucosa, giving rise to various disturbances in the function of the bowels.

Etiology.-Chronic intestinal catarrh may arise either from a severe acute enteritis which shows no tendency to a cure, or (most often) from repeated attacks of acute enteritis following each other at short intervals before the bowels have had a chance to recover fully. This often occurs in patients who do not pay sufficient attention to their apparently slight trouble and disregard the dietetic rules prescribed by the physician. The direct factors causing chronic enteritis are the same as those of the acute condition. Like acute enteritis, chronic intestinal catarrh may be divided into a primary and a secondary form, the primary being idiopathic, while the secondary appears in connection with affections predisposing to this condition. Thus diseases of the lungs, especially tuberculosis, affections of the heart, liver, and kidneys, and diabetes are often accompanied by chronic intestinal catarrh. Intestinal 
parasites, round worms, tapeworms, etc., are quite often the cause of a secondary chronic enteritis, due to the irritation of the intestinal mucosa which they evoke.

Morbid Anatomy.-The anatomical changes in chronic intestinal catar'h are similar to those of the acute condition and are characterized by hyperæmia, swelling, and increased secretion of the mucous membrane. However, instead of the bright red or intensely dark red color seen in acute catarrh, the mucosa in the chronic form presents a gray ish brown-red tint. The blood-ressels are greatly distended, and often curved into a serpentine shape. In cases of long duration the intestinal mucosa frequently appears of a slate color intermingled with black pigment (changed red blood pigment which has escaped from the blood-ressels). These black dots are often found accumulated at the tips of the villi and also in the immediate neighborhood of the lymph follicles and of the glands of Lieberkuehn. The surface of the mucosa is as a rule covered with a viscid and transparent mucus. The epithelial cells are cloudy, in a condition of fatty degeneration, and partly desquamated. The interstitial tissue is infiltrated with cellular elements. The glands themselves are of irregular shape, sometimes elongated and tortuous, occasionally much smaller than normally. In cases in which there is an interstitial tissue proliferation, a constriction around the neck of a gland arises. As a consequence there is retention of the glandular secretion, and ultimately a cyst may develop. Hy perplastic processes around the inflamed area very often lead to the formation of polypi. The latter as a rule consist of muscular and fibrous tissues and contain no glands. Exceptionally polypoid excrescences may appear on the intestinal mucosa (especially in the colon), which consist of a real proliferation of the intestinal mu- 
cosa containing glands. An excellent instance of this rare occurrence has been described by Woodward.'

In some of the most advanced cases, atrophy of the mucosa may be present. As in the stomach, this process may arise from two entirely different conditions. In the one the process originates in the glandular tissue; the latter becoming inflamed, the seat of fatty degeneration, and ultimately atrophied. In the second group the process leading to atrophy origiuates from an interstitial tissue proliferation; the connective tissue becoming hypertrophied, compresses the glands, and, gaining the upper hand, ultimately leads to their entire disappearance. These atrophic processes, as a rule, do not extend over the entire intestine, but more often involve certain parts. Thus, the cxecum and its immediate neighborhood have often been found in this state, even in persons who apparently during life had no intestinal affection (Nothnagel). Large portions of the small and large intestines or the entire intestinal tract are but rarely found atroplied, more often in children than in grown-up persons. Ewald ${ }^{2}$ mentious that he has observed this rare condition in six autopsies in adults. They all had suffered during life from pernicious anæmia and gastro-intestinal disturbances.

Both the hyperplastic and atrophic processes, as a rule, are not limited to the intestinal mucosa alone, but also involve the neighboring structures (the submucosa and the muscularis). Thus in the hyperplastic form the thickness of the wall of the small intestine may be increased to six times its normal size, while the large bowel may become

1 Woodward : L. c.

${ }^{2}$ C. A. Ewald: "Diseases of the Intestines." Twentieth Century Practice of Medicine, vol. ix., p. 127. 
three times as thick as normally. In atrophy of the intestine there is also a degeneration of the muscles. The ganglionic cells of the Meissuer and Auerbach plexus have been found in a state of fatty degeneration, smaller and lessened in number in the atrophic form (Jürgens' and Sasaki ${ }^{2}$ ). Whether these changes in the nerrous tissue are the canse or the result of this general intestinal atrophy is as yet not known.

Several varieties of ulcerative processes exist complicating chronic intestinal catarrh. Some ulcerations arise in consequence of superficial erosions of the mucosa, which do not heal. The defect, once produced, gradually grows deeper. Several superficial ulcers adjacent to each other may grow larger and unite. Thus a considerable irregular ulceration develops. The ulcerative process increasing in depth may lead to a secondary phlegmonous inflammation of the submucosa, and ultimately to perforation of the intestinal walls. Another danger lies in the ulcerative process involving a blood-ressel which may cause hemorrhage. If the perforation through the intestinal walls occurs rapidly, fatal peritonitis results; but if the perforative process develops slowly, then agglutination takes place and a localized peritonitis with or without the formation of a fecal abscess follows. These eventualities are; howerer, rare. Generally the ulcerations either remain unchanged (not progressing) for a long period of time or they cicatrize. In the latter event strictures of the intestinal lumen may oceasionally develop.

Follicular enteritis is also occasionally the cause of the formation of an ulcer. The lymph nodules swell up to pea size, soften, and burst. A small ulcer thus arises. As a

${ }^{1} J u ̈ r g e n s:$ Berl. klin. Wochensch., 1892, p. 357.

${ }^{2}$ Sasaki : Virch. Arch., Bd. 96, p. 287. 
rule, healing takes place, the mucosa of the immediate neighborhood extending over and gradually overlapping the defect. Sometimes, howerer, the ulcerative area is covered with a laser of mucus secreted by the goblet cells of the neighboring glands. From time to time the accumulated mucus is removed from the defect and appears in the dejecta in form of particles resembling sago. Extensire ulcerations are seldom met with in chronic enteritis. Most often they occur in the enteritis accompanying pulmonary tuberculosis.

Symptomatology.-Chronic intestinal catarrh may occasionally exist without giving rise to any subjective complaints. As a rule, howerer, there is a feeling of discomfort and sometimes of slight pains in the abdomen. These abuormal sensations may be especially marked some time after the ingestion of food or shortly before the evacuations. In some cases, again, these annoring sensations appear early in the morning, about an hour or two before rising. Borborrgmi often occur; occasionally there is a feeling of tension or of bloating in the abdomen, which may be relieved by the passing of flatus. The latter symptom may be so constant and annoring that the patient is afraid to appear in society or may be hindered in his vocation. An accumulation of gases in the intestine, especially in the colon, may sometimes exert pressure upon the diaphragm and give rise to asthmatic complaints, palpitations of the heart and angina pectoris, congestion of the head and rertigo. Belching or passing of wind alleviates these symptoms or entirely removes them.

Colicky pains sometimes appear and are of short duration. Severe pains, howerer, are almost always absent.

If the catarrh has lasted for some time, then symptoms 
relating to the general state of health often appear. Thus the patient may feel weak, show a disinclination to work, be irritable and somewhat melancholic. Some patients greatly lose in flesh, and present an appearance of suffering, have cold extremities and a slow pulse. Headaches, nausea, and anorexia are also often met with.

Whether these symptoms are due to auto-intoxication as some, especially of the French writers, assume (Bouchard ') is verr difficult to state. It is, however, certain that this theory does not apply to all cases of this kind.

Gastric symptoms (nausea, anorexia, etc.) are as a rule met with only in cases in which the small intestine is affected. If the catarrh is limited to the large bowel these symptoms are usually absent.

Objective Symptoms. - In some cases the abdomen is bloated, especially shortly after meals, and somewhat tender to pressure. There may be tenderness all along the colon; occasionally the ascending colon can be felt as a sausage-like body containing hard masses, which change their shape upon digital pressure, or this part of the colon is filled with gas and liquids and a splashing sound can then be easily eroked. Similar phenomena may be obserred also in the descending part of the colon (S Romanum) in the left iliac fossa. Tenderness along the colon upon pressure is often found; usually the pains are felt just beneath the area where the pressure is exerted; sometimes, however, the pain appears in a more remote spot. Thus, for instance, upon pressing upon the ascending colon in the right iliac fossa, pain is felt across the abdomen in a line lying horizontally at two fingers' width above the navel (transverse colon). Intestinal peristalsis may be observed in persons with thin abdominal walls, especially

'Bouchard: "Leçons sur les Anto-intoxications," Paris, 18si. 
after a palpatory examination. All these signs, however, are occasionally absent.

In the symptomatology of the chronic intestinal catarrh the character and frequency of the stools are of greatest importance. IVhile in acute intestinal catarrh diarrhœe is almost a constant characteristic symptom, there is much variation in the frequency of the dejecta in the chronic form. With regard to this point Nothnagel divides cases of chronic intestinal catarrh into the four following groups:

1. Cases characterized by pronounced constipation. An eracuation appears only once in two, three, or four days; sometimes only with the aid of cathartics. The fecal matter is usually hard. As a cause of the constipation, Nothnagel assumes a decreased activity of the automatic nervous apparatus of the intestines, this being the result of the catarrhal process.

2. Cases in which constipation and diarrhœa constantly alternate. For two or three days there may be a daily evacuation of very hard dejecta. On the following day there may be four to six very thin or mushy movements mixed with mucus, accompanied by violent pains, and then again constipation for a day or two, etc. Or there may be quite normal evacuations (once daily) for a few days in succession and then again four to seven diarrhœal movements in one day, and after this constipation. The principal feature of these cases is the constipation, but the excitability of the nervous apparatus being quite good, the decomposed stagnant contents often cause increased peristalsis and diarrhœa. Sometimes these alternating periods of constipation and diarrhcea continue for a long time. Thus the patient may be constipated for four or five weeks, or even for a few months, and then again the diarrhœa may set in, lasting several weeks or months. 
3. In a very limited number of cases there is a daily evacuation, which is usually not formed and mushy.

4. Cases in which there are for months several diarrhoal evacuations daily. The dejecta as a rule show the biliary reaction, or they may contain yellow fragments of mucus, yellow tinged epithelia, and round cells. In these cases the catarrhal process affects not only the large bowel but also the small intestine. The absorption suffers and there are more abnormal products in the contents (acids), which give rise to increased peristalsis in the small as well as large bowel.

Besides these typical cases there are some in which the nervous element plays a part in combination with the catarrhal process. Thus there are patients who are molested with diarrhoeal movements only during the night or in the early morning hours (morning diarrhoea of Delafield'), while they feel well during the remainder of the day.

The quality of the dejecta in those cases in which there is constipation is almost normal, with the only exception that there is an admixture of mucus. Nothnagel considers this point the most important in the recognition of a catarrhal condition of the intestine. The mucus may be absent in rare instances in which the scybala are small and the layer of mucus within the intestine is very tough and adherent, so that the fecal matter cannot carry it along in its passage.

The quantity of mucus varies greatly. While in most cases only small particles of mucus are found, there are some in which a considerable amount may be passed. Large amounts of mucus without fecal matter are often found in enteritis membranacea, less frequently in chronic enteritis.

'F. Delafield : Medical Record, May 11th, 1895. 
In cases in which the dejecta are more or less thin, mushy or watery, the fecal matter has a light color, brownish-yellow or grayish-yellow, and may at times be rery poor in biliary matters. In these instances, undigested food particles are easily found. Thus small particles of meat or starchy food may be discovered.

The microscopical examination of the dejecta is often very useful, for eren in cases in which macroscopically nothing abnormal can be discovered, the microscope may rereal considerable amounts of undigested meat fibres, starch granules, and fat globules. Such substances, if frequently present, indicate that the catarrhal affection is principally within the small intestine. The microscope here further shows the presence of epithelial cells, sometimes of a yellow color and mostly in a shrivelled condition and embedded in mucus.

According to Rosenheim, ' chemical examinations of the dejecta have no practical value in this affection. The reaction with regard to litmus varies greatly and is dependent upon the frequency of the stools and the quality of the ingested food. As a rule, however, an alkaline reaction is found.

The degree of fermentative processes in the intestines may be gauged by the intensity of the feeling of tension in the abdomen, the frequency of flatus, and the condition of the dejecta. The latter may present a very fetid odor and a foamy surface. If the movements are diarrhoal, a fermentation tube may be filled with the liquid contents and kept at blood temperature for a few hours; the amount of gas developed in the tube will indicate the degree of fermentation. The character of the urine is also of impor-

1Theodor Rosenheim: "Pathologie und Therapie der Krankheiten des Darms, " Wien und Leipzig, 1893 
tance with regard to this point. In conditions in which there is considerable fermentation and absorption of decomposed products within the small intestine, it usually gives a more or less strong indican reaction and also a decided Rosenbach reaction (Burgundy red color after boiling and the addition of nitric acid).

Chronic enteritis complicated with catarrhal ulcers manifests itself by more frequent attacks of diarrhoea, admixture of blood or pus in the dejecta, and pain. All these symptoms are especially apt to be present if the lower part of the intestinal tract is affected; if the ulcer is in the small intestine, diarrhoea is often absent, nor need there be any signs of blood or pus in the dejecta.

Atrophic processes may also accompany the enteritis. If these involve only a small part of the intestinal tract, no symptoms whatever. may result; if, however, larger parts of the small intestine are affected, the absorption of food is greatly impaired and then severe symptoms occur. Diarrhoea without passage of mucus and accompanied by a gradual but steady loss in weight is present, as are occasional symptoms of pernicious anæmia. This condition is found much oftcner in infancy than in later life.

Course.-As a rule chronic enteritis is a very tedions affection. It may last many years, eren until the end of life. The intensity of the symptoms varies a great deal, and there may be periods of apparent perfect euphoria. There always remains, however, a decided weakness of the intestine, which is easily upset by slight errors in diet, which in healthy persons would be harmless.

Diagnosis. - The diagnosis of chronic enteritis is made if there are abnormal sensations within the abdomen, accompanied by irregularity of the bowels and the presence of mucus in the stools. Habitual constipation can be ea- 
sily differentiated from enteritis: (1) by the absence of mucus; (2) by the fact that it does not so easily nor so completely respond to mild cathartics. Malignant growths are often accompanied by enteritis, and thus the symptoms of the latter often give rise to mistakes. A longer period of observation, however, will aid in arriving at a correct diagnosis. In case of a neoplasm symptoms of cachexia will not fail to appear nor will the accompanying enteritis be so readily alleviated as if it were the only affection. In ulcer of the intestine pains predominate and are a marked feature. Constipation and diarrhœea dependent upon disease of the stomach will be recognized: (1) by the absence of mucus in the stools; and (2) by an examination of the gastric contents. They will readily yield to treatment directed toward the gastric disorder.

With regard to the localization of the process, the following is of importance: Chronic inflammation confined to the small intestine is usually accompanied by gastric symptoms, constipation, and the presence of small particles of mucus in the stools, having a yellow tinge and being well mixed with the dejecta. If the large bowel alone is involved (colitis), there is constipation with the presence of more or less mucus of a grayish color, either covering the entire fecal mass or appearing here and there on its surface. Occasionally, especially if the lower part of the bowel is affected, the mucus appears at the end of the defecation and is then voided witlont any admixture of fecal matter. If the inflammatory process involves both the small and the large intestines, constant diarrhoea is a predominant feature. The mucus found in the dejecta has a yellowish color; besides considerable quantities of undigested food are discovered in the fecal matter.

Prognosis. The prognosis of chronic enteritis depends 
upon the intensity of the symptoms, the duration of the disease, and also greatly upon the age and the constitution of the patient. In infancy and in old age chronic catarrh of the intestines nust be considered a grave affection. The same applies to persons with a weakened constitution (tuberculosis, cardiac or other important lesions). A chronic enteritis of intense type which has lasted a long period of time is hardly ever cured perfectly. There may be improvements in the condition of the patient, but relapses are sure to follow soon. Cases of a mild nature, however, often end in recovery, especially under an appropriate treatment. In old age a complete cure rarely takes place. If atrophy of the intestines has developed, then the condition is very unfavorable, the patient succumbing after a period of about twelve to eighteen months.

Treatment.-As in the treatment of chronic gastric catarrh, and perhaps in a still greater degree, hygienic and dietetic measures here play the chief part. It will be at first important to regulate the mode of living of the patient -not too much work, not too great business strain, plenty of outdoor life and exercise, regularity of meals. Exposure to cold should be carefully avoided. The patient should dress warmly, especially the abdomen and feet (flannel bandage around the abdomen), and should be particularly careful not to get his feet wet. In rainy weather shoes with thick soles or rubbers shonld be worn. With regard to diet the following rules are of value: the meals should be taken frequently and in small portions. Indigestible substances should be avoided. Sufficient nourishment should be given, and care taken that there is an increase rather than a decrease in weight. In cases of diarrhoea the following should be forbidden: acid or sweet wines, all mineral waters charged with carbonic-acid gas, lemonade, all 
kinds of fruits, salads, all kinds of cabbage including cauliflower, rye bread, and pastries. Give eggs (soft-boiled or scrambled), light meats, especially sweetbread, calf's brain, spring chicken, steak, lamb chops, oysters, lean fish, white bread well baked or toasted, fresh butter, cream soups, boullon, rice, sago, macaroni, mashed or baked potatoes, milk, cacao, tea. Kumyss, matzoon, ginger ale, good claret or Tokay may also be allowed. As a rule nothing should be taken in large portions, and the drinks should be warm or cool (temperature of the room), but not cold. Large amounts of liquids should be avoided. Patient with very severe symptoms (frequent diarrhœa, intense pains, great weakness) must be kept abed for a short time and put on a rigorous diet at first, as in cases of acute enteritis. Upon improvement of the condition the dietetic rules described above should be followed.

In cases attended with constipation the diet may be more liberal. Besides all the articles of food mentioned in the diarrhceal group, light fruits, as oranges, grapes, ripe pears, and green vegetables, green peas, cauliflower may be added. The ingestion of large amounts of starchy foods, easily assimilated fats, butter, cream, and of fluids is very beneficial. The more indigestible articles of food, like bran breads (pumpernickel), sausages, lobster salad, mayonnaise dressings, cabbage, cucumbers, etc., should be avoided. Beer, ale, Rhine wine taken moderately are permissible.

Hydrotherapeutic Measwres. - In cases of diarrhœea warm mineral baths or baths with the addition of pine needle extract and mud and bran baths are favorable. Cold baths should be avoided. A cold sponge bath, however, or a cold shower on the back may be serviceable in chronic enteritis with nervous symptoms. A Priessnitz (wet pack) 
over the abdomen may be advantageously used.over night. Cold sitz baths and cold showers over the abdomen are also often beneficial.

Mineral Waters. - According to Nothnagel chronic enteritis is sometimes greatly improved, and even perfectly cured, by a methodical course of drinking certain mineral waters. Such a cure can best be carried ont at the mineral springs themselves. For here the patients not only take the waters in the. right way, but also observe the necessary rules of diet and are besides kept free from their business cares. Carlsbad is to be regarded as the best place in cases of chronic enteritis in which the diarrhoea is a prominent feature; Vichy comes next. For cases of chronic enteritis with constipation Marienbad seems to be very useful; the same applies to Saratoga (Hawthorn and Congress Springs). For cases in which neither constipation nor diarrhoea plays a prominent part Kissingen or Homburg may be recommended. Chronic enteritis accompanied by anæmia may be benefited at the watering-places of Franzensbad and Elster. The Carlsbad water should be taken in small quantities, about a wineglassful twice daily; in some cases even smaller amounts (25 to $50 \mathrm{gm}$.) three to five times daily. In cases which have been benefited by a drinking cure in Carlsbad, Nothnagel suggests having these patients use at home the Carlsbad waters in a similar manner as at this resort, four times a year for an entire month. Nothnagel says: "The chronic condition requires a chronic treatment."

Medicaments.-Strong catharties should be avoided in the treatment of the constipation. Here some articles of diet which moderately increase the intestinal peristalsis may be first tried-buttermilk, a glass of cold water, stewed fruits, and the like. If these fail, small amounts 
of rhubarb, fluid extract of cascara sagrada, podophyllin may be used. Of greater value, however, are rectal injections either of plain water or with the addition of some soap or salt (a teaspoonful to a quart) or Carlsbad water. Enemas of olive oil, as first recommended by Habershon and later by Kussmaul and Fleiner, may also be advantageously used. The oil enemas should, however, be given in small quantities (half a pint to a pint) and be retained over night in the bowels. The frequent use of calomel, castor oil, and jalap should be forbidden.

The diarrhoea is best treated either by large doses of subnitrate of bismuth or salicylate of bismuth ( 1 to $2 \mathrm{gm}$., gr. xv.-xxx.) three times daily, or some of the drugs containing tannic acid as their principal ingredient (calumba, cascarilla, rhattania, catechu, kino, lig. campechianum, fructus myrtili). Weber' recommends the following prescription:

R, Extr. monesiæ,

Extr. calumbæ................... āa 15.0 ( $\xi_{\text {ss. })}$

Extr. gent. et pulv. liq................. q.s.

Ut f. pil. cxx. S. Three times daily two to four pills.

I very frequently give fluid extract of condurango and fluid extract of calumba of each twenty drops three times daily. Dermatol (subgallate of bismuth) seems to be quite beneficial in cases in which the formation of gas is a predominant feature. It may be given in doses of half a gram (gr. viii.) three times daily. For the same condition salicylate of bismuth, benzonaphthol, and creosote in small doses may be given. Tannigen and tanalbin may be used in doses of 0.5 to $1 \mathrm{gm}$. (gr. viii.-xvi.) three times daily, the first being preferable. Both substances seem to lessen fermentation, and by their astringent qualities exert a

' L. Weber: New-Yorker medicinische Monatsschrift, 1892. 
beneficial influence upon the healing-process. They may therefore be given continuously for a long period of time.

Cases accompanied by pains will require an opiate (morphine, or still better codeine), with or without the addition of belladonna extract. In chronic proctitis suppositories of opium and belladonna extract with cacao butter are indicated. Small enemas of starch solution with an opiate are also useful here. It is of course to be understood that the administration of opiates will have to be limited to a short period of time. 


\section{CHAPTER IV.}

\section{DYSENTERY.}

Synonyms.-Enteritis crouposa et necrotica; Amœbic dysentery.

Definition.-An infectious disease characterized by specific ulcerations of the large intestine, giving rise to frequent bloody, mucous, or purulent dejections accompanied by tenesmus and general symptoms.

Etiology. - Dysentery occurs under three different conditions: (1) As a disease principally during the warm season in temperate climates, appearing in local epidemics; (2) endemic in hot climates; (3) epidemic at certain times in all latitudes, being quickly disseminated, and also sporadic. While the endemic zone of dysentery is limited to places lying south of the fortieth degree of latitude, epidemics of the disease have occurred in almost erery part of the globe. Dysentery is one of the oldest diseases known. ' It was observed by Hippocrates and well described by Aretrus and Celsus. Aretreus already recognized the ulceration of the intestines in dysentery.

Various causes have been adduced to explain the origin of the disease, and meteorological influences hare been held responsible for its prevalence in local epidemics. The endemic dysentery of the tropics was generally ascribed to the combined action of heat and of the miasm of swamps. Sudden exposure to cold, eating of bad and spoiled food, and the use of stagnant or marshy water were all believed 
to. be factors in producing dysentery. It is only within recent years that its infectious and also contagious character has been recognized. Sodré ${ }^{1}$ says: "A careful etiological study shows that dysentery in whatever latitude it be observed is always due to the action of the same exciting cause, that it starts and is propagated always under the influence of infection and contagion, and that it should be included in the group of parasitic diseases." The exciting cause of dysentery often lies in the soil, in circumscribed foci of infection. These foci are represented by marshes and bogs which receive the drainage from dung heaps and cesspools, or by a soil impregnated with human dejections. The contagious character of dysentery is best shown by the following report of Dr. Beauchef." This writer states that the French ship Loreit, anchored on the west coast of Africa, was in the best possible sanitary condition, not one of the crew being ill. She was then ordered to transport to Goréa the sailors of the sloop of war Eagle, among whom were twenty-nine dysenteric patients. A few days afterward, while on the high sea, dysentery spread among the crew of the Loreit and ceased only after all the patients had been landed at Goréa.

Among the causes which contribute to diffuse the dysenteric contagion and to produce the disease in an epidemic form the following may be mentioned: Crowding together of individuals, the vicissitudes of war, bodily privation, chiefly hunger. These factors are frequently found associated in times of war when epidemics of dysentery have often appeared, causing great ravages.

Since bacteria have been found to play an important

1 A. Sodré: “Dysentery, " Twentieth Century Practice of Medicine, vol. xvi., p. 241.

1 Beauchef : Cited after Sodré, loc. cit. 
part in the etiology of infectious diseases, many investigators have tried to discover the particular micro-organism producing dysentery. Various bacilli and cocci have been described and held responsible as etiological factors, but their relative significance has not been determined. As early as 1859, however, Lambl ${ }^{1}$ called attention to the presence of amobr in the intestiual contents. He found them in the stools of a child suffering from dysentery. Loesch, ${ }^{2}$ in 1875, observed amobre in the dejecta of a patient suffering from chronic dysentery. He was the first to attribute the disease to this micro-organism. He also succeeded in experimentally producing a dysentery-like disease in a dog to which he had administered rectal injections of fecal matter containing amcbr. The observations of Loesch have been confirmed by Koch, ${ }^{3}$ who, while investigating dysentery in Egypt, found in post-mortem examinations numerous amcbre in the intestine at the base of the ulcers. The next important contribution on this subject was made by Kartulis, " who, while practising in Alexandria, had an opportunity to observe several hundreds of cases of dysentery. In more than five hundred post-mortem examinations he found the amoebr constantly in the freces and on the surface of the ulcers, and in the abscesses of the intestine as well as of the liver. In other affections of the intestines Kartulis failed to detect the amœba parasites. He also succeeded in cultivating them in infusions of sterilized dry straw, and twice produced dysentery in

${ }^{1}$ Lambl : "Beobachtungen und Studien aus dem Franz-Josef-KinderSpital," 1860.

2 Loesch: "Massenbafte Entwickelung von Amoeben im Dickdarm." Virch. Arch., Bd. lxv.

${ }^{3}$ Koch : Cited after Sodré, loc. cit.

"Kartulis: "Zur Aetiologie der Dysenterie in Aegypten." Virch. Arch., Bd. 105, 1885. 
cats by inoculation with these cultures. Kartulis, therefore, declared the amœbre to be the true etiological factor of dy'sentery.

Very soon afterward observations of a similar character were made both abroad and here. Thus Quincke and Roos, ' Hlara, " Massaiutin, ${ }^{3}$ Nasse, ${ }^{4}$ and others abroad, and Osler, ${ }^{5}$ Stengel, " Musser, Eichberg, ${ }^{7}$ Stockton, ${ }^{\circ}$ Councilman and Laflemr, ${ }^{10}$ and Harris ${ }^{11}$ of this country have also described cases of dysentery with the presence of the amœbre parasites.

The theory of the amøebic origin of dysentery has been disputed by some writers, for they have found this micro-organism in the freces in other intestinal disorders and, in some instances, even in the stools of healthy persons. Thus Schuberg "says: "The abundance of amcebre in dysentery is the effect and not the cause of the disease, the ulcerative lesions affording this habitual denizen of the intestines more farorable conditions for its derelopment." The consensus of opinion, however, is that while harmless amcebr may occur in the intestinal tract, there exists a pathogenic variety of this organism which is specific for dysentery. For this reason Councilman and Lafleur proposed the name

${ }^{1}$ Quincke und Roos: Berl. klin. Wochenschr., 1893.

${ }^{2}$ Hlava : Centralbl. für Bacteriologie, $188 \%$.

${ }^{3}$ Massaiutin : Ibid.

4Nasse: Deutsche med. Wochenschr., 1891.

${ }^{5}$ Osler : Bulletin of the Johns Hopkins Hospital, 1890.

${ }^{6}$ Stengel : Medical New's, November 15th, 1890.

'Musser: University Med. Magazine, December, 1890.

8 Eichberg: Medical News, August 22d, 1891.

19 Stockton: International Clinics, 1894 , i.

10 W. J. Councilman and H. A. Lafleur : "Amœbic Dysentery." Johns Hopkins Hospital Reports, vol. ii., Nos. $7-9,1891$, p. 395.

"K. F. Harris: "Amœbic Dysentery." American Journal of the Medical Sciences, 1898, p. 384.

${ }^{12}$ Schuberg : Centralbl. für Bakteriologie, 1893. 
of amœbre dysenterire for this special variety. The presence of the amøere in the contents of abscesses of the liver, which are so often met with in dysentery according to Sodré, constitutes a powerful argument in favor of the amobic etiology of the disease.

It is generally believed that the amobre enter the system along with the food or drink. Sodré believes that they cau be taken in with the air. Certain waters, however, apparently constitute the principal means of propagation of these amobr. Thus, Barthelemy ${ }^{2}$ relates that the troops when operating on the shore of the Ouemé, whose clear running water was filtered in Chamberland filters before being used, were in good health and free from dysentery; when, however, the army mored away from the Onemé in the direction of Abomey they were compelled to use unfiltered swampy water. From that moment dysentery made its appearance. Fitz and Gerry ${ }^{2}$ described a case of dysentery with the presence of amober in the stools and found the same micro-organisms in a cistern, the water of which the patients constantly used.

Agre does not seem to have any influence upon the disease. Statistically a greater number of eases is found among iddults, as these are more exposed to the morbific causes. Both sexes are equally predisposed to dysentery, and no race enjoys immunity from it. One attack does not confer immunity against others. Persons who suffer from want of food or who live on food of bad cruality are most liable to contract the disease. Harris say's: "Dysentery is a disease pre-eminently of the poor, and is almost always associated with filth, bad hygienic surrolindings, and lack. of proper food." This statement, however, is somewhat

'Barthelemy : "Medical Report of the IVar of Dabomey "

:Fitz and Gerry: Cited after Sodré, loc. cit. 
too categorical, and I fully agree with Sodré, who remarks that dysentery is observed also in persons of the wealthier class, who live on the best food and are surrounded with every comfort. Nevertheless, it must be admitted that it is most frequent among the poor, and chiefl $r$ among people who live under bad hrgienic conditions.

Morbid Anatomy. - In acute drsenters the large intestine is almost always found in a thickened condition. This thickening involves all the intestinal coats, but is most markerl in the submucosa. Sometimes the latter laver alone is involred. The mucosa, when washed with water, presents a bright red, at some places dark red color. The folds of the mucosa are much more roluminous than normally, and thus present considerable prominences. Small red nodules of rarious size are also seen scattered orer the mucons membrane. Besides these nodules more or less numerous ulcers are found. These rary greatly in size (from a pinhead to two inches long) and also in depth, some being superficial, others quite deep. The nlcers are situated chiefly on the folds of the mucosa. Ordinarily they are oblong and lie transrersely to the long axis of the bowel. Sometimes ther are circular, sinuons, or irregular.

Councilman and Lafleur hare described on the surface of the mucosa sharply outlined projecting nodular thickenings, in which are obserred carities filled with a gelatinous mass communicating with the surface of the mncous membrane by small openings, frequently not larger than the head of a pin. These writers hare also pointed out as characteristic of the dysenteric ulcers their undermined edges. The disease process in drsenters, accorling to Councilman and Lafleur, is essentially one of adrancing infiltration and softening of the submucous and intermus- 
cular tissue with subsequent necrosis of the overlying tissue. The amœbre reach the submucosa without injuring the mucous membrane. Here the essential changes are first produced, and the mucous membrane is interfered with later. The mucosa becomes œedematous and ruptures after a while, forming an ulcer.

Harris described two anatomical forms of ulcers found in dysentery. In the first form, which is encountered most frequently and can be considered as the typical intestinal lesion of the disease, changes in the submucosa rasy be traced in advance of the surface ulceration for quite a distance, thus undermining the comparatively healthy mucosa above. In the second form the ulcers increase in size by gradual softening and breaking down at the surface, nerer by necrosis and sloughing of the underlying tissue. Ulcers of the second category occasionally do not penetrate deeper than half-way through the mucosa. Generally they extend into the submucosa. They never contain amobæ.

The lesions described are usually found throughont the entire large bowel, but as a rule they do not extend beyond the ileocecal valve. In a comparatively small number of cases the small intestine is also involved, principally the ileum.

In some instances gangrene of the intestine is found. Many authors even describe a gangreuous form of dysentery. Sodré, however, does not regard the gangrene as a lesion brought on by the amobre dysenterix, but by the action of bacteria foreign to the dysenteric process. According to this author gangrene is a complication of dysentery, but not a specific lesion. In this complicated form, besides the ulcers described above, there exist others of a gangrenous character. The gangrenous process may also extend beyond the ulcers. On the brownish-red mucosa 
gangrenous patches of a dark color and of various size are seen. In this condition the mucosa may be detached over a considerable area and eliminated with the dejections.

In chronic dysentery the intestine is pale with slate colored spots. Its walls are thickened. The mucosa presents a pale rosy or slate color. Ulcers in different stages of derelopment are encountered. Often the ulcers occur in groups separated from each other by more or less extensire healthy areas of intestine. Sometimes in certain portions of the intestines the ulcers become confluent. Dysenteric nlcers may be round, elliptical, or serpentine in form and usually have thickened and callous edges. In the neighborhood of the ulcers, there is no hyperæmia or cedema, although an increase of fibrous tissue is noted. Undermined ulcers undergoing a process of repair are also found. The mucosa glands are found dilated and filled with mucus. In some places glandular cysts of considerable size are encountered, in others the glands hare almost disappeared, and only traces of them are left. The mucosa is thickened and filled with round cells. The submucosa is likewise thickened and in some places cedematous. Dense fibrous tissue is found almost all over in this layer, predominating, however, at the location of the cicatrices and of ulcers in the process of repair.

In both the chronic and the acute form of dysentery, but principally in the latter, besides the lesion of the intestines described above, the liver is frequently found diseased. In dysentery complicated with gangrene this organ is usually greatly increased in rolume, tumefied, soft, and friable. The cross-section presents a dark color interspersed with yellowish spots. The latter are usually somewhat raised above the surface. On microscopical examination the hepatic cells show a large amount of fat; besides, 
small round abscesses are found around the capillaries, which are most probably due to emboli.

Aside from these very small pus collections of pyæmic origin, other abscesses are found which differ from these by their size and the nature of their contents. They are the so-called dysenteric abscesses of the liver, and are most often encountered in acute dysentery without gangrene. The dysenteric abscesses vary greatly in size from a few lines to several inches. They are situated chiefly in the right lobe of the liver near the surface. Often several are found together. The contents of these abscesses vary greatly. In the most recent, the abscess does not empty itself on section. A small amount of glairy, semi-transparent fluid exudes and leaves behind an irregular spongelike mass, the fluid being apparently held in the meshes. In the older abscesses the contents are more fluid, the latter having a greenish opaque color. In these are suspended some solid masses of tissue. In some instances the contents are brownish or streaked with brownish-red from admixture of blood. Microscopical examination of the content; of the abscesses reveals the presence of a few pus cells, a large quantity of fatty granules, necrotic hepatic cells, a few blood corpuscles, a great number of amcebre (see Fig. 28), and sometimes micrococci and bacilli. According to Councilman and Lafleur, there is no definite abscess-wall, the liver tissue passes gradually into the abscess, and the contour of the edge is very irregular, sometimes extending into the liver for a distance of several nodules. The abscess may penetrate the capsule of the liver and either open externally or it may burst into some of the adjacent organs, as, for instance, the lungs, the stomach, the intestines, or the peritoneal cavity. Most often, however, it bursts into the lungs. 
Symptomatology of Acute Dysentery.--The disease may begin suddenly without any premonitory symptoms, or after a few days of general malaise, loss of appetite, and irregularity of the bowels, the patient is attacked with abdominal colic and diarrhoea. These symptoms are usually accompanied by chills, vague pains through the body, and fever. The stools, at first abundant and watery, very soou become scanty, mucous, and usually contain

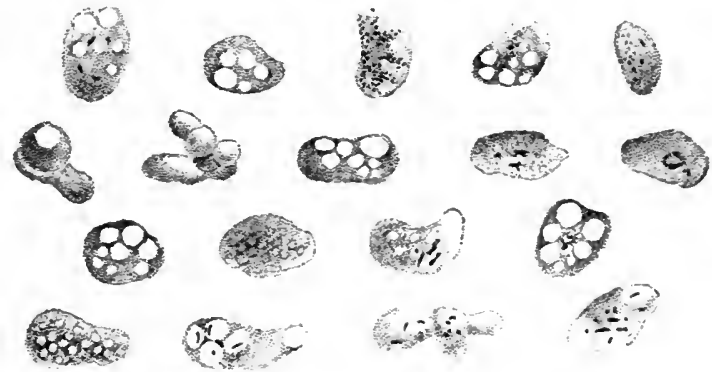

Fig. 28.-Amøba from an Abscess of the Liver. $\times$ i50. (Sodré.)

blood. Gastric disturbances are present in almost all cases: anorexia, nausea, often vomiting. The principal features of dysentery are the characteristic stools, the abdominal pains, and tenesinus.

1. Stools. The evacuations increase in frequency, occurring from twenty to twenty-seren times during the twenty-four hours. The calls to stool are usually preceded by rumbling and colicky pains, and are followed by straining and tenesmus. While during the first and perhaps the second day of the disease the motions are copious, they soon become scanty. The patient is then able to expel but a small quantity, about a teaspoonful of mucus mixed with blood, after painful efforts. Occasionally a few small pieces of fecal matter are passed. The dejecta ocussionally change their character with regard to frequency as 
well as consistencr. Intermissions and exacerbations of the diarrhoea are sometimes obserred in the conrse of the disease. The mucus in the stools is almost alirars mised with blood. In some cases the dejecta are hemorrhagic, that is, consist of almost pure blood, either red and fluid or dark and coagulated. In dysentery complicated with gangrene the stools are serous, of a dark reddish-brown color, and contain, in addition to finely dirided membranous threads, large and thick masses of necrotic tissue of a gray or black color. The gangrenous dejecta have an intensely offensire odor. In many instances the stool contains no bile.

Amcebe are almost always found in the drsenteric stools, especially if the lesions are quite extensive. In examining the freces for amobre it is well to use some precaution. If possible the examination should be made immediately after the dejecta hare been passed. If this be impossible, the stool should be preserred in a clean ressel and kept in a warm place until the examination is made. The amcebr are from 12 to 36 : in diameter, and when alive frequently change their shape by contracting some part of their bodies in order to more about. The body of these micro-organisms consists of an outer clear homogeneous substance or ectosarc and an inner highls refractive mass or endosare. Within the latter are usually found some bacteria, sometimes changed red blood corpuscles, and a few quite large racuoles. The amobre, when outside of the intestinal tract, die rers quickly, especially if ther are kept in a cool place. When dead, these organisms generally show a round or almost round configuration.

(2) Abdominal pain. Abdominal pains exist with greater or less sererity in almost every case. The pains may be experienced continuously, or principally before an evacu- 
ation. Most often they are located in the umbilical region and in the left iliac fossa, but sometimes they exist in the right iliac fossa and mas then almost simulate an attack of appendicitis. The pains mas be so serere that the patient is forced to lie perfectly still for fear of increasing them. Pressure exerted on the large intestine as a rule prorokes more or less intense pain. According to Dutroulean, ${ }^{1}$ in some rery grave cases there is a total absence of colic during the entire course of the disease.

(3) Tenesmus. Rectal tenesmus, consisting at first in painful sensations of pressure and constriction and later in an intense desire to go to stool, is encountered rerr frequently. In grare cases of drsentery the tenesmus mar exist almost uninterruptedly. Off and on the patient succeeds in expelling a small amount of fecal matter or slime or merely gas, and then feels reliered for a short while. Very soon, howerer, the pains in the anal region return with the same sererity. When the tenesmus is rery serere it mar be accompanied by drsuria or strangurr. In this condition the patient presents a pitiable appearance. His straining is frequently agonizing and oceasionally accompanied by fainting.

Besides the three cardinal symptoms of dysentery just described, other ssmptoms are often encountered. Ferer may be present, especially in the sererer form of the disease. It mar occur in the form of chills, when the disease is first ushered in. As a rule, the fever is not rery high and shows an irregular course. Gastric symptoms are often present. Ther consist in intense anorexia, nausea, romiting, and pain in the epigastric region. The general condition is more or less affected according to the sererity

'Dutrouleau: "Traité des Maladies des Européens dans les pass chauds, " Paris, 1868. 
of the disease. In grave cases prostration is marked, the skin is dry, the features are altered, and the extremities sometimes cold. The pulse is small and rapid. Sometimes cerebral disorders, stupor, drowsiness, even delirium, are encountered.

Dutronlean and others divide cases of acute dysentery into three groups: Cases of a mild character, those of medium intensity, and those of a severe type. In the mild form, there exist only local symptoms which are usually not very intense. In the form of medium intensity, the local symptoms are more accentuated and general symptoms are encountered. In the severe form, there are fever, inteuse pain, very bloody stools, great prostration, and intolerable tenesmus.

Simmptomratology of Chronic Dysentery. - Chronic dysentery develops either after several attacks of the acute form or directly from the first acute attack, which after some periods of improvemeut persists to a greater or less extent. Cases of chronic dysentery are also divided into three categories:

(1) The mild form. The general nutrition is not interfered with. 'The patieuts usually complain of slight constipation interrupted by light attacks of diarrhoea. Tenesmus is either entirely absent or present in a very slight degree. Even during the attacks of diarrhcea the passages are, as a rule, not bloody.

(2) Form of medium intensity. Here slight gastric symptoms are present, like anorexia, belching, etc. The general condition is interfered with to a considerable extent. There are almost always periods of intermission and exacerbation of the disease. The patient may have regular movements or be slightly constipated, for a period varying from a week to ten days, but soon diarrhcea appears and lasts 
for four or five days. The stools are then watery, contain mucus, and-occasionally a little blood. Slight colicky pains are present, as well as moderate tenesmus and a sensation of heat or burning in the rectum.

(3) The severe form. General nutrition is greatly impaired. The patient becomes emaciated, pronounced gastric symptoms are present: anorexia, a bad taste in the mouth, often nausea, occasionally vomiting. As a rule, there is persistent diarrhoea, and the dejecta present a mucous or muco-sanguineous character. Colicky pains in the abdomen and pronounced tenesmus are present. In some cases, however, the diarrhœe alteruates with short periods of constipation lasting two or three days. The patient usually feels very weak and is obliged to stay abed a great deal of the time.

Course.-The course of acute dysentery is very indefinite. Sometimes the disease terminates in recovery in eight to fifteen days; sometimes in one to three months; sometimes again death occurs a few days after the commencement of the disease. Again, a case of dy sentery may at first be mild, but later assume a dangerous character, and even terminate fatally. Intermissions and exacerbations are often encountered in this disease. When dysentery becomes chronic its duration varies greatly, often depending upon the severity of each particular case. Thus, it may last five to six months or many rears. Even in the chronic form recovery is not entirely impossible.

Complications. - The course of the disease is occasionally modified by various complications. Peritonitis often results from an extension of the ulcerative process from the intestinal wall to the peritoneum. Perforation of the intestine may occur in a similar way, and is observed principally in gangrenous dysentery. Sudden death is 
occasionally observed in such an event. In acute as well as in chronic dysentery serere hemorrhages from the bowel may take place. The loss of blood may be so great even as to cause death. Thrombosis of the femoral artery as well as of the venous sinuses of the brain has been observed by Laveran ' as a complication of dysentery. A patient of mine with acute dysentery, apparently on the road to improvement, suddenly oue day developed a paralysis of the upper and lower right extremities. He later lost consciousnes and died about forty-eight hours after the first signs of paralysis. Here most probably thrombosis of some brain ressels took place.

The most frequent complication of dysentery is abscess of the liver. In the majority of instances it is observed in convalescence from acute dysentery or during the erolution of chronic dysentery. The symptoms of the formation of an abscess in the liver are: fever of an irregular character, occasionally chills and pain in the hepatic region which may radiate to the right shoulder. The physical examination often reveals some enlargement of the liver. In the event of a liver abscess opening into the lungs, there is persistent cough and sometimes expectoration of a reddish-brown fluid containing amœbr. Abscess of the liver is more frequently encountered in tropical regions than here. The course of such an abscess is very irregular. Sometimes it progresses rapidly, at other times it shows periods of intermissions and exacerbations. The large abscesses of the liver, if not operated upon, usually terminate in death. Rarely recovery may follow the opening of the abscess into a neighboring organ.

${ }^{1}$ Laveran: "De la phlébite, de la thrombose et des paralysies comme complications de la dysentérie." Archives de Médecine militaire, 1885. 
Diagnosis. - The diagnosis of acute dysentery is usually very easy. The symptoms above described, being ordinarily present, cannot fail to indicate the disease. The most reliable evidence is afforded by the character of the dejecta, the presence of mucus, an admixture of blood and pus corpuscles. Appendicitis is occasionally simulated by dysentery if the pains involve principally the appendicular region. Usmally, however, it will be found that, besides the tenderness over the appendix, there are also similar areas of pain over other portions of the large bowel, especially in the left iliac fossa. Besides, the character of the stool will help to reveal the true condition.

The diagnosis of chronic dysentery is usually somewhat more difficult. Repeated examinations of the freces will, as a rule, reveal the presence of amobre at one time or another and thus aid in discovering the disease. Many diseases of the rectum, as for instance proctitis, rectal polypus, and cancer, often present symptoms similar to those of chronic dysentery. A careful local examination, however, will clear up the diagnosis without difficulty.

Prognosis. - Dysentery must always be considered a quite serious disease. Even the mild form is at times liable to assume a dangerous character. On the whole dysentery must be regarded as a treacherous and insidious malady. In general it must be said that cases of sporadic dysentery or of the epidemic form appearing in the cold and temperate zones take a much milder course and thus present a more farorable prognosis than does the endemic dysentery of hot climates. These remarks apply to both acute and chronic dysentery.

Treatment of Acute Dysentery. - The patient must be kept abed and put on a diet consisting of liquid food (milk aud strained barley water, bouillon, bouillon with egg, egg 
water, tea). Ipecacuanha has been found of great benefit in this disease. It may be given, according to Sodré, in the following combination:

Powdered ipecacuanha............... 0.1 (gr. ij.)

Powdered opium.................. 0.02 (gr. $\left.\frac{1}{3}\right)$

Calomel........................ 0.05 (gr. $\left.\frac{8}{4}\right)$

In capsules, one to be taken every two bours.

In case the evacuations contain very small quantities of fecal matter, it is best to give a cathartic, as a large dose of castor oil (one to two tablespoonfuls) or sōdium or magnesium sulphate one teaspoonful twice during the day. The purgative, however, should be administered only on the first or second day of the disease, and not be kept up for a long time. In order to allay the pains, hot poultices are applied over the abdomen and opium is administered. Thus, Dorer's powder may be given in three-grain doses every two or three hours. This medicament may also be combined with salol, subnitrate of bismuth, tannigen, tannalbin, etc. The tenesmus, if severe, must be subdued by suppositories containing opium and belladonna, and by washing out the bowel with a quart of water containing a teaspoouful of essence of peppermint, which can be done once or twice in twenty-four hours. Astringent solutions have been recommended as injections for the large bowel. They are not, however, of great benefit in acute dysentery. Besides the points just mentioned, the condition of the patient must be carefully watched and erery complication treated by itself. The high fever may necessitate the use of an antipyretic; the weak action of the heart analeptic drugs, etc. As soon as the severe symptoms are allayed and the patient is on the way to recovery the diet can be cantiously increased.

Treatment of Chromic Dysentery. - If the patient is living 
in an endemic centre of dysentery, it is best to send him to another climate. The hygienic surroundings of the patient should be carefully selected. The food should be well prepared. The patient should eat often, not too much at a time, and should avoid all coarse and highly seasoned substances. Tannigen gr. viii. three times daily or benzonaphthol in the same dose, or subnitrate of bismuth gr. xxx. t.i.d., can be advantageously given. Sometimes these drugs are combined with codeine or opium. Here local remedies play a prominent part. Loesch was the first to recommend injections into the bowel of solution of quinine $(1: 5,000)$; taunic acid, nitrate of silver, permanganate of potassium have also been employed in clysters with good results. Harris very recently recommended the use of hydrogen dioxide. The ordinary commercial hydrogen dioxide is diluted from four to eight times with water and the solution injected. About a quart is injected twice daily for about a week and then gradually decreased. Harris has seen very good results from this mode of treatment. In cases in which there is an exacerbation of the disorder, the same mode of treatment may be required as in acute dysentery. 


\section{CHAPTER V. \\ ULCERS OF THE INTESTINES.}

\section{DUODENAL ULCER.}

Synomyms.-Round duodenal ulcer; Ulcus duodeni pepticum (Leube).

Definition.-A defect in the mucous membrane of the duodenum.

Etiology.-The etiology of duodenal ulcer corresponds with that of gastric ulcer. It is undoubtedly caused, as in the stomach, by the action of the acid gastric juice upon the duodenal mucosa, the vitality and nutrition of which have been previously impaired. Such conditions occur as a result of circulatory derangements of various kinds. Thus, affections of the lungs and heart or of the liver, an atheromatous state of the duodenal artery may be the positive factors in disturbing the circulation of the mucous membrane. Burns of the skin are an etiological factor which, while not operative in gastric ulcer, is of great importance in duodenal ulcer. After extensive scaldings of the skin, quite often one or several duodenal ulcers appear. According to Mayer ' ${ }^{\prime}$ these ulcers develop from seven to fourteen days after the burn, very seldom much sooner. The primary cause of these ulcers is not yet known. The toxic theory which is the most plausible has been discussed above.

Duodenal ulcer is much less frequent than gastric ulcer.

1 Mayer : Annal. de la Soc. de Méd. d'Anvers, 1865. 
Willigk ${ }^{1}$ found it twice in sixteen hundred antopsies. According to this writer, there are thirty-eight gastric ulcers to one duodenal ulcer. According to Starke, ${ }^{2}$ lowever, the ratio is twelve to one. Kraus ${ }^{3}$ found that the frequency of duodenal ulcers varies in different eountries in a similar manner as does gastric ulcer, the northwestern part of Europe having the highest percentage, while it is but rarely met with in the eastern part. In Kraus' experience duodenal ulcer most frequently occurs in persons between thirty and sixty years of age. Next in frequency comes the very early age (one to ten, and especially infancy). This is another point of difference between gastric and duodenal ulcers, for the former hardly ever occur in children. With regard to the distribution of duodenal ulcer among the sexes, Kraus found it much more prevalent among the male than among the female sex, the relation being ten to one. According to Lebert, ${ }^{4}$ however, the proportion is only four to one. This again is another point of difference in the etiology of duodenal and gastric ulcers, for the latter, as is well known, are much more frequently encountered in women than in men (two to one).

Morbid Anatomy. - A duodenal ulcer resembles in most particulars a gastric ulcer. It is a defect of the mucous membrane having an oblong and oval contour and extending into the depth of the mucosa in form of a terrace or funnel. The ulcer presents an irregular shape only in those instances in which sereral ulcers have coalesced, thus forming one large defect. The size of the ulcer varies from that of a lentil up to that of a dollar. The margins

1 Willigk : Prager Viertel jahresschr., 1833.

2 Starke: Deutsche Klinik, $18 \% 0$.

3 J. Kraus: "Das perforirende Geschwilr des Duodenum," Berlin, 1865.

4 Lebert: "Die Krankheiten des Magens," 1878. 
are usually smooth and overlapping, the latter being especially the case in chronic affections. The base of the ulcer is formed either by thin layers of the remaining intestinal wall, or, if perforation has taken place, by adhesions with neighboring organs.

Situation of the Ulcer.-Ordinarily the ulcer is found in the ascending or the upper horizontal part of the duodenum, much more rarely in the descending part, and only exceptionally in the lower horizontal section. As a rule it is situated immediately behind the pyloric fold, rarely at some distant point. If the ulcer is situated in the descending part of the duodenum, especially in the immediate neighborhood of the diverticulum Vateri, it may cause through cicatricial strictures important complications involving the pancreatic and biliary outlets.

As a rule there is one duodenal ulcer, exceptionally there are two or four. In the latter instance the ulcers may be found in different stages of development: in the initial stage, in that of commencing cicatrization, or fully cicatrized. The cicatricial process may lead to manifold complications. A stenosis of the duodenum just behind the pylorus or at some distance may result, and create exactly the same disturbances of the stomach as are found in cicatricial stenosis of the pylorus itself. I had the opportunity of observing two cases of this kind. In both the diagnosis of a benign stricture of the pylorus had been made and the patients subjected to operation. At the laparotomy the stricture was found in the dnodenum, in one case immediately behind the pylorus and in the other at some distance therefrom.

Sometimes the ulcer progresses quickly and leads to perforation into the peritoneal cavity. Death from shock or from diffuse peritonitis then occurs. If there is a slow extension of the ulcer, it often gives rise to circumscribed 
peritonitis, usually with adhesions to neighboring organs. If the ulcer perforates after adhesions have been formed, it usually leads to an encapsuled purulent peritonitis. The ulcerative process may occasionally extend to contiguous parts with the formation of ulcers in the liver, gall bladder, or other neighboring organs. The development of a cancer at the base of a duodenal ulcer has also been observed $b_{\text {: }}$ Eichhorst ' and Ewald. ${ }^{2}$

Symptomatology.-Occasionally there may be no symptoms whatever during life and the duodenal ulcer may not be discovered until at the autopsy. Sometimes there are no symptoms at first, then suddenly the disease manifests itself by a severe and dangerous hemorrhage or by a fatal perforation. In the majority of cases, however, there are pronounced manifestations during the existence of a duodenal ulcer. Most frequently pains are present, usually to the right of the linea alba, extending up to the right parasterual line in the region below the liver. These pains usually appear from half an hour to two or three hours after meals; as a rule they do not radiate to the back but rather somewhat downward in the abdominal cavity. While the pyloric region is often found slightly painful on pressure, there is no circumscribed area in the epigastrium intensely painful on deep palpation as in ulcer of the stomach. In rare instances the pains are felt by the patient in the epigastric region, which may also show tenderness on pressure. Dyspeptic symptoms, as for instance loss of appetite, nausea, fulness in the epigastric region, are as a rule absent. Vomiting is likewise a rare occurrence in simple duodenal ulcer, which has not gone on to a partial stenosis of the intestinal lumen.

1 Eichhorst: Zeitschr. f. klin. Medicin, Bd. 14, p. 522.

${ }^{2}$ C. A. Ewald : Berl. klin. Wochenschr., 1886. 
Hemorrhages as the consequence of an erosion of a more or less large blood-ressel, through the progressing necrotic process, occur in about thirty per cent of duodenal ulcers. The blood is frequently voided with the stools (melæua) which appear dark red or tarry. Occasionally, however, there may be vomiting of blood (hæmatemesis), in connection with the melæna or without it. If the hemorrhage is very great the patient may bleed to death. This, however, is rare; as a rule the patients recuperate from the loss of blood in about the same time as they do from a gastric hemorrhage.

Constipation is often present. The general condition of the patient is usually good and there may be no loss in flesh.

Perforation is quite a frequent event in duodeual ulcer. The symptoms will differ according to whether perforation has taken place before or after adhesions have been formed. In the former instance perforation leads to a general peritonitis, ending fatally in eighteen to thirty hours. Rarely the course is more protracted when the inflammatory process of the peritoneum has not assumed large dimensions and has become quickly localized through the formation of adhesions in the neighborhood. The perforation manifests itself by a sudden appearance of intense pains in the abdominal cavity, by the usual signs of a general collapse (cold extremities, very quick pulse), and by a swelling of the abdomen. The patient presents an expression of extreme anguish and maintains a rigid attitude often with the legs flexed, being afraid even to stir. The abdomen is painful to the slightest touch. Nausea and constant singultus soon appear. Sometimes the patient is greatly tormented with romiting. A few hours later, in addition to these symptoms, the area of liver dulness may be found 
absent in consequence of the escaped gas which has accumulated above its surface and has pressed it down. Dyspnoea and coma ultimately set in and the patient succumbs.

If perforation has taken place after adhesions have been formed, the same complications occur as in ulcer of the stomach under similar conditions. The duodenal nlcer often heals and there is a complete disappearance of all the morbid symptoms. Sometimes the cicatrix leads to a stricture of the duodenal lumen and then gives rise to ischochymia.

Course.-The duodenal ulcer has, as a rule, a very protracted course. In some instances a perfect cure nay be established without any ill consequences. In the majority, however, complications are common. Hemorrhages, obstruction of the duodenal lumen in consequence of the stenosis and perforation are often observed.

Diagnosis. - The diagnosis of a duodenal ulcer can be made with certainty only in a very fow instances. Most often only a probable diagnosis will be possible. A duodenal ulcer can be diagnosed with certainty if the symptoms of ulceration follow within a short period after extensive scalding of the skin has taken place. The sudden development of icterus in a case presenting symptoms of gastric ulcer speaks with a certain amount of probability for a duodenal ulcer if gall stones can be excluded. The points which indicate a probable location of the ulcer within the duodenum are the following: 1. The pains usually appear from half an hour to three hours after the ingestion of food and are situated most often to the right of the linea alba in the pyloric region. They nerer radiate to the back. 2. Repeated attacks of melrena, either not associated with hrematemesis or in which the latter was only slight compared with the melæna. 3. Most of the patients 
are men presenting a healthy appearance. 4. Perforation is a frequent occurrence in duodenal ulcer, while it is very rare in the course of gastric ulcer. If all these points are found associated, then a probable diagnosis of duodenal ulcer may be made, otherwise it is uncertain.

With regard to the differential diagnosis between ulcer of the stomach and that of the duodenum, Leube 'stated that in the latter the gastric contents show a normal degree of acidity, while in gastric ulcer, as a rule, hyperchlorhydria prevails. This point, however, is not of much value, for on the one hand cases of gastric ulcer are found with a lessened degree of secretion, and on the other hand duodenal ulcer may be attended with hyperchlorhydria. In the two cases of duodenal ulcers mentioned above which had been operated upon, the condition of the gastric juice in one was normal, while the other showed intense hy perchlorhydria. The differential diagnosis between ulcer and cancer of the duodenum is the same as that between ulcer and cancer of the stomach or pylorus.

Prognosis.-The prognosis of duodenal ulcer is almost always quite serious, as complete recovery is very rare. Relapses after apparent perfect recorery often occur. The sequelr to which the cicatrizing process may give rise, namely, obstruction of the duodenal lumen, must also be taken into consideration, and the possibility of death from perforation should never be forgotten. Another danger lies in the formation of a cancerous growth on the base of the ulcer.

Treatment.-On the whole the treatment must be conducted on the same line as that of ulcer of the stomach.

'Leube : von Ziemssen's "Handbuch der speciellen Pathologie und Therapie," Bd. vii., Abth. 2. "Die Krankheiten des Magens und Darms," Leipzig, 1876. 
In some cases the advisability of operative intervention must be considered. Cases in which a duodenal ulcer can be diagnosed with great probability and in which hemorrhages have recurred several times may perhaps be subjected to a gastro-enterostomy during the period of comparative euphoria. For by this procedure the duodenum is relieved of a great deal of irritation caused by the passage of the chyme, and the ulcer is thus given a better chance to heal. Cases in which the cicatrix has led to a partial stenosis of the duodenal lumen should certainly be operated upon, pyloroplasty or gastro-enterostomy being selected.

\section{EMBOLIC AND THROMBOTIC ULCERS.}

This group of ulcers resembles the duodenal ulcer in that disturbances of the circulation are the exciting causes. These ulcers are of very rare occurrence. Embolic ulcers were first described by Parenski.' They originate in consequence of emboli which are carried into the fine branches of the intestinal arteries, either from some abscess carity or from a focus of atheroma or endarteritis.

The pathological changes of the intestine after such an occurrence are slight if a very small vessel, a capillary or an arteriole, has been occluded. In case the embolus is of an infectious nature, infiltration and formation of pus soon develop, and the process may quickly penetrate down to the serosa and infect the peritoneal carity. It may also rapidly reach the intestinal lumen and thus produce an ulcer. In the infectious cases the fatal issue often ensues so quickly that there is hardly time for a complete formation of the ulcer. In such instances only the initial stages of the ulcerative process can be discovered. Fine nodules

'Parenski : Wiener med. Jahrbücher, 18r6, Heft 3. 
will be noticed in the intestinal wall originating from the submucosa and consisting of accumulations of round cells in the centre of which are very small blood-vessels.

The symptoms of these embolic ulcers are the same as those caused by other ulcerative processes of the intestines, namely, severe pain which may be of a colicky nature, tenderness on pressure over the abdomen, and diarrhoa with more or less bloody admixture. If these symptoms are present and embolic processes can be discovered in other organs, then the diagnosis of embolic ulcer of the intestine is probable.

The clinical symptoms and the anatomical changes resulting from the obstruction of a very small blood-vessel of the intestines are comparatively slight, compared to those which rapidly appear if the embolus has entered the arteria mesaraica superior. This affection is extremely rare; only nimeteen cases have been described in literature. The emboli which have been found in the arteria mesaraica superior itself or in its branches could be traced to the left heart or to the aorta, which was the seat of excrescences due to endocarditis or atheroma. There is either a total obstruction of the entire mesaraic artery or several larger and numerous smaller branches of this vessel are occluded. The changes which frequently result after the embolus has excluded the organ from circulation are hemorrhagic infarcts and necrosis with partial peritonitis. According to Litten, " after an occlusion of the arteria mesaraica superior or its branches, the intestine is deprived of all arterial blood, there being no vicarious blood current from any anastomoses of these vessels. The arteria mesaraica superior, although it forms anatomical anastomoses,

'Litten: "Ueber die Folgen des Verschlusses der Arteria mesaraica superior." Virchow's Arch., Bd. 63. 
acts functionally like a terminal artery. The reason of this is that the anastomosing vessels are of a very small calibre and pursue a very long course, and hence the mesenteric arteries are not able sufficiently to supply with blood the region deprived of its circulation.

The pathological changes which appear after the occlusion of this artery consist of venous hypersemia, hemorrhagic extravasations, odema, and necrosis. In that part of the mesentery and intestine which was supplied by this occluded vessel, the smaller arteries branching off from the latter are contracted and empty, while the reins of the serosa and mesentery are overfilled with blood. The mucous membrane appears dark red; the entire intestinal wall is cedematous and swollen; small hemorrhages exist all over the mucous membraue and in the mesentery; and the intestinal canal contains extravasated blood either fresh or tarry looking. If the process has lasted for some time, necrotic changes soon appear and the mucosa presents a dirty brownish-green appearance and may be wiped of from the other layers like a slimy coating. The serous layer may be the seat of inflammation not only over the involved intestinal segment, but also over other still healthy intestinal coils, the latter being agglutinated and covered with a deposit of fibrin. In the peritoneal cavity there may be a bloody fluid or a purulent exudation.

The clinical symptoms of an embolus of the superior mesenteric artery have been best described by Gerhardt ${ }^{1}$ and Kussmaul. ${ }^{2}$ They are not always alike, and two groups of cases may be easily discerned. In the one, being

'Gerhardt: "Embolie der Arteria mesenterice." Würzburger med. Zeitschr., 1863, Bd. iv.

"Kussmaul : "Zur Embolie der Arteriæ mesenterice." Würzburger med. Zeitschr, 1864, Bd. v. 
the larger, an intestinal hemorrhage is the feature most marked, in the other the affection presents the picture of intestinal occlusion with or without any signs of peritonitis. As a rule the disease sets in suddenly with violent colicky pains involving the entire abdomen or some portion of it, usually in the neighborhood of the navel. Soon the pains grow diffused and there is an extreme tenderness on pressure over the abdomen. Sometimes the pain is accompanied by romiting; in rare instances, however, the pain may be entirely absent. Such a case has been mentioned by Nothnagel. Intestinal hemorrhage, which is the chief symptom, soon occurs. As a rule sereral bloody stools appear in succession, which have a dark, almost black, brown or tarry appearance and occasionally a very fetid odor. The blood of the hemorrhage, however, is not always necessarily roided per rectum, for it may remain in the intestinal canal. The symptoms, however, which characterize a profuse intestinal hemorrhage (falling of the body temperature and collapse) will never be missing. In the second group of cases there are merely signs of an acute intestinal occlusion; pains, constipation, and peritonitis being the only symptoms.

The diagnosis of this affection can be made, according to Kussmaul and Gerhardt, in cases in which the source of the embolus can be determined. An intestinal hemorrhage occurs (for which no primary lesion exists), colicky pains of great violence and later a tympanitic swelling of the abdomen and exndations make their appearance. The diagnosis can be possibly made ouly if all the just mentioned points exist. Otherwise, especially if the intestinal hemorrhage is missing, the diagnosis cannot be made during life.

The prognosis of this affection is very grave. As a rule 
it ends fatally. It appears, howerer, according to Virchow, that in rare instances a recovery is possible after long illness, a collateral circulation haring slowly dereloped.

With regard to treatment, there is no special indication for this affection. The symptoms will have to be treated as such.

Embolus of the inferior mesaraic artery is a very rare occurrence. Two cases have been described by Hegar ' and Gerhardt. The prominent symptoms are riolent colicky pains, tenesmus, and bloody stools. The mucous membrane of the small intestine remains normal, while that of the colon, $\mathrm{S}$ romanum, and rectum becomes intensely red, succulent, and contains effusions of blood here and there. Severe anatomical lesions of the intestines, howerer, are absent, for the circulation is quite quickly re-established through anastomosis with the superior mesenteric artery and with the rectal arteries of the hy pogastric ressel.

Similar to the lesions of the embolic process of the superior mesaraic artery are the consequences which result from a thrombus within the mesenteric veins or the portal vein. A few cases of this nature have recently been observed by Pilliet, ${ }^{2}$ Grawitz, ${ }^{3}$ and Eisenlohr. ${ }^{4}$ The clinical picture of these cases is as follows: There appear suddenly violent colicky pains in the abdomen. The latter swells up and grows intensely painful on pressure. Often vomiting is present, occasionally hæmatemesis. There

' Hegar: "Embolie der Lungenarterie und der Arteria mesaraica inferlor." Virchow's Arch., Bd. 93.

2 Pilliet: "Thromboses des veines mesaraiques." Progrès méd., 1890, No. 25.

${ }^{3}$ Grawitz: "Ein Fall von Embolie der Arteria mesaraica superior." Virchow's Arch., Bd. 110.

"Eisenlohr: "Zur Thrombose der Mesenterialvenen." Jahrbücher der Hamburger Staatskrankenanstalten, 1890. 
may be coustipation or very frequent diarrhœeal and bloody morements. Accompanying these symptoms there is always collapse. The course is also a very rapid one, the fatal end appearing after two or three days. This affection is liable to occur in advanced pulmonary tuberculosis, in highly marasmic conditions like the malarial cachexia, then as a consequence of pressure of the portal vein, in cirrhosis and cancer of the liver. All abdominal neoplasms may likewise produce a thrombotic condition of the veius by pressure. The same may happen in chronic peritonitis by the formation of constricting cicatricial tissue. Similar processes also arise whenever the intestine experiences pressure or incarceration at a circumscribed spot. The venous circulation becomes obstructed by the pressure, while the arterial blood supply owing to its elastic walls remains undisturbed. In consequence of the lacking outflow of the blood, hyperæmia appears, then follow hemorrhagic infarcts, and lastly necrosis.

As the symptoms and treatment of the following classes of intestinal ulcers are identical, we shall discuss them togethcr later on, after having first given the etiological and anatomical features of each separately.

\section{AMYLOID ULCERS.}

Amyloid processes within the intestine were first described by Virchow' in 1855 . The amyloid changes start in the walls of the small blood-vessels (capillaries and the finest arteries, occasionally also the veins).

At first the vessels of the mucosa alone are affected, but afterward the process may extend through the submucosa and even through the entire intestinal wall down to the

'R. Virchow: "Ueber den Gang der amyloiden Degeneration." Virchow's Arch., Bd. 8. 
serons layer. The amyloid degeneration may also involve the muscularis mucosæ, or even the entire muscular layer of the intestinal walls. The amyloid degeneration of the blood-ressels makes them friable, thereby often leading to necrotic processes with the formation of small ulcers.

Amyloid changes are found more often in the small intestine than in the large bowel. The mucous membrane of the affected part has a waxy and pale appearance. The villi are missing here and there.

The diagnosis can be positively made by means of the characteristic color tests. A solution of iodine poured over the suspected area gives a brownish-red color which becomes violet or blue after the addition of sulphuric acid; a solution of methyl riolet produces a bright pink color.

We have reason to suspect amyloid processes within the intestine in conditions which are known to be often associated with this process, as tuberenlosis, syphilis, leukæmia. Especially is this true if amyloid degeneration is detected in other organs (spleen and liver) as shown by their enlargement, and besides there are signs of chrouic diarrhoea and insufficient intestinal absorption. There are, however, no positive means of establishing the diaguosis of amyloid degeneration of the intestine during life.

\section{TUBERCULOUS ULCERS.}

Tuberculosis of the intestines is of rery frequent occurrence. While it usually appears in phthisical patients, there are also cases of an undoubted primary intestinal tuberculosis. According to Frerichs, ${ }^{1}$ a tuberculous affection of the ileum is found in eighty per cent of the cases of chronic pulmonary phthisis. Bayle in 1810 was the first

${ }^{1}$ E. Frerichs : "Beiträge zur Lehre von der Tuberculose," Marburg. 1882. 
to observe the occurrence and frequency of tuberculous ulcers of the intestine. The seat of these ulcers is principally in the ileum, especially in its lower portion. They may extend from this point downward orer the colon to the rectum or upward over the entire ileum, jejunum, and even the duodenum.

The development of the ulcer takes place in the following way: In one of the solitary follicles a miliary tubercle forms by exteusive accumulation of cells, the latter swell up; after a time a caseous degeneration appears in the centre and the swollen follicle bursts; thus a small peasized ulcer is formed. In the same way tuberculous processes may develop in the agminated follicles and also lead to the formation of ulcers. But whereas Peyer's patches are equally affected in their entirety in typhoid fever and intestinal catarrh, in tuberculosis the infiltrations are confined only to sereral follicles of the group, while others belonging to the same patch remain intact.

The ulcar enlarges either by spreading directly at the periphery or by the coalition of several defects. As a rule the extension of ulcers into the deeper layers proceeds in a line transversely to the intestinal lumen corresponding to the direction of the ressels supplying the bowels. Thus in the small intestine the ulcer spreads in a line parallel with the valvulre conniventes, and thus may form a circular defect over the entire lumen of the intestine, transversely to its longitudinal axis (the so-called tuberculons girdle ulcer). There exist, howerer, ulcers of an oblong or entirely irregular shape. With regard to the depth of the ulcer it usually penetrates to the muscularis and remains at a standstill there. Small tuberculous foci, however, are often met with within the latter, usually connected with the lacteals. Sometimes a destruction of the mus- 
cular layers is also present and the ulcer may advance down to the serosa and may eren perforate into the peritoneal cavity.

The fully developed large tuberculous ulcer has an irregular shape, and mostly a bright red margin, being partly smooth, partly overlapping, sometimes undermined. Its base is pultaceous, consisting partly of decomposed tissue, partly of swollen remnants of the mucosa. Tuberculous infiltrations are noticeable here and there at the base as well as at the margin. The surroundings of the ulcer often show catarrhal changes. The serosa over it is usually in a state of chronic inflammation, being reddened, thickened, and surrounded with fibrinous exudations. Sometimes there are agglutinations with other intestinal coils, the omentum, or other immediately adjacent organs. The frequency of these peritonitic adhesions explains why perforations of tuberculous ulcers within the intestine are comparatively so rare.

Tuberculous ulcers very rarely show a tendency to heal, the process as a rule progressing steadily and leading to the formation of new nodules in the neighborhood of the margin. In very few instances, however, cicatrization of the ulcers takes place. The latter, when occurring in ulcers of girdle shape, may produce a stenosis of the intestinal lumen.

Tuberculous ulcers are very rarely primary, that is to say, developing in the intestines without a previous tuberculous affection existing in other organs. In most instances they are secondary and are met with in patients who are in a more or less advanced stage of pulmonary tuberculosis.

The ultimate cause of tuberculous processes in the intestine is Koch's tubercle bacillus. The latter may be carried into the intestinal caual with the sputum which 
phthisical patients swallow, or it may also, in rare instances, be ingested directly with the food. Thus, meat and milk of tuberculous cows may cause primary tuberculosis of the intestine. This condition is specially frequent in infants on account of their being fed with milk either from phthisical nurses or tuberculous cows.

\section{SYPHILITIC ULCERS.}

Syphilitic ulcers of the intestines are quite rare. In the small intestine they are mostly met with in the new-born. Here the ulcers are found either singly or in great numbers over the entire small intestine. They originate in the lymphatic apparatus of the mucosa and submucosa, first forming gummata within the intestinal walls, which afterward undergo rupture. Syphilitic ulcers of the small intestine have also been observed in adult life (Klebs,' BirchHirschfeld ${ }^{2}$ ).

Of greater clinical importance are the acquired syphilitic ulcers which often occur principally in the lower part of the colon and the rectum, including the anus (most frequently the lower part of the rectum a few centimetres above the anus is affected). We may have primary ulcers of the rectum through direct infection after a preternatural coitus. These are observed principally in men and are located in the median line of the anus. They are characterized by a hard base, sharp margins, and bacon-like appearance. We may also have secondary ulcers due to constitutional syphilis. Condylomata and gummata may undergo degenerative changes and form ulcers, which by their cicatrization very often give rise to the development of stric-

${ }^{1}$ Klebs: "Handbuch der pathologischen Anatomie." Berlin, 1868.

2 Birch-Hirschfeld : "Lehrbuch der pathologischen Anatomie, " Leipzig, 1887. 
tures of the rectum. The latter variety is much more frequently found in women than in men. Amoug two hundred and nineteen patients with constricting rectal ulcers Poelchen ${ }^{1}$ found one hundred and ninety women. This anthor, however, correctly remarks that not all these ulcers resulting in stricture are due to sy philis. In a great many instances their origin is attributable to a gonorrhoeal affection of the Bartholinian glands which ultimately through infection leads to destructive processes within the rectum. Some of these ulcers may also result from traumatic causes, such as the frequent use of clysters or hard fecal matter irritating the mucous membraue.

\section{TOXIC ULCERS.}

Under the term toxic nlcers of the intestine are understood defects which derelop in consequence of abnormal (toxic) prodncts contained in the blood. Thus intestinal ulcers occur in severe forms of nephritis, especially when they are complicated with uremic symptoms. In leukæmia and scurvy such ulcers are also met with. Intestinal ulcers arising in cases of poisoning with mercury likewise belong to this group. The ulcerative process in all these cases is best explained as due to necrosis in consequence of the altered condition of the blood.

Symptomatology.-The symptoms which accompay ulcers of the intestines vary greatly. In the following we shall enumerate all the symptoms which may be met with in these conditions.

1. Diarrhoa. Frequent loose morements are often present, especially if the ulcer is situated in the lower part of the large bowel. Ulcerations of the small intestines,

'Poelchen: "Zur Aetiologie der stricturirenden IIastdarmgeschwüre." Virchow's Arch., Bd. 12 
crecum, and the upper end of the lange bowel do not cause diarrhoea, unless there is some other complicating affection (a catarrhal condition of the bowels or an amyloid state). Bot eren if the ulcer is situated in the lower part of the colon, diarrhoea mas be absent in rare instances.

2. The cocurrence of blood or pus in the dejecta. Blood mar be roided with the stools in consequence of a small hemorrhage of the ulcerated intestive. If there is no gastric alcer, and other srmptoms point toward intestinal ulcer, the presence of blood will help to make the diagnosis more Irobable. But it is br no means a positire sign, for, on the one hand, an intestinal ulcer may exist without any hernorrhages, and, on the other hand, intestinal hemorrhages mar occur from other causes than ulcer. The iresedce of pas in the stools seems to hare much greater ininortance. According to Nothnagel, real pus (numerous roand cells) in the freces is one of the most raluable signs of alceration of the intestines. It is to be understood that yus mas also be present in ulcerative processes accompanring neoplasms of the intestines and in abscesses which oren into the intestive. The latter two conditions will have to be excluded before re can infer the existence of an intestinal ulcer from this symptom. The awount of pus in trae alcerations of the intestines is, as a rule, rery small, aud it is Decessary to esamine the dejecta quite thoroughls in order to find it. While the presence of pus is so important a srmptom in intestinal nlcer, its absence br no means speaks agrainst it. For there may be no formation of yus at the site of the ulceratire spot, or the pus roar be changed to such a degree that it is no longer recognizable, especiall! if the ulcer is situated high up in the intestine.

3. The existence of tubercle bacilli in the dejecta is of 
great importance in cases in which pulmonary tuberculosis can be excluded, since they then show primar! intestinal tuberculosis. The absence of the tabercle bacilli does not speak against the presence of ulceratire areas in the intestines, nor does their presence pasitirelr indicate a tuberculous affection of the intestine when pulmonary tuberculosis exists, for these microbes are then usualli derired from the sputa which hare been swallomed and carried dorn with the passages.

4. Prins. If pains exist in the abdomen in a more or less circumscribed spot for a long period of time, and if these pains are increased on pressure, thes are probably due to an alcer in the intestines. The absence of this srmptom, howerer, speaks in no mar agrainst an ulcer, nor is its presence an absolute positive șrmptom for ulcer.

The general state of the șstem need not be disturbed, if the nlcers are only fem in number and rery small. If their number, howerer, is great and their size extensive, so that a large part of the intestinal tract is inrolred in the ulcerative process, then nutritire disturbances will manifest themselves and marked emaciation take place.

Diagnosis. - As mas be seen from the description of the srmptoms, the diagnosis of ulcer of the intestines is, as a rule, quite difficult. Their existewce war be suspected whenever there is diarrhcea of a serene nature and more or less intense pain orer a certain fired region of the abdowen extending orer a great period of time. A positive diagnosis can be made onls in the following instances:

1. If necrotic pieces of the intestinal mucosa or pas appear in the stools (in the latter instance the perforation of an abscess into the intestine has to be erclnded).

2. The more or less frequent appearance of small amounts 
of blood quite changed in the stool, if ulcer of the stomach or vicarious bleeding cau be excluded.

3. Diarrhoea and the constant appearance of tubercle bacilli in the stools, when pulmonary tuberculosis can be excluded. This points to the presence of tuberculous processes (ulcers) in the intestine.

4. If the ulcers are situated in the lower part of the colon or rectum and are accessible to a direct visual examination.

the nature of the ulcers (whether catarrhal, tuberculous, syphilitic, or toxic) must be elncidated by a thorough knowledge of the history of the case and the results of an accurate examination of the patient.

Prognosis. - The prognosis of intestinal ulcers will depend largely upon their number, size, and nature. A few small catarrhal ulcers will heal quickly without any further trouble. Amyloid ulcers hardly ever show a tendency to heal. Tuberculous ulcerations occasionally are amenable to treatment, still more so are the syphilitic ulcers. Very extensive ulcerations, no matter of what nature, are very dangerous to life.

Treatment.-In the treatment of intestinal ulcers the etiological factors play the greatest part. Thus, in tuberculous ulcers general hygienic rules will have to be observed. An out-of-door mode of living, and, if possible, in the mountains, should be recommended. Guaiacol carbonate, creosote, ichthalbin are of value. In syphilitic ulcers general anti-syphilitic treatment should be instituted: inunctions with mercury, or iujections of sublimate or calomel, or the administration of large doses of potassium iodide. In toxic ulcers (as those due to uræmia and mercurial poisoning) the treatment must be directed against the primary trouble. Besides the etiological therapy, intestinal ulcers require specific and 
symptomatic treatment. The treatment directed to the healing of the ulcers is very successful if the latter are situated in the rectum or in the lower part of the colon, while this object can hardly be attained if they exist high up in the colon or in the small intestine. In the former instance the ulcers, if accessible to view, may be directly treated by the application of a strong solution of nitrate of silver or protargol. If not visible but situated in the colon, injections of a 0.2 to 1 per cent. solution of nitrate of silver or of tannic acid of the same strength into the bowels are of value. If the ulcers are situated in the small intestine, large doses of subnitrate of bismuth ( 1 to $2 \mathrm{gm}$. [gr. xv. to $\mathrm{xxx}$.] three times a day) may be tried. The symptoms which accompany the ulcer and vary from time to time will have to be treated as such. Diarrhoea, hemorrhage, and pain must be combated with the customary remedies.

Most patients should be kept abed for some time. The application of a hot-water bag or a wet pack over the abdomen is very beneficial.

The diet should contain nourishing but easily digestible and non-irritating food. Thus, milk, kumyss, matzoon, eggs beaten up in milk, soft-boiled eggs, farina, oat meal cooked in milk, mutton broth, chicken soup, scraped beef, calf's brain, sweetbreads, cacao, tea, and toast may be given. 


\section{CHAPTER VI. \\ NEOPLASMS OF THE INTESTINE.}

MALIGNANT GROWTHS.

\section{Cancer.}

Definition.-An epithelial neoplasm of the intestinal walls.

Etiology.-The etiology of intestinal cancer, like that of cancerous disease of other organs, is still unknown. The traumatic theory (repeated irritation of one particular area) appears quite plausible with reference to this organ. As will be seen later, this malady occurs much more frequently in those parts of the bowels in which the passage of fecal matter is more apt to be retarded, and in consequence to cause irritation.

With regard to sex, it is generally accepted that intestinal cancer occurs somewhat oftener in men than in women. With reference to age it is chiefly met with during the period from forty to sixty-five years. Cancer of the intestine is occasionally found also in young people, this happening much more commonly than cancer of the stomach or of other organs. Nothnagel ' has observed cancer of the crecum in a twelve-year-old boy, and Schoening ${ }^{2}$ reports two cases of rectal cancer in girls seventeen years old.

${ }^{1}$ H. Nothnagel: "Die Erkrankungen des Darms und des Perito. neum," Wien, 1898.

'Schoening: Deutsche Zeitschr. f. Chirurgie, Bd. xxii., 1885. 
According to Maydl, ${ }^{1}$ the total number of intestinal cancers occurring from the first to the thirtieth year amounts to oue-seventh of the entire number of cases.

Location.-With regard to location the frequency of the affection in the different portions of the bowel varies. The frequency gradually increases the lower down the growth is situated, beginning with the jejunum and ending with the rectum. Among one hundred and sixty autopsies on cases of cancer of the different organs, Maydl found in one hundred cancerous disease of the bowels. In one hundred and ten autopsies of patients suffering from intestinal cancer, Bryant ${ }^{2}$ found the neoplasm located six times within the small intestine, seven times in the crecal and ileocæcal regions, nineteen times in the transverse colon, including the hepatic and splenic flexures, seventy-eight times in the sigmoid flexure and rectum. Maydl gives the following locations of the tumor in one hundred autopsies: Two in the duodenum, four in the ileum (none in the jejunum), forty-six in the large bowel (in the rermiform process, one; cæcum, nine; ascending colon, six; colon seventeen; sigmoid flexure, thirteen), and forty-eight in the rectum. As regards cases observed during life, Maydl gives the following figures: During twelve years there were in the Wiener Allgemeines Krankenhans 246,827 patients. Among these there were 6,287 patients with cancer. Among the latter there were 254 cases of cancer of the bowels, and in 224 of these the neoplasm was in the rectum. This certainly shows the great predilection of intestinal cancer for the rectum.

Intestinal cancers are almost always primary. It is exceptional for cancer of the bowels to derelop by way of metastasis. It is obvious, howerer, that cancer in this

1 Maydl : "Ueber den Darmkrebs," Wien, 1883.

Joseph D. Bryant: Anuals of Surgery, February, 1893. 
region may develop secondarily as a result of direct extension of the cancerous process from a contiguous organ. This often occurs in cancer of the stomach, gall bladder, or pancreas. Intestinal cancer often gives rise to metastases in other organs. According to Mïller, ${ }^{1}$ these are more frequently met with in cancer of the small intestine than in that of the large bowel. The lymphatic glands are also often secondarily affected. Those in the neighborhood of the neoplasm show a greater tendency to become cancerous than those farther off.

Morbill Anctomy. - All varieties of cancer are found in the intestines. Most frequently, however, the cylindrical epithelial-celled carcinoma, having a glandular structure (adeno-carcinoma), is encountered. The latter takes its origin in the epithelial cells of the follicles of Lieberkuehn. Colloid carcinoma is quite often found in the rectum, while melano-carcinoma is here quite rare. Occasionally the pavement-celled carcinoma (epithelioma cancroid) is met with, especially in the lower part of the rectum, starting principally from the anus. It often involves the perineum and the vagina.

The neoplasm varies in cousistency according as connective tissue or cells predominate. If the former is the principal element, then the tumor presents a hard consistency (as hard as cartilage) and is termed scirrhus. In case the latter are more abundant, then it is less firm, occasionally soft and succulent. The colloid cancer as a rule contains a brownish, somewhat viscid fluid. The scirrhus shows a greater tendency toward partial necrosis in its central part. It often forms a carcinomatous ulcer.

The primary intestinal cancer frequently shows a ten-

2 Max Maller : "Beiträge zur Kenntniss der Metastasenbildung maligner Tumoren." Inaugural-Dissertation, Bern, 1892. 
dency to extend in a circular direction perpendicularly to the lumen of the bowel. Stenosis of the intestinal canal is very often the result of this circumstance. In case the stricture is of marked degree, the intestine above the strictured spot becomes greatly distended through stagnating fecal matter and gas. The bowels working hard to overcome the obstacle show thickened walls due to hypertrophy of the muscles. The irritating and stagnating contents in the dilated part of the intestine give rise to catarrhal inflammation and also to ulcers. If the stenosis has become still more pronounced, the dilatation of the intestine above it may be so excessive that a rupture of its walls ultimately occurs. Below the stricture the intestinal wall appears thinner, and if the stricture is so narrow that no contents pass downward, it appears empty and contracted. Occasionally the neoplasm constricting the intestinal lumen begins to break down and ulcerate, and this partly removes the occlusion of the intestinal canal. This, however, does not last long, for as a rule the cancer shows a tendency to grow again and to fill up the defect. Thus the free lumen of the bowel is very soon again occluded.

This partial necrotic process will also often cause more or less hemorrhage through erosion of the smaller bloodvessels. In case a larger artery or vein opens, a severe hemorrhage with fatal issue may result.

Cancer of the bowel often involves, besides the mucosa and submucosa, the muscularis and even the serosa. In the latter event perforation occurs in rare instances before adhesions have had time to form, and may result in fatal general peritonitis. In most instances, however, adhesions have formed around the involved area, and thus the perforation causes merely a circumscribed peritonitis. Even without the occurrence of perforation the cancer may 
progress from the serous layer to the peritoneum and lead to a carcinomatous peritonitis, which is often accompanied by a hemorrhagic exudation. Another series of grave complications is caused by the extension of the cancerous process to a neighboring organ which has previously become agglutinated to the bowel. The process of disintegration in the cancerous grow th then often establishes an abnormal communication between the bowel and other organs. Thus fistulous openings may occur between colon and stomach, between rectum and bladder, between rectum and vagina, between rectum and uterus, between large and small bowels, or a direct fistulous opening may form from the bowel through the abdominal wall.

Symptomatology.-Cancer of the bowel develops quite slowly and insidiously, and in most instances at the beginning gives rise to hardly any symptoms at all. For this reason it can never be detected at this time; later, however, general and local symptoms manifest themselves. While the general symptoms are common to all cancers of the small and large bowels, the local symptoms will differ according to the location of the tumor, and it will therefore be necessary to consider the different portions of the intestinal tract separately.

A. General Symptoms. - The general symptoms of cancer of the bowel are those found in maliguant growths of other organs. Of these anæmia and cachexia are the most important. Usually both are present at the same time. Sometimes one is more pronounced than the other. In some instances a general weakness, pallor, and emaciation are the first indications of a severe affection. There may be as yet no local symptoms whatever or a very slight degree of constipation and scarcely noticeable sensation of discomfort in the abdomen. Loss of appetite and slight dyspeptic sy mp- 
toms are often encountered. Fever is occasionally met with, which is due to a suppurative process and absorption of pyogenic matter into the blood. The neoplasm often gives rise to disturbances in neighboring organs by constricting or dragging upon them. Thus radiating pains from compression of nerves may arise and in the same manner disturbances of circulation. Edema of the lower extremities is often encountered, which after lasting for weeks and months may occasionally disappear shortly before death.

Sy mptoms of chronic intestinal obstruction are often present. They develop either gradually, the constipation increasing more and more, or they may appear more abruptly . The bowels, while formerly more or less regular, suddenly cease to move, and even strong cathartics are of no avail.

The clinical features of cancerous obstruction of the bowel are not different from stenosis of the intestine caused by other processes, which are described in Chapter IX. Such a sudden attack of obstruction of the bowel may terminate fatally in a few days; sometimes, however, after a total occlusion of the bowels, life continues much longer. Thus fecal retention of forty-four days' duration, without even fecal vomiting, is mentioned by Heusgen,' and another case of eighty-eight day's' duration has been reported by Cooper-Forster." Diarrhoea is frequently present in cancer of the bowels. This often serves partly to overcome the beginning obstruction of the intestinal lumen. In some cases diarrhoea alternates with constipation. In the latter instance the stools often bear signs of having passed a strictured spot. They may appear in the shape of a tape or in the form of small, hard balls. These characteristics of the evacuation are, however, by no means a positive

${ }^{1}$ Heusgen : Deutsche med. Wochenschr., 18\%.

2 Cooper-Forster: Medical Times and Gazette, September, $186 \%$. 
proof of a real stricture, for they are also met with in merely neurotic conditious. The stools often contain an admixture of mucus, blood, or pus. In case the progress of the necrosis of the neoplasm is pronounced, the stools during that period have a very offensive, almost unbearable odor. In rare instances particles of tumor may be discovered in the dejecta, which show under the microscope the exact nature of the neoplasm. If these particles are of a large size (cherry or walnut) they will be easily discovered in the stools; but if they are minute, a thorough examination of the fecal matter will be necessary in order to find them. Washing out of the bowels will often be helpful to discover such minute pieces of the growth, in case the latter is situated in the colon.

While all of the above symptoms are of great value, they are unimportant compared with the physical signs of a tumor. Its presence in a doubtful case in most instances helps to clear up the diagncsis. The tumor is often easily palpable and bears the general characteristics of a cancerous growth. It is hard and presents an uneven nodular surface. Its size varies greatly, being often that of a walnut and occasionally that of an apple or still larger. In the latter instance the mere inspection of the abdomen may already show the presence of the tumor. In autopsies the neoplasm is frequently found much smaller than it appeared to be during life. The cause of this is the hypertrophy which occurs in the walls of the bowel above the tumor, together with the accumulation of fecal matter at the same place. The tumor is usually situated in the lower half of the abdomen, principally in the left iliac region, not only because this part of the intestine is so often affected, but also because a neoplasm of other parts of the bowel, if not fixed by adhesions, is as a rule dragged down 
by its own weight into this region. Intestinal neoplasms as a rule show a high degree of mobility. Often they can be moved with the hand in all directions in the abdominal cavity. The only exceptions to this rule are tumors of the duodenum, the sigmoid flexure, and the crecum, which are more or less fixed.

With regard to the detection of the tumor a thorough palpation of the abdomen (if the abdominal walls are very rigid, under ether or chloroform narcosis) is necessary. A digital examination of the rectum, and, in women, of both rectum and vagina, will in most instances be required. A bimanual examination will also be found useful. In case the affected area in the rectum is not accessible to digital examination, inspection of this organ and in some instances a manual examination under anrsthesia with the whole hand must be resorted to.

When the disease is fully developed, peritonitis (either circumscribed or general) often appears as a complication. It may be simply caused by the inflammatory processes accompanying the neoplasm or be of a real cancerous nature. While at first it is impossible to differentiate these two conditions, later on it is as a rule not difficult to determine which of the two is present. The discovery of a hemorrhagic exudation and of a few nodules under the abdominal wall will indicate that a caucerous affection of the peritoneum is present. An acute perforation peritonitis is much more rareand leads to shock and sudden death, or in the presence of adhesions to grave complications in consequence of fecal abscesses. If the perforation occurs into adherent neighboring organs, new communications may be formed between them and the intestine; they aggravate the condition and are of great clinical importance. The following communications are frequently met with: 
1. Fistula betueen stomach and colon. The fistulous opening may freely communicate with both cavities or only in one direction on account of the formation of a valve. If the passage has the direction from the stomach into the colon, symptoms of lientery develop, and undigested and uuchanged foods, as for instance pieces of meat, potatoes, spinach, and the like, appear in more or less large quantities in the stools; often diarrhœea manifests itself shortly after a meal and examiuation of the evacuation shows numerous particles of food from the last meal. Lavage of the stomach performed in such a case will often show that the liquid has escaped from the stomach in considerable quantity and may occasionally be voided by the rectum. The admixture of some coloring matter to the water used for lavage will facilitate the recognition of this condition. If the communication has a direction in the opposite way, namely, from the colon into the stomach, there will be an appearance of fecal matter in the latter. In that event the gastric contents always contain decomposed and fetid material, and romiting of fecal matter is frequently the result. Inflating the colon with air will often cause a filling up of the stomach with this gas, and again irrigation of the bowel with water (either clear or stained) will be followed by its appearance in the stomach, which may be easily discorered by introducing a tube into this organ and evacuating the gastric contents. If the fistulous opening has a free communication in both directions, then symptoms of lientery and fecal vomiting may be present at the same time or they may appear alternately.

2. In case of a communication between rectum and bladdex, small particles of fecal matter and gas appear in the latter organ and may be roided through the urethra. They give rise to a putrid crstitis. Occasionally urine may pass 
from the bladder into the rectum and be discharged with the stools. The recognition of the latter condition is, however, more difficult.

3. Communications between the rectum and uterus or vagina are also met with and give rise to the passage of fecal matter through these organs.

4. A fistulous opening may exist between the bovel and the abdominal wall. This fistula may discharge esternally a putrid secretion having a fetid odor and containing particles of fecal matter or chyle, depending upon its location, whether in the large or small intestine.

All these fistulous communications appear as a rule in the last stages of the disease. They are, howerer, by no means characteristic of caucer of the intestive, for they may also, but very rarely, derelop in consequence of other ulcerative processes in the borrel (tubercles). Again they may be a result of a cancerous growth in the stomach inrolving secondarily the intestinal tract.

The urine does not show anything characteristic of caucer. Howerer, it often coutains large amounts of indican; acetone and diacetic acid have also been occasionally met with.

B. Symptoms Due to the Location of the Neoplasm.-(a) Cancer of the duodenum. In the duodenum the neoplasm almost always causes gastric symptoms similar in uature to those of cancer of the pylorus. Thus anorexia, pains, romiting, and dilatation of the stomach will be the predominating features. If the tumor is situated near the pylorus in the superior horizontal portion of the duodenum it will be quite morable, and a differential diaguosis between cancer of the pylorus and that of the beginning of the duodenum will hardly ever be possible during life. In case the neoplasm is situated in the descending part of the 
duodenum, in the immediate neighborhood of Vater's papilla, icterus is often encountered. In such cases the initial symptoms may be jaundice and sometimes chills. The icterus may remain stationary or vary in intensity from time to time according to the degree of the obstruction of the dnet caused by the neoplasm. Ulceration of the tumor may for a while open a passage for the bile and the jaundice'may then temporarily disappear. If the cancer is located below Vater's papilla, especially in the inferior horizontal part, the gastric contents will frequently show the presence of a large amount of bile. In the latter two instances the tumor, if accessible to palpation, is not movable. On acoount of its deep situation it can frequently not be discorered.

(b) Cancer of the small intestine. According to the regiou in which the neoplasm is situated, whether at the beginning of the jejunum or in the lower parts of the ileum, gastric or intestinal symptoms will-predominate. There may be anorexia and vomiting, or, on the other hand, good appetite and apparently good stomach digestion, but obstinate constipation. The tumor is often accessible to palpation, and is as a rule very morable.

(c) Cancer of the large bowel. Pains are frequently encountered at a localized spot in the region of the large bowel. They may exist before a tumor can be palpated and may be felt either in its immediate neighborhood or in almost exactly opposite portions of the colon. Thus cancer of the crecum may give rise to pain in the sigmoid flexure, and vice versa. These pains are rarely severe; as a rule they consist merely in a sensation of discomfort or in a feeling of tension. Besides these uncomfortable sensations of a more or less permanent nature, there may be more or less frequent attacks of colic. In the latter in- 
stance there may be violent excruciating pains in the abdomen, which may be relieved after passing of flatus or after a diarrhceal movement. The attacks of colic are frequently caused by the commencing obstruction of the intestine, and therefore become gradually aggravated in nature. They may lead at last to a total obstruction and be the immediate cause of death. Constipation is one of the foremost symptoms of a neoplasm of the large bowel. It is encountered in the great majority of cases; in some instauces it forms the first symptoms of the disease; at first it may be slight in nature, but becomes steadily more obstinate. Ten or twenty days may pass without a spontaneous evacuation, and eren cathartics are very slow in their action. The coustipation as a rule is accompanied by the usual symptoms resulting from it, tension and fulness in the abdomen, poor appetite, occasionally pains. The constipation may at times disappear and give place to a period of diarrhœa. In some instances diarrhoal evacuations may exist for many weeks, and they may be the predominating feature of the disease.

(d) Cancer of the rectum. The symptoms met with in cancer of the rectum resemble more or less those of a neoplasm of the upper portion of the large bowel. Here, however, the diagnosis can be made with greater ease and certainty. In most instances rectal cancer can be discorered by a digital examination of the rectum. By means of the latter we may discover a mass lying right beneath the mucous membrane of the rectum, over which the mucosa can be slightly moved or not at all if it is adherent. 'The surface may feel uneren and somewhat hard. Sometimes the finger encounters a constriction through which it cannot easily pass; the tissues here present the same characteristics as just described. Occasionally an ulcerated area 11 
can he discovered on the surface of the neoplasm. In cancer of the rectum situated high up (not accessible to digital examination), several clinicians have advised examination with the whole hand passed through the rectum. This, however, can be done only under chloroform narcosis and is not free from danger. Such an examination may in rare instances cause rupture of the intestinal wall as stated by Volkmann.' Inspection of the recium by means of Kelly's speculum cau be easily performed and aids us in discovering a neoplasm situated quite high up in the rectum, even if not accessible to digital examination. The latter instrument may also be used in neoplasms of the lowest part of the bowel, although its use here is not of much importance, as the palpating finger gives us enough certainty in making the diagnosis.

Cancer of the rectum is as a rule accompanied by severer pains than that of the large bowel. These as a rule are local in character. They often radiate toward the crecum and the lower lumbar region, toward the bladder and genital organs, and sometimes in the direction of the sciatic nerves. In case the neoplasm involves the anus, there is an exacerbation of the pain at each evacuation. Tenesmus is constantly present in the latter iustance. If such a neoplasm of the lower parts of the rectum becomes nlcerated, the tortures of the aftlicted person can hardly be described. The patient as a rule is afraid of having an evacuation, and tries to keep it back as long as possible. At last there is a movement containing fecal matter, mucus, blood, and sometimes pus, under most excruciating pains.

Leube has directed attention to the fact that hemorrhoids are frequently associated with the neoplasm of the

'Volkmann : "Ueber den Mastdarmkrebs." Volkmann's Sammlung klin. Vortraege, No. 131. 
rectum. This is of importance, as it shows that the presence of hemorrhoids should not lead one to abstain from digital rectal examination. If a patient has complained of constipation for a short period (a few months) and hemorrhoids have developed during this time, the latter are rather indicative of a more serious condition, and a digital examination of the rectum should always be undertaken under such circumstances.

Course.-An uncomplicated intestinal cancer may last for years. Frequently, however, the time is much shorter. Many complications are liable to occur-hemorrhages, perforation peritonitis, rupture of the intestines, ileus, autointoxication, extension of the cancer to other organs, and metastases. On account of these many possibilities the life of the patient may be shortened, and it is hardly possible to foresee its duration. In some instances a condition of coma (coma carcinomatosum) appears quite early. It is generally assumed that the latter is due to auto-intoxication, either by the products of decomposition of the intestinal contents or by the toxins of the cancer. Ewald in such a case succeeded in isolating a body from the urine belonging to the group of diamins. In cancer of the duodenum the general nutrition suffers very early and extensively, and for this reason the duration of life is short. In cancer of the rectum nutrition is well maintained for a long period, and for this reason the duration of life in the absence of complications is quite long (about four years). In case anæmia of a high degree supervenes, a marasmic thrombosis may develop and the patient may die in consequence of an embolus of the lungs. If intestinal cancer is unattended with complications, death often results in consequence of general exhaustion.

Diagnosis.-The diagnosis of intestinal cancer can be 
made with certainty in the following instances: 1 . If by abdominal or rectal palpation a tumor can be detected which is situated in the small or large bowel, and accompanied by symptoms of cachexia and disturbances of defecation. 2. The presence of a tumor as just described, and the discovery of small particles of the neoplasm in the evacuation giving microscopically the appearance of a cancerous growth. 3. Gradually increasing disturbances of the bowel for a few months in a heretofore healthy person, accompanied by cachexia and symptoms of a beginning or already developed stricture of the bowels and the presence of a small particle of growth in the stools, giving as above microscopically the picture of cancer.

If there is no tumor and if nothing cancerous is found in the stools, the diagnosis can never be made with certainty. A probable diagnosis of intestinal cancer will have to be made if cachexia is present, together with symptoms of gradually developing intestinal disturbances, indicating the beginning of an obstruction of the bowel, in a middle-aged or elderly person who has been well up to a few montlis before.

Prognosis. - The prognosis of intestinal cancer is always nnfarorable. Unless an early operation and total excision of the growth is resorted to, a fatal issue is sure to follow, althongh the exact duration of life can hardly be predicted, the latter depending upon subsequent complications.

Treatment.-A cure is possible only by a total and thorough removal of the growth. We must therefore always endeavor to make the diagnosis as early as possible and advise an immediate operation whenever feasible. Cancer of the rectum can be recognized quite early and resection of the neoplasm is here followed by brilliant results. If 
the tumor is located farther up in the large bowel or in the small intestine, then the results of an operation are not so promising, for here the recognition of the growth is possible only at an advanced period, and by that time often adhesions with other organs and cancerous infection of the glauds have already taken place. Excision of the tumor and resection of the intestine in the neighborhood of the neoplasm with an end-to-end anastomosis should be practised whenever feasible. In case, however, total resection is impossible, an entero-enterostomy or entero-colostomy, or if the cancer is situated in the rectum, a colostomy (artificial anus) will be of benefit. These operations are palliative in nature and prolong life, at the same time making it more comfortable. They are intended to allay the simptoms of obstruction and to carry the fecal matter over a new route, not passing through and thus not irritating the caucerous area. In some instances of inoperable cancer of the rectum curettage followed by the application of the thermo-cautery is of benefit for a short period.

Aside from these surgical means the treatment should be symptomatic. The diet should consist of foods containing plenty of nourishment but very little indigestible residue, thus forming only a small quantity of fecal matter. If there is stagnation of the intestinal contents, cathartics will have to be given in order to liquefy the fecal matter. This can be done by means of castor oil, rhubarb, magnesium sulphate, and so ou. If the neoplasm is located in the large bowel, irrigations with warm oil or water are preferable. The pains should be allayied by means of warm baths and cataplasms, but if these fail, by narcotic remedies, such as morphine, opium, codeine, or belladonna; suppositories being here most suitable. Eventual complications should be treated as such. 


\section{Sarcoma and Lympho-Sarcoma.}

Sarcoma of the intestine is a much rarer affection than cancer. According to Kundrat, ' in the Wiener Allgemeine Krankenhans between the years 1882 to 1893 there were 2,125 autopsies on cases of cancer. Of this number 243 were cancers of the intestives. In the same period of time there were 274 necropsies on patients with sarcoma, of which 3 were located in the intestines. Amoug 61 lymphosarcomata 9 were in the intestives. On the whole the symptomatology of these malignant neoplasms coincides with that of cancer of the intestines. There are, however, a few points in which they differ from intestinal cancer: While carcinoma is most frequently found in the lower portions of the large bowel, sarcoma shows a greater predilection for the small intestine and the upper portion of the large bowel. Thus, according to Nothnagel, among 9 cases of sarcoma of the intestiues 1 was located in the duodenum, 3 in the jejunum, 3 in the ileum, and 2 in the crcum.

Sarcoma of the intestines shows very rapid progress, and metastases in other organs are very early found. The duration of life is much shorter than in cancer, being in most cases about but nine months. There is only one instance mentioned in literature in which a patient lived one and three-quarter years after the first appearance of symptoms. Symptoms of obstruction which are so frequently found in cancer of the intestives are very rarely if ever met with in sareoma. The tumor as a rule extends over a large part of the intestines, but does not occlude the canal. Cachexia and anæmia belopg to the early symptoms, and are much more pronounced than in cancer. The progno-

' Kundrat : Gerhardt's "Handbuch der Kinderkrankheiten," Bd. iv., 2te Abtheilung, Tübingen, 1880. 
sis of this form of tumor of the intestines is absolutely fatal. Even in cases in which an operation is performed quite early, it is as a rule not of much benefit on account of the numerous metastases which derelop so early; and on this account Madelung ${ }^{1}$ even hesitates to advise surgical interference.

\section{BENIGN TUMORS OF THE INTESTINE.}

Of the benignant neoplasms the following forms are occasionally met with in the intestine: adenoma, fibro. ma, lipoma, myoma, angioma, and cyst. These growths are termed polypi if they have a pedicle. Occasionally they have a large base and form only a small prominence over the surface. The polypi are usually of small size, that of a cherry or plum; rarely they are larger, pear-sized or greater still. As a rule they are covered with normal mucous membrane. Although they are found almost everywhere in the intestinal tract, they occur most frequently in the rectum (according to Rosenheim in eighty per cent).

Among the benign tumors the adenomata are most frequently met with. They arise from the mucosa, have a typical acinous structure, and are attached to the mucons membrane either by a broad base or by a pedicle. In the latter instance they form poly poid excrescences which may cover long distances of the intestinal canal, existing in large numbers. Ewald refers to a specimen in his possession in which the inner wall of the colon was covered from the splenic curvature to the sigmoid flexure with such numerous polypi that they projected from the mucous membrane like tassels from a ribbon. The whole specimen looked somewhat like a gigantic bunch of grapes. The polypi are most often met with in children from the fourth to the ${ }^{1}$ Madelung: Centralbl. f. Chirurgie, 1892, No. 30. 
seventh year, although they also occur in grown-up persons.

With regard to symptoms the benign tumors located in the upper parts of the intestinal tract cause hardly any disturbances at all. Sometimes, however, especially if they are present in larger numbers, they may give rise to hemorrhages and catarrhal affections. On account of their small size and soft consistency it is almost impossible to discover them by palpation through the abdominal wall. In very rare instances they may give rise to serious symptoms by occluding the intestinal lumen or by causing invagination. The benign tumors located in the rectum more frequently give rise to disturbances. Thus tenesmus and difficult defecation are often met with; hemorrhages also occur frequently. Sometimes such a polypus, if situated near the anus, may protrude through this opening during defecation and give rise to severe pains. Occasionally a polypus is torn off from the intestinal wall and passed with the stools. In such an event the symptoms, if there have been any, suddenly disappear.

Whenever these tumors are situated in the lower rectum they are accessible to direct examination and treatment. The latter consists in removing them by galvanocautery or by direct surgical measures. 


\section{CHAPTER VII.}

\section{HEMORRHOIDS.}

Synonyms: Phlebectasia hemorrhoidalis. Piles.

Definition.-Diffuse or circumscribed varicose dilatations of the hemorrhoidal veins sitnated either in the subcutaneous tissue of the external surface of the anus or in the submucous tissue of the lower portion of the rectum.

Etiology. - The affection under consideration is quite frequently met with. It occurs more often in men than in women and very rarely in children. While in olden times it was believed that hemorrhoids were due to a faulty state of the general circulation or dyscrasia, it is now generally accepted that they are the result of merely local disturbances. The development of hemorrhoidal varices takes place in the same manner as that of varices of other regions of the body, principally by mechanical influences. The reason why these phlebectases are formed so often in the hemorrhoidal plexus is as follows: 1 . The hemorrhoidal veins occupy a low position of the body, no matter whether in the standing or in the recumbent posture. 2. Ther are often unduly compressed by the contraction of the muscles situated in the lower end of the rectum and by fecal masses accumulated here. The circulation is thus at certain times obstructed or altogether arrested. 3. The hemorrhoidal veins are not provided with valves, and thus blood which has passed through them can be easily forced back. 4 . The rectal veins are the remotest branches of the portal 
vein in which there is norinally but a low degree of pressure, and in which circulation is easily retarded by disturbances of the liver. As all these factors exist even uncler normal conditions it is readily conceivable that phlebectases are found in the majority of people; usually, however, they do not reach a marked development, and for this reason do not give rise to complaints.

All conditions which tend to produce lasting hy peræmia of the lower portion of the rectum give rise to the derelopment of hemorrhoids. Too prolonged sedentary or standing occupations predispose to them. In this way hemorrhoids occur in clerks, students, some artisans, for instance, shoemakers, tailors, and cavalrymen, seamstresses and washwomen, etc. High livers and people who are used. to strongly seasoned or fatty foods also often suffer from piles on account of the great fuluess of the portal circulation under these conditions. Habitual constipation also favors their development. The use of strong cathartics like aloes, colocynth, gamboge, etc., irritates the large bowel in a marked degree and often gives rise to hemorrhoids. Diseases of the uterus which lead to an enlargement of this organ and also pregnancy are predisposing causes. In a similar way affections of the prostate and tumors of the bladder as well as of other organs situated in the small pelvis often produce hemorrhoids. All diseases of the liver which are accompanied by a congestive state of the portal circulation exert a direct influence upon their formation. Diseases of the heart and luugs frequently cause congestion of the inferior rena cava and indirectly also of the rectal reins, thus predisposing to the affection under consideration.

Hemorrhoids are most frequently developed between the ages of thirty and fifty years. They are extremely rare 
in infants and children. With regard to frequency the male sex seems to be more often aftlicted than the female. Some races show a special predisposition to this disease, depending most probably upon their mode of living and their diet. Whether heredity plays a part in the development of hemorrhoids is yet unsettled.

Morbid Anatomy. - In some instances the hemorrhoidal veins are evenly dilated and can be noticed as bluish-red and tortuous vessels encircling the external anal opening. At the same time there may be no special varicose swellings; more frequently, however, besides the general congested condition of the veins there are isolated varicose protrusions which may range in size from a pea to a walnut. They vary greatly in shape: sometimes they are round, sometimes flat, sometimes again irregular. Their size greatly changes from time to time in the same person. After defecation as a rule they grow smaller. Internal hemorrhoids appear as soft nodules of a bluish hue and have thin walls. They often develop to a considerable size and make defecation difficult. As a rule, hemorrhoids occur as multiple nodules, which may cover the mucous membrane at different places, or they may encircle the external surface of the anus, or be situated above the internal sphincter. Internal and external hemorrhoids may also be present at the same time. Thus Cruveilhier 'described a case in which there existed a wreath of external hemorrhoids around the anal opening, another abore the internal sphincter, and a third a few centimetres farther up.

External hemorrhoids are at first covered with normal epidermis which can be moved orer them. Later, however, through inflammatory processes the cutis becomes adherent to the varicose nodule. At the same time the

'Cruveilhier: "Traité d'anatomie pathologique générale, " 1849. 
skin covering the nodule grows thinner through the steady pressure to which it is subjected, and it may reach a point when it breaks open after a forced defecation. The same remarks also apply to internal hemorrhoids in which the cutaneous covering of external hemorrhoids is represented by the mucous membrane of the bowel. This also becomes adherent, thinned, and may ultimately rupture.

Internal piles are best divided, according to Allingham. ${ }^{1}$ into the three following varieties:

1. Capillary Piles. These present small, florid, raspberry-looking tumors or rather vascular areas upon the mucous membrane, having a granular spongy surface and bleeding on the slightest touch; they are often situated rather ligh in the bowel; in structure they consist almost entirely of hypertrophic capillary vessels and spongy connective tissue. They resemble arterial nævi very closely, indeed, in their microscopical structure, except that they are covered externally by a very much thinner membrane and consequently are readily made to bleed.

2. Aiterial Piles. These appear as tumors varying in size, sessile or somewhat pedunculated, attaining sometimes very considerable dinnensions, glisteuing or slightly villous on their surface, slippery to the touch, hard and vascular with an artery often as large as the radial entering their upper part. When they are villous on their surface, they bleed very freely and for some reason or other have formed and grown very rapidly. On dissecting one of these tumors one will find that it consists of numerous arteries and veins frequently anastomosing, tortuons, and sometimes dilated into pouches, and of a stroma of cell growth and connective tissue, the latter most abundant.

I William Allingham and Herbert W. Allingham: "The Diagnosis and Treatment of Diseases of the Rectum." London, 1896, p. 113. 
3. Venous Piles. In these the venous system predominates. The tumors are often very large and are sometimes the size of a hen's egg. They are bluish or livid in color. The surface may be smootl and shiny or psendocutaneous.

External and internal piles often present themselves as nodules situated closely to each other and sometimes coalescing; thus larger tumors arise. In these hemorrhoidal varices important structural changes frequently take place. While at first soft, they may grow quite hard by the formation of blood clots or by a process of calcification. Inflammatory processes in the neighboring tissue have a tendency to increase their size and to make them more firm.

Internal piles are often pushed downward during the act of defecation. In this mauner the mucous membrane of the base of the tumor is subjected to greater traction, and thus ultimately a pedicle is formed. Such nodules provided with more or less long pedicles and situated near the internal sphincter very frequently slip ont from the anus at each defecation. When, however, they are not especially large, they spontaneously return into the rectum after defecation is finished. If they are of considerable size, it sometimes happens that they become incarcerated by the external sphincter, and if not carefully replaced, inflammation may develop and give rise to intense pains. Occasionally they may even become gangrenous and ultimately drop off. In some instances hemorrhoids undergo retrograde changes, become smaller, and even disappear entirely. Flaps of skin hanging near the anus and presenting a brownish color are often the remnants of previous piles. External piles sometimes give rise to the formation of warts and their surface assumes an uneven and wrinkled appearance. Interual hemorrhoids are often 
complicated by inflammatory processes of the neighboring tissues. Such processes give rise to the formation of ulcers, proctitis, and periproctitis. In the latter instance an abscess may be formed, which may open either externally or internally, sometimes both ways. Thus a complete rectal fistula originates.

In internal hemorrhoids the mucous membrane of therectum almost always exhibits the signs of a chronic catarrh. Its surface is swollen, succulent, and often covered with a thick layer of mucus. Occasionally there may be some pus. The proctitis accompanying piles may be either the cause or the sequel of the latter; sometimes, however, both may be due to some other factor.

Symptomatology.-Most of the symptoms produced by hemorrhoids are generally of a local character. They greatly vary in the different varieties of piles. In the early stage of external pile there occur off and on, especially after indiscretions in eating and drinking (principally effervescent wines or strong alcoholic beverages), attacks caused by an increased congestion of the hemorrhoids. These attacks may be described as follows: A sensation of fulness or clogging and slight pulsation in the anus are felt by the patient. Moderate constipation exists, compelling the patient to strain more than ordinarily. Itching of the anal region and the perineum frequently annoy the patient, especially soon after retiring, and may keep him awake for quite some time. On awaking in the morning the patient finds the anus tender and swollen, and after a movement a few stains of blood are discovered on the paper. Such an attack will, as a rule, pass off very quickly if the patient lives rationally and avoids the predisposing causes; if not, the attack will quickly recur with greater intensity and gradually assume a severer type. 
External piles may become swollen and cedematous, and are then extremely painful to touch. Sometimes there may be ulceration, or suppuration may take place and small painful fistulæ may form. The venous tumors now and then irritate the sphincter and levator ani muscles and produce spasm of the latter. The piles are then occasionally drawn up into the anus and pinched by the latter. This causes a great deal of pain and keeps the patient awake during the night. A feeling of throbbing and a sensation as of a foreign body in the anus exist. A frequent desire for defecation is thereby produced and the patient, as a rule, is inclined to attempt to expel the foreign body by forcible straining, which of course only aggravates the pain. Under these circumstances the patient can hardly sit down nor can he walk about comfortably, and on coughing and sueezing experiences great suffering on account of the constriction of the involved diseased parts. During a movement of the bowel, and for some hours afterward the pains are greatly increased. The patient is unable to attend to his daily occupation. General symptoms like fever, anorexia, dizziness, "severe constipation, may accompany the local manifestations.

Of the internal hemorrhoids, the capillary variety, being small and only slightly elevated above the mucous surface, gives rise to scarcely any trouble. As a rule, there is no pain. Occasionally, however, ulceration takes place which may cause considerable suffering.

Arterial and venous hemorrhoids give rise to many more symptoms. In case the sphincter muscles are relaxed, the hemorrhoids often protrude on the slightest exertion. This also often occurs at stool. At first they spontaneously return within the sphincter after the bowels have moved or whenever the exertion has ceased. Later in the 
course of the disease, however, the patient is compelled to return them with the finger. In still more advanced cases they never remain long within the sphincter and protrude very often whenever the least exertion is made. In this manner the hemorrhoids cause much discomiort. They also discharge a gummy acrid mucus which keeps the parts constantly moist and leads to excoriations around the anus, and also faror's the development of cutaneous excrescences. Patients with fully developed internal hemorrhoids experience a great deal of suffering during defecatiou. They also feel quite uncomfortable for some time afterward, occasionally to such a degree that they have to lie down. When walking they are always conscious of the fact that they have an anus.

In other instances in which the sphincter ani is strong and tight, the piles in coming down become nipped and their return is rendered difficult and painful.

The smptom from which the hemorrhoids originally derived their name, namely, hemorrhage, is common to all varieties of piles, although it is by no means constant. In many instances it is absent, or it does not play any essential part, especially in external piles. In some patients a more or less considerable hemorrhage takes place at certain intervals, appearing periodically, occasionally with great regularity. A few premonitory signs, consisting in painful sensations in the back and around the anus, constipation, and other indefinite nervous symptoms usually precede for a few days the beginning hemorrhage. The blood as a rule then appears at first in small quantities gradually increases in amount, and the hemorrhage stops on the fifth or sixth day after its commencement. Physicians in olden times-and some of the laity even nowadays - looked upon the hemorrhage as an important event, free- 
ing the system of vicious material. This is the reasou why formerly the hemorrhoidal bleeding was termed the golden flow. Nowadays we do not attach any particular importance to these hemorrhages. Their regularity or the periodicity of their appearance is simply attributable to the fact that the time necesary for the filling up of the nodules until they rupture is usually of the same leugth.

In some instances there are transient hemorrhages, lasting a shorter or louger period. Ordinarily the patients feel relieved after the bleeding; occasionally they remain quite well for a loug time, sometimes for a year or two, until there is suddenly a new hemorrhage. In the latter case the hemorrhage is commonly caused by some unusual occurrence; thus, a very copious meal, a loug ride ou horseback, or an excess in venery may bring it on.

In another class of patients there may be continuous small hemorrhages. These occur more frequently in cases of capillary hemorrhoids. The quantity of blood lost at each action of the bowel is small, but being steady it becomes a serious strain upon the patient's constitution and may give rise to severe forms of anæmia and even pernicious anæmia.

The blood discharged from piles is either of a bright red or a dark brown color, depending upon its origin from arteries or veins. It is characteristic of hemorrhoidal hemorrhages that the blood usually appears in a liquid, non-coagulated state, covering the fecal matter, but not mixed with it. If the hemorrhage is very copions, unconsciousness may result combined with symptoms of profound collapse. This, however, happens very rarely. Accompanying the local manifestations, especially if the latter are of a high degree, there may be varied general symptoms. Thus dyspnoea, palpitations of the heart, angina 
pectoris, irregular heart action, hiccough, headaches, giddiness, dizziness, buzzing in the ears, and cloudy vision may be present. Often a despondent feeling and a condition resembling hypochondria is met with. Anorexia, nausea, belching, and constipation also often occur. The general symptoms are especially marked if incarceration of internal piles within the sphincter has taken place. In case the swelling of the hemorrhoids is so extensive that a repositiou caunot be quickly effected, there may be present besides the local pains high fever and signs of collapse. If the incarceration lasts a long period, the hemorrhoids may become gangrenous and either fall off, accompanied by profuse hemorrhage, or, although rarely, give rise to septic and peritonitic conditions. In most instances after a falling off of the hemorrhoid a spontaneous cure takes place.

Some cases of hemorrhoids are complicated with catarrh of the rectum (proctitis). In such instances the stools reveal the presence of a considerable quantity of mucus, occasionally even of pus. Sometimes the mucous or muco-purulent fluid admixed with the fæces may be tinged with blood. These cases are often accompanied by a paretic condition of the sphincters, which allow the secretion to dribble from the anus. This gives rise to excoriations and inflammation of the anus and the neighboring tissues. In the course of the proctitis prolonged tenesmus may appear at times. If the inflammation extends into the rectal cellular tissue, it may lead to the formation of abscesses which may empty into or outside the bowels. This is the most frequent way in which fistulæ are produced.

Disturbances of the adjacent organs are also occasionally met with in cases of piles. Thus ischuria, stranguria, hemorrhages from the bladder, hemorrhages from the va- 
gina, and catarrhal conditions of the latter are encountered.

Diagnosis. - The diagnosis of hemorrhoids as a rule is easy. External piles are found by inspection of the anus, the patient lying on his side with the thighs drawn up. The buttocks are pushed aside with the hands, and the patient is instructed to strain in a similar manner as when having a stool. Nodules of a reddish-bluish tinge will be noticed in the immediate vicinity of the anns or partly within it. It is characteristic of hemorrhoidal nodules to increase in size during a period of constipation, and to diminish after an efficient evacuation of the bowels.

Condylomata and small skin tags around the anus can be easily differentiated from piles. Condylomata, as a rule, encircle the anus and are present also on other parts of the body, especially on the scrotum. Besides, there will be a previous history of syphilis, and occasionally other luetic manifestations. The cutaneous tags present more the appearance of whitish-looking skin, never change in size, and do not bleed when punctured, while hemorrhoids bleed profusely on puncture.

The diagnosis of internal hemorrhoids can be made by a digital examination or by this in connection with the inspection of the lower portion of the rectum by means of a speculum. The characteristics of internal piles are similar to those of external hemorrhoids. They can be easily differentiated from polypi by means of puncture with the needle. Polypi do not bleed when punctured. Besides, polypi are usually found in children, while hemorrhoids occur with greatest frequency in the advanced period of life.

Carcinoma of the rectum will rarely give rise to mistakes, the tumor usually presenting a much harder consistency 
than hemorrhoids. As a rule, there will also be other signs of a malignant trouble, cachexia, etc. It is needless to say that cancer of the rectum may be combined with hemorrhoids. As a matter of fact, it very often gives rise to their development, and the discovery of piles which have formed within a short period of time should indeed rouse the suspicion of cancer of the rectum.

Progmosis.--The prognosis of external as well as interual piles is as a rule favorable. They generally exist for a long time, not infrequently throughout life. They hardly ever endanger life, unless some grave complications (incarceration of the hemorrhoids or gangrenous processes or very profuse hemorrhages) superrene. Hemorrhoids are liable to recede or even to disappear entirely, especially if the factors producing them have been eliminated.

Treatment.-A rational mode of living is of the greatest importance. Patients with hemorrhoids should have plenty of outiloor exercise, should partake of food with moderation, should avoid all excesses in baccho and in venere, and should endeavor to have a daily evacuation of the bowels. Any condition eausing venous hyperæmia of the rectum must be removed. Thus rocations requiring constant sitting, or constant standing, or horseback riding should be entirely or partly given up.

With regard to diet the following general rules may be given: Patients with hemorrhoids should avoid copious meals. They should rather eat of ten and sparingly. Fish, fresh, well-cooked regetables, and ripe fruit should form a considerable part of their diet. Alcoholic beverages, strong coffee, and highly seasoned dishes should be avoided. The different kinds of cheese, very coarse brown bread, cabbage, peas, and beans are best eliminated from the diet. Salads, potatoes, beets, spinach, asparagus, cauliflower, are, how- 
ever, rather of benefit if taken in small quantities, as these artjcles make the intestinal contents more liquicl. Sterred fruits and also raw fruit, as for instance apples, pears, prunes, oranges, grapes, are useful. As a beverage, plain water, best taken between meals in the quantity of a pint, is most beneficial. In some instances, especially in anæmic patients, buttermilk in the same quantity may be taken instead of water. A small amount of light beer is permissible in some cases.

With reference to hygiene or prophylactic measures it is of importance for the patients to have plenty of outdoor exercise, especially walking. The exercise, however, should not be continued to over-fatigue. Gymnastic exercises at home, sawing or chopping wood, and the like, and also massage are best adapted for this purpose. The patients should wash the affected part in the morning and evening witl cool water. They should sit on caned chairs, not on upholstered ones, and should sleep on a mattress.

The patient should have a good evacuation of the bowels daily. In case this does not occur, it will be of the greatest importance to secure it by the different therapeutic measures at our disposal (see Chapter $\mathrm{X}$., on constipation). As a rule, however, powerful laxative and drastic remedies should be avoided. The frequent use of injections had also best be omitted. The purgatives most adapted for these patients are the saline ones, sulphur and rhubarb drugs. Thus componnd licorice powder, a teaspoonful in the evening, or sulph. depur., potas. bitartrat. ai, also one teaspoonful in the evening. Rhubarb in the form of tincture or in substance 0.5 to $1 \mathrm{gm}$., taken once or twice daily, is also advantageous for a prolonged use. The waters of Carlsbad, Kissingen, Marienbad, Tarasp, Saratoga, will also be of benefit, especially if taken at the 
watering-places themselves in connection with a prescribed diet. If the hemorrhoids have already attained considerable size, local remedies will often be required.

Local Treatment. - The irritation or the rubbing of the piles against each other or against the skin must be prevented. For this purpose covering the piles with a small piece of smooth and clean cotton is of benefit; still better, however, for this purpose is cotton moistened in olive oil or covered with vaseline or a soft salve (Hebra's ointment or ointments of zinc, lead, boracic acid). If the piles are inflamed, it is best to first paint them a few times with the following solution:

R Potas. iodidi ........................ 2.0 (3 ss.)

Iodi puri.......................... 0.2 (gr. iiiss.)

Glycerin........................ 40.0 (3 x.)

before applying the ointment. After a movement the anus and the piles should be first washed with cool water and then wiped off with soft cotton or linen. This must be done very gently. Persous suffering with annoying tenesmus after defecation should accustom themselves to go to stool before retiring. The recumbent position which the patients are thus able to assume soon after the passage affords them decided relief.

If there are pains in the rectum caused by a mere hyperxesthesia of the mucous membrane, an injection of one to two teaspoonfuls of warm olive oil or of the same quantity of warm water into the bowel will exert a favorable influence. If this fails, or in cases in which the pains are caused by a superficial excoriation of the piles, it is best to apply an ointment containing some narcotic after an evacuation of the bowels, and sometimes even during the intervals. The following salve, recommended by Rosenheim, is very appropriate: 
R Lanolin............................ 20.0 (3 $\mathrm{v}$.)

Bism. subnitr....................... 2.0 ( 3 ss.)

Extr. opii........................... 0.3 (gr. v.)

M. f. ungt.

In place of the ointment the piles may be painted with a solution containing equal parts of fluid extracts of opium and belladonna, or with a two-per-cent cocaine solution. Suppositories containing opium, belladonna, or cocaine are also effectual.

Internal piles prolapsing through the anus should be pushed back by the patient after anointing them with olive oil or with vaseline. In case the reposition is not easy, painting of the piles with a two-per-cent cocaine solution will after a while lessen the sensitiveness and thus make reposition possible. In some obstinate cases the patient must be narcotized in order to accomplish this. If the incarcerated piles have already become gangrenous, the pains usually grow less. In order to arrest the necrotic process it is advisable to dust the affected area with an antiseptic powder (dermatol) and to cover it with dry gauze. The pile usually falls off spontaneously and the wound heals of itself.

The inflammatory processes in piles require special treatment in the stage of exacerbation (general antiphlogistic remedies). Thus rest in bed on the side, application of cold in the form of an icebay or a Priessnitz poultice, occasionally leeches in the neighborhood of the anus, not on the piles themselves. Application of cold lead water is also useful. In case there are signs pointing to the formation of pus or the development of a septic process, surgical intervention is imperative. An incision into the hardened piles followed by thorough extirpation is essential. Inasmuch as such an operation must be dene under chloroform narcosis, the radical removal of the eutire hemorrhoidal area is therefore best performed at the same time. 
Hemorrhoidal hemorrhages, if not extensive, and if occurring at long intervals, will hardly require any therapeutic measures. If, however, the quantity of blood is quite considerable or if the hemorrhage is protracted, the following means should be employed: An icebag should be applied to the anus for several hours, or in case the hemorrhage results from internal piles, a cylindrical piece of ice is pushed up into the anus and replaced every half-hour. The rectal refrigerator may likewise be used with benefit. Very cold injections are also useful. In cases with very frecquent hemorrhages injections of water, to which an astringent remedy has been added, are beneficial. Thus a two-per-cent solution of tannic acid or of alum, or a 0.3per-cent solution of acetate of lead may be applied. The following ointment, first suggested by Kossobudskj, ${ }^{2}$ may also be applied in these cases:

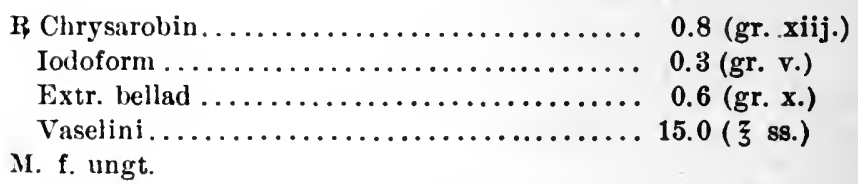

This salve not only checks the hemorrhage, but has also an excellent effect in reducing the size of the pile. In internal hemorrhoids the following suppository may be used for the same purpose:

R Chrysarobin......................... 0.1 (gr. ig

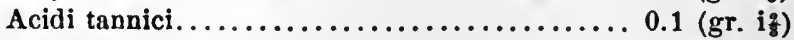

Iodoform.............................. 0.2 (gr. iiit)

Extr. opii.......................... 0.02 (gr. $\left.\frac{1}{8}\right)$

Ol. theobrom....................... 2.0 (3 ss.)

M. f. Suppository. S. One suppository in the evening.

' Kossobudskj : Centralblatt für Chirurgie, 1889. 
Radical Treatment.-1. Dilatation of the Sphincters. Verneuil ' was the first to recommend dilatation of the sphincters as a cure for piles. This treatment is based upon the idea that the spasm of the sphincter is thereby stopped, that the bowels act more freely and the pressure upon the venous blood-vessels is relieved. The dilatation of the sphincters may be accomplished gradually by introducing. specula into the rectum, taking a larger size each time, which procedure occupies several weeks, or it may be done in one sitting (the so-called forcible dilatation). In the latter instance, however, chloroform narcosis is necessary. Complete dilatation is effected, according to Allingham, in the following way: The patient being fully under the influence of ether or chloroform, both thumbs must be inserted into the rectum, which is to be dilated gradually, first in the antero-posterior and afterward in the opposite direction. The amount of force used must be sufficient to overcome the spasm thoroughly. This manipulation must be continued until the sphincter muscles yield, as if reduced to a really pulpy condition. Care must be taken to act high enough up in the rectum so as to include the whole of the sphincter. The result is that the state of contraction is abolished and no spasm can occur. In fact, for the time being, as in any other stretched muscle, paralysis results. With great gentleness the desired effect may be accomplished without tearing the mucons membrane. But some extravasation is usually noted around the anus for a few days. After this an opium suppository is kept in the rectum and the patient is placed in bed in a recumbent position. Dilatation of the sphincters may be recommended in the early stage of hemorrhoids, especially in cases combined with constipation; further in hemorrhoids

${ }^{1}$ Verneuil : Gazette des hôp., 1884, 1887. 
during pregnancy or occurring in persons greatly debilitated by other grave diseases.

2. Carbolic-Acid Injections. Pooley, ${ }^{1}$ Kelsey,${ }^{3}$ Roux,${ }^{3}$ and Lange " have recommended injections of carbolic acid into the piles in order to produce shrinking. This method is permissible only if the hemorrhoids are not inflamed. Proceed as follows: The piles are first thoroughly cleansed and dried, then corered with iodoform salve. In order to lessen the pains a few drops of a one-per-cent cocaine solution may first be used subcutaneously. Then three to five drops of either of the two following solutions are injected into the centre of each pile: (1) Carbolic acid 1, glycerin 3; (2) Carbolic acid 1, glycerin 3, distilled water 3. The injection is made with the common Pravaz syringe, but care must be taken that none of the solution drips from the needle, so as to aroid cauterizing the mucous membrane. Sereral piles can be treated at the same sitting. It is advisable, however, not to make the injections oftener than about once a week. This procedure if carefully done is not dangerous nor painful, and often effects shrinking or even disappearance of quite considerable hemorrhoidal nodules.

3. Cauterization with Fuming Nitric Acid. Houston, ${ }^{5}$ of Dublin, was the first to recommend cauterization of piles with fuming nitric acid. This may be done in the following manner: After thorough cleansing and drying of the anus and the surrounding parts, the entire area is covered

'J. H. Pooley : "Injection of Carbolic Acid in Hemorrhoids." Toledo Med. and Surg. Journal, November, 1877. No. 11.

${ }^{2}$ Charles B. Kelsey : "The Treatment of Hemorrhoids." Medical Record, 1886, vol. ii., p. 141.

'Roux: "Behandlung der Hämorrhoiden." Therap. Monatshefte, 1895 , p. 124.

F. Lange : Centralblatt für Chirurgie, 1887, No. 25. Beilage, p. 70.

${ }^{5}$ Houston: Dublin Journal of Jedicine, 1844. 
with a thick layer of vaseline excepting the pile which is to be treated. The latter is then painted with nitric acid by means of a small stick of wood or a glass rod. Special care must be taken that the acid reaches no other spot. After the nodule has assumed a grayish-green color it is carefully dried, smeared with vaseline, and pushed back into the rectum. This method is best adapted for smaller nodules, especially if they have a wide base. Sometimes a second cauterization is necessary, which may be done after an interval of about five days or a week. Instead of nitric acid other cauterizing substances may be used, and Allingham has recommended concentrated carbolic acid as especially efficient for this purpose.

4. Ligature. Cooper ${ }^{1}$ recommended the ligature of hemorrhoids in order to cut them off from the circulation and thus destroy them. Salmon ${ }^{2}$ has improved this method by making an incision before applying the ligature. According to this writer, the operation is performed in the following manner: The patient is placed on the right side on a. hard couch and is completely anresthetized. The sphincter muscles are then gently but completely dilated. The hemorrhoids, one by one, are then drawn down with a pronged hook fork; by means of sharp scissors the pile is separated from its connections with the muscular and submucous tissues upon which it rests. The cut is best made in the sulcus or white mark which is seen where the skin meets the mucous membraue. This incision is made in a direction parallel to the bowel and carried to such a distance that the pile is left connected by an isthmus of ressels and mucous membrane only. A well-waxed, strong, thin, aseptic silk ligature is now placed at the bottom of the deep

1 Cited from Allingham, loc, cit.

${ }^{2}$ Ibid. 
groove which has been made, and the ligature is tied right at the neck of the tumor as tightly as possible. When all the hemorrhoids have thus been ligated, they should be returned within the sphincter. A small piece of absorbent cotton saturated with iodoform ointment is now placed into the bowel and a pad of cotton applied over the anus.

5. Cinshing. Crushing of piles has been suggested by Pollock and the method further improved by Allingham, ${ }^{1}$ who devised a very ingenious apparatus for this purpose, namely, the "screw-crushing instrument." The operation begins witl the dilatation of the sphincters. The hemorrhoid is then drawn into the screw-crusher by means of a hook, and this being intrusted to an assistant the bar is pushed up and screwed home as tightly as possible. The pile should be crushed longitudinally and not transrersely. The projecting portion of the pile is cut off with the knife or scissors and the pressure kept up for about one minute. According to Allingham crushing is a very satisfactory method of removing internal piles.

6. Thermo-cantery (Paquelin) and Galvano-cautery. Langenbeck introduced the method of operating upon piles by means of thermo-cautery. Each pile is seized with a volsellum forceps and drawn well down. The clamp is then applied so as to embrace its base. The portion above the clamp is cut off with a pair of scissors and the cauteryiron, heated to a dull red heat, is repeatedly applied to the stump until all the vessels are well seared.

Instead of using the Paquelin, galvano-cautery may be applied for the removal of hemorrhoids, the technique being identical with the former. Bardeleben and also Rosenheim strongly recommend the latter method.

'Allingham: "Diseases of the Rectum." 1896, p. 153. 
7. Extirpation of Hemorrloids, followed by Suture. This method was first introduced by von Esmarch ${ }^{1}$ in Germany and by Whitehead in England. It is not, however, extensively used as it is quite complicated, besides giving rise to many disagreeable complications. Thus Allingham has noticed the following sequels of such an operation: 1. Anal stricture. 2. Loss of sensation and control over the anus. 3. Irritation of the mucous membrane due to frequent discharges of mucus and at times accompanied by bleeding.

After any of the above-uamed operations it was customary to employ an astringent in order to prevent a movement of the bowels for a few days. Contrary to this method E. Graser ${ }^{2}$ is of the opinion that such patients are better off when having a free movement shortly after the operation. $\mathrm{He}$ administers soon after its performance a small dose of castor oil and instructs the patient to have an evacuation while in a warm sitz bath. Cleansing of the anus is rery easily obtained in this mamer. After an antiseptic washing a piece of cotton or linen, thickly smeared with an ointment, is introduced into the rectum. This procedure has usually to be performed once daily. According to Graser, the patients if thus treated are almost without pain, and are able to get up and be out of bed five or seven days after the operation. For some time after its performance it is advisable to have the patient introduce bougies of varying size into the rectum in order to prevent the formation of a stricture.

Complications. - Prolapse of the Rectum. Prolapse of the rectum is a frequent complication of hemorrhoids, although 'von Esmarch: "Die Krankheiten des Mastdarms und Afters," Stuttgart, 1887.

${ }^{2}$ E. Graser : Penzoldt u. Stinzing, "Handbuch d. Therapie, "Bd. iø., p. 634 . 
it may also occur alone. The prolapse may involve either the mucous membrane alone or all the coats of the rectum. In the latter instance this condition is also called procidentia recti. Outside of the anus there is a protrusion of the mucous membrane in its entire circumference. An internal prolapse of the rectum may also occur, which consists in the descent of the upper part of the rectum through the lower part, but not appearing outside the anus. This corresponds rather to an intussusception. A relaxation of the ligaments which serve to keep the rectum in its place is often the cause of this malady. Weakness and paralysis of the sphincter ani muscles are also predisposing factors.

Prolapse of the rectum is frequently found in debilitated children, especially if an intestiual catarrh is present, for these little patients go to stool too often and usually strain too much and for too long a time. These conditions weaken the muscular apparatns of the anus, and thus a prolapse of the rectum easily arises. In elderly people, in patients suffering from affections of the bladder or from severe constipation and internal hemorrhoids, and in women who have gone through many pregnancies in quick succession, prolapse of the rectum is also a frequent occurrence.

The symptoms are as follows: If the prolapse is only of a moderate degree, there appears in the act of defecation a protrusion of the rectum outside the anus, one or one and a half inches in length, the mucosa looking quite red and puckered. In the more advanced stage the bulged out rectum resembles a large tumor with a star-like opening at its centre, while the color is pale or bluish-red. In children the mass generally protrudes only on going to stool, but in adults it is constantly down' or comes down on the slightest exertion, and therefore may become ulcer- 
ated or inflamed. In old cases of prolapse incontinence of fæces is also frequently present.

The diagnosis of prolapse of the rectum is easily made from the above-mentioned appearances. Internal prolapse is nct so easily diaguosed, as the mass never appears outside the anus. This condition can be recognized only by means of a digital examination of the rectum. The finger introduced into the bowel is first kept close to the anterior or posterior wall, and is passed up until it meets with an obstruction (i.e., it has passed into the cul-de-sac). Then the finger is slightly withdrawn and the centre of the gut examined until an orifice is found into which the finger or a bougie may be passed for some inches high up into the rectum. If the intussusception is rather far up in the rectum, the patient should bear down during the examination.

With regard to treatment it is of importance to eliminate all the conditions which were predisposing factors for the prolapse. Extreme cleanliness, especially after defecation, should be observed. The reposition of the prolapse should. be performed in the most careful manner. It is best done in the knee-elbow posture. If a considerable portion of the bowel has come down, a large flexible bougie may be passed into the bowel in such a manner as to carry before it the upper part of the descended gut. General taxis should at the same time be used, and in this way the mass can generally be returned. In cases in which the prolapse occurs quite frequently, even during a walk, a rectal supporter, as suggested by von Esmarch, should be worn by the patient. It consists of a soft-rubber ball attached to the anus by means of a belt and a $\mathrm{T}$ bandage.

The palliative treatment which is especially successful in children is as follows: All sources of irritation shonld be removed and tho general health strengthcued. Straining at 
stool should be strongly forbidden and a mild laxative remedy given. After a movement of the bowels the protruded part should be well washed with cold water and pushed back into the anus by gentle pressure. After this procedure the patient should remain in a recumbent position for half an hour or so, best lying on the abdomen. If these means alone are not sufficient, the following more radical measures will have to be adopted: Cauterization of the prolapsed part with fuming nitric acid or with the thermo-cautery under chloroform narcosis is often of great benefit. Care should be taken while cauterizing not to touch the verge of the anus or the skin. After this the. prolapsed part should be well oiled and returned. Instead of nitric acid Allingham uses the acid nitrate of mercury.

These cauterizing methods have the disadvantage of often producing strictures of the rectum. For this reason a number of surgical operations have been devised. Thus excision of triangular or elliptical portions of the mucous membrave, bringing the edges together with sutures, has been practised. Extirpation of the entire prolapsed portion was first advocated by Treves. ${ }^{1}$ F. Lange, ${ }^{2}$ of New York, has described a new operation, serving the purpose of reducing the calibre of the rectum and at the same time producing a narrow muscular ring. The patient is placed in the genu-pectoral position, an incision is made from the lower part of the sacrum down to the anus, until the posterior wall of the rectum is reached; the coccyx is then removed. The object in view is to narrow the gut as high as possible and to lessen the impediments to the action of the levator ani. The calibre of the rectum is lessened by introducing buried étage sutures of iodoformed catgut, which do not

'Treves : Lancet, 1890, vol. 1.

'F. Lange: Annals of Surgery, vol. v., p. 497. 
perforate the entire thickness of the gut. The first rows are inserted near the middle line and form a fold in the posterior walls which protrudes into the bowel. In this manner the more lateral portions of the gut are brought into position without causing too much tension. Similar sutures are applied to unite the cut surfaces of the levator ani and sphincter exteruus, which had been previously dissected in order to lay bare the posterior wall of the rectum. The cavity thus formed is filled up with iodoform gauze and the flaps of integument are united with sutures.

Another very efficient operation has been suggested by Allingham and cousists in making a small incision through the anterior abdominal wall on the left side, just abore the outer third of Poupart's ligament, then introducing the fingers into the abdomen, catching hold of the rectum and pulling it up. After it has been drawn as high up as possible, silk threads are passed through the mesentery aur the latter is fastened to the abdominal wall.

Fissure of the Anus. Another affection which very frequently occurs in counection with hemorrhoids is anal fissure. The latter consists of an oblong tear of the mucosa of the anus and gives rise to severe pain and spasmodic contractions of the sphincters. Fissures or ulcers of the anus vary in depth and size. Some are mere abrasions of the mucous membrane, others are quite large and deep so that the muscular fibres are laid bare. The edges of the fissure may be in a healthy state or they may be inflamed, callous, and indurated. Fissure of the anus is usually caused by an injury or tearing of the mucous membrane at the verge of the anus. This may result either from excessive straining or from the passage of very dry hard scybala. The affection is more often found in women than in men. The posterior portion of the anus is the point of 13 
predilection, although the fissure may occur at any other place. It is usually situated parallel to the external sphincter, although in some instances it may lie higher up, parallel to the internal sphincter or even above it.

The symptoms consist in intense pains in the rectum on defecation, sometimes persisting afterward. The pains are often of a very excruciating character. The size of the fissure does not seem to be of so much importance with regard to the severity of the pain as its position. A small crack situated at the anal orifice over the external sphincter and involving the skin canses much greater pain than a large nlcer situated higher up in the rectum. There may also be a discharge of blood and pus.

The diagnosis of anal fissure is made by the symptoms just mentioned and by local examinations. The patient lying on his left side should be told to bear down, and the anus opened with forefinger and thumb as gently as possible. An elongated club-shaped nlcer will be seen within the orifice. Its floor may be very red and inflamed, or if the ulcer is of long standing, of a grayish color, with welldefined and hard edges. Often the introduction of the finger into the anus is so painful that before making the examination a suppository containing one grain of cocaine has to be applied. Sometimes even this procedure is insufficient, and then chloroform anesthesia will be required. For a fissure situated higher up above the internal sphincter examination with the speculum will have to be made.

Fissures of recent origin can often be cured without any operation. Rest in the recumbent position should be adopted as much as possible. Mild laxatives are to be recommended, but no drastic remedies employed. If the patient can manage to have a movement at night time before retiring, it will be of advantage. Locally. the fissure 
should be touched off and on with a ten-per-cent solution of cocaine or with a ten-per-cent solution of nitrate of silver. Still better is the application of the following salve recommended by Allingham:

R Hydrarg. subchlor.......................... gr. iv.

Pulv. opii.............................. gr. ij.

Extr. bellad............................. gr. ij.

Ung. sambuci............................. $3 i$.

M. f. ung.

If these palliative remedies are not sufficient, a free incision through the fissure should be made. The cut should be rather deep and should reach the sphincter muscles. 


\section{CHAPTER VIII.}

\section{APPENDICITIS.}

Synonyms: Scolecoiditis; Perityphlitis; Paratyphlitis; Appendicular inflammation.

Definition. - Inflammation of the appendix, characterized by localized pains, commonly fever and digestive disturbances.

General Remarks. - The inflammatory lesions involving the right iliac region were formerly designated as typhlitis (inflammation of the cæcum itself), perityphlitis (inflammation of the peritoneal corering of the cæcum), and paratyphlitis (inflammation of the retro-peritoneal connective tissue of the cecum). Grisolle ${ }^{1}$ was the first to maintain that inflammation of the creum could hardly give rise to such grave lesions as are found in the right iliac fossa, for even ulcerations of the cæcum and colon do not, as a rule, show any tendency to extend into the neighboring connective tissue. He ascribed the above conditions to an inflammation of the appendix, which organ shows a tendency to perforate and to lead to abscesses in the right iliac fossa as verified by post-mortem examinations. The possibility of a stercoral typhlitis (inflammation of the crecum as the result of accumulated fecal matter) which was formerly generally accepted, is now held by but very few writers,

${ }^{1}$ Grisolle: "Tumeurs Phlegmoneuses des Fosses lliaques." Archives de Médecine, 1839. 
as for instance, Lennander. ${ }^{2}$ Sahli, ${ }^{2}$ Nothnagel, ${ }^{3}$ Fowler, ${ }^{4}$ Sonnenburg, ${ }^{6}$ and others deny its existence. The teachings of Grisolle found further support through the brilliant investigations of Reginald Fitz ${ }^{6}$ of Boston, Sands, ${ }^{7}$ McBurney, ${ }^{6}$ Weir, ${ }^{\circ}$ Bull, ${ }^{\circ}$ and Fowler of New York, were supplemented by the observations of Sonnenburg, Sahli, Rotter, "Roux, ${ }^{12}$ Talamon, ${ }^{13}$ and others, and are now generally accepted.

Etiology.-In former years much importance was attributed to the occurrence of foreign bodies like cherry stones, grape seeds, lemon and orange pits, date kernels, fish bones, pins, etc., within the appendix as causative factors of the inflammatory suppurative process. According to Fowler, the belief that the disease is frequently due to the engaging of foreign bodies in the cavity of the organ is based to a large extent upon purely speculative or imaginary conditions or erroneous observations. In a very large number of cases of this disease upon which he operated Fowler found but in two instances any body other than soft fecal masses which could be considered as being in

1 Lennander: "Ueber Appendicitis," Wien, 1895.

${ }^{2}$ Sahli : "Ueber das Wesen und die Behandlung der Perityphlitiden." Correspondenzbl. f. Schweizer Aerzte, Basel, 1892.

${ }^{3}$ Nothnagel: "Krankheiten des Darms," Wien, 1898.

${ }^{4}$ George R. Fowler: "A Treatise on Appendicitis," Philadelphia, 1894.

5 Sonnenburg: "Pathologie und Therapie der Perityphlitis," Leipzig, 1895.

'Reginald Fitz: American Journal of the Medical Sciences, 1886; and New York Medical Journal, 1888.

'Sands : New York Medical Journal, 1888, p. 197-205, 607.

${ }^{8}$ Charles McBurney : Annals of Surgery, 1891; Medical Record, 1892.

${ }^{9}$ Robert F. Weir : Medical Record and Medical News, 1887-1892.

${ }^{10}$ W. T. Bull : Medical Record, 1894.

"Rotter: "Ueber Perityphlitis," Berlin, 1897.

12 Roux : Revue de Médecine de la Suisse romande, 1890, 1891, 1892.

${ }^{13}$ Talamon: "Appendicite et Perityphlite," Paris. 1892. 
any seuse foreign. The fecal concretions within the appendix are now looked upon as of no importance whatever with regard to the causation of the disease, as they are also accidentally encountered in perfectly normal appendices. The opinion generally prevails that the inflammation is caused by micro-organisms which are conveyed to the interior of the organ in the fecal matter. According to Nothnagel, however, fecal concretions play a prominent part in lesions leading to perforation of the appendix.

Morable kidney has been assumed to be a predisposing factor in the development of appendicitis by Carl Beck ${ }^{\text {* }}$ and Edebohls. ${ }^{2}$ The much greater frequency of movable kidney in the female and the comparative infrequency of appendicitis in the latter as compared with the male sex seems to speak somewhat against this view.

Actinomycosis, tuberculous and typhoid ulcers are predisposing causes of the disease. Occlusion of the lumen of the appendix, either partial or complete, is likewise a predisposing factor. These occlusions may be the result of former inflammatory lesions, but are most frequently due to the retrograde changes which this organ is gradually undergoing in the process of evolntion. According to Ribbert ${ }^{3}$ and Zuckerkandl, ${ }^{4}$ the appendix is found obliterated in about twenty-fire per cent of all living persons. Both these writers ascribe this condition not to inflammatory diseases, but to the progress of evolution which takes place in the appendix. This riew is supported by the fact that

'Carl Beck: "Appendicitis." Volkmann's Sammlung klinischer Vorträge, No. 221, Leipzig, 1898.

${ }^{2}$ George M. Edebohls : Medical Record, 1898.

${ }^{8}$ Ribbert: "Beitriige zur normalen und pathologischen Anatomie des Wurmfortsatzes." Virch. Arch., Bd. 132.

4E. Zuckerkandl: "Ueber die Obliteration des Wurmfortsatzes beim Menschen," Wiesbaden, 1894. 
obliteration of the appendix is found with gradually increasing frequency in more advanced age. Thus Ribbert found obliteration of the appendix in fifty per cent of persons above sixty years of age.

Why the appendix should be the seat of disease so very much more frequently than other parts of the intestine is a question which cannot be so easily answered. The fact that the appendix is a rudimentary organ in which processes of evolution are even normally discoverable makes it probable that it is imbued with less resistance against disease-producing agents. The comparatively narrow lumen of the appendix and Gerlach's valve make the emptying of this little canal a difficult matter. This, in connection with the scantiness of circular muscular fibres in the walls of the appendix explains the slowness with which substances within the appendicular cavity are emptied into the intestine. Stagnation of contents in this organ is certainly a predisposing factor for disease. The abundance of adenoid tissue in the appendix has been believed ly some writers to be a predisposing cause of disease. Bacterial infections here take place in a similar manner as in the tonsils, and Sahli speaks by way of comparison of an angina of the appendix. Fowler and Van Cott ' believe that the vascular arrangement of the appendix (scantiness of blood supply, the main ressels being almost end arteries) is responsible to a great extent for the frequency of disease in this organ. Some of the blood-ressels and nerves are primarily affected, and the nutrition of the appendix being thus disturbed, diseases of an infective character easily take place. Another predisposing cause of appendicitis is displacement and malformation of the appendix.

While all the above-mentioned factors may predispose

1 Van Cott.Fowler: "Treatise on Appendicitis." 
the appendix to disease, the real cause of the latter must be looked for in a bacterial invasion. Talamon was the first to lay stress upon the importance of microbes in appendicitis. Nowadays all writers coincide with this view. Thus Tavel, ${ }^{1}$ Hodenpyl, ${ }^{2}$ Fowler, Wilson, ${ }^{3}$ Barbacci, ${ }^{4}$ and others ascribe a very important part to the bacillus coli communis (Escherich), which is almost always encountered in lesions of the appendix, either in the exudate, pus, or the walls of the appendix itself. Other micro-organisms are, however, frequently found either in connection with the bacterium coli commune or alone. Thus streptococcus pyogenes, pueumococcus, staphylococcus pyogenes aureus, bacterium lactis, bacillus pyocyaneus and pyogenes futidus, protens vulgaris, and others have been encountered. In most cases probably a mixed infection (several varieties of micro-organisms) takes place. The bacterium coli commune, however, is most frequently found, as it has a greater resisting-power and in the course of its growth usually canses disappearance of the other micro-organisms.

Sex and age seem to play an important part in regard to the distribution of the disease. The male sex is much more frequently affected than the female. Thus,

\begin{tabular}{llrlllll} 
Sonnenburg reports & \multicolumn{4}{c}{130 cases -77 males, } & 53 females. \\
Rotter & " & 68 & " & -44 & " & 24 & " \\
Nothnagel & " & 130 & " & -105 & " & 25 & " \\
Bamberger & " & 73 & " & -54 & " & 19 & "
\end{tabular}

\footnotetext{
'Tavel und Lanz: "Ueher die Aetiologie der Peritonitis." Mittheilungen aus Kliniken und Instituten der Schweiz, Basel, 1893.

2Hodenpyl: "On the Etiology of Appendicitis." New York Medical Journal, 1893.

3 E. Wilson : Cited after Fowler.

4 Barbacci : Lo sperimentale, 1893, fasc. 4.

'Bamberger: "Die Entzilndungen der rechten Fossa iliáca." Wiener med. Wochenschr., 1853.
} 
Volz ${ }^{1}$ reports 59 cases -45 males, 14 females. Matterstock * " 1,030 " $\quad-733$ " 297 "

This preponderance of the male sex is already found in early life. Thus Matterstock observed 72 cases of appendicitis in early life (seven months to fifteen years), and among this number were 51 male children and 21 girls. The greater frequency of appendicitis in the male sex is explained by Van Cott as due to the circumstance that the appendix of the male has a less abundant blood supply than that of the female; for in the latter there is a collateral circulation derived from the sexual apparatus.

With regard to age all writers agree that appendicitis is most frequently encountered between the tenth and thirtieth years. It occurs less frequently in the first decade of life and in the thirtieth to fortieth years, and is quite rare in advanced age. The following table is submitted with a view of showing the frequency of appendicitis in the different decades of life as recorded by several eminent writers :

\begin{tabular}{|c|c|c|c|}
\hline Ages. & Fitz. & Matterstock. & Nothnagel. \\
\hline Total number ............ & 228 & 474 & 129 \\
\hline 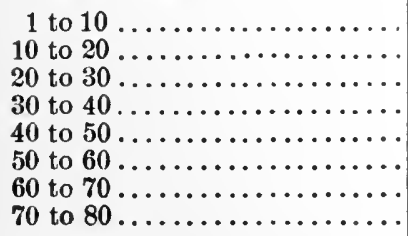 & $\begin{array}{r}22 \\
86 \\
65 \\
34 \\
8 \\
11 \\
1 \\
1\end{array}$ & $\begin{array}{r}46 \\
143 \\
158 \\
72 \\
30 \\
18 \\
5 \\
2\end{array}$ & $\begin{array}{r}1 \\
44 \\
57 \\
14 \\
7 \\
4 \\
2 \\
0\end{array}$ \\
\hline
\end{tabular}

The frequency of appendicitis in relation to other diseases can be studied from the report of the autopsies made

${ }^{1}$ Ad. Volz: "Die dureh Kotlssteine bedingte Perforation des Wurmfortsatzes, etc., " Karlsruhe. 1846.

${ }^{2}$ Matterstock: "Perityphlitis." Gerhardt's Handbuch der Kinderkrank., Thbingen, 1880. 
in the pathological institute of the Wiener Allgemeine Krankenhaus between 1870 and 1896. According to Nothnagel, the total uumber of autopsies was 44,940. Among these the number of cases dying from appendicitis amounted to 148 . The percentage of appendicitis, therefore, was 0.32 . With regard to sex there were 107 males (72.3 per cent) and 41 females (27.7 per cent). The actual frequency of appendicitis among the living, however, is much greater than appears from these numbers, which relate only to cases which have resulted fatally.

Morbid Anatomy. - The pathological anatomy of appendicitis has been thoroughly studied recently, not only in autopsies but principally in operative cases. In the latter an insight is permitted into the changes which take place early in the disease. Fowler distinguishes four stages of anatomical lesions according to the spread of the morbid process involving the different tissues of the appendix. In the first stage (endo-appendicitis) more or less intense inflammation of the mucous and submucous layers takes place. The second stage (parietal appendicitis) consists in an inflammatory process involving the interstitial or intermuscular structure of the body of the appendix. The third stage (peri-appendicitis) means an inflammatory process involving all the layers of the appendix, the peritoneum included. The fourth stage (para-appendicitis) consists in lesions involving the appendix and the neighboring tissues. This process is most often accompanied with suppurative inflammations of the connective tissue adjacent to that portion of the appendix which is not covered with peritoneum.

According to Fowler, the above described stages are not essentially different processes but further developments of one and the same lesion. 
Riedel, ${ }^{2}$ Nothnagel, and others distinguish two different types of appendicitis which are of great clinical importance. They are the following:

1. Catarrhal appendicitis (endo-appendicitis). Here in the acute form the mucosa of the appendix is swollen and reddened, the submucosa is engorged and filled with round cells. The follicles are distinctly swollen. The appendix appears swollen and more rigid, and its lumen is filled with thick yellowish contents, mostly mucus; sometimes the latter may be mixed with fecal matter. Occasionally there are fecal concretions. Often ecchymoses of the mucosa occur, leading sometimes to superficial defects (erosions). All these lesions may entirely disappear after the acute attack is over, and thus a perfect cure may be established. This, however, is possible only if there is no occlusion of the lumen of the appendix and the inflammatory products can be emptied into the crecum.

In the large majority of cases of catarrhal appendicitis the cure is not a perfect one and chronic appendicitis is the result. In this stage the mucosa of the appendix presents a slate-gray appearance. It is filled with accumulations of round cells; at the same time proliferation of connective tissue and occasionally blood pigment are found. The submucosa and muscularis may show no changes whatever, although as a rule they are hypertrophied. The latter condition is probably due to stricture of the lumen of the appendix and consecutive muscular (compensatory) hypertrophy. The chronic form of appendicitis, owing to suppurative processes of the mucous membrane, occasionally leads to a total destruction of the mucosa, and an obliteration of the lumen of the appendix. This condition is

1 Riedel: "Ueber die Frühoperation bei Appendicitis purulenta seu gangraenosa." Berl. klin. Wochenschr., 1899, Nos. 33 and 34. 
very similar to obliteration of the lumen due to the involntion processes which have been mentioned above. The appendix then forms a solid membranous band of uniform thickness or with a few small protrusions. As a rule it is found embedded in peritonitic adhesions.

Sometimes primary slight lesions of the appendix lead to complications, especially if a stricture is present. Thus an accumulation of secretion within the occluded appendicular eavity may take place and give rise to the formation of a cyst. Such cysts occur, varying in size from a cherry to a fist. Guttmann ${ }^{1}$ observed a cyst of the appendix fourteen centimetres (five and a half inches) long and twenty-one centimetres (eight and a quarter inches) wide. The contents of such a cyst are either of a watery mucous character or gelatinous.

If ulceration takes place in the occluded appendicular cavity, it may give rise to the formation of a small abscess (pr-appendix or empyema processus vermiformis). In these cases the purulent process may penetrate the wall of the appendix and lead to perforation. A timely operation in many instances prevents such an outcome.

2. The serere form of appendicitis (appendicitis nlcerosa et gangrenosu, appendicitis perforativa). In this group the bacterial infection is of a much more virulent nature than in the catarrhal form. The inflammation originating in the mucosa of the appendix at once involves all its layers, including the serosa. Necrobiotic processes and formation of pus take place quite early. The peritoneum is also very soon involved, either in the immediate veighborhood of the appendix or in its entirety. Ulcerations and gangrenous processes may lead to the destruction of a

'P. Guttmann: Verhandlungen des Vereins für innere Medicin zu Berlin, 1883-84, p. 301. 
part of the appendis, thus causing perforation, or to a total necrosis of the eutire appendix. As a result of this process the latter may be cast off from the crecum and be found free in the peritoneal cavity or embedded in pus.

The way in which the peritoneum is involved is quite variable. There may be an adhesive type of peritonitis leading to a matted and agglutinated condition of the appendix, or a circumscribed or diffuse peritonitis without adhesions. The coutents of the appendix may be emptied into the abdominal cavity or liemmed in by adhesions. The size, location, and direction of the abscess differ greatly. The location and length of the appendix and the portion perforated play an important part in this respect. In the great majority of cases the abscess is at first intraperitoneal, but very soon extends toward the surface or above or below Poupart's ligament. Again it may penetrate into the bladder, vagina, small intestine, or rectum. In some instances it reaches the diapliragm and from there perforates into the pleural cavity.

In some very grave cases there is no abscess but a ditfuse peritonitis. Here we often meet with a paretic condition of the intestine, the latter being filled with gas; the serous layer is shiny and red, while there is an absence of any exudation. In other cases a small quantity of a purely serous or bloody serous exudation is found. The condition just described may be discorered either in operations undertaken very early or at aintopsies in cases which terminate fatally at the beginning of the disease. In still another group of cases which is a comparatively very small one, the general peritonitis may assume a more protracted and chronic form. In these cases mattings and adhesions are formed over more or less large areas of the abdominal cavity, and in these accumulations of pus may be found. 
Appendicitis due to tuberculosis is of comparatively rare occurrence and shows a great tendency to the formation of fistule. Recently actinomycosis has been found to be the cause of some cases of appendicitis with the formation of abscesses. In these cases the actinomycosis fungi can easily be demonstrated.

Symptomatology.-In describing the symptomatology of appendicitis it will again be best to differentiate the two forms already mentioned above, namely, the catarrhal and the serere form.

1. Catarrhal or endo-appendicitis. An attack of appendicitis is usually characterized by a sudden appearance of pain in the abdominal cavity, which at first may be diffused or in the region of the navel, but very soon is localized in the right iliac region. A moderate rise of temperature is very frequently present. Slight gastric symptoms, nausea, and sometimes vomiting often occur, but are, as a rule, only transient. The pains usually increase in intensity, and the patient assumes a fixed position with the legs flexed. Any change in the position or any movement of the thighs increases the pain. Examination by palpation shows extreme tenderness on pressure of the right iliac region, more especially at McBurney's point, while the rest of the abdomen can be examined by pressure without giving rise to the slightest pain. While the pains are generally continuous, they may show periods of exacerbation. The latter, according to Nothnagel, are most probably due to a spastic contraction of the muscles of the appendix.

The term "appendicular colic" has been given by Talamon to the same condition. Talamon, however, assumed that the colic is always due to an attempt of the appendix to rid itself of a fecal concretion. Inasmuch as operations for appendicitis have often been performed during the at- 
tack of colic and no fecal concretions whatever found in the appendix, and inasmuch as coproliths have been found in cases in which no colic whatever existed, this theory cannot be maintained.

In some cases there is an area of resistance in the right iliac region. If the latter be due to an accumulation of fecal matter in the cæcum, the tumor can be slightly moved and its shape changed by pressure. In a few of the cases of catarrhal appendicitis the resistance is due to an inflammatory swollen (serous) condition of the appendix and of the neighboring organs. In this instance the tumor is not circumscribed but rather diffuse, immovable, and its shape unaffected by pressure.

In comparatively few cases can the appendix be directly palpated. It then appears as an elongated round body of the size of the little finger, and is very painful on pressure. The examination of the appendix itself, whenever this is possible, is certainly of the utmost importance for diagnosis. Edebohls ${ }^{1}$ deserves much credit for having cultivated and perfected the method of examining the appendix by palpation. According to Edebohls, this examination is best done as follows: The patient lies upon his back with the legs comfortably flexed. The physician standing at the patient's right begins to search for the appendix by applying two, three, or four fingers of his right hand, palmar surface downward, almost flatly upon the abdomen at or near the umbilicus; while now he draws the examining fingers over the abdomen in a straight line from the umbilicus to the anterior superior spine of the right ilium, he notes successively the character of the various structures as they come beneath and escape from the fingers passing over them. In doing this the pressure exerted must be

'Edebohls : American Journal of the Medical Sciences, May, 1894. 
strong enough to recognize distinctly along the whole route traversed by the examining fingers the resistant surface of the posterior abdominal wall and of the pelvic brim. Only in this way can we positively feel the normal or slightly enlarged appendix. Pressure short of this must necessarily fail.

R. T. Morris ' suggests for Edebohls' method of palpating the use of three right-hand fingers to feel with and three left-hand fingers placed upon these to press with. The fingers that are to do the feeling are pressed by means of the three others down to the border of the right rectus abdominalis muscle at the level of the navel and slowly drawn toward the examiner. I have found both these methods very useful in detecting the position and size of the appendix.

The temperature is usually but slightly raised, sometimes even normal. The pulse likewise is either normal or but moderately accelerated.

Constipation is often present, but seems to be rather the result of the inflammatory condition of the appendix than its cause, as was formerly beliered. In a comparatively small number of cases diarrhœea is present during the attack of appendicitis.

Course. - An acute attack of catarrhal appendicitis may last from two to three days to two to three weeks. After this variable period of sickness the symptoms either entirely disappear or persist in a slight degree. With regard to the further derelopment the following classes must be distinguished: 1. There may be complete recovery without any further trouble. 2. The patient may entirely recover from the present attack, but have a return of the disease after a variable period of time (from a few weeks, a few

' R. T. Morris: “Lectures on Appendicitis," New York, 1899, p. 45. 
months to a year or two)- "recurrent appendicitis." 3. The symptoms may not completely disappear but may persist for many weeks and the patient may remain in a lingering condition-"subacute or chronic appendicitis."

The first class of perfect recoveries is comparatively small. In this group there is either an obliteration of the appendix or the catarrhal process may have subsided completely without having left behind any lesions. The second class of recurrent appendicitis comprises the majority of the cases. In these a chronic catarrhal condition of the mucosa of the appendix may persist without manifesting symptoms until a new invasion of micro-organisms gives rise to an acute exacerbation of the process, or strictures of the lumen of the appendix may hare formed as a consequence of the acute attack and thus become the cause of renewed disturbances later on. Iu the third category the catarrhal appendicitis has led to severe anatomical lesions. There may be a considerable thickening of the appendix wall including the serosa. The appendicular lumen may show ulcerations, strictures, or bends. There may also be an accumulation of pus (pr-appendix).

2. The severe form of appendicitis (appendicitis suppurativa or perforans). The disease usually begins quite suddenly in the midst of perfect health; rarely it is preceded by slight digestive disturbances. The patient is seized with violent pains in the abdomen. These are felt at first either over the entire abdomen, in the epigastric region, or on the left side of the abdomen, but very soon they settle in the right iliac region. The pains are of an intense character, and occasionally are accompanied by paroxysms during which they are almost unbearable. Any motion increases the pain. The patient lies perfectly motionless and breathes superficially. The appearance is that of a 14 
very sick person, the countenance manifests great suffering and anxiety. The temperature is usually considerably increased and continues so during the first days of the disease. The pulse is accelerated. Occasionally it is of small calibre, easily compressible, and at times irregular. The latter phenomena are found principally in critical conditions. A very frequent pulse and a comparatively low degree of fever are also considered bad omens. There is always complete anorexia and great thirst, the tongue is dry and thickly coated, the bowels, as a rule, are constipated. In rare instances there is diarrhœea. According to Nothnagel, vomiting is present in almost threequarters of the cases. It usually appears right at the commencement of the disease and lasts only a short time. In exceptional iustances it persists for several days. The vomited matter consists of gastric contents, mucus, and bile. In very grave cases it exceptionally assumes a feculent character. The vomiting is occasionally accompanied by hiccoughs. Bcth these phenomena are very annoying and at the same time increase the pain through the motions eroked by them.

In many of the cases, soon after the commencement of the disease a tumor begins to form in the right iliac region. At first a rigidity of the muscles in this region is noted; later on a distinct resistance over an area of egg size may be found. The tumor is either circumscribed and sharply defined, or it is diffuse and connected with the neighboring tissues. The skin over the tumor is as a rule easily movable, while the latter is immovable. The tumor generally consists of a purulent exudation in and around the appendix and congested portions of the intestines, occasionally of the omentum, and of a purulent infiltration of the abdominal wall itself. In some instances 
the size of the tumor is considerably increased by an accumulation of fecal matter in the creum. The tumor may be discovered by palpation and sometimes by percussion. Fluctuation is present only in very extensive abscesses. Its absence does not signify the absence of pus. The resistance as a rule increases either very slowly or quite rapidly. In rare instances, namely in those in which the abscess is surrounded by a firm capsule, it may remain unchanged for a long time. The abscess occasionally involves the muscles and even the skin lying above it. The latter becomes infiltrated and oedematous, and in rare instances the abscess may spontaneously opeu through the skin. Occasionally the resistance disappears entirely when the purulent exudation has descended into the deeper parts. In such an event, by an examination through the rectum, and in females through the vagina, the exndation may be discovered filling Douglas' space.

In cases in which there is an extensive inflammation of the peritoneum accompanied by a considerable quantity of pus, severe pains in urination appear (quite early, after two or three days (Fleischer). On this account the patients are often rather afraid to urinate. In the same cases there may also be paræsthesia and anæsthesia in the limbs, or obstinate erections of the penis, or a drawing up of the right testicle. These symptoms all show that the accumulation of pus presses upon the nerves of the sacral plexus.

The further course of the disease will largely depend upon the way in which the newly formed pus around the appendix acts. Often it leads to a perforation of the appendix. Sometimes the abscess forms adhesions and is encapsuled. Sometimes, again, the abscess penetrates into the peritoneal cavity and gives rise to diffuse septic or fibrino-purulent peritonitis. 
Perforation of the appendix which occurs quite frequently in this class of cases is accompanied, according to Sonnenburg, by the following symptoms: The disease begins with febrile and marked symptoms; violent pains in the abdomen appearing either suddenly or after a short period of slight uneasiness and concentrating very quickly in the right side; romiting accompanied by diarrhœa and in other cases by constipation; small and frequent pulse; fever commencing with chills and quickly rising; pronounced tympanites; general appearance extremely bad; slight eyanosis and perspiration; a distinet area of resistance over or around the affected spot. While all these symptoms are certainly found in cases of perforation of the appendix, they can by no means be absolutely relied upon; for they may exist in the same manner without a perforation taking place, and, on the other hand, the latter event may occur without any of the above-mentioned symptoms being present. For these reasons Boas ' is reluctant to make the diagnosis of perforative appendicitis, and contents himself with determining the presence of purulent appendicitis.

Perforation peritonitis most often appears between the second and fourth days of the disease (Fitz). The danger of a penetration of pus into the free peritoneal cavity lessens with the length of time the disease has lasted, on account of the formation of adhesions. On the other hand, numerous other perilous events may take place. In some cases a few days after the commencement of the disease there is a subsidence of the most important symptoms (pains, fever, etc.), while in others they persist with undiminished severity. Even in the first class, however, the

' J. Boas: "Diagnostik und Therapie der Darmkrankheiten, "Leipzig, 1899. 
amelioration rarely persists, for pretty soon afterward the pains reappear and the fever recurs, and in connection with these symptoms the inflammation increases and the pus augments. Periods of improvement and exacerbation of the coudition may alternate for quite a while until at last either recovery or a fatal issue ensues.

A spontaneous cure or recovery without surgical intervention may occur in one of the following ways:

1. The abscess may become encapsulated, the pus losing its virulence and becoming absorbed. In such an event the tumor disappears and the patient is either definitely or apparently cured; for dangers to life remain after such a cure in consequence of the remnants of the abscess and of the adhesions formed among the intestines. "The occurrence of a sudden bursting of the abscess," using Ewald's words, " hangs like the sword of Damocles over the head of the patient as loug as there is still pus present." In seemingly perfect health a fatal peritonitis may thus occur in patients who had previously suffered from an attack of appendicitis.

2. A cure may be established by the opening of the abscess into adjacent hollow viscera. Thus the abscess may open into the crecum, colon, small intestine, bladder, vagina, or pelvis of the kidney. This favorable issue is, however, rare.

3. The abscess may find its way exterually by rupturing spontaneously through the skin. Sometimes, however, the pus burrows into other organs; thus it may reach the diaphragm (subphrenic abscess), and sometimes eren force its way through into the pleural cavity and perhaps the lungs. But even from these places the pus may be evacuated spontaneously, principally through rupture into a bronchus and its expulsion during a coughing spell.

In a large number of cases peritonitis and septicæmia 
terminate the life of the patient; in others after recovery there are frequently grave recurrences of the disease.

Diagnosis. - Catarrhal appendicitis can be diagnosed if there is a sudden onset of pain in the right abdominal cavity, principally in the region of the appendix, combined usually with a slight rise of temperature and some light gastric symptoms (nausea, anorexia, vomiting). The grave form of the disease or purulent appendicitis shows the same manifestations, only of a much severer type. Besides there are always present signs of serious illness. The patient is very pale and manifests an anxious appearance. Chills are frequently present and the temperature shows a certain irregularity in its course. There may be a marked rise in temperature after it has been quite low or almost uormal for a time.

The presence of a tumor in the right iliac region is of great importance in the diagnosis of appendicitis, although this symptom is frequently absent. In order to recognize the nature of the tumor with regard to its contents, especially whether pus is present or not, Sahli first suggested the use of an exploratory puncture. If pus can be aspirated through the needle, then an abscess is positively present. Although many physicians make use of this method even nowadays, as for instance Leyden, ${ }^{1}$ Nothnagel, Penzoldt, ${ }^{2}$ Fleischer, Boas, and others, most of the surgeons are decidedly opposed to this diagnostic measure (Fowler, Treves, ${ }^{3}$ Sonnenburg, and others). In this country the consensus of opinion is against the nse of exploratory puncture, for its employment adds a new element of

'E. von Leyden : Berl. klin. Wochenschr., 1889, No. 31.

'Penzoldt: "Behandlung der Erkrankungen des Darms." Penzoldt-Stintzing's "Handbuch der speciellen Therapie innerer Krankheiten," Jena, 1896.

3reves: "On Peritonitis." British Medical Journal, 1894. 
danger to the case, while its results, especially if negative, are unreliable.

While appendicitis can usually be diagnosed without difficulty, in some instances its recognition is quite difficult. In cases in which the appendix is abnormally situated, as for instance in the left iliac region or in the upper part of the right abdominal cavity, the diagnosis of appendicitis' will hardly be possible.

Differential Diagnosis. - The following conditions may at times be confounded with appendicitis, namely, biliary, renal, and intestinal colic. The following points will serve as a guide in making a correct diagnosis. In biliary colic the pains are referred by the patient to the right abdominal cavity, radiating to the back and up to the shoulders. Palpation shows a painful area situated immediately below the right margin of the ribs; occasionally jaundice is present. In kidney colic (right side) the pain is felt by the patient in the right lumbar region, radiating toward the bladder. There is generally a frequent desire for micturition and slight burning in the urethra. The urine may show the presence of mucus, sometimes of blood and pus cells. In intestinal colic the pain may be referred to the right iliac region, but, as a rule, it is relieved very soon after the passage of flatus. In contradistinction to these three conditions the pain in appendicitis is referred to the right iliac region, where it remains localized, does not disappear upon passage of flatus, does not radiate to the shoulder and but very rarely to the bladder, while there is also great tenderness and pain upon pressure at McBurney's point. No jaundice is present and the urine is normal.

In women the differential diagnosis between appendicitis and a right-sided salpingitis is not alwars easily made. A thorough examination through the ragina, howerer, will 
in most instances enable us to decide as to the true condition. If the appendix is situated in the sinall pelvis and has given rise to the formation of an abscess in this locality the decision of the question whether the abscess is due to appendicitis or to oöphoritis is extremely difficult and sometimes even impossible. Typhoid fever in exceptional cases may simulate an appendicitis; the presence or absence of Widal's reaction will serve to differentiate the former.

Prognosis. - Catarhal appendicitis affords in most instances a favorable proguosis as regards to life. With reference to complete recovery, however, the outlook is by no means bright, for the liability to recurrence of the disease is very great. Inasmuch as an apparently mild form of appendicitis may all of a sudden change its character and assume alarming features, the prognosis should always be marle with a certain reserve, even in this class.

The puruleut form of appendicitis must be regarded as a very serious disease and gives quite an unfavorable prognosis unless timely surgical intervention is adopted. The intensity of the symptoms in purulent appendicitis is by no means a correct measure of the gravity of the disease. Experience shows that cases with violent symptoms, very high fever, aud intense pains, etc., occasionally recover within a fow days, the pus rupturing into the intestine, while apparently mild cases after a few days of sickness suddenly develop symptoms of a general septic peritonitis with a fatal issue. Diffuse peritonitis is liable to occur between the second and fourth days of sickness, but even later the patient is subjected to numerous risks. Grave complications may suddenly develop even in a patient who is apparently progressing nicely and already convalescent. Thus puralent appendicitis may give rise to pyopneumothorax, empyema, or purulent pericarditis, 
and these complications may result in a fatal issue. The prognosis of perforating appendicitis is decidedly less favorable than that of a simple empyema of the appendix, as in the former septicremia is liable to occur.

After having described the numerous dangers present in the severe form of appendicitis it is consoling to say that spontaneous recoveries are, notwithstanding this, in the majority. With regard to the frequency of spontaneous recoveries Nothnagel gives the following statistics: Among 130 hospital patients he observed 85 complete recoveries, 4 deaths without operation, 30 partial recoveries, and 11 cures after operation. The large number of cases reported by Sahli is also very important in this connection. This author reports the results in 7,213 cases of appendicitis; 473 cases were operated upon, while, 6,740 received only medical treatment. Among the latter 6,194 recovered (91.2 per cent) while 591 ( 8.8 per cent) died. Sahli further states that of the 4,593 cases which had not been operated upon and in which inquiries had been made with regard to recurrence of appendicitis, 3,635 were cured without any recurrence.

Nothnagel says that circumscribed appendicitis is curable in the large majority of cases, and that about eighty per cent recover under simple medical treatment. Among the rest there are still some that can be cured by means of operative procedures. Careful watching of the patient and timely surgical intervention in proper cases may reduce the number of deaths from appendicitis to perhaps five per cent or three per cent. It is, however, impossible entirely to avoid fatal issues, even with the greatest and strictest watchfulness. Aside from accidental complications and from rare cases in which a correct diagnosis is hardly to be made, there remain instances in which 
the peritoneum is diffusely affected quite early without presenting any symptoms. These are the cases which make the prognosis unfavorable and they form the greatest contingent of deaths among patients with appendicitis. The acute septic form with perforation of the appendix is the most dangerous, while the progressive suppurative form is comparatively farorable.

Treatment.-With reference to prophylaxis the swallowing of fruit pits, of very small bones, and coarse, indigestible matter in the food was formerly strictly forbidden. Nowadays, however, we know that the above-named substances play no part whatever in the etiology of appendicitis. Regulation of the bowels or, more practically speaking, correcting constipation has been beliered to be of importance in preventing appendicitis. This maxim can likewise not be maintained on the ground of recent researches. Regularity of the bowels is in itself of importance, and hence it will be advisable to pay attention to this factor. The only means we possess of preventing an attack of appendicitis is the remoral of the appendix. While this suggestion is not generally practicable, for it requires an operation which is not entirely without risk, it may, however, be carried out in cases requiring a laparotomy for other diseases, provided that this additional operation does not demand too much time.

The medical treatrient of appendicitis consists in absolute rest of the entire body, especially of the intestinal tract, and in appropriate diet. The patient must be kept strictly abed from the commencement of the disease until it is entirely over. He should not be permitted to leave the bed for a moment. He must lie perfectly quiet; eren turning from one side to the other should be avoided, or if done, performed with the greatest care. In taking nour- 
ishment the head may be slightly raisea; the urine should be voided in a.glass, and an evacuation of the bowels should take place in a bed-pan. During this act the patient must be forbidden to strain or exert himself in any way. The utensils needed must be handled by the nurse, who must also attend to the cleansing of the patient.

The principle of rest must also be applied with reference to diet. During the first few days of illness there should be either total abstineuce from food (only small quantities of water being given now and then), or liquid food in small portions. Thus strained barley, water, or this with the addition of a little milk, oatmeal water and rice water given in the same way, chicken soup, very weak tea. In the very severe forms of appendicitis, especially when perforation has taken place, or when symptoms of ileus and fecal vomiting are present, absolute abstinence from food and also drink is necessary. In accordance with Penzoldt, Ewald, and Boas, rectal feeding appears to me to be contraindicated in these cases and the only way of supplying the organism with nutritive material is a subcutaneons injection of saline solutions, sugar solntions, and also perhaps small subcutaneous injections of olive oil.

Small pieces of ice may from time to time be given to the patient. He must, however, keep the ice in his mouth until it melts before swallowing. This often alleviates the nausea and retching. The first two or three days of sickness being over, the patient may be allowed to have milk, an egg beaten up in bouillon or milk, in addition to the abovenamed food. The diet should be kept up in this way until the pains and ferer have entirely disappeared. At this period soft-boiled eggs, crackers, small portions of meat (squab) or chopped beef may be giren, and still later mashed potatoes, bread and butter, and light vegetables added. 
Medicaments. The use of cathartics is mentioned here only in order to condemn it. Even injections into the bowels should not be administered too frequently nor in large quantities. A small enema of one-half to one pint of water or one-half pint of olive oil may occasionally be given.

The remedy par excellence in the treatment of appendicitis is opinm. Its use was originally recommended by English physicians (Graves, Stokes) and later by French clinicians (Petriquin, Grisolle); in Germany this remedy found a fervent advocate in Volz and in America in Alonzo Clark. During the last decade the administration of opinm has met with great opposition especially on the part of many surgeons. Their reasous against the use of this remedy are, first, that opium masks the true picture of the disease, and secondly, that it gives rise to paralysis of the intestines.

Some of the foremost clinicians, Nothnagel, Penzoldt, Ewald, Sahli, Boas, and others, are even nowadays enthusiastic admirers of the opium treatment. The principal element of importance of opinm as a remedy is its action in lessening or arresting the peristalsis of the intestine, and besides in alleviating paiu. I myself have always used and still nse the opium treatment with great satisfaction. It is of course understood that the opium should be given only in sufficient amount to allay the pain, while excessive doses should be avoided. As soon as the active stage of the disease is passed, the opium must be entirely discontinued. The best way of administering it is that suggested by Sahli. Ten or fifteen drops of tincture of opium are at first given every hour until there is a decided subsidence of the pain. Then five to six drops are given every two or three hours until the pains disappear completely. As soon as there is an exacerbation another large dose is administered, but if the patient is entirely 
free from pain no opium is given. If the administration of the drug excites nausea or vomiting, it may be given in the form of a suppository:

R Extr. opii........................ 0.05

Ol. theobrom..................... 1.00

I. f. supp. One suppository every four hours until subsidence of pain.

Or belladonna extract, $0.005-0.01 \mathrm{gm}$., may be added to the opium in the same suppository.

Boas recommends the administration of opium subcutaneously. (Extr. opii aquosi sterilis. 0.3 to 10.0 water; 1 Pravaz syringe $[1 \mathrm{gm}$.] three times daily.)

In cases in which the pains are very intense and a quick action is desired, morphine may be administered subcutaneously in doses of gr. $\frac{1}{6}$ to $\frac{1}{4}$. The action of this remedy is, however, not so satisfactory as that of opium, as it has but a very slight influence in diminishing the peristalsis. When morphine is used, opium should be given in addition.

Poultices. The application of ice over the painful area is often beneficial at the beginuing of the disease, especially if the temperature is cuite high and symptoms of peritoneal irritation are present. If the patient, however, complains of great discomfort from the application of ice, it must be discontinued. In the latter instance a cold Priessuitz poultice may be tried. Cases not accompanied by high fever often derive great relief from the application of a hot-water bag or plain warm poultices. The latter are especially to be recommended in that form of appendicitis which is called appendicular colic of Talamon.

Surgical Treatment.-The question of operation in appendicitis is a very live one nowadays and is being everywhere discussed. The medical profession has not yet come to a unanimous conclusion in regard to it. Surgical treat- 
ment of appendicitis originated in this country, Dr. Reginald Fitz of Boston having done the first operation for this purpose, and it has been practised and perfected here more than anywhere else. It is therefore quite natural that we find many more advocates of surgical intervention in America than abroad. As a general rule the majority of surgreons frequently recommend operative intervention, while the larger number of physicians reserve the surgical treatment only for a small number of grave cases of appendicitis.

Fowler, Morris, Beck, Deaver, Murphy, and others in this country and Legueu ' in France urge surgical treatment in every case of appendicitis. Legueu says: "Appendicitis belongs to surgery." . . . "There is no medical treatment of appendicitis." . . " Every appendicitis must be operated early." C. Beck ${ }^{2}$ expresses himself in the following manner: "No matter how mild the clinical picture of appendicitis appears, eren if it promises a quick temporary recovery, the operation is always justified. Inasmuch as the gravity of infection cau never be estimated at the beginning, it appears wiser to look upon every case of appendicitis as serious. Of two evils one should choose the lesser, and the lesser one here means operation." In his article Beck makes the two following assertions: "1. Appendicitis is a surgical disease and should be treated surgically as soon as diagnosed. 2. So long as no physician is able to estimate the gravity of the bacterial infection at the commencement of the disease or to foresee the course which the appendicitis will pursue, whether mild or grave, the safest treatment consists in the early removal of the appendix."

'Felix Legueu: "Traitment de l'Appendicite." Suite de Mono. graphies Cliniques, 1899, No. 80.

2C. Beck : “A Appendicitis." Volkmann's Sammlung klinischer Vorträge, No. 221, Sept., 1898. 
Many surgeons, even in this country, however, do not take so radical a view as the above writers. Thus Willy Meyer, ${ }^{1}$ Charles McBurney, W. T. Bull, A. J. McCosh and F. Hawkes, ${ }^{2}$ and others do not recommend the early operation in milder forms of appendicitis. McCosh and Hawkes express themselves in the following manner with regard to the necessity of operative interference: "When the presence of pus is assured, the sooner operation is done the better. Also there are cases which begin and continue for twentyfour or forty-eight hours with such severity that a judicious mind must conclude that operation is demanded. So in the chronic and relapsing cases where the symptoms have continued for months with such severity and have recurred so frequently as to subject the patient to a life of semi-invalidism, no wise surgeon can counsel any other plan of treatment than removal of the diseased appendix. Likewise when the patient has suffered from three or more attacks the offending organ should be removed, for other attacks will in all probability follow. The same indication for operation also exists in our opinion if a patient has suffered from two attacks within a year or even two years." ... "The view which we take is that operation is not necessary in every case of appendicitis. We believe that not infrequently patients recover, and recover permanently, from one attack of appendicitis, and that in a certain number of cases, provided a careful watch is kept, operation is not necessary. On the other hand, we acknowledge that many cases which did not appear to be serious have been allowed to die when they might have been saved by operation."

! Willy Meyer : "When Shall we Operate for Appendicitis?" Medical Record, February 29. 1896.

${ }^{2}$ A. J. McCosh and F. Hawkes: "The Surgical Treatment for Appendicitis." The American Journal of the Medical Sciences, May, 1897. 
Willy Meyer, who was among the first to recommend the removal of the appendix during the free interval, comes to the following conclusions in his paper already mentioned: " 1 . In case of diffuse perforative appendicitis the operation must always be done at once. 2. In cases of acute appendicitis the patient always needs careful observation. If the pulse goes above 116 and 120 and has a teudency to stay there, the indication for an operation is given. In cases of doubt the operation is better than waiting. In cases of subacute attacks of appendicitis, also after the first severe attack from which the patient recovers without immediate operation, the appendix should be removed. The appendix once inflamed has to be looked upon as a diseased organ which is very apt to give repeated and more serious, even fatal, trouble in the future."

Among the German surgeons Somnenburg, and especially Riedel, ' are advocates of early surgical intervention in the grave forms of appendicitis. Riedel says: "As soon as the temperature reaches $101^{\circ}$, the pulse 100 , the immediate removal of the appendix is indicated. . . A tumor which has dereloped, accompanied by fever and an acceleration of the pulse, is always an indication for immediate operation." R. Stein ${ }^{2}$ and Henry J. Wolf, ${ }^{3}$ in papers read quite recently before the German Medical Society of New York, urged early surgical intervention in all the graver forms of appendicitis.

With Penzoldt, Nothnagel, Ewald, Boas, and others I would give the following indications for surgical intervention in this disease:

\footnotetext{
' Riedel: "Ueber die sog. Fruhhoperation bei Appendicitis puru lenta resp. gangraenosa." Berliner klinische Wochenschrift, 1899, 33 und 34.

? R. Stein : Deutschē med. Wochenschr., 1899, p. 440.

${ }^{3}$ H. J. Wolf : New Yorker medicinische Monatsschrift, 1899.
} 
1. Diffuse peritonitis in consequence of perforation of the appendix demands immediate operation. As a rule the following symptoms will be found: Sunken and drawn features, cyanosis, a small and very frequent pulse, an increase of the painful area, often also a bloated condition of the abdomen.

2. Whenever an appendicular abscess showing fluctuation is present, an operation should be performed.

3. If the protracted course of the disease points to the existence of an abscess, giving rise to slight septic symptoms, an operation should be undertaken.

While in these three groups there can be no hesitation in recommending the operation, in the following groups the necessity of surgical intervention must be considered and decided in each individual case.

4. (a) If the rational treatment does not produce any improvement in the course of three to five days, the symptoms persisting in undiminished severity or becoming even more pronounced, an operation may be resorted to. (b) A sudden rise of temperature lasting over twenty-four hours, after the first few days of sickness, is also an indication for operation. (c) A very frequent pulse, not corresponding to the degree of fever, is another symptom which justifies the consideration of an operation. (d) If the tumor continues to increase in size after the fifth day of sickness, an operative treatment should be considered.

5. The removal of the appendix should be undertaken: (a) In all cases of appendicitis in which after recovery the pain in the right iliac region persists for a long time (several months); $(b)$ in recurrent appendicitis if the attacks have been quite severe or if they have followed each other at short intervals. 


\section{CHAPTER IX. \\ INTESTINAL OBSTRUCTION.}

(Acute and Chronic.)

Introductory Remarks.-By intestinal obstruction is understood a great variety of conditions which, although unlike in character, have yet the common feature of mechanically causing an obstruction to the passage of contents along the intestine. Leichtenstern ' distinguishes the following three groups with regard to the causation of the intestinal obstruction:

1. Occlusion' due to pressure from without or compression of the intestinal lumen in the full sense of the word. To this group belong incarcerations of the intestines in apertures, in slits, and in hernial openings; strangulation by psendo-ligaments, the vermiform process, and diverticula; compression by tumors, by the mesentery, or by displaced abdominal organs. Rotations of the intestinal tube around its axis (torsions) and formation of knots also belong to this category.

2. Occlusion from within the intestinal lumen (obturation). The obturation may be produced either by gall stones, enteroliths, foreign bodies, hardened fecal masses, or by neoplasms of considerable size, especially polypi. Intussusception (involution of one coil of the bowel into another) also belongs to this class.

${ }^{1}$ Leichtenstern: "Verengerungen, Verschliessungen und Lagever-" änderungen des Darms." Zlemssen's “Handbuch der speciellen Patho. logie und Therapie," Bd. vii., Leipzig, 1878. 
3. Occlusions which originate from factors within the intestinal wall and causing narrowing of the lumen either directly or indirectly. Constriction may occur either in circular form (strictures) or as a result of flexions. Obstructions developing after chronic peritonitis, distortions, and angular bends of the intestine, cicatricial stenoses as well as those produced by neoplasms, belong to this class.

Notwithstanding the diversity and great multiplicity of the anatomical factors causing stenoses and obstructions of the intestines, the clinical picture and the consecutive lesions which they evoke greatly resemble each other. It will therefore perhaps be practical to give first the clinical picture of complete obstruction of the bowels (ileus) and of stenosis of the intestine, and then to discuss the different anatomical causes and also the differential diagnosis.

\section{ACUTE INTESTINAL OBSTRUCTION.}

\section{Synonyms.-Ileus, miserere, passio iliaca.}

Definition.-An acute stoppage of the passage of the intestinal contents. This may be caused either by a mechanical occlusion at a certain part of the intestinal canal (mechanical ileus) or by an entire absence of motor power in a portion of the bowel (dynamic or paralytic ileus) or sometimes by both (mechano-dynamic ileus).

Etiology.-The etiology of ileus is quite complicated, and it will be best to analyze separately the different factors producing it.

Compression of the Intestines. Compression of the intestines can occur: (1) by strangulation through adhesions, bends or pseudo-ligaments, by Meckel's diverticulum, by normal structures abnormally attrched, by slits and aper- 
tures in the mesentery and omentum, and by incarcerations into herniæ; (2) by torsions (volvulus); and (3) by tumors from without.

The primary factor in producing isolated adhesions (bands or psendo-ligaments) is a preceding localized peritonitis. In some cases these bands may have been congenital and due to intra-uterine peritonitis. The band may have the form of a firm fibrous cord or it may be very slender and may appear as a tough, rigid thread. Occasionally it may be of comparatively large size. Seldom the constricting ligament has the appearance of an actual band, having a width of half an inch or more.

The strangulation of the intestine by an isolated peritoneal adhesion takes place in two ways: first, the intestine may be strangulated under the band as beneath a shallow and narrow arch; secondly, it may become snared and constricted by a noose or knot formed by the false ligament itself. Strangulation from bands occurs when these are comparatively short and tightly stretched over a firm surface. The arch beneath which the implicated bowel passes is usually large enough to admit one to three fingers. Strangulation by a noose or knot requires the presence of a long false ligament which must lie loose and free in the abdominal cavity, being attached only at its two ends. The most common way in which a coil of intestine becomes snared is where a lax band forms a ring or spiral between its fixed points. Through this ring a loop of the small intestine slips; the protrusion becoming larger the implicated coil cannot free itself from the noose and is strangulated. Strangulation by the formation of a knot is described by Leichtenstern in the following manner: "There are several kinds of this knotting. The most frequent is the following: A long and loose ligament is fastened at one end to 
a loop of the small intestine, and hangs in the form of a simple coil (Fig. 29); if the top of the intestinal loop passes directly through the coil a simple knot is formed about the piece of the intestine, as is shown in Fig. 30. It is evident that the same result can be produced by the
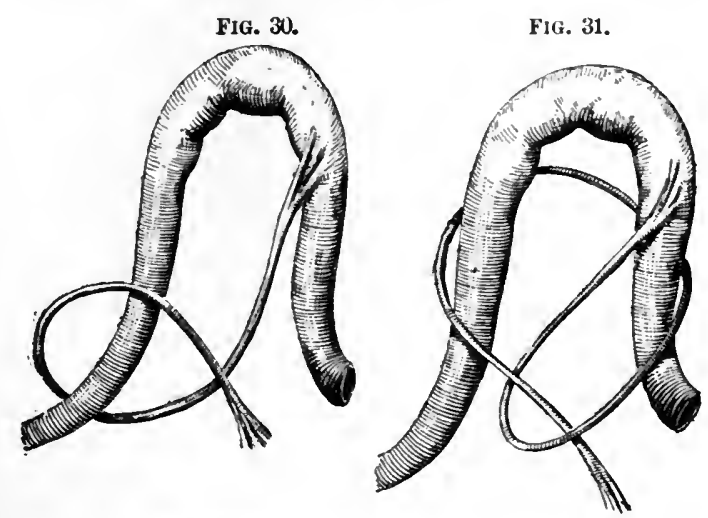

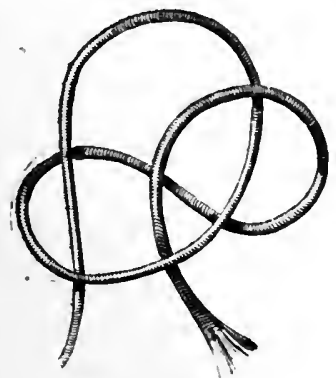

FIG. 32 .

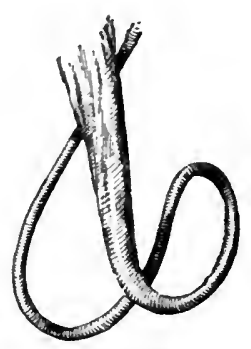

FIG. 29.

Figs. 29-32.-Types of Constricting Peritonitic Bands. (After Leichtenstern and Treves.)

coil being drawn over the top of and around the intestinal loop. Another and rarer form of knot is produced as follows: A long and perfectly loose false ligament forms a simple coil between its points of attachment. If now one leg of the so-called primary noose passes through it we have a knot like that shown in Fig. 31, and if now the 
intestinal loop passes directly through (Fig. 32), it becomes firmly caught and strangulated. A common characteristic of all described knots is that when the strangulated intestine is freed, the ligament can immediately be drawn out straight."

Strangulation by Meckel's Diverticulum. Meckel's diverticulum is due to the persistence or incomplete obliteratiou of the vitelline duct. Most commonly it exists as a blind tube, given off from the ileum. Its length is about three inches. As a rule, it is cylindrical in shape, with a conical extremity. Occasionally it presents a globular shape and is then called "clubbed." Meckel's diverticle is always single and is attached to the ileum one to three feet above the ileo-crecal valve. As a rule, the end of the diverticulum is free. In some instances it is attached to the umbilicus or to the abdominal wall. Sometimes the end attached to the abdominal parietes may give way and form fresh adhesions with some points of the peritoneal surface. The latter occurrence is of great importance with reference to strangulation of the intestine, which frequently takes place under these conditions. By means of the new adhesion of the diverticulum a loop is formed in which some portion of the intestine is liable to engage. Another possibility for strangulation by the diverticulum is afforded when its end is free and club-shaped. The diverticulum forms a ring into which its own free end projects. A loop) of the intestine entering the centre of this ring may push the clubbed end of the process before it and so tie the knot, thus leading to obstruction. Again the diverticulum may surround the pedicle of an intestinal loop in such a way as to encircle it with a single knot (see Figs. 33, 34, 35).

In a similar manner as Meckel's diverticulum some normal structures may act when they are abnormally attached. 
Thus the rermiform appendix may become adherent to some point of the neighboring peritoneum and so form an arch under which a loop of the intestine may be strangulated. The Fallopian tube may likewise become adherent to the adjacent peritoneum situated in the iliac fossa and thus form an arch into which a portion of the intestine may slip and become incarcerated. Other internal organs abnormally attached may form similar traps for intestinal strangulation.

Of great clinical importance is FIG. 33. 1 FIG. 34.
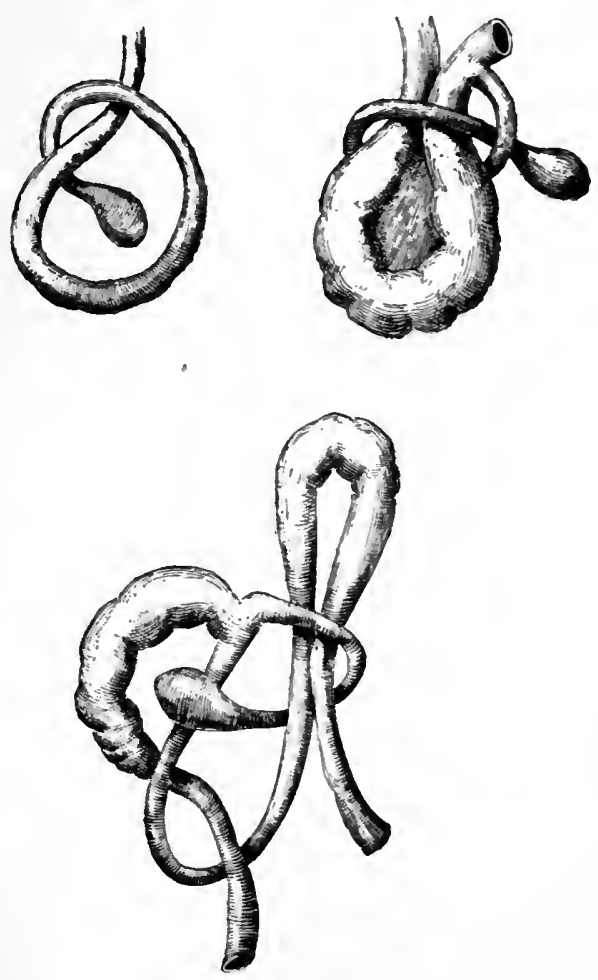

FIG. 35 .

FIGS. 33-35.-Knotting of a Meckel's Direrticulum which has a Button-like Swelling of its Extremity. (Treves.) the strangulation of the intestine in slits and apertures of the mesentery or omentum. These may be either congenital or of traumatic origin. Similar to the action of slits in the production of strangulation are also the various intermal heruia (hernia duodeno-jejunalis, hernia retroperitonealis anterior, 
hervia intrasigmoida, hernia bursæ omentalis, formed by the foramen of Winslow, diaphragmatic hernia).

In all these cases the mechanism of the obstruction is as follows: A coil of gut may be driven with sudden severe force beneath the band or through an aperture and become practically strangulated at once, as is often the case in strangulated hernia. There being no natural force to drive the coil out of its place of imprisoument, it remains firmly gripped. - In other cases the involved intestine may not be strangulated at first, but the band pressing upon the mesenteric vessels produces a congestion in the implicated coils, which become engorged and distended by an increased accumulation of gas, and thus complete strangulation is the result. In other cases, again, the final cause of a strangulation is a twisting of the bowel. All the varieties of intestinal strangulation just mentioned occur in the small intestine, the lower portion of the ileum being principally affected, less frequently its upper portion or the jejunum.

The occlusion may in some cases be due to kinking of the intestine through a band attached to the bowel and dragging upon it. Adhesions may also obstruct the bowel, compressing its lumen. This oceurs when false membranes are situated around the bowel and have undergone shrinking. They then compress the intestine seriously and narrow its lumen. The same process of shrinking may also effect an obstruction of the bowel if it takes place in the mesentery after inflammation.

Volvulus. By the term volvulus is understood an obstruction of the bowel by a twist about its mesentery, or its own axis, or the intertwining of an intestinal coil within another. Twisting of the bowel occurs most often in the sigmoid flexure. The usual cause of this trouble is chronic consti- 
pation, for in this condition the flexure is more or less constantly distended. Its walls become partly paralyzed and hang down into the pelvis, like an inert heavy mass, being filled with fecal matter. Traction is thereby exerted upon the mesocolon and a loop is soon formed. A twisting of the latter is brought about either by some displacement of the bowel or by a sudden change in the position of the body. The ascending colon, creum, and the small intestine may also be affected in the same manner, although less frequently. Intertwining of the intestine is here more often met with.

Obturations of the Intestine. Intestinal occlusion often takes place in consequence of obturation of the lumen of the gut through foreign bodies lodging therein. Accumulations of fecal matter may give rise to such an occurrence. The hard fecal tumor is then situated either in the cæcum or in the colic or sigmoid flexures. In these cases chronic constipation has existed for a long time.

Gall stones, although rarely, give rise to intestinal occlusion. In order to do this they must be of considerable size. "The puzzle as to how the camel could go through the eye of the needle, i.e., how these enormous gall stones could reach the bowel, has been solved, by the assumption on fair evidence that an ulcerative process opens the way from the gall bladder to the bowel, though doubtless very large stones occasionally find their passage through the ducts" (E. D. Ferguson ').

In a similar manner enteroliths may also cause obstruction of the bowel. This happens especially if an enterolith situated in an intestinal diverticulum has been dislodged and found its way into the canal of the gut.

${ }^{1}$ E. D. Ferguson: Transactions of the New York State Medical Association, 1898, p. 233. 
Foreign bodies which have been accidentally or intentionally swallowed may under favorable conditions reach some part of the bowel and here obstruct the lumen. This will occur if the foreign body is of considerable size, or if it is not smooth but provided with sharp points. The latter catch in a fold of mucous membrane and prevent its further passage. The most varied substances have thus been found to be the cause of intestinal obstruction: marbles, stones, coins, glass stoppers, corks, spoons, knives, forks, keys, needles, pins, buttons, false teeth with the plate. Kernels of fruit like cherries, prunes, etc., may accumulate in the bowel and by means of fecal matter be kept together, forming a large conglomeration, completely obstructing the canal.

Recently Murphy's button has also been found in a few instances to cause obstruction of the bowel.

Intestinal parasites (tapeworms, ascaris lumbricoides), if present in large numbers, may also form a mass obstructing the canal. This occurs especially after a vermifuge has been administered and the dead parasites have remained within the canal.

Similar to the action of foreign bodies are also tumors (polypi, fibroma, myoma, etc.) connected by a pedicle with the intestinal wall, filling up its lumen.

Intussusception. Intussusception or invagination means the prolapse of one part of the intestine into the lumen of an immediately adjoining part. An intussusception shows in a vertical section six layers of intestine, three on either side of the central canal, which are more or less parallel to one another. The arrangement of the layers is such that mucous membrane is in contact with mucous membrane, and peritoneum with peritoneum. On transverse section the invaginated mass shows three concentric rings of bowel. 
The external of the three layers is çalled the intussuscipiens, the sheath, or the receiving layer. The innermost cylinder is called the entering layer and the middle one the returning layer. The latter two together form the intussusceptum. The neck of the intussusceptum is at its upper part where the returning layer joins the sheath.

In case the intussusception lasts for some time the serous surfaces of the gut touching each other may become glued together and ultimately adherent. This will prevent the disengagement of the invaginated portion, while its further passage into the other bowel will not be interfered with. The mesentery always participates in the invagination and becomes more or less compressed and wedged in by the sheath. The whole mass of a simple intussusception may in its turn become invaginated and give five instead of three coats, or eren seren if the process is repeated, so that the upper edge of the intussuscipiens is rolled over like a cuff. These double and triple intussusceptions are comparatively rare.

With regard to the mechanism of intussusception Nothnagel's experiments on animals hare proven of greatest value. According to this writer intussusception may be due either to a localized spastic contraction of a portion of the bowel or to a total paralysis. The normal gut immediately below the contracted part slips upward to a slight extent over this strongly contracted and greatly narrowed portion, and invagination is thus produced. Again if a segment of the bowel is paralyzed, the gut lying immediately below it, on contraction will slip into the paralyzed portion and thus an invagination may arise.

Intussusception may take place at auy point within the entire small and large intestiues. Over fifty per cent of the cases consist of the invagination of the ileum into the 
colon. With regard to the remote cause of intussusception Treves ${ }^{1}$ has examined a number of reported cases and found it in one hundred examples of intussusception distributed as follows:

1. No evident exciting cause .................62 per cent.

2. Diarrhœa, dysentery, enteritis, marked irregularity of the bowels..............................

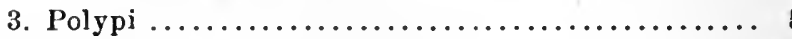

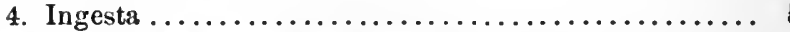

5. Injuries and exposure to cold...................

6. Certain acute and chronic ailments which may or may not have had a concern in the etiology, such as typhoid fever, whooping-cough, measles, scarlet fever, smallpox, cholera, and hernia; with these may be included pregnancy and labor.......... 15

Total

$\begin{array}{ll}8 & " \\ 5 & " \\ 5 & 4 \\ 5 & 4\end{array}$

This clinical form of intussusception must not be confounded with agonal intussusception, which, as the term indicates, occurs shortly before death and is purely of anatomical importance. The agonal form of intussusception is sometimes found multiple and is met with frequently at autopsies of children who have died from affections of the brain.

Pathological Changes. - The lesions which are encountered in acute ileus, no matter what be its origin, are the following: The intestinal coils above the occluded part of the bowels present a quite different appearance from those below. The former are distended, filled with gas and illsmelling feculent contents; and this ectatic condition is the more pronounced the nearer they are situated to the occluded part. If the occlusion lies in the jejunum or ileum, the distention will involve the entire upper portion of the small intestine and also the stomach. If, however, the stoppage is situated within the colon, the dilatation

${ }^{1}$ Treves : "Intestinal Obstruction," p. 211. 
will at first occupy that portion of the colon situated between the ileocecal valve and the obtruded spot, while the small intestine may remain unchanged, the ileocæcal valve acting in its usual way and thus preventing an overflow of the contents of the colon into the small intestine. Under such circumstances the dilated portion of the colon may attain considerable size, resembling almost the stomach. After the condition has lasted a few days, howerer, the ileocrcal ralve ceases to functionate and now the contents of the colon orerflow the small intestiue and the stomach and these organs become also orerfilled and distended. The portion of the intestine situated below the occlusion is empty and contracted.

The intestinal coils above the occluded spot are usually engaged in very active peristaltic movements, which represent an attempt of nature to orercome the obstacle. After these peristaltic motious have lasted a few days, a paraly tic state of the intestines superrenes.

The intestinal mucosa situated near the occlusion is subjected to great mechanical and chemical irritations due to the constant presence of considerable amounts of decomposed material, and thus grows intensely inflamed. Often ulcers develop which may penetrate the wall of the bowel and cause fatal peritonitis. In rare instances after such a perforation, adhesion to neighboring intestinal coils may occur and give rise to fecal abscesses and abnormal communications between different intestiual segments. By means of a similar process an opening may be established between the intestiue and the abdominal walls in such a manner that the fecal matter finds an exit here (anus præternaturalis).

Localized or general peritonitis is thus often present in cases of intestinal obstruction. Serous, bloody, or puru- 
lent exudation is frequently found in the abdominal cavity. The anatomical lesions are most pronounced in the immediate vicinity of the occluded intestine. This is due not only to the stoppage of the intestinal contents but also to interference with the circulation of the gut produced by the same factors which have caused the obstruction. Numerous large and small mesenteric veins become compressed, thus causing congestion and hemorrhages. The intestinal walls appear infiltrated with blood, showing ecchymoses at various places, and may even appear dark red. In the neighborhood of the occlusion the intestine may be covered with black curdled blood in the form of a membrane. Its walls become brittle and gangrenous.

Symptomatology.-The symptoms of acute intestinal obstruction appear either suddenly or after slight disturbances have existed for a few days, as for instance diarrhœa, constipation, feeling of uneasiness. In some instances the history of an exciting cause is given. Thus a severe blow on the abdomen, violent bodily exertion, a cold, a too copious meal, or a strong laxative.

The patients are first seized with violent abdominal pains, sometimes of a crampy character. The pain may be felt at first at a certain definite spot within the abdomen, while later it becomes more diffuse. In other instances the patient is unable to localize the pains distinctly. Occasionally the area around the navel is given as the seat of the pains, while in other cases they are referred to the entire abdomen. The pain usually exists uninterruptedly, though it may show exacerbations from time to time. Soon after the occurrence of these colicky pains eructations of gas and then vomiting appear. At the beginning gastric contents are ejected, later bile, and finally offensive feculent material is brought up. The latter usu- 
ally has a yellowish-brown color, is liquid, and contains only very fine, small, solid particles suspended in the fluid. At this period the eructated gases have a fetid odor and hiccough almost constantly distresses the patient. After the act of romiting the patient may feel somewhat relieved for a short while, but soon there is a return of the severe symptoms.

Almost simultaneously with vomiting, meteorism of the abdomen ensues. The passage from the rectum is entirely stopped and there is no evacuation either of fecal matter or of flatus. The meteorism may involve either a certain region of the abdomen or the entire cavity. The tympanites gradually increases and a feeling of tension becomes more and more pronounced. The diaphragm is soon pushed upward by intestinal coils filled with gas in such a manner that the liver dulness may be absent from the entire right thoracic cavity. Dyspnoea supervenes; the breathing becomes accelerated and superficial, assuming the thoracic type. The pulse is small and frequent. The extremities are cold, the skin is covered with perspiration, the face is pale, bearing the expression of utmost anguish, the eyes are sunken, dryness of the throat and extreme thirst exist, and the patient is barely able to use his voice. These extremely painful and tormenting symptoms persist and the patient succumbs-unless there is a change in the course of the disease-remaining conscions until the end.

After having given a general description of the clinical picture of ileus it will not be amiss to discuss each symptom separately.

1. Pains. Pain, the most constant and conspicuous symptom of intestinal obstruction, depends upon several conditions. It is usually due, first, to the injury inflicted on the peritoneum and the intestival walls in consequence 
of the strangulation; secondly, especially at a somewhat later period, to the tumultuous and increased irregular peristaltic movement of the intestiues. These movements above the site of obstruction are of a very intense character and produce "colic" as well as exacerbations of the pains which occur at certain intervals. The intensity of the pain depends upon the degree of excitability of the individual, upon the state of the sensorium, upon the extent of the intestine and peritoneum involved, and upon the severity of the occluding lesion and the rapidity of its occurrence. Later on the pain is influenced by the distention of the gut and by the presence or absence of peritonitis.

At the commencement of the disease the pain is frequently not aggravated and sometimes relieved by pressure. Later, however, the pain is considerably increased by even slight pressure, the cause of this being the presence of peritonitis.

According to Treves, ${ }^{1}$ the pain is constant, although liable to periodical exacerbations in cases of complete obstruction. In cases in which the obstruction is but partial the pain is distinctly intermittent, and the patient experiences intervals between attacks of pains during which he is free from suffering. The pain as a rule grows more intense with the progress of the disease. There may be, however, a diminution in the severity of the pain for a short period before a fatal issue, caused by a collapse, paralysis of the intestine, rupture or perforation of the bowel, or by a diminished activity of the sensorium.

Treves has pointed out that no matter in what part of the small intestine the obstruction is situated, the pain arising therefrom is usually referred to the region of the umbilicus.

'F. Treves: "Intestinal Obstruction," Philadelphia, 1884. 
If the obstruction is localized in the large bowel, then the pain may be experienced, especially at the beginning of the disease, at the seat of the lesion; later, however, the pain may assume a more diffused character or may be falt at other regions of the abdomen. This is the reason why only the initial pain is of some diagnostic significance with regard to the seat of the lesion.

2. Vomiting. Vomiting is almost always present. At the beginning of the disease it is of reflex origin due to the irritation of the peritoneum; later on it must be ascribed principally to the irregular, strong, peristaltic contractions of the intestines. The appearance of fecal romiting was believed by the old writers to be a sign that the obstruction was situated in the large bowel. Nowadays, however, it is generally known that this symptom is often present in cases in which the obstruction is situated in the ileum or even in the jejunum. The reason of absence of putrefactive processes in the intestinal contents normally is the rapidity with which they are moved farther on along the canal until they reach the large bowel. In obstruction, however, the peristaltic contractions are much slower and thus putrefactive processes develop even in the small bowel.

In order to explain the mechanism of stercoraceous vomiting a reversed peristaltic or antiperistaltic motion of the intestines was formerly assumed. Of late, however, the mechanism of fecal romiting as expounded by Haguenot ' as early as 1713, is now generally accepted. According to this author, stercoraceous vomiting takes place in the following manner: Above the occluded intestine there is an accumulation of more or less liquid intestinal contents in

1 Haguenot: "Mémoire sur les Mouvernents des Intestius dans la Passion Iliaque." Histoire de l'Académie Royale des Sciences, Paris, 1713. 
considerable quantity; the bowels being distended with large amounts of gas are under constant pressure, which is increased after each inspiration and especially after energetic contraction of the abdominal muscles, occurring for instance during the act of vomiting. Under the influence of pressure the stagnant liquid contents are regurgitated from above the occluded spot into places in which there is less resistance and thus reach the duodenum and the stomach. Here they irritate the mucous membrane and cause vomiting.

This theory is perfectly in accord with the circumstance that in stercoraceous vomiting mostly liquid or sometimes semi-liquid contents are evacuated, but never solid fecal matter; for even in obstruction of the colon the fluid will be moved farther upward while solid particles will remain in the lower portion of the bowel. Vomiting of formed fecal matter is a rery rare occurrence, and must be ascribed to an existing fistulous opening between the colon and stomach.

3. Constipation. Constipation almost always exists and is very obstinate. After injections, very rarely spontaneously, there may be a slight movement of the bowel consisting of the fecal matter lodged below the occluded spot. In some rare instances a catarrhal condition may exist in the segment of the bowel below the obstruction, and the patient then may rather have diarrhoea combined with tenesmus. Of greater significance than the absence of stools is the inability to pass wind through the anus. The passage of flatus is a sure sign that the permeability of the intestine has been re-established.

4. Meteorism. Meteorism is the result of increased formation of gas developing in consequence of putrefactive processes as well as of diminished absorption. According to Zuntz, the absorption of intestinal gases into the blood 
takes place only when the circulation is in good working order. Meteorism thus indirectly points to a disturbed circulation which is often found in cases of incarcerations. If meteorism is absent the absorption of gases must be assumed to take place as rapidly as their formation. Meteorism may be at first present at a certain circumscribed spot of the abdomen and later become more diffuse. If the place at which it first appears can be distinctly defined, this is of diagnostic importance with regard to the location of the occlusion.

If the occlusion is in the large bowel the portion situated between it and the ileocæcal ralve will become considerably distended with gas. Thus a protrusion of the right side of the abdomen will be noticed when the obstruction is at the right flexure. If the obstacle is situated in the rectum there is at first a protrusion of the left side of the abdomen and later the tympanites will involve the portion of the abdomen situated above the narel (course of the transverse colon). In some instances, however, obstruction of the rectum may be acompanied by more or less general meteorism. This is especially the case after the disease has lasted some time; for then, as a rule, the resistance of the ileocæcal valve is overcome by the gas pressure and it remains more or less patent in such a way that the gases easily penetrate the small intestine.

In occlusions affecting the duodenum or the upper part of the jejunum the meteorism as a rule involves the upper half of the abdomen, and remains confined to this area. After vomiting there is usually a perceptible decrease of the protrusion for a short while.

If the meteorism has lasted for some time and is intense, the abdomen assumes a barrel shape. This is especially found in cases in which the distended intestinal coils 
are already paralyzed. The accumulation of gas can now go on without encountering much resistance and thus do great harm. The diaphragm is then pushed upward. The lungs as well as the heart become compressed. Stomach, liver, and bladder are compressed by the intestinal coils filled with gas lying upon them. 'In a similar manner the large veins (vena cava, vena portre, etc.) are subjected to the same disturbance. Thus the function of many important vital organs is interfered with and impaired to such a degree, if this condition persists, that a fatal issue may occur.

5. Collapse. The diverse symptoms of shock which appear in a marked degree in cases of ileus must be ascribed to the sudden damage inflicted upon the peritoneum and intestinal wall by the strangulating agent. The mechanical irritation involves first the splanchnic nerves, and through them the circulatory apparatus. As a consequence there are a lowering of the temperature of the surface, cold sweats, lividity of the extremities, anæmia of the brain, and a small and rapid pulse. The degree of the collapse depends upon the disposition of the patient, upon the suddenness of the strangulation, and upon the amount of peritoneum or of intestine iuvolved in the lesion.

The gravest amount of shock is met with in cases in which a considerable segment of the intestine is suddenly strangulated and an injury thus abruptly inflicted upon an extensive nerve area. As a rule, the shock met with in cases of obstruction of the small intestine is much more pronounced than in cases in which the obstruction is situated in the large bowel. The reason for this is the greater supply of nerves and the greater activity of the small intestine as compared with the large bowel. The nerves of the small intestine are also more directly associated with the great sympathetic ganglia of the abdomen. 
6. The Decrease of the Amount of Fluid in the Blood. In intimate connection with the disturbance of the nerves and circulatory functions just described is the decrease in the amount of fluid in the blood. This is due to increased secretion in the intestine with absence of absorption, to romiting, and to increased perspiration. As a consecpuence there exist dryness of the tongue and a tormenting thirst; the urine is also passed only in small quantities, and in some instances there may eren be anuria.

Certain symptoms which occur but rarely and also belong more or less to this group are cramps, tetanus, coma, delirium, fever. Whether these symptoms are due to auto-intoxication or to other factors (especially the dry condition of the blood) is as yet not settled.

cujuciuie Nigns. - Inspection reveals eitnes: a symmetrical fulness of the abdomen (sometimes barrel shaped) or a protrusion of eertain parts. Thus, as mentioned above, the upper part of the abdomen is protruded when the occlusion involves the duodenum or the upper part of the jejunum. The right iliac region is intensely tympanitic if the occlusion involves the hepatic flexure, while the left iliac region is the seat of the, protrusion if the occlusion involves some portion of the descending colon. After the disease has existed for some days there is as a rule a general marked swelling of the abdomen.

Palpation reveals in some cases a circumscribed area which is painful on pressure and thus serves to localize the seat of the disease. This is especially the case very soon after the onset of the symptoms. In the larger number of cases, however, there is a special tendervess either in the region of the navel alone or over the entire abdomen. In comparatively few cases will palpation reveal a tumor situated deeply within the abdomen and in direct connec- 
tion with the site of obstruction. This occurs especially in intussusception, strangulation, in occlusions due to compression by tumors, and in fecal impaction. After a thorough palpation of the abdomen a digital examination of the rectum and also of the vagina should be performed. It is hardly necessary to add that a thorough examination should be made of any existing hernia which may be the seat of incarceration.

By means of auscultation either from a distance or in the immediate neighborhood of the abdomen we are often enabled to judge about the state of the intestinal peristalsis; for when the latter takes place in a violent mauner splashing and gurgling noises are always audible.

Percussion is usually of great importance. In general meteorism it permits us to judge of the position of the diaphragm and liver. If percussion shows a change in character over a certain region of the abdomen during a period of a few minutes, it follows that the condition of an intestinal coil lying beneath has undergone some change in its state of fulness, and thus indicates that the bowel is still in active peristalsis. Auscultation and percussion may be used conjointly and serve the same purpose. In case no change whatever is noted on percussion for a very long period of time, there is a suspicion that paralysis of the bowels exists. The liver dulness will be found either partly or entirely absent in almost all cases of perforation, but in some rare instances even without perforation. In the latter event we must assume that intestinal coils filled with gas are lying above the liver. I have observed such a case with recovery during the last year. Sometimes percussion may help to discorer existing exudation, dulness being found in the lower part of the abdomen.

Examination of the romited matter will show the pres- 
ence or absence of fecal elements. The urine is scanty, very concentrated, often contains albumin, and almost always shows an increase of indican and phenol. Rosenbach's reaction is almost always present.

Course. - The course of an acute obstruction will depend first upon its location, and secondly upon its nature. The higher up in the intestine the obstruction is situated the more rapid as a rule is the course of the disease. Volvalus and strangulation of the intestine are generally accompanied by a more violent course than is obturation by foreign bodies. The duration of the disease is not always the same. In some instances the patient dies very soon, a few hours or a day or two after the commencement of the obstruction, of shock and paralysis of the heart. In other instances the disease lasts sereral days or even a week. In intussusception the duration of the disease is longer, several weeks, showing periods of exacerbations and remissions.

If the patient recovers from the collapse and there is a spontaneous re-establishment of the patency of the intestinal lumen (i.e., the obstruction is relieved, which may happen in cases of invagination, torsion, and obturation by foreign bodies), there is at first as a rule a passage of flatus, which may be followed by a fecal movement of offensive odor. In case of invagination there is often some blood in the evacuation. All the symptoms which have previously existed begin to abate, the fecal vomiting ceases, the meteorism becomes less, and the patient gradually recovers from his severe illness. In cases in which the intestinal obstruction has led to considerable anatomical changes within the lumen of the bowel (úlcers, gangrenous processes, adhesions), after a period of comparatire enphoria, symptoms of chronic intestinal obstruction may develop. 
In the greater number of cases of acute intestinal obstruction the latter persists, and the patient, if not operated upon, generally dies of diffuse peritonitis, with or without perforation of the intestines. Even without perforation, peritonitis may readily develop in consequence of the paralytic state of the intestine; for, according to Bünnecken, ' bacteria can easily penetrate the intestinal wall as soon as the latter is in a paralyzed condition and thus give rise to inflammation of the peritoneum.

Circumscribed peritonitis around the occluded part need not give distinct symptoms. General peritonitis, however, always enhances the alarming symptoms already existing. Thus the meteorism increases; the dyspnoea, hiccough, and vomiting become more violent, the pains unendurable; the heart begins to give ont and pronounced collapse appears. Generally there is a rise of temperature and frequently a fluid exudation within the abdomen is discoverable. If perforation of the intestine has taken place, the symptoms just described appear still earlier and with more violence. The abdomen becomes more or less rounded and the diaphragm is pushed upward in the highest degree. The liver dulness disappears and the pains become excruciating. The shock may be so great that the patient becomes unconscious and remains so until death brings relief.

Complications appearing during the disease may also be the cause of death. Thus deglutition pueumonia (Schluckpneumonie) which occasionally occurs by aspiration into the lungs of gastric and intestinal contents during the act of vomiting, or septicæmia in consequence of intestinal perforation, may develop with embolic processes in the lungs, liver, and other organs. In exceptional cases there occurs

'Bönnecken : Virchow's Archiv, Bd. 120. 
an adhesion of the occlnded intestinal coils to the anterior abdominal wall, and after the gangrenous destruction of the latter as well as of parts of the gut, an anus praternaturalis develops, or a fistulous opening between two portions of the intestines, or again a fistula of the intestine into the bladder, uterus, vagina, or stomach.

Diagnosis. - The diagnosis must deal with the following three points: A. Recognition of the intestiual obstruction. B. Its seat. C. Its etiological factor.

A. Recognition of the Intestinal Obstruction. The recognition of an acute intestinal obstruction is not difficult if the symptoris described above are present in a marked degree. Thus total absence of passage of fecal matter and flatus combined with symptoms of collapse, meteorism, pains, and fecal romiting will permit a positive diagnosis of intestinal obstruction. In many instauces, however, only a few of the symptoms mentioned are present, and then the diagnosis is quite difficult. The symptom of the greatest diagnostic value is fecal vomiting, although even this alone does not always warrant the diagnosis of obstruction, for it also occurs in intestinal paralysis. The latter condition must be especially borne in mind in cases in which there has been a history either of contusion of the abdomen or of a reposition of incarcerated hernia shortly before the appearance of the disease. The fecal romiting of hysterics can also be easily recognized, as there are always symptoms present which indicate the true condition.

The greatest difficulty in diaguosis lies in the differentiation between intestinal obstruction and diffuse peritonitis, especially if the latter accompanies appendicitis. All the symptoms characteristic of intestinal obstruction may occur also in peritonitis. A thorough consideration of all 
the symptoms and their differentiation in these two diseases will, however, permit a decision.

The following points will serve as a guide in this connection: In acute peritonitis there is a rise of temperature at the beginning of the disease, while in intestinal obstruction there is at first no fever or even a subnormal temperature. There are exceptions, however, and a general peritonitis of a grave nature may run its course without any fever but with symptoms of collapse. The pains on pressure over the abdomen are much more intense in peritonitis; in intestinal occlusion the spontaneous pain may occasionally even be relieved by pressure. Fecal vomiting is of comparatively rare occurrence in peritonitis, and if present it usually appears later than in intestinal obstruction. The meteorism is diffuse in peritonitis right from the start: It thus causes a general distention of the abdominal parietes. In obstruction the accumulation of gas is at first less pronounced, circumscribed, and increases gradually. In peritonitis the abdomen becomes tense from the first, while in obstruction, at the commencement at least, it is as a rule soft. The existence of an exudation speaks in favor of general peritonitis. In peritonitis accompanying appendicitis there will be besides the above symptoms the phenomena characteristic of the latter disease. In some instances, however, the differentiation between peritonitis and obstruction will hardly be possible and mistakes are liable to occur.

Acute intestinal obstruction is occasionally simulated by poisoning with arsenic and also by a very severe attack of cholera. In the former condition there will be a history of poisoning, and in the latter the presence of cholera bacilli in the dejecta will clear up the diagnosis. In rare instances a severe attack of biliary colic or of renal colic 
may in some respects resemble intestinal obstruction. A thorough examination, howerer, will always reveal the true condition. In biliary colic as a rule there is swelling of the liver and sometimes jaundice; in reual colic the pains radiate from the kidney to the bladder, there is a burning sensation during urination, and the urine often contains mucus and occasionally a few pus corpuscles or blood cells. Intestinal colic resulting from chronic lead poisoning occasionally simulates true obstruction of the bowels. The anamnesis, howerer, will show that we have to deal with lead poisoning. Besides, in these cases there is, as a rule, a more or less sunken condition of the abdomen. Simple intestinal colic (of nerrous origin) will hardly erer give rise to mistakes in the diagnosis, as the clinical picture is less severe and the disease quickly subsides.

B. Location of the Obstruction. The location of the seat of the obstruction is not merely of theoretical value, but of great practical importance, for this decides the question as to where abdominal incision should be made in cases of operation. It will be useful to discuss first at what point of the abdomen the obstruction is situated, and secondly, what particular portion of the bowel it involves.

1. The point at which the patient first experiences pain is significant in case he is able to locate it definitely. In many instances, however, the pain is not experienced in one circumscribed spot, and is often located diffusely in the neighborhood of the navel. The presence of a tensely tympanitic intestinal coil, which does not change its configuration and thus makes the abdominal wall protrude asymmetrically, is of great importance; for, according to Von Wahl, such a coil is often found above the occluded segment of intestine. Strong peristaltic contractions ruuning in the same direction over a certain region of the ab- 
domen, especially if they return periodically and always in the same area, will serve to locate the place at which the obstruction is situated. For these peristaltic waves pass along the intestine down to the seat of the obstruction, which they are unable to overcome.

Palpation of the abdomen occasionally reveals the presence of a sausage-like tumor. This occurs especially in cases of intussusception. If such a tumor is present, the location of the obstruction is certainly easy. A thorough examination of all hernial openings will occasionally rereal an incarceration of the intestine and also show the site of the lesion. If there is no hernia the examination must be continued through the vagina and through the rectum. The exploration through the vagina will show whether the pelvic organs are normal, and if not, whether a tumor oringting from the genital organe is csiapressing the intestines. Digital examination of the rectum will enable us to discover a stricture, an intussusception, or a tumor of the lower portion of the bowel. In some cases a thorough examination of the entire rectum and the descending colon may be undertaken with the whole hand under chloroform narcosis, according to the method of Simon. In cases of intussusception involving the sigmoid flexure and rectum, the anus often remains open (paralysis of the sphincters) and there appears an involuntary evacuation of a mucobloody fluid from time to time.

2. Determination of the Portion of the Intestinal Tract in which the Obstruction is Situated. Small Intestine. If the obstruction is situated in the small intestine all the symptoms (pains, vomiting, collapse) are, as a rule, much more intense and appear sooner than in obstruction of the large bowel. Soon after the commencement of the disease, there is copious vomiting which may become fecal after a short 
period. The meteorism at the beginning is localized in the upper part of the abdomen, while the lower part remains unchanged. Pronounced visible peristaltic wares in the small intestine also point to an occlusion situated within the latter.

Jaffé ' was the first to show that obstruction of the small intestine gives rise to pronounced indicanuria. As early as the second or third day of the obstruction, indican can be found in the urine in large quantities. In obstruction of the large bowel there is as a rule no indicanuria, and if it appears it does so only later in the disease, on the sixth or serenth day. The higher up in the intestinal tract the obstruction is situated, the sooner and the more frequently anuria may appear. Injections of water into the bowel may secure a fecal evacuation. The colon can also be filled with a large amount of water or gas.

If the obstruction is sitnated within the duodenum or in the upper part of the jejunum, it can often be easily recognized. Obstruction of the duodenum above Vater's papilla will manifest the same symptoms as acute dilatation of the stomach in consequence of a stricture. There will be ischochymia and continuous romiting of chyme. An obstruction situated within the duodenum below Vater's papilla will give rise to romiting of large quantities of pure bile. The vomited matter may contain acids from admixture of gastric juice. It is never fecal in character. The gastric region is protuberant but sinks in after a spell of vomiting.

If the obstruction is situated within the beginning of the jejunum the vomiting assumes at first a greenish hue (decomposed bile) which may be followed by the romiting of pure unchanged yellow bile. Occasionally the romited matter assumes a fecal character. Obstructions situated

1 Jaffé : Centralbl. f. die med. Wissenschaften, 1872. 
within the duodenum or at the beginning of the jejunum, as a rule, are unaccompanied with indicanuria.

Obstruction of the Larye Boucel. The symptoms here are nsually less violent and appear a little later than in the obstruction of the small intestine. Fecal vomiting often appears long after the establishment of the occlusion, and it may even be absent if the obstacle is situated at the begiuning of the descending colon or lower down. The meteorism is in most instances limited to the lower parts of the abdomen and also to the lumbar regions. In occlusion of the descending colon it may be noticeable that at first there is a protrusion in the left iliac region, afterward a protrusion of the transverse colon, and ultimately the ascending colon will also become tympanitic. As mentioned above, indicanuria will be absent during the first five or six dars of illness.

With regard to the determination of the occlusion within the lower parts of the colon, Brinton's ${ }^{1}$ method, already in use over fifty years ago, is very valuable. It consists in filling up the bowel with water through the rectum. If not more than half a quart can be injected, the obstruction must be situated in the upper part of the rectum. If one to two quarts can be injected, the obstruction must be situated above the sigmoid flexure, in the descending colon, or still higher. In case obstruction is situated in the ascending colon four quarts or still more can be injected and retained in the bowel. Insufflation of air or carbonic acid gas into the rectum will also occasionally show the seat of the obstruction, if the latter is situated in the descending or the transverse colon, as there will be a filling up with gas of the free portion of the bowel up to the obstructed point. When the obstruction is located beyond the trans'Brinton: "On Intestinal Obstruction, " London, 1867. 
verse colon, however, it will not permit of distinct recognition by this method.

C. Recognition of the Different Forms of Acute Obstruction. If the diagnosis of acute obstruction of the bowels is not always easy, the recognition of the special anatomical lesion underlying it is still more difficult. In many instances an exact anatomical diagnosis will not be possible and we will have to be satisfied with a probable conjecture. In some cases, however, the exact determination of the etiological factor underlying the obstruction will be possible. The following groups of acute obstruction of the bowels can be clinically differentiated :

1. Acute Incarceration of the bowels in hernias (also internal hernias, in slits of the omentum, mesentery, or diverticula), in strangulation by bands or twists of the bowel, is most frequent between the ages of twenty and forty. It occurs more often in males than in females. There is often a previous history of peritonitis, of hernia, or of accidents (contusions). The onset of the disease is sudden. The pains are severe. Vomiting is present from the start, becoming stercoraceous later on. Collapse is marked. Tenesmus is absent. Physical examination of the abdomen gives, as a rule, negative results.

2. Volvulus most often involves the sigmoid flexure and can then be easily recognized. Volvulus of the small intestine, which occurs very rarely, camnot be differentiated clinically from incarceration. The rotation of the bowel around its axis is either complete $\left(360^{\circ}\right)$ or incomplete (half rotation, $180^{\circ}$ ). In the first instance there is total occlusion, while in the latter the intestinal lumen is at first partially pervious. Volvulus is more common in males than in females in the proportion of four to one, and occurs principally late in life, usually between forty and sixty. 
There is usually an antecedent history of chronic constipation. The onset of the disease is sudden. The pain ordinarily is intermittent. Vomiting may be absent at first and later on occurs intermittently. Constipation is almost absolute and grows worse after the use of aperients. There is pronounced meteorism. The sigmoid flexure can occasionally be felt as a tumor. Only moderate amounts of water can be injected into the rectum.

3. Intussusception occurs very frequently in early childhood. The ouset is sudden, the pains appear early, are colicky in character and come in paroxysms. There are marked tenesmus and bloody evacuations. The collapse is not pronounced. The invaginated coil may be accessible to palpation and then appears in the form of a tumor of egg-size or somewhat larger, this occurring in about fifty per cent of the cases. Meteorism develops in conjunction with peritonitis.

4. Obturation of the Intestine by Gall Stones, Enteroliths, or Foreign Bodies. Obstruction by gall stones occurs chiefly in women and is more frequent at an advanced age. A previous history of gall stones or a preceding attack of jaundice, pains in the region of the liver, and swelling of this organ are points which aid in the diagnosis. Obstruction by gall stones usually occurs in the small intestine; the symptoms, as a rule, are less severe than in other forms of ileus. The collapse is not pronounced or may be entirely absent. Flatus may occasionally be passed, copious vomiting of bile may be present. If the gall stone is situated in the lower portion of the ileum the vomiting may later become stercoraceous. Occasionally the stones can be palpated through the abdomen and felt as a hard mass. Meteorism is generally not highly developed. In some instances there is diarrhœe with admixture of blood, the 
latter being due to abrasions of the intestinal mucosa produced by friction of rough gall stones.

The recoguition of an enterolith as the cause of obstruction is very difficult and possible only when small fragments of a fecal calculus have previonsly been found in the dejecta. The seat of obstruction is as a rule then in the large bowel, the latter being the place where enteroliths develop.

Obstruction by foreign bodies will be recognized by the previous history; often also, especially if they are of a metallic nature, by a Roentgen picture. An accumulation of cherry pits or plum stones may also cause an obstruction and will likewise be recognized by the previous history and by the presence of some of them in the dejecta.

Hardened fecal matter will very rarely give the picture of obstruction. This will occur only in very weakened individuals and in persons with spinal trouble. In these cases the rectum and colon will be found filled with greatly hardened scybala. If a stricture or a tumor exists within the intestine and narrows its lumen, an accumulation of fecal matter above the stricture gives rise to acute obstruction.

5. Dynamic Ileus. Obstruction due to paralysis of a segment of the bowel can be recognized only with great difficulty. Often there has been a preceding laparotomy or some operation on the genital organs in the female or a history of a replaced hernia.

With regard to the recognition of the different forms of intestinal obstruction the following table, which gives the frequency of the principal symptoms in the various forms of obstruction, may be of assistance.

Among two hundred and ninety-five cases of acute ob17 
struction of the bowels collected in literature and minutely examined by R. Fitz ${ }^{1}$ of Boston, the symptoms were as follows with regard to the different groups of obstruction:

\begin{tabular}{|c|c|c|c|c|c|}
\hline & $\begin{array}{l}\text { Strangu- } \\
\text { lation, } \\
\text { Per Cent. }\end{array}$ & $\begin{array}{l}\text { Intussus- } \\
\text { ception. } \\
\text { Per Cent. }\end{array}$ & $\begin{array}{l}\text { Twist. } \\
\text { Per Cent. }\end{array}$ & $\begin{array}{c}\text { Gall } \\
\text { Stones. } \\
\text { Per Cent. }\end{array}$ & $\begin{array}{l}\text { Stricture } \\
\text { or Tumor. } \\
\text { Per Cent. }\end{array}$ \\
\hline Pain ............. & 82 & 70 & 60 & 83 & 60 \\
\hline Nausea and vomiting.... & 69 & 75 & 37 & 74 & 80 \\
\hline Fecal vomiting ......... & 47 & 13 & 15 & 61 & 33 \\
\hline Tympanites .......... & 56 & 33 & 55 & 56 & 66 \\
\hline l'umor................ & 10 & 69 & & 13 & 27 \\
\hline Visible coils .......... & 11 & .. & 7 & .. & 20 \\
\hline
\end{tabular}

Prognosis. - The prognosis of acute obstruction of the bowel is very serious. According to Curschmann, ${ }^{2}$ only thirty to thirty-five patients out of one hundred recover from this disease. As a rule ileus caused by coprostasis or by obturation with gall stones and foreign bodies gires the best prognosis. Then come volvulus and intussusception, while incarceration gives the worst prognosis. If in the course of ileus deglutition pneumonia or diffuse perjtonitis or perforation of the bowel derelops, then the case is well-nigh hopeless. Operative interrention, especially in cases in which the seat of the intestinal occlusion is known, improves the prognosis considerably, but only if it is resorted to early. Later, when the complications just mentioned arise, not much can be expected from an operation.

\section{Treatment.}

A. Medical Treatment.-Absolute rest is of the greatest importance. The patient should be kept in bed and told to avoid any abrupt motions. He should not be

'R. Fitz: "Transactions of the Congress of Physicians and Surgeons," vol. i., 1888.

'Curschmann: "Die Behandlung des Ileus." Congress für innere Medicin, Wiesbaden, 1889. 
allowed to go to the water-closet, but should use a bedpan. With Treves, Graser,' ${ }^{1}$ and others I am for absolute rest of the stomach and intestines, i.e., no food whatever should be given to patients suffering from acute obstruction of the bowels.

If there is great thirst a teaspoonful of hot water or very weak tea may be given every half-hour or hour or a small piece of ice may be held in the mouth until it melts, but the water should not be swallowed. Neither should any stimulants like wine, champague, or whiskey be given by the mouth. In obstruction of the small intestine small quantities of a saline solution (about seven to twelve ounces) may be injected into the bowel several times during the day. If the sickness lasts several days, nutritive enemas consisting of milk and egg or of a peptone solution may be given in the same way. If, however, the patient is not able to retain the enema, consilerable quantities of saline solution must be injected either subcutaneously or intravenously.

All writers agree that no cathartic remedies whatever should be used, as they increase the peristalsis and thereby may cause great harm. A cathartic should be permitted only in cases in which the obstruction is positively due either to gall stpnes or hardened fecal masses or in dynamic ileus. It is, however, of benefit to evacuate the lower parts of the bowel by means of an enema. This cleans out the rectum, diminishes the feeling of tension to a slight extent, and prepares the bowel for the nutrient enemas.

The administration of opium plays a principal part. It

1 Graser: "Behandlung der Darmverengerung und des Darmrerschlusses." Penzoldt-Stintzing's "Handbuch der speciellen Therapie innerer Krankheiten," Jena, 1896. 
is indicated not only as a means of allaying pain but for its soothing action upon the intestinal peristalsis. The arrest of the latter may have a direct curative effect, since it may promote a return of the partly incarcerated or invaginated or slightly twisted coil to its normal position.

In order to secure a prompt action of the drug it is best to first give a hypodermic injection of morphine, one-sixth to one-fourth of a grain. A short time afterward a suppository of two-thirds of a grain of opium is administered and repeated every three or four hours until the pains are kept in abeyauce. In cases in which the romiting is not so marked, opium may be given in the form of the tincture fifteen to twenty drops every three to four hours. It is harelly necessary to say that the opiates should not be used too lavishly. Only so much should be administered as is absolutely necessary for relieving the pain and quieting the violent peristalsis of the intestine. Given in this way, opium not only acts as a sedative but also as a stimulant on the heart. Patients in deep collapse very soon after an injection of morphine become warm, show a better pulse and a more normal temperature. The only disadvantage of opium is that it slightly masks the true picture of the disease. It is therefore best whenever possible first to make an exact diagnosis by thorough examination of the abdominal viscera by palpation, auscultation, etc., before administering it.

If the symptoms. of the disease persist after the administration of opium, especially if the tension of the abdomen is not relieved and no flatus is passed, it is well to discontinue the remedy for a certain period of time. This will enable the physician to judge the situation critically.

As a further sedative agent applications of poultices can be considered. A hot-water bag, a hot plate wrapped up 
in flannel, or a Japanese warm box, wet packs (Priessnitz) are useful. If there are signs of peritoneal inflammation, applications of ice or of very cold poultices are preferable.

Lavage of the stomach was first recommended in this disease by Kussmaul and Cahn.' This procedure is of benefit if the obstruction is situated high up in the small intestine. It empties the stomach, relieres the romiting, and also decreases the abdominal tension. There is no doubt that this therapeutic measure is sometimes crowned with success in appropriate cases. As a striking instance of the efficacy of this mode of treatment the following case may be reported:

E. K., thirty-fire years old, had always been well, when he suddenly became critically ill with violent abdominal pains and constant vomiting. For three days there was no evacuation of the bowels nor was the patient able to pass any flatus. On examination I found his abdomen considerably distended and tense. The stomach could be mapped out and was considerably dilated, the greater curvature extending a hand's width below the navel. On palpation there was considerable tenderuess all over the abdomen. The pulse was quite frequent (110) and weak, temperature $96.5^{\circ}$ in the mouth, the extremities were cold. The face showed an expression of great suffering. There were almost continuous hiccongh and now and then vomiting of a watery, turbid, somewhat brownish-looking liquid with fecal odor. On introducing the tube over a quart of liquid of the same character was obtained. The stomach was then washed out with several quarts of water until the fluid returned quite clear. The patient felt somewhat relieved. The vomiting stopped and on the following day there was a spontaneous evacuation of the bowels. The patient was now able to pass flatus, the distention sub-

'Kussmaul-Cahn: "Heilung von lleus durch Magenausspülung." Berl. klin. Wochenschr., 1884, Nos. 42 and 43. 
sided, and he gradually recuperated. For the sake of completeness I would add that besides washing out the stomach, the treatment consisted in the administration of opium suppositories.

Lavage of the Bowel. Injections of large amounts of water into the bowel under considerable pressure are also occasionally of benefit, especially in cases of intussusception of the colon or when a foreign body or hardened fecal matter is the cause of the obstruction within the large bowel. According to Treves, it is desirable to use this procedure after anæsthetizing the patient. A considerable quantity of water (varying according to the age of the patient from half a pint to three quarts) is introduced into the bowel by means of an ordinary fountain syringe. The fluid is allowerl to remain in the colon for at least ten minutes. While injecting the water it is best to have the patient in such a position that his head is lowered and his pelvis is raised. While the irrigation of the bowels is going on the physician should hold his hand upon the patient's abdomen and in this way notice any change which may occur.

In intussusception when the tumor can be felt the latter will in some instances suddenly disappear, giving way to the pressure of the water. Too great force, however, should never be used, as this may bring on rupture of the bowels. Instead of water, injections of warm olive oil, which were first recommended by Kussmaul and Fleiner, may be used in the same way. Dr. Klubbe' has related three cases of cure by means of this method.

Inflation of the Bowel with Air or Certain Gases in. Cases of Invagination. Trastour ${ }^{2}$ recommended inflation

${ }^{1}$ Klubbe: British Medical Journal, November 6th, 1897.

2 Trastour: Bulletin Général de Thérapie, 1874, p. 107. 
of the bowel with air by means of a common bellows, to which an India-rubber nozzle and rectal tube had been attached. The forcible filling up of the bowel with air is capable of producing the same effect as the injection of water and may free the invaginated portion. Von Ziemssen ' has recommended the use of carbonic-acid gas, while Senn ${ }^{2}$ suggested hydrogen gas. Carbonic-acid gas is best used in the form of "sparklets," as suggested by Dr. A. Rose ${ }^{3}$ of New York. Care must be taken not to fill up the bowel too quickly and too forcibly.

Massage. Massage has been recommended by several writers. Its use, however, is not entirely harmless. It can be of benefit only in cases of obstruction by gall stones and fecal matter, but even in these cases extreme care in its use is necessary.

Electricity. Electricity has especially been recommended by Boudet. Among serenty cases of ileus Boudet * had fifty-three recoveries by this method. The faradic or galvanic current may be used. In the application of the faradic current one metal electrode of cone shape is inserted into the rectum while another large plate electrode is kept orer the abdomen for about ten to twenty minutes. In using the galvanic current it is necessary to have a special rectal electrode, which is constructed in such a way that water running through it forms the conductor, so as to avoid burning the mucosa. The other electrode is placed over the abdomen. The negative pole should be inside. The strength of the current should rary from ten to fifteen milliamperes. The duration of the treatment should be twenty to twenty-five minutes.

1 Von Ziemssen : Archiv für klinische Medizin. Bd. 33. Heft 3 and 4.

2 Nic. Senn : "Intestinal Surgery," Chicago, 1889, p. 244.

${ }^{3}$ A. Rose : New York Med. Journal, 1900, i., p. 47.

-Boudet: Progrìs Médical, February 7 th and 14th, 1885. 
Electricity will be of special value in obstruction due to hardened fecal matter or in the paralytic form of ileus, while in incarceration it is rather contraindicated.

Puncture. Puncture of the distended bowel has recently been recommended anew by Curschmann,' von Ziemssen, aud others. According to Curschmann, puncture of the intestine is performed in the following way: A long aspirator needle of thin calibre (like that of a Pravaz syringe) provided with a stopeock is thrust into the abdomen over a prominent coil of the intestine. A piece of rubber tubing is then connected with the outer end of the needle; the free end of the latter is inserted into a bottle filled with water, which is turned upside down in a basin likewise filled with water. The stopcock of the aspirating needle is now opened and the gas escaping from the intestinal coil appears in bubbles rising to the upper part of the bottle, displacing the water. There is no doubt that considerable temporary relief can be afforded by this mode of procedure, as it lessens the feeling of tension. Occasionally it may also have a direct curative result. Thus Curschmann reports three cures by this method. Puncture, however, is not entirely free from danger. In cases in which the intestine is already partly paralyzed, the opening after the withdrawal of the needle may not entirely close and intestinal gases and contents may continue to ooze out and cause peritonitis.

Most surgeons of note are against this procedure, as it lacks precision and is not free from danger. Thus Treves,' Kocher, ${ }^{3}$ and Graser ${ }^{4}$ are all opposed to its employment.

${ }^{1}$ Curschmann Deutsche med. Wochenschrift, 1887, No. 21.

2Treves: “Intestinal Obstruction," New York, 1899, p. 471.

${ }^{3}$ Kocher: "Mittheilungen aus den Grenzgebieten der Medizin," 1898. Bd. 4, p. 2.

GGraser: Penzoldt-Stinzing's "Handbuch," Bd. 4, p. 562. 
Sad experiences with puncture have been reported by Frentzel, ${ }^{1}$ Fürbringer, ${ }^{2}$ Hoffmann, Körte, ${ }^{3}$ and Graser. The latter observed the appearance of fecal matter and consecutive peritouitis from such an opening. $\mathrm{He}_{\Theta}$ considers puncture permissible only if the patient absolutely refuses an operation.

Mercury (Mercurius Vivus). The internal administration of pure mercury in tablespoonful doses was highly esteemed as a remedy for ileus by the old physicians. When all resources had been exhausted withont success, mercury was given as an ultimum refugium. Even nowadays many physicians are convinced of its efficacy. The use of mercury in incarceration, strangulation of the bowel by twists or bands, intussusception, is not permissible, as it does real harm. In ileus in consequence of coprostasis or in dynamic ileus, mercury may be employed if all other remedies have proven futile. Its effect consists in the penetration of the mercury into the accumulated fecal matter, thus softening it.

All the enumerated internal methods of treatment must be applied, first, in cases in which the obstruction is due either to gall stones or to foreign bodies or fecal accumulation or volvulus of the sigmoid flexure; secondly, in cases in which the exact diagnosis as to the kind of obstruction is not settled, and which are not of a very severe type. In all other varieties of intestinal obstruction and eren in the types just mentioned, after the failure of the medicinal measures at hand, an operation should be resorted to.

${ }^{1}$ Frentzel: Deutsche Zeitschr. f. Chirurgie, Bd. 33.

'Fürbringer : Verhandl. des 8ten Congresses f. innere Medicin, 1889.

: Körte : Ibidem. 


\section{B. Surgical Treatment.}

Treves, the greatest authority on intestinal obstruction, says: "There is one measure for the treatment of acute intestinal obstruction, and that is by means of laparotomy. The operation should be performed at the earliest possible moment, as soon indeed as the diagnosis is reasonably clear. In case of acute abdominal trouble in which the diagnosis" is not clear, the better and safer course is to operate." This riew is now generally accepted by physicians as well as surgeons.

As mentioned above, obturation, ileus, and volvulus of the sigmoid flexure are the only groups of intestinal obstruction in which medical treatment plays a prominent part. The importance of an early operation has been shown by Naunyn, who found that among two hundred and eighty-eight cases of ileus operated upon, the results were the more favorable the earlier recourse was had to surgical intervention. In those cases in which the operation was performed during the first two days of sickness recovery took place in seventy-five per cent. During the third day and still later there were only thirty-five to forty per cent of recoreries.

A similar view is expressed by Gibson, ' who dealt particularly with acute intussusception. Among one hundred and forty-nine cases of this affection he found an average mortality of fifty-three per cent. The first and second days showed mortality inferior to the general mortality, while the four succeeding days showed a steadily increasing mortality, in each instance greater than the average. With regard to treatment by inflation of the bowels by enemata

${ }^{1}$ C. L. Gibson: "Mortality and Treatment of Acute Intussusception, with Table of 239 Cases." Medical Record, July 17th, 1897. 
of fluids (or air) Gibson says: "It is probably not an exaggeration to say that if all cases of intussusception were treated on the onset, or say within forty-eight hours, by abdominal section, without any previous attempt at reduction, the mortality, while still cousiderable, would in all probability be rery much less than the present figures." Gibson believes that injections should be tried only on the first or at the latest on the second day.

In the discussion on intestinal obstruction which took place at the New York State Medical Association,' all the speakers (Parker Syms, E. D. Ferguson, George D. Stewart, J. W. Gouley, J. D. Rushmore, LeRoy J. Brooks, John F. Erdmann, Fred. H. Wiggin, and H. O. Marcy) were in favor of surgical treatment and for early interrention. J. D. Rushmore says:" "I have no hesitation in affirming that in competent hands operation for intestinal obstruction would not have a mortality above twenty per cent. In my personal experience, including orer one hundred and ten operations, the mortality has been nearly forty per cent. In the last thirty cases there have been six deaths." Wiggin ${ }^{3}$ considers that operations performed within the first forty-eight hours will give a mortality of 22.2 per cent.

Inasmuch as the question of operation has to be dealt with in each case of intestinal obstruction, it is adrisable to have the opinion and advice of an expert surgeon right at the start of the disease. The physician and surgeon should act together, the first watching the symptoms carefully and making the diagnosis, the second prepared to resort to surgical interrention as soon as it is demanded.

1 Transactions of the New York State Medical Association, 1898.

2 J. D. Rushmore : Ibidem.

3F H. Wiggin : Da Costa's "Modern Surgery," p. 644. 
In this way the number of recoveries will be greater in the future than it has been in the past.

The operation consists in making an abdominal incision, finding the seat of the lesion, and removing the obstacle if possible. If not, an enterostomy is performed in the most distended coil of intestine which is then attached to the abdominal wall. The fecal matter and the gases thus find an outlet through this opening. Enterostomy is also required in all cases in which the portions of the intestine are already found gangrenous. Treves says that this operation (enterostomy) could be avoided in acute intestinal obstruction if the abdomen were opened at the very earliest possible moment. Every hour delayed adds to the grarity of the case. "The earlier the operation the less the need for enterostomy. Laparotomy should be performed at an early enough period to render an opening into the bowel unnecessary."

\section{CHRONIC INTESTINAL OBSTRUCTION.}

Etiology.-Chronic intestinal obstruction may be caused by the same factors which produce acute ileus if they do not occlude the entire lumen of the bowel but leave part of the canal open. Besides, obstruction of the intestine is frequently occasioned by strictures resulting from preceding ulcers or from new growths. The latter, benign as well as malignant, are liable to give rise to occlusion even if they do not occupy the entire circumference of the bowel, by simply obtruding part of the canal at the site of their greatest development. Strictures caused by ulcers much more frequently involve the large than the small intestine. According to Treves, they are found six times as often in the large bowel as in the small one.

While formerly dysentery was believed to be the cause of a 
large number of these intestinal strictures, Woodward ' has shown that this view is not supported by facts. Among the many autopsies on patients with chronic dysentery which the latter had an opportunity to observe, there was not one case of dysenteric stricture of the intestine. Nothnagel agrees with Woodward. On the other hand, tuberculous ulcers of the intestine which were regarded as ouly rare causes of intestinal stricture have recently been found to produce strictures quite frequently. Koenig ${ }^{2}$ laid stress upon the frequency of constricting tuberculosis of the intestines. The latter may exist even if tuberculosis in other organs is absent. 'Ulcers of typhoid fever very rarely if ever cause strictures, and this also applies to the small follicular ulcers. Syplilitic ulcers on the contrary produce strictures quite often. All kinds of strictures are met with most frequently in the lower portion of the colon, principally in the rectum. Sometimes they lie just above the anal region and can then be very easily discovered.

Symptomatology. - The symptoms and the course of the disease vary considerably, and greatly depend upon the cause of the obstruction. Thus, clinically, the benign growths must be differentiated from the malignant ones (in which the obstruction is caused by cancer). The picture which the intestinal obstruction as such produces will, however, be pretty much the same. A stenosis which is not very much pronounced may give rise to no symptoms whatever. It is therefore quite evident that the disease may exist for some length of time before manifesting its presence.

In typical cases of chronic intestinal obstruction the onset is slow and insidious. The patient at first notices

1 Woodward: Loc. cit.

${ }^{2}$ Koenig : Deutsche Zeitschrift für Chirurgie, 1891. 
slight digestive disturbances, some discomfort in the abdomen which gradually chauges into real pain, and slight constipation. The latter as a rule quickly becomes worse. Mild aperients which a short while before were efticient refuse to act, and the patient is obliged to resort to stronger cathartics; at times even these will fail to work. Frequently constipation suddenly alternates with an attack of severe diarrhœa, which may last several days and be followed by another period of obstivate constipation. In some instances the color and form of the fecal matter will be an indication of the seat of the stenosis. It is generally believed that pipestem-like or tape-like motions indicate a stricture in the colon. According to Treves, 'however, this sign is of very little value, as in the great majority of cases the sphincter muscle is the originator of these peculiar shapes. Diarrhœa may also occasionally occur. It is sometimes quite obstinate, especially if the stenosis is situated in the large bowel. An admixture of blood or pus in the dejecta is occasionally met with and is due to ulcerative processes taking place at the seat of the stricture or immediately above it.

Vomiting is not a very marked feature at first, but later on occurs more frequently. When the obstruction, however, becomes complete, romiting is a prominent symptom and may assume a stercoraceous character.

The situation of the obstruction has much influence upon the clinical picture of the disease. If the stenosis is situated in the duodenum above Vater's papilla, the symptoms will resemble those of stricture of the pylorus. Ischochymia, vomiting, nausen will be the prominent features. A stenosis of the duodenum below Vater's papilla, although presenting symptoms similar to those of stricture of the

'Treves: Loc. cit., p. 395. 
pylorus, will be recoguized by the more or less constant presence of large amounts of bile in the stomach. The farther down in the small intestine the obstruction is situated, the less pronounced are the gastric symptoms and the more marked the intestinal manifestations (less vomiting or nausea, more constipation, colicky pains). If the stenosis is situated in the lower portion of the ileum or in the colon no gastric symptoms are as a rule present. The appetite is good, there is no nansea, and the principal features are obstinate constipation, sometimes alteruatiug with diarrhoea and frequent attacks of colicky pains.

Condition of the Abclomen. The abdomen may present a normal appearance when the stenosis is situated in the npper portion of the small intestine, although in some of these cases there may be a protrusion of the upper part of the abdomen. If the site of obstruction is in the lower portion of the small intestine or in the large bowel, then some distention of the abdomen is usually noticeable, especially after the disease has advanced considerably. Abore the obstruction there is always distention and hypertrophy of the bowel. The latter is a manifestation of the attempt which nature makes in order to overcome the difficulty. The intestines above the stenosis act with greater force in order to propel the contents through the narrow passage.

The contraction of the bowel above the affected area often assumes a tetanic type and is then painful. Such violent tetanic contractions are often visible through the abdominal wall, and by propelling large amounts of liquids and gases through the narrowed lumen, give rise to gurgling and bubbling sounds audible at a distance. Treves thus describes the picture which this violent peristalsis manifests: "The surface of the abdomen becomes uneven, 
a rounded elevation appears in one place and depressions appear in another. They produce an aspect comparable to that of a relief map of a hilly country. Slowly the hill-like elevation sinks and vanishes and out of the shallow valley appear fresh eminences which rise up and move along beneath the skin. The movements are slow and attended by colicky pains, and by more or less of rumbling and gurgling sounds."... "The same coil appears again and again and can often be quite definitely recognized. Although as a rule the contracting coils of the small intestine are of considerably smaller size than those of the large bowel, occasionally even the small intestine may assume such dimensions that it cannot be differentiated from the large bowel."

Meteorism is often present. If the obstruction is situated in the lower portion of the colon or in the rectum, the meteorism is at first restricted to the large bowel, the distention then being pronounced along the course of the colon at both sides of the abdominal wall and in the epigastric region. The lower part of the abdomen and also the region of the narel may be free from meteorism. If the stricture is situated in the lower portion of the ileum or cæcum, the lumbar regions of the abdomen are quite lax, while the distention is more or less pronounced in the mesogastric and hypogastric regions.

After having described the symptoms of chronic intestinal obstruction in a general way it will be useful to point out separately the characteristics of some special forms which occur more or less frequently.

Chronic intussusception may develop either after an acute attack or begin slowly and insidiously without at first giving rise to any marked symptoms. It is most frequently found in the ileo-cæcal portion. Pain occurs during the 
progress of the disease and is usually of a paroxysmal character. Attacks of pain may appear several times a day or once in trenty-four hours. Occasionally days and even weeks elapse between the paroxysms. As a rule the intervals between the attacks grow shorter as the disease advances. In some cases there is almost continuous suffering with occasional exacerbations. Vomiting seldom occurs and is certainly not a marked feature. A tendency to diarrhoea very often exists. The bowels may be normal or constipated for a while and then become loose, or there may be persistent diarrhoea. Blood is very often passed with the stools and tenesmus is occasionally present.

On examination of the abdomen by palpation a tumor is . found in almost half of the cases. The nature of the tumor corresponds to that found in acute intussusception described above. Occasionally a tumor can be felt in the rectum when the intussusception inrolves the lower portion of the large bowel. In rare instances the invaginated portion is separated from the bowel by necrotic processes, and may then appear in the movement. While this event may in rare instances lead to perfect recovery (the other portions of the bowel growing together and the lumen thus being restored), in the greater majority it canses death through perforation, rupture of the intestinal walls, and general peritonitis.

Chronic Obstruction Due to Fecal Accumulation. This variety of intestinal obstruction is more common in females than in males and is usually met with in more advanced age and in patients suffering from hysteria and brain troubles. As a rule, the patients have already long before been subject to habitual constipation; usually many days elapse without an evacuation of the bowels. From time to time enormous quantities of fecal matter are passed 18 
by artificial means. Later on the symptoms of constipation grow more inteuse. The abdomen becomes distended and it is much more difficult to secure an evacuation even by artificial means. As a rule the patient is tormented by eructations and flatulence. His appetite is poor; he has a bad taste in his mouth and frequently his breath has an unpleasant odor. Headache, vertigo, and a general tired feeling are often encountered.

These symptoms, as well as the marked unhealthy appearance of the skin, are most probably due to intestinal auto-intoxication. Certain chromogens, the products of decomposition, are absorbed from the bowel and give rise . to this peculiar discoloration of the skin. The conjunctivæ also are often yellow. A further symptom due most probably to the same process of auto-intoxication is the rise of temperature which is often present. If the distention of the abdomen is rery marked, a feeling of oppression in the chest and palpitations of the heart are experienced.

Fecal accumulation sometimes causes pressure upon the lumbar or sacral norves and gives rise to discomfort in the genital organs or to pain in the thigh radiating down the entire leg. Distended coils may be visible through the abdomen and there may be much rumbling and gurgling heard after constipation has lasted a long period. This symptom is, however, not so marked here as in cases of stricture of the intestine. Vomiting may occur and even become stercoraceous. Slight colicky pains are felt over the abdomen, but as a rule they are not intense.

The symptoms having advanced to an extreme degree, rolief may ensue either spontaneously or after resort to different procedures which serve to evacuate the bowels. Occasionally, however, an evacuation of the bowel cannot be obtained and the patient develops all the symptoms of an 
unyielding obstruction which may be fatal. Often there are attacks of obstruction following each other at certain intervals. The narrowed lumen of the bowel most probably becomes entirely occluded or blocked by a piece of hard fecal matter, which completely fills it and cannot more in either direction. Sometimes the abrupt stoppage may be due to some bending or kinking of the distended bowel.

In almost all cases of obstruction by fecal masses a tumor can be palpated usually in some portion of the colon. The tumor is caused by the fecal accumulation. The cæcum, the hepatic and the sigmoid flexures are the places where the tumor is most often encountered. Such a fecal tumor feels hard and uneven; sometimes it has a globular shape. As a rule it is not painful on pressure. Sometimes it is possible to change the shape of the tumor by pressure. This is the best proof of its fecal character. Sometimes, however, pressure does not give rise to any change in the configuration of the mass if the fecal matter is very hard. The best sign of its fecal nature is the change in form after repeated irrigations of the bowel. In some rare instances the fecal accumulation occupies the greater part of the abdominal cavity and gives the impression of one immense tumor of very hard consistency. I have seen two such cases in patients suffering from grave melancholia. Here also after repeated irrigations of the bowel and administration of cathartics the tumor gradually becomes smaller and ultimately disappears.

Stricture of the Rectum.-In this condition as a rule there are at first merely symptoms of constipation; later on these become more obstinate, requiring strouger cathartics. The patient now begins to complain of congestion of the head, anorexia, nausea, cold feet, and sometimes of disagreeable 
sensations in his limbs; still later there is diarrhoea which may persist as such or alternate with constipation. Off and on muco-purulent material appears with the dejecta. A burning sensation is often felt in the rectum, and tenesmus is frequently present. Hemorrhoids and prolapse of the rectum often accompany the stricture.

Digital examination of the rectum often reveals a stenosed area in its lower part. The finger is either not able to pass any farther than a few centimetres (five to six) above the anus or it meets with a resistance which it can overcome. Contrary to spasm of the rectum which yields completely after the finger has succeeded in passing the constriction, in stricture of the rectum the pressure of the narrowed lumen exerted upon the finger remains constantly the same.

Most of the strictures are situated about five to six centimetres above the anus, seldom higher up. In the latter instance the examination must be made with a bougie or with a rubber tube which is not too soft. In order to determine the exact nature of the stricture it is always best to make a risual examination of the rectum by means of a speculum.

Complications.-No matter to what cause the intestinal obstruction is due, in the protracted course of the disease several complications are liable to occur, although here less often than in acute obstruction. Above the stenosed area ulcerations of the bowel may take place and perforation may occur, giving rise to general peritonitis. Occasionally circumscribed peritonitis may ensue in a similar manner and lead to an abscess surrounded by adhesions. Such an abscess may rupture through the abdominal wall and under farorable conditions (if communicating with the intestinal lumen) form a fecal fistula. In many in- 
stances the patients gradually waste away and die in consequence of thrombosis of the crural vein and decubitus.

Course and Prognosis.-The duration of chronic intestinal obstruction depends largely upon the nature of the particular affection and upon the degree of the obstruction. If there are no complications and the patients lead a perfectly rational life (with regard to diet and treatment) the condition may last a number of years. In other cases the symptoms of intestinal obstruction rapidly progress and life is then of short duration unless something radical is done.

Diagnosis. - The diagnosis of chrouic intestinal obstruction is warranted by the presence of gradually increasing symptoms of constipation, and attacks of intestinal colic with a temporary stoppage of the bowels following each other at not too great intervals. The acute attack of obstruction in these cases of

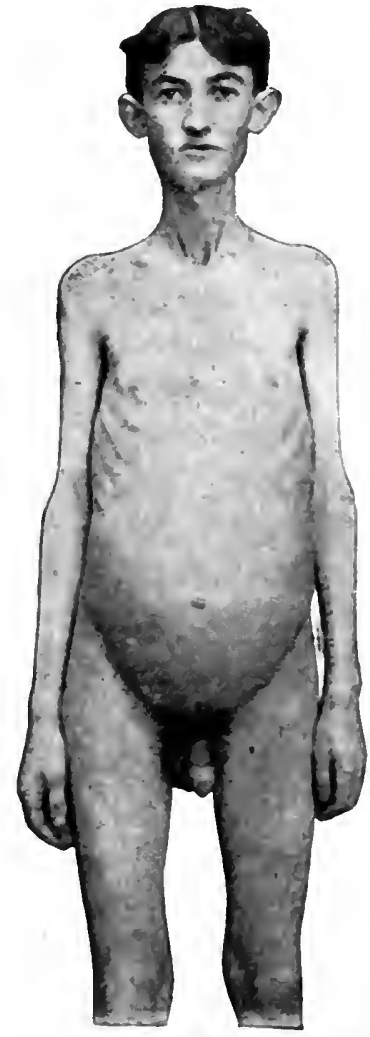

FIG. 3i.-Patient M. with Chronie. Intestinal Stenosis (Stricture of Descending Colon), showing the Barrel-shuped Abdomen. chronic intestinal stenosis is as a rule much milder than in acute occlusion of the bowel not due to a chronic condition. In the chronic form there is either no collapse at all or it is but slightly marked. Increased intestiual peristalsis is often encountered in the chronic form, especially 
during an attack of obstruction, while in the acute form this is quite rare and, if present, less pronounced. The barrel-shape of the abdomen is often present in chronic intestiual stenosis and is of diagnostic value (Fig. 36). The different forms of intestinal obstruction can be recognized by their varied symptoms which have already been described above.

Treatment.-The treatment comprises the management of the disease during the intervals and during the attacks. During the intervals the following rules are of importance: The diet should exclude all substances which give large residue of fecal matter or which are of au irritating character. Thus green vegetables, salads, fruits, vinegar, mustard, pepper, must be strictly forbidden. Milk and milk soups, eggs, tender meats without too much fat and without tendons, butter, toasted bread or plain white bread well baked, farina, rice and sago, well cooked, are permissible. The patients should eat frequently and not too much at a time. Cold drinks should be avoided. Attention must be paid to the patient's taking a sufficient quantity of food.

The bowels must be kept in working order. It is absolutely necessary to secure one evacuation daily. Massage, electricity, and the usual mild cathartics (like magnesia sulphate, rhubarb, cascara sagrada, syrup of figs) may be used. Injections of water or oil into the bowel are also of benefit. When diarrhœea is present it should not be checked unless the patient is greatly debilitated. Eren then only mild astringent remedies are permissible. Often even during periods of diarrhœa, when not very large evacuations take place, a mild eathartic (like castor oil or Carlsbad salts) must be employed in order to assure a thorough cleansing of the bowel. 
During the attack of intestinal colic warm fomentations over the abdomen should be applied. If these be insufficient, the narcotic remedies are in place. Opium alone or opium with belladonna may be given either by the mouth or in suppositories. Here also it is necessary to prodnce a sufficient evacuation of the bowel, which is best done by rectal injections. If there are great distention of the abdomen and romiting, gastric lavage is beneficial. Cocaine in doses of one-third to one-half grain, or menthol one grain three times daily, will allay the romiting. If there is a real attack of acute obstruction this must be treated in the same manner as primary acute intestinal obstruction, described above.

If the stenosis involves the upper portion of the small intestine, lavage will play an important part in allaying the symptoms temporarily. Chronic fecal impaction requires the application of massage and also of electricity, as described in the chapter on constipation. Sometimes the hardened scybala will have to be removed from the rectum by artificial means. For this purpose the sphincter is first dilated and the fecal masses are removed with the fingers or with a spoon-shaped instrument. If there is a blocking of the passage higher up in the colon, strong cathartics (croton oil) may be administered. Metallic mercury has also been advantageously used in these instances.

Strictures of the rectum, excepting those of a cancerous nature, can first be treated by dilating them gradually with bougies of various size. The rectal bougie of Credé best answers this purpose. It is advisable to leave the bougie within the stricture for at least fifteen minutes and to insert it once every two to three days. If the stricture is of a very high degree this method of treatment may be 
inefficient, and then surgical measures will have to be undertaken.

Operative Intervention.-All types of chronic intestinal obstruction, with the exclusion of those caused by fecal accumulation and strictures of the rectum, gradually grow worse. The above-described modes of treatment are only of a palliative nature. For this reason it must be considered as a decided adrance that surgical means have been found fully to remove the obstacle and restore the patient to complete health. The procedures which are resorted to are various and depend upon the anatomical lesion underlying the obstruction.

Malignant growths must be extirpated as early as possible and an end-to-end anastomosis of the bowel established. A circular stricture of the bowel (of benign type) can be removed by enteroplasty in a similar way as pyloroplasty, namely, by splitting the gut parallel to its axis or vertically to the stricture and uniting the edges of the incision transversely. Péan ' has successfully performed such operations. Several simple strictures of the bowel can be treated in the same way, if they are not too close together. If the stricture is of a tubular form or if it is of too high a degree, excision of the involved part followed by exact coaptation of the divided ends by sutures is best done. This operation is greatly facilitated by Murphy's button, which makes it possible to unite the two ends of the severed bowel rapidly without losing too much time in the suturing.

In cases in which the stricture cannot be excised nor otherwise remedied, or in any other form of obstruction of the bowel which cannot be removed, the bowel just above the stricture is united to the bowel below it and a short

'Péan: Bulletin de l'Académie de Médecine, 1890, p. 856. 
circuit thus established. This is likewise best accomplished by Murphy's button.

In some strictures of the colon in patients who are already quite prostrated, a complete operation of excision or even of the formation of a new circuit cannot be performed without too great risk of life. Here colotomy is indicated, being later supplemented by a more radical procedure when the patient is stronger and in better condition.

Adhesions should be severed and tumors compressing the bowel treated by radical removal. Surgical treatment of the intestinal stenosis, affording as it does radical relief, should be resorted to in every case as soon as the diagnosis is positive. The only excuse for subjecting the patients to non-operative measures as loug as they get along in comparative comfort, is the high mortality which surgical intervention still furuishes. According to Treves, 'the mortality fluctuates between twelve and twenty per cent. It is to be hoped, however, that owing to our advanced knowledge of this subject the diagnosis of intestinal stenosis will be made quite early, and that the patients by being operated upon at an early period will show a smaller percentage of mortality.

${ }^{1}$ Loc. cit., p. 560. 


\section{CHAPTER X.}

\section{NERVOUS AFFECTIONS OF THE INTESTINES.}

General Remarks.-The intestinal tract is rich in ganglionic cells and nerves. The plexus mesentericus Auerbach and the plexus entericus Meissner accompany it through its entire length. The vagus and the splanchnic nerves surround the intestinal canal with numerous branches and form ramifications with the ganglionic plexus., Although a thorough knowledge of the exact action of these different nerve groups has not yet been acquired, still we are certain that they govern the secretory, absorptive, and motor functions of the intestinal canal.

Secretion seems to be dependent a great deal upon the ganglionic plexus, as can be learned from Moreau's' experiment. This investigator ligated an intestinal coil and severed all the nerves belonging to it. In a few hours the coil, thus treated, was found filled with a fluid showing amylolytic qualities and containing small quantities of albumin. In order to prove the secretory influence of nerves upon the intestines. Fleischer ${ }^{2}$ justly refers to the fact demonstrated by Quincke, Demant, and Massloff, ${ }^{3}$ that in man as well as in animals after ingestion of food into the stomach,'secretion takes place in the lower part of the intestine, long before the arrival of the chyme.

1 Moreau : Centralbl. f. die med. Wissensch., 1868, No. 14.

2 R. Fleischer: "Krankheiten des Darms," Wiesbaden, 1896.

${ }^{3}$ Massloff : "Untersuchungen aus dem physiologischen Institut zu Heidelberg," Bd. ii. 
This also shows that the nerves of the intestinal tract are influenced by reflex action from the nerves or the stomach.

Vasomotor nerve filaments have also been proven to exist in the intestines. Thus stimulation of the splanchnic nerve causes a contraction, while its section is followed by dilatation of the intestinal blood-ressels. These vasomotor nerve filaments are undoubtedly also much concerned with absorption.

The motor function of the nerves and their influence upon peristalsis have been studied in an exhaustive manner by Nothnagel, Brahm-Houkgeest, and others, and have been described in the chapter on physiology (page 28).

Although under normal conditions we scarcely perceive any sensations within the intestinal tract, we are nevertheless certain that sensory filaments exist in the nervous apparatus of the intestines. This is revealed by the fact that the action of some stimuli of greater intensity than normal upon the intestinal wall, gives rise to sensatious of pain and pressure. Thus, a person not accustomed to a coarse diet, after ingestion of a large quantity of cabbage and beans, for instance, may suffer after six to eight hours from pains in the lower part of the abromen caused by the undue irritation of the small intestine. In pathological conditions the sensory character of the intestinal verves is evinced very frequently. In fact this is one of the important points which we have to consider in almost any affection of the intestinal canal.

The neuroses of the intestine may be classified into: 1 , motor neuroses; 2, sensory neuroses; and 3 , secretory neuroses. Very often these different nemroses exist in combination. Thus, a motor neurosis may exhibit features belonging to secretory or sensory derangements. The designation of the neurosis, lowever, should depend 
upon the type most prevalent. All affections of the intestines in which no anatomical lesion can be discovered are classified as neuroses or functional diseases of the intestines.

Intestinal neuroses may be primary, i.e., the affection emanates from the intestiual tract, or they may be secondary, occurring in connection with nerrous manifestations in other organs. Etiologically we know that psychical influences, mostly of a depressing nature, as fear, fright, worry and anxiety, are often the causative factors of intestinal neuroses. Neurasthenia and hysteria as well as a general neurotic tendency are also liable to produce nerrous affections of the intestinal tract. In some instances the latter conditions are due to a reflex action originating from some other diseased organ (stomach, the genito-urinary tract, uterus, etc.).

MOTOR NEUROSES OF THE INTESTINES.

Diarrhoea.

Etiology and Symptomatology.-Diarrhœa, meaning too frequent and usually too watery movements of the bowels, is always due to increased intestinal peristalsis. Diarrhcea may be the result of various morbid conditions of the intestimes, but here we shall describe the form of diarrhoea which exists without any apparent anatomical lesions.

Diarhoea may be classed under three groups: 1. Nervous diarrhœa (Trousseau).' 2. Dyspeptic diarrhœa. 3. Stercoral diarrhœa.

1. Nervous Diarrhwa. Although all the three groups of diarrhœea are primarily produced by increased peristaltic action of the bowels which is in turn caused by exagger-

I'Trousseau: "Clinique de l'Hôtel Dien," Bd. ii. 
ated action of the nervous apparatus, this group is designated as nervous diarrhœa on account of the predominance of the nerrous element. Troussean was the first to describe nervous diarrhœa. It originates either through undue stimulation of the accelerating peristaltic nerres or through some nervous influences which cause a serous transudation into the intestinal canal. Frequently both factors are probably implicated.

In many cases the stimulus may emanate from the centre and reach the iutestinal ganglia through the vagus, the sympathetic, or the splanchnic. In some cases, however, the stimulus affects the ganglionic cells of the intestinal wall directly. As characteristic instances of nervous diarrhoea we would mention those cases in which there are sereral watery evacuations after a strong emotion, thus after fright or fear. Here the stimulus arises in the brain centres supervising intestinal motions. While in these instances we have to deal with an acute transitory condition, nerrous diarrhœea can also appear in a chronic form (Nothnagel, Peyer ${ }^{1}$ ). There are persons who are attacked with diarrhoe as soon as they are in a place where a toilet-room is inaccessible. They may then be seized with abdominal pains, tenesmus, and diarrhœa. In other persons, again, the mere sight of a water-closet evokes an intense desire for an evacuation.

Occasionally the diarrhœa is preceded by several other nervous symptoms, as for instance vertigo, giddiness, congestion of the head, a sensation of heat all through the body, fright, shortness of breath, or palpitation of the heart. All these symptoms as a rule rapidly disappear after a satisfactory morement.

${ }^{1}$ A. Peyer : "Die nervösen Affectionen des Darmes bei der Neurasthenie des münnlichen Geschlechts." Wiener Klinik, 1893. Heft 1. 
This form of diarrhoea is found in persons suffering from neurasthenia or hysteria, in debilitated persons, or in perfectly healthy people after a more or less pronounced shock to the nervous system. Moreover it is met with accompanying affections of the spine. Thus Charcot described attacks of diarrhœea appearing periodically in tabes dorsalis (intestinal crises). Lastly, nervous diarrhœa may exist as a reflex condition in consequence of abnormal processes in the neighboring organs (the genito-urinary tract, uterus, etc.).

As an instance of nerrous diarrhoea the following case may be described:

N. S., thirty years old, physician, was always perfectly well. After a year of hard study and a great deal of care and anxiety he had begun to suffer from frequent loose evacuations during the last six months. As a rule the patient had one or two passages a few minutes after each meal. Preceding the evacuation rumbling noises were heard in the lower part of the abdomen, while a slight feeling of discomfort was experieuced. The movements were softer and more watery than usual, but did not contain anything abnormal (no mucus, no undigested food). The patient felt perfectly well in every respect, had a good appetite, slept. well, and had not lost weight. Examiuation of the gastric contents showed the stomach to be perfectly normal. The patient was given no medicines and was instructed to respond to nature's call in the morning and to try to suppress the evacuations after meals whenever possible. For the first few days he succeeded in having no movement after some of the meals, and gradually after a few weeks was perfectly free from the desire to evacuate the bowels after eating.

The following case is reported by Fischel : ${ }^{\prime}$

A lady, twenty-three years old, complained of a feeling

${ }^{1}$ F. Fischel : Prager med. Wochenschr., 1891. 
of oppression, cardiac palpitation, and severe attacks of diarrhoea, which appeared periodically independent of the quality of the food. The passages were watery and of a strong alkaliue reaction, smelled bad, and contained triple phosphate and considerable amounts of iutestinal enithelial cells. The examination revealed a retroflexion of the uterus. After insertion of a pessary the diarrhoea ceased.

The diarrhea appearing after exposure to cold and wet weather is most probably caused by a reflex emanating from the nerves of the skin and producing hyperamia of the intestines. The latter gives rise to transudation into the lumen of the bowel and also to increased peristalsis. This form of diarrhoea disappears very quickly (in twelve to twenty-four hours) and does not produce any anatomical changes of the intestinal walls.

Another group of diarrhœas takes its origin from an irritation of the intestinal nerres through some abnormal substances contained in the blood. The cathartic action of some remedies subcutaneously injected is the best proof of this possibility. The diarrhoea occurring in cases of septicæmia, of nephritis (with or without uræmic symptoms), and also diabetes is best explained by the theory of irritating products circulating in the blood. The diarrhœa accompanying typhoid fever and dysentery in the first stage before there has been time for the formation of ulcers, is caused by the circulating in the blood of toxic elements produced by the pathogenic micro-organisms.

2. Dyspeptic Diarhoea. Uuder dyspeptic diarrhoea may be comprised $(a)$ the diarrhcea which appears after certain articles of food; (b) diarrhoea accompanying abnormal conditions of the gastric contents.

(a) Certain foods may. cause mushy or watery evacuations, as, for instance, fresh fruit, cucumbers, cabbage, and 
beets. The liability to diarrhea from these foods, however, greatly varies in different persons. In some people milk produces diarrhœa, while in others it is rather constipating.

(b) Pronounced conditions of subacidity of the gastric contents and still oftener achylia gastrica are associated with diarrhoea. Here probably the chyme on account of its not having undergone any considerable changes in the stomach exerts mechanically too great a stimulus on the intestinal wall and thus causes the increased peristalsis. Hyperchlorhydria, althongh rarely, is also found associated with diarrhœa. Here the chyme containing too much acid most probably produces the increased peristalsis.

3. Stercoral Diarrhœea. Stercoral diarrhœa means a diarrhœa arising in consequence of too great a stimulus from fecal matter.

Etiology and Symptomatology.-If healthy persons for some cause or other become constipated for a certain period of time, the constipation may be followed by diarrhoea. The latter is generally produced by the formation of certain gases which chemically or mechanically exert a stronger stimulus upon the intestinal peristalsis. Occasionally hard scybala, as such, irritate the mucous membrane of the bowel too much and cause increased secretion and peristalsis. In stercoral diarrhœea the evacuations are as a rule at first formed and solid, later mushy and watery. Off and on these watery passages contain several small scybala as hard as a stone. Shortly before the appearance of diarrhœe the abdomen is often quite bloated and borborygmi are heard in the intestines. The patients very frequently complain of intense headaches. The passage of bad smelling flatus affords only temporary relief, while a good movement removes almost all the symptoms. Slight 
gastric symptoms may accompany this condition. A rational diet effectually arrests the diarrhoea, but after another period of constipation it may reappear, and if this happens very frequently, intestinal catarrh may be the result.

Diagnosis. - The diagnosis of nervous diarrhœa can be made, if anatomical lesions of the intestines can be excluded and if the passages do not contain a considerable amount of mucus. The special type of the diarrhoea may be determined either by the symptoms (nervous diarrhœa proper, stercoral diarrhcea) or by an examination of the gastric contents (dyspeptic diarrhcea).

Prognosis. - Most cases of nervous diarrhœa give a favorable prognosis. In some instances the diarrhoea, originally of a nervous origin, assumes a chronic course and ultimately produces an enteritis.

Treatment.-The treatment will vary according to the type of the diarrhoea. In nervous diarrhoea proper the general condition of the patient must be strengthened and the remedies will have to be directed toward this end. Arsenic and iron will often prove efficient. In some cases the administration of bromides for a few weeks will be of great benefit.

In nervous diarrhoea dependent on a reflex action emanating from some other diseased organ, the treatment must be directed toward the primary affection.

In all cases of nervous diarrhœa, persistent training of the intestines in the normal direction must be urged by the physician. The patient should be instructed after having had his first movement in the morning to refrain from any other evacuations of the bowels during this day, answering nature's call only when absolutely necessary. In quite a number of instances the patient at first continues to have 19 
the desire for an eracuation quite often, but succeeds in controlling it. Later on the desire for defecation appears less often and at last a normal state is reached.

In dyspeptic diarrhœea the treatment should be directed toward the improvement of the abnormal condition of the stomach. Thus diarrhoea due to hyperchlorhydria can be successfully checked by bicarbonate of sodium taken in halfteaspoonful or teaspoonful doses two hours after meals. The diarrhœa resulting from achylia gastrica can be remedied by a diet rich in vegetable foods, prepared in such a manner that they are easily broken up into minute particles. Stomachics, intragastric faradization, and generally the treatment of achylia gastrica will also control the diarrhœa.

In stercoral diarrhcea an efficient cathartic is the best means of checking the diarrhou. Diarrhoea having its cause in a faulty composition of the blood should be remedied by improving the constitutional condition. If this is impossible the treatment must be symptomatic.

In this connection it may be advisable to describe the means we have at our command symptomatically to treat diarrhoa, no matter of what nature it may be. The first place must be given to opium, a remedy which has stood the test of ages and is still the most reliable. It efficiently decreases the abnormal peristalsis and probably also diminishes the intestinal secretion. Morphine and the other derivatives of opium act in a similar manner, but opinm as such seems to be preferable in diarrhoeal conditions. Besides opium there is hardly another remedy efficiently to check increased intestinal peristalsis, although there are several others which may arrest the diarrhœea. Among these may be mentioned nitrate of silver, subnitrate and salicylate of bismuth, and all the remedies containing tan- 
nic acid. Another important means in treating diarrhœal conditions is heat. A hot-water bag or warm linseed poultice applied over the abdomen and warm drinks have a favorable influence upon the diarrhoea.

\section{Constipation.}

Synomyms.-Habitual constipation, atony of the bowel, constipatio, constipatio alvi, obstipatio.

Definition.-By constipation is understood a diminution in the frequency of evacuations of the bowels.

General Remarks.-Healthy persons have as a rule one evacuation of the bowels daily. Under normal conditions a movement occurs almost always at about the same time of the day. The cause of this periodicity lies most probably in nervous influences. As mentioned above in the chapter on physiology, the contents of the small intestine are propelled with comparative rapidity. In the large intestine, however, the prochoresis is very slow. The upper rectum and the sigmoid flexure form a reservoir for the storage of the fecal matter. Once in twenty-four hours through certain nervous influences the frees are carried lower down into the ampulla of the rectum and there is then experienced the desire for defecation. This is accomplished voluntarily by relaxing the sphincter ani and by exercising a moderate pressure with the abdominal walls after more or less deep inspirations. No pain is connected with this act and a rather pleasant sensation is felt after its accomplishment.

Even physiologically there is a great variability in the number of movements. Some persons have normally two or three movements a day all their lifetime, while others have only one evacuation every other day or even every three days. In both instances there may be no abnormal 
sensations whatever and we are thus forced to consider them as physiological. Constipation, therefore, should signify a condition in which a person has less frequent movements than he has been accustomed to.

In rare instances, however, the number of evacuations remains the same, but their quantity diminishes. Thus a stagnation of fecal matter in the bowels occurs (coprostasis). This condition is also usually comprised under the head of constipation. The quantity of the daily evacuation of the bowel varies greatly, depending principally upon the diet. A vegetable diet gives roluminous stools, while one consisting mainly of meats produces only a small quantity of fecal matter. The average quantity of fecal matter for twenty-four hours is about 250 c.c. While a marked divergence from the above-mentioned figure must be recognized as pathological, a small decrease of evacuated fecal matter cannot be easily discovered, the more so since, according to Woodward, a considerable quantity of the fecal matter is made up of micro-organisms whose number is apt to vary greatly, even under normal conditions.

Constipation may be due to organic lesions of the bowel (stenosis of the intestine or catarrhal conditions), or may exist without apparent anatomical changes in the intestinal tract, and thus be functional in nature. The latter class alone is dealt with here, Inasmuch as in the great majority of these cases of constipation a disturbance in the nervous apparatus of the intestine may be presumed to exist, we discuss constipation in this chapter on intestinal neuroses.

Etiology.-Habitual constipation may be divided into three groups: 1. Constipation due to retarded intestinal peristalsis (atony of the bowel). 2. Constipation due to 
a spasmodic contraction of a certain portion of intestine (enterospasmus, spastic constipation). 3. Coustipation depending upon abnormal conditions of other organs.

With regard to the etiology of the first group, namely, constipation due to atony of the bovels, which comprises by far the greater majority of cases, the following may be said: In most instances the constipation is brought on by a repeated neglect of nature's calls. Thus, young girls while in school suppress the desire for defecation out of bashfuluess, which gives rise at first to irregularity of the bowels and later on to constipation. The mental state is also responsible to a great extent for the causation of this trouble.

It is not among the working class that constipation is most frequently found, but among the wealthier classes. This shows that the mode of living has much to do with this affection. If we would go a little more into detail and try to analyze cases of chronic constipation, we would learn that the patient had perhaps at first a great deal of worry or of mental strain. At that time his bowels first became sluggish and after a while the affection became more developed. The patient experienced more and more difficulty, began to take drugs, and after a short time was not able to have a movement without medicine.

Often we find that after an acute gastric catarrh there was at first a little diarrhœe, which after a few days changed into constipation. After a short time this would have disappeared of itself, if the patient in his haste to hare a morement had not resorted to cathartics, thus upsetting again the normal state of the intestinal tract, in consequence of which chronic constipation dereloped. Very frequently the patient has some trouble, perhaps a headache, and thinks the stomach is disordered, and begius 
to live on a one-sided diet, aroids regetables, butter, fat -all substances which excite the peristaltic action of the bowels - and then constipation arises and assumes a chronic form.

In a limited number of cases the retarded intestinal peristalsis is due to a real muscular weakness of the bowel, the intestinal muscularis being much thinner than normally. Nothnagel observed some cases in which at the autopsy the muscularis of the large bowel measured in thickness 0.12 to $0.25 \mathrm{~mm}$., while normally it ought to be 0.5 to $1 \mathrm{~mm}$. In these cases the muscular development of the entire body was poor. It will therefore be easily seen that such rare conditions cannot be recognized during life.

In former years the opinion prevailed that chronic constipation gives rise to the developement of numerous nerrous affections (neurasthenia, hypochondriasis, lyysteria, and even epilepsy and paranoia). Dunin " was the first to show that in reality quite the reverse is true, namely, that constipation is the result of many nervous conditions and not their origin, for a treatment directed against the existing neurosis in many instances removes the constipation without the administration of cathartics. Dunin, however, goes too far in ascribing all cases of habitual constipation to a neurosis. There are certainly cases of chronic constipation in which no nerrous derangement whatever can be discovered.

Formerly the cause of constipation was presumed to lie in abnormal conditions of the bowels. Thus, peritonitic adhesions of the intestines and congenital malposition of the bowel have been held responsible for chronic consti-

1 Dunin: "Ueber habituelle Stuhlverstopfung, deren Ursachen und Behandlung." Berliner Klinik, 1891, Heft 34 . 
pation. But aside from the fact that these two factors are so rarely found in comparison with the large uumber of cases of constipation, Leichtenstern " proved that an abnormal position of the bowels need not cause constipation as loug as the intestiual lumen is not obstructed.

Spasmodic contraction of the bovels or enterospasmus is produced by increased peristaltic action confined to one portion of the bowels. A permanent contraction of a portion of the intestine exists which may affect both the circulatory and the longitudinal muscles. This spastic state may be of variable duration and may involve intestinal segments of different lengths. The contracted portion of the bowel is almost completely occluded, thus creating an obstacle to the onward passage of the intestinal contents.

The enterospasm may inrolve the entire small intestine. The abdomen then appears contracted in the form of a trough. This condition is met with in spinal meningitis and in other morbid processes involving the pous and the medulla oblongata. Moreover, the same affection occurs in chronic lead poisoning.

Much more frequent than the diffused enterospasm is the localized or circumscribed contraction of the bowel which usually affects a certain portion of the large intestine. Here the abdomen does not show any abnormal appearance on inspection. This condition is frequently met with in nervous people, neurasthenics, hysterical persons, and also in those debilitated by long ailments. Constipation of an obstinate nature, lasting for several dars, followed by a painful evacuation of small balls (like the

' Leichtenstern: "Verengerungen, Verschliessungen und Lageveränderungen des Darms." von Ziemssen's "Handbuch der speciellen $\mathrm{Pa}$ thologle und Therapie," Bd. vii., 2te Halfte, Leipzig, 1878. 
freces of goats) or leadpencil-shaped fecal matter are the predominant symptoms. Pains in the umbilical region or on the left side of the lower abdomen of a constricting nature and relieved after a very small passage, are also characteristic of this affection.

Constipation Depending upon Diseases of Other Organs. Numerous diseases of the stomach give rise to constipation. Foremost among these are hyperchlorhydria, ulcer and cancer of the stomach, ischochymia, atonic and catarrhal conditions of the stomach, and finally achylia gastrica - the last three, however, show. a smaller percentage of this complication. In this group of cases constipation is attributable either to the abnormal qualities of the chyme passing through the digestive canal or to the retarded gastric prochoresis or to some retarding reflex act originating in the stomach.

Tumors of the intestinal canal or of neighboring organs compressing the bowel, strictures within the intestines, and peritonitic adhesions are also often associated with constipation. These conditions, moreover, frequently lead to a far more serious concition, namely, to acute or chronic ileus. Catarrhal inflammation of the small intestines alone is also ordinarily accompanied by constipation. Ulcers of the small intestine are sometimes attended by constipation. Ulcers of the large bowel are ordinarily accompanied by diarrhoa, excepting dysenteric ulcers, which often produce constipation. Fissure of the anus and an increased contraction of the sphincter of the anus are often causes of constipation.

In many diseases of the brain, spinal cord, and the nerves (cerebro-spinal meningitis, brain tumors, hemor. rhages of the brain, chronic hydrocephalus, myelitis, tabes, neuroses and psychoses) constipation is present. 
It is due here either to a disturbance of the nervous apparatus communicating with the centre for defecation, or to a diminished sensibility of the intestinal nerves so that stronger stimuli are required than under normal conditions.

Diseases of the lungs, heart, liver, and kidney increase the liability to constipation, first, by the hyperæmia of the intestinal mucosa, and, secondly; by the congestion in the portal circulation, which both retard the peristalsis. Diabetes mellitus often gives rise to constipation, first, by the polyuria which drains the organism of water and thus leads to an exsiccated condition of the fecal matter, and, secondly, by the diet, which consists principally of meat and of a very restricted quantity of starchy food. Diarrhoea, however, is not rarely met with in this disease.

Anæmia and chlorosis are also often attended by coustipation. The latter is due to an atovic condition of the bowels, which is one of the symptoms of the general muscular atony dependent upon the impoverishment of the blood.

Most febrile diseases are also usually accompanied by constipation. Lack of exercise and an increased elimination of the fluids of the body caused by the greater activity of the lungs and the sudoriparous apparatus are the principal factors. Constipation encountered in people living in high altitudes must be ascribed, according to Ruedi,' to the same causes. The restricted diet, consisting chiefly of milk, also contributes to a lessened activity of the intestinal peristalsis.

Symptomatology. - In many cases constipation does not induce any subjective symptoms whatever. Ordinarily,

1 Carl Ruedi : "On Indications and Contraindications of High Altitude in Phthisis." The Climatologist, July, 1892. 
however, continued constipation gives rise to sensations of slight pressure, fulness and tension in the abdomen; and borborygmi may at times molest the patient. Occasionally intense colicky pains are experienced. These are due to an increased effort of the intestines to rid themselves of the accumulated fecal matter by violent contractions. The abdomen is often symmetrically distended, rarely asymmetrically, namely, in partial atony of the bowels.

In patients with thin abdominal walls, a more or less filled state of some portions of the intestine, especially of the colon, may be perceived by inspection and palpation. The appetite is often diminished and in some iustauces complete auorexia exists. Other gastric symptoms-belching, uausea, pyrosis, feeling of pressure after meals, aud bad taste in the mouth-may be present. That all these symptoms are due to the constipation and not to a separate lesion in the stomach, is proven by the fact that they all disappear as soon as efficient eracuation of the bowels has taken place.

Besides these gastric symptoms the following derangements may be present: congestion of the head, dizziness, headaches, sleeplessuess, a despondent feeling, palpitation of the heart, tachycardia, irregularity of the pulse. The latter symptoms have been considered by many writers to be due to auto-intoxication from the intestinal tract. According to the experiments of Bouchard,' howerer, this does not seem to be true, for this investigator has shown that intoxication within the intestinal tract takes place when there is a retention of fluid fecal matter, but not when the freces are solid, for in this condition no absorption of the fecal matter takes place.

1 Bouchard : Loc. cit 
Coustipation which has lasted for a long time, as a rule, terminates by a spontaneous eracuation of ordinarily very hard masses of fecal matter. The latter often appears in the form of balls which may be covered with a thin layer of mucus. In some instances the constipation terminates in an attack of diarrhoea. In these cases the diarrlioea has been caused by an acute hypersemia and inflammation of the intestiual mucosa due to the hardened fecal matter, the latter becoming liquefied through increased intestinal peristalsis aud secretion. In other instances no spontaneous evacuation takes place and it becomes necessary to make use of different cathartic remedies in order to produce a movement of the bowels.

Retention of fecal matter may cause not only a slight transient catarrhal condition of the bowels as just referred to, but may, although rarely, effect more pronounced anatomical lesions, as formation of ulcers (stercoral nlcers), local peritonitis, and even perforation of the gut with fatal issue.

One of the serious symptoms which may result from continued constipation is fecal colic. The latter begins with sudden violent pain of a colicky nature in the abdomen. In weakened persons fainting spells may occur. The abdomen is usually greatly bloated and tender on pressure. Passing of wind (flatus) gives temporary relief, but the pains soon reappear and subside only after an efficient evacuation. Fecal colic is mostly observed in cases of obstinate constipation, although it mas occur in patients with daily' eracuations of the bowels, but in these insufficient fecal passages must be presupposed. In fact, hardened balls of fecal matter can be discorered in such cases on palpation of the abdomen.

These conditions are not always of a mild character. 
As a rule cathartic remedies are efficient. In some cases, however, the latter produce energetic intestinal peristalsis and violent pains, but fail to secure a copious movement. Under these circumstances the patient may after a while sink into a state of collapse and be seized with a paroxysm of vomiting. The clinical picture now resembles very closely that of ileus. High rectal irrigations or injections of oil into the bowel ordinarily yet produce the desired effect and the patient quickly recuperates. In rare cases, however, especially in very old and cachectic persons, these means also remain fruitless. Total paralysis of the intestine now takes place and the patients are then in a most critical condition.

A frequent complication of constipation is the formation of fecal tumors. They are found most frequently in the crecum, rectum, and at the colic flexures. These masses may cause a dislocation of the colon; thus, such a tumor may be felt just above the symphysis and may belong to the transverse colon which has been dragged down to that region. Fecal tumors are as a rule easily recognizable. They are not of a very firm consistency, have a rosary-like configuration, are movable, and undergo a change in shape upon pressure. They may be of large size. Thus Levi ${ }^{\prime}$ found the rectal pouch of a patient suffering for nine years with constipation filled with a fecal mass weighing four pounds. Still larger fecal concretions have been found by Lemazurier. ${ }^{2}$ These large masses necessarily dilate the colon.

Habershon described cases in which the dilated colon measured twelve to fifteen inches in circumference, and stated that some of the normal sacculations of the colon

'Levi : Gazette méd., 1839.

${ }^{2}$ Lemazurier : Arch. gén. de méd., vol. i. 
may become distended to such a degree that they appear as true diverticula. In the latter fecal accumulations may occur which remain undisturbed by the further passage of the intestinal contents. These staguant fecal masses often produce inflammatory processes which may lead to a destruction of the intestinal coats down to the peritoneum. The colon occasionally is distended not only in width but also in length. The latter circumstance explains the abnormal position of the bowel often present in these cases.

Among the local symptoms which constipation produces hemorrhoids play an important part. They are treated in a special chapter.

A host of nerrous symptoms may develop in consequence of constipation in people who are apparently not nerrously inclined. Thus constipation lasting sereral day's may produce slight cerebral symptoms, namely, a seusation of pressure, weight and dulness in the head, sometimes headaches and vertigo. The dependence of these symptoms upon constipation is proved by the fact that after a full evacuation of the bowels they all suddenly disappear, but again return after another period of constipation. $\mathrm{Te}$ have as yet no positive explanation of the cansation of these symptoms. Some authors assume them to be of a reflex origin.

Leube ${ }^{1}$ described several cases of intestinal rertigo in which the dizziness was due to pressure existing in the lower end of the bowels, the vertigo appearing only in consequence of irritation of the intestinal walls by fecal matter or a large amount of gas, or by the examining finger. Leube concluded that pressure upon the hemorrhoidal plexuses of the sym-

'Leube: "Ueber Darmschwindel." Deutsches Arch. f. klin. Medicin, Bd. 36, 1885. 
pathetic nerve produces the sensation of vertigo in a reflex way.

Senator ${ }^{1}$ tried to explain the abore symptoms as due to the absorption of poisonons gases within the intestine, such as sulphuretted hydrogen, and Nothnagel assumed that ptomains may be absorbed and thus cause an autointoxication. But neither theory seems to hold good; for sulphuretted hydrogen gas exists in too small quantities to prodnce any marked symptoms, and the fecal ptomains can scarcely be absorbed from dried-up fecal matter.

As mentioned above, real brain diseases, hypochondria and melancholia, are never due to constipation as such. There is, however, hardly any doubt that in nervously inclined individuals obstinate constipation may be a contributing factor in the further development of some psychoses, especially melancholia.

Fecal fever, which has played a great part in the works of old writers, appears to be due in most instances not to an accumulation of fecal matter but rather to some complicating condition, an inflammatory process, a stercoral ulcer, a local peritonitis, etc. In infants and children, howerer, who much more readily develop fever, the latter may be due to accumulation of fecal matter alone. Some of the English writers have referred to chlorosis as due to habitual constipation, and Clark has treated chlorosis with cathartics. But this view has not been generally accepted and the dependence of chlorosis upon constipation is far from being proved.

Diagnosis. - The recognition of constipation is not difficult, except in those cases in which there is a daily evacuation of the bowels but not a complete one, so that fecal

'Senator: "Hydrothionæmie und Selbstinfection durch abnorme Verdauungsvorgänge." Berl. klin. Wochenschr., 1868, No. 24. 
matter is more and more accumulated in the intestine. Frequently hard fecal masses of rosary shape will be discovered on palpation of the abdomen in the region of the colon. Most often the sigmoid flexure and the capnt coli are the favored sites of this phenomenon. The detection of these fecal masses shows the existence of an insufficient evacuation of stools, in other words, coustipation.

The diagnosis of pure constipation (habitual constipation) can be made, if organic lesions of the bowels (stricture, tumor, and also intestiual catarrh) can be excluded. This diagnosis having been made, it is of importance to find out to which group the constipation belongs, whether it be due to an abnormal gastric condition, or disease of some other organ, or to a neurotic affection of the bowel itself (atonic and spastic coustipation).

Constipation due to anomalies of the function of the stomach can be ascertained only after a thorough analysis of the gastric contents and after resort to treatment directed toward the improvement of the gastric condition. Constipation due to disease of other organs (heart, luugs, kidneys, liver, etc.) may be assumed to exist when an examination discloses their presence. Constipation due to atony of the bowels is often revealed by a slightly bloated condition of the abdomen with evacuations of hard fecal matter, often balls, sometimes covered with a thin layer of mucus. While there may be a feeling of despoudency, dizziness, and somnolence, real severe pains are rare. Constipation due to a spasmodic contraction of the bowel is attended with a general feeling of uneasiness and pains in the abdomen, occasionally accompanied by fainting fits. The fecal matter is not so hard, although it is evacnated only after severe straining of the abdominal walls, and is roided in narrow tapelike pieces. The abdomen 
is often rather sunken and contracted. Intestinal coils can frequently be palpated.

Prognosis. - The prognosis of constipation is favorable in the large majority of cases, especially with regard to life. It must, however, be admitted that after having lasted a long time constipation may give rise to severe, sometimes irreparable anatomical lesions of the intestine, as for instance atrophy, peritonitic adhesions, malpositions of the bowel, even perforation with consequent peritonitis and death. The latter instances, however, are very rare, if we take into consideration the large number of persons suffering with constipation who reach an advanced age, and they will most probably become still less frequent if the patients do not neglect this condition and consult a physician at an early period.

Treatment. - Cases of constipation due to dyspeptic conditions must be treated by first ameliorating the gastric disorder. Cases of constipation secondary to diseases of other organs must be managed by first applying remedies toward the improvement of the original trouble. If these alone are insufficient, they must be managed like typical cases of habitual constipation.

With regard to the prophylaxis of constipation, we should aroid administering cathartics in slight transient disturbances of digestion and rather let nature take its own course. Never put a patient on a one-sided diet for too long a time; the exclusion of vegetables, fruits, and starchy foods in general, from the diet is frequently the cause of marked constipation. A hygienic mode of living, regular habits, less business strain and worry, and more outdoor life and exercise are of the greatest importance in the prevention of constipation.

Generally no purgatives whatever, or as few as possible, 
should be used. The chief measures in curing constipation are the following:

1. The Moral Treatment. - It is of utmost importance to allay the patient's auxiety to have a movement. He should be told to pay as little attention as possible to the condition of his bowels. Absence of a morement for a few days will cause no harm whatever. Aroidance of purgatives and keeping the mind of the patient free from worry orer the condition of his bowels is occasionally sufficient to produce spontaneous movements.

Training the patient to have an evacnation at a certain time every day is also of great importance. The patient should be taught to go to the watercloset every morning at the same time and should try to have a passage. In doing this he should not exert himself too hard and should spend only three to five minutes for this purpose. In case the attempt be unsuccessful, he should wait until the following morning, unless there is a strong desire to go to stool. Trousseau was the first to adrocate this mode of treatment, and the importance of this maxim has since been generally accepted. $\mathrm{My}$ own experience coincides with that of others, and I cannot lay too much stress upon this apparently unimportant piece of advice. Even when using other measures in combating constipation we must not lose sight of the influence in "training" the patient.

2. Dietetic Measures. - The dietetic measures have for their object the ingestion of foods which increase the intestinal peristalsis and the aroidance of substances which are of a more or less constipating nature. Adrocate the drinking of plain cold water, especially in the fasting condition, the use of buttermilk, cider, grapes, oranges, and other fruits, raw or cooked (apples, prunes, pears, peaches), lemonade, honey; salmon, sardines, herring, plenty of 
regetables, spinach, green peas, cauliflower, cabbage, green salads, rye bread, butter. Aroid strong tea, claret, huckleberries, cacao and chocolate.

Some substauces have a coustipating effect upon one person and a purgative effect upon another, as for instance milk. In treating the patient we must acquaint ourselves with his peculiarities in this respect. In prescribing a diet for patients with constipation we should allow them the usual foods with a predominance of those just enumerated. It is needless to say that some of the articles mentioned will not be permissible in every case. Thus a patient with a very delicate stomach should certainly be told not to take cabbage and cider, etc.

In some instances in which too much vegetable food has been taken and a constipation has developed in consequence of the intestine being overburdened with too much ballast, food articles containing much cellulose will have to be restricted. As a rule, however, a mixed diet with a preponderance of vegetable food is adapted for most cases.

3. Mechanical Measures.-The mechanical measures serve to strengthen the bowel and in this way promote a better action, or they directly effect a stronger intestinal peristalsis. The mechanical measures comprise massange, exercise, electricity, hydrotherapy, and lastly injections into the bowel.

(a) Massage. The general principles of massage have been described above (page 80 ). Its action consists priucipally in producing more efficient peristalsis of the large bowel. It should therefore never be used in conditions in which spasmodic contractions of the bowel may be assumed to exist. Its most useful field lies in cases of atony of the bowel.

Massage should be applied at first either by the physi- 
cian himself or under his strict supervision. It should never be applied with much force and it should nerer cause pain. According to Illoway, ${ }^{1}$ the duration of massage treatment should be from five to fifteen minutes for a grown person and from three to five minutes for children. The massage should be employed every other day with great regularity for a period of about six weeks at least. Illoway suggests that the massage sittings may be performed less frequently as soon as there is a decided improvement in the condition of the bowels. It is, howerer, never advisable to stop the massage treatment suldenly, but it should rather be kept up for a long period of time, although later at louger intervals. Massage is best applied early in the morning in the fasting condition of the patient. During its employ ment no ther remedy for constipation should be administered unless the latter has lasted several days and gives rise to varions symptoms.

Anto-massage may also be of benefit. This may be carried out by the patient himself, kneading his abdomen principally over the course of the large bowel with his right hand or by means of some instrument adapted for this purpose. Sahli was the first to recommend the use of a cannon-ball, weighing about three to five pounds. These balls may be wrapped in flannel and rolled orer the abdomen for about five to ten minutes. This procedure is best performed early in the morning in bed in the fasting condition of the patient. The ball is best rolled over the abdomen in a spiral direction, principally along the course of the colon. But the other parts of the abdomen should also be subjected to this procedure. The flannel corering the ball may be left off if desired. Dr. A. Rose, ${ }^{2}$ of New

I Illoway : "Constipation in Adults and Children." New York, 1897.

${ }^{2}$ A. Rose : New Yorker medizinische Monatsschrift, January, 1893. 
York, has practised this method quite extensively and warmly recommends its use. Dr. Arthur Kahn,' also of New York, has invented an apparatus for auto-massage which may also be used for this purpose. Rosenheim ${ }^{2}$ suggests using auto-massage in the following manner: The patient in an upright posture makes short palpating strokes with the fingers of his right hand inclined somewhat inwardly over his abdomen for several minutes. In this procedure also the course of the colon is especially to be considered.

(b) Gymnastic exercises. Exercises which bring into play especially the muscles of the abdomen are of great benefit. Exercises on the horizontal bar, horseback riding, mountain climbing, skating, rowing, bicycle riding, are all beneficial, prorided these sports are not kept up for too long a time, and do not cause a superabundant loss of water by extensive perspiration.

Indoor gymnastic exercises may also be used. Bending of the body, rotations of the trunk, especially in a siting posture, quickly drawing up the knees toward the thorax in the recumbent position, also alternate squatting and rising are of special benefit. The passive, so-called Swedish movements may also be employed either in a Zander Institute or manually by a nurse. Massage and these exercises are best applied in conjunction.

(c) Electricity. Percutaneous electrization (principally faradization) of the abdomen has been recommended by some writers as a cure for constipation. Recently direct electrization of the intestine, applying one electrode to the rectum and the other over the abdominal wall, has been

'A. Kahn : Centralblatt für Chirurgie und orthopadische Mechanik, Berlin, 1889, Bd. v., p. 4.

${ }^{2}$ Th. Rosenheim : "Krankbeiten des Darms," 1893, p. 513. 
used. Boudet's rectal electrode is best adapted for this purpose, especially when galvanization is employed. The insertion of one electrode in the stomach and the other in the rectum, as suggested by Kussmaul and Leubuscher,' has not come into use to any extent.

Electricity seems to act favorably on the intestinal peristalsis and it is especially indicated in the treatment of constipation in conjunction with massage, particularly in atony of the bowel. Doumer ${ }^{2}$ has very recently recominended the use of static electricity. He applies localized franklinization in the form of sparks or "souffles électriques "for about five to twelve minutes in the iliac fossæ, principally the left. By the employment of this method of treatment every other day for a period of two to three weeks Doumer reports having cured the most obstinate cases of chronic constipation.

(d) Hydrotherapeutic means. Hydrotherapeutic measures may be applied either alone or in conjunction with the above-named mechanical means. Hackel ${ }^{3}$ gives the following rules: In constipation due to atony of the bowels use a jet of water of about the thickness of the small finger with the force of two atmospheres, first over the epigastrium. The hose of the mobile douche is then placed over the region of the colon. Charcot's douche is best adapted for this purpose, as it allows a sudden change of temperature. When using the latter apparatus the temperature can be readily changed to any degree desired during its application. The alternations in temperature should be considerable, often from $102^{\circ} \mathrm{F}$. to $120^{\circ} \mathrm{F}$. Thus both mechanical and thermic effects come into play. After

${ }^{1}$ Leubuscher : Centralbl. f. klin. Medicin, 1887, No. 25.

2 E. Doumer et Musin : Annales d'Electro-Biologie. 1898, p. 722.

3 Jeannot Hackel : Deutsche med. Wochenschrift, Jan. 5, 1899. 
using the douche over the abdomen, it is applied over the chest and back, throwing a fan-shaped jet, the temperature being kept constant.

In constipation due to spastic contractions of the bowels Hackel applies water under a pressure of two and a half kilograms, letting it flow in the form of a fine spray. It falls like a fine rain on the abdomen. The temperature of the water should not be lower than $95^{\circ} \mathrm{F}$. and not higher than $102^{\circ} \mathrm{F}$. and should not be changed. The duration of the douche is from two to two and a half minutes. The hose is directed along the course of the colon while the water constantly runs orer the epigastrium. Ninety-six such circuits over the intestines may be made. Afterward the lower extremities, chest and back, are douched. The skin of the abdomen must not. be subjected to vigorous friction after the douche; the extremities, however, should be well rubbed. After the douche the patient should lie in bed for about five to ten minutes, being warmly covered, and then may walk for about a quarter of an hour.

Cold sitz baths $\left(12^{\circ} \mathrm{C}\right.$.) for about five minutes are also of benefit, as well as a Priessnitz compress or Neptune's girdle over the abdomen over night.

4. Injections. - Injections into the bowels of water alone or of water with the addition of soap, vinegar, common table salt, or castor-oil are often used with advantage. The amount of fluid required for a purging effect varies in different persons. As a rule a pint to a quart or one and a half quarts are necessary. These water injections should be made daily at the same hour for a period of three to four weeks, and then erery other day also for the same length of time.

Recently Klemperer ${ }^{1}$ has recommended the use of small

${ }^{1}$ Klemperer: "Therapie der Gegenwart," 1899, p. 48. 
water injections into the bowels at bed-time. Half a pint of water is injected and the patient is told to retain the fluid. The latter is rery soon absorbed by the intestine and the patient has an eracuation on the following morning. Klemperer cured cases of constipation by giving these small water injections for about three weeks every day, and then every other day for the following two or three weeks.

Injections of sweet oil into the rectum, which have been recommended by Kussmaul and Fleiner, are best adapted for the treatment of obstinate cases of constipation, especially if due to spasmodic contraction of the bowel. The injections should be made in the following way: Take about one pint of good olive oil and heat it to the temperature of the body. Then take a fountain syringe provided with a soft-rubber rectal tube, and inject the oil into the rectum. The patient takes the injection while in bed, and it is advisable to have him retain the oil as long as he can. I usually order it to be taken in the evening, so that the patient may fall asleep at once and retain it over night. The following day the oil is passed and an evacuation follows. If the patient is treated for two to three weeks with oil, the spasmodic condition will subside. The oil injections should then be given every other night for a period of two weeks, thereafter twice a week for some time, then once a week for several months.

Injections of glycerin, which were first recommended by Anacker, ${ }^{2}$ are also beneficial. Two to four grams of glycerin are dissolved in about three to four ounces of water and injected into the rectum. An evacuation of the bowels results in a very sihort time, ten to twenty

'Anacker : "Das Purgativ Oidtmann." Deutsche med. Wocheuschr., 1887, p. 823. 
minntes. The glycerin may also be given in the form of a suppository, acting the same way. While this mode of treatment is very convenient to produce an eracuation of the bowels, it should not be resorted to daily, as the bowel is thereby greatly irritated.

Similar in its action but less harmful is Flatau's ' method of applying boric acid directly to the rectum. Boric-acid powder, about one to three grams, may be inserted into the rectum with the finger or blown into it by means of a powder-blower through the anus. A movement of the bowels occurs half an hour to three hours later.

5. Purging Medicaments.-In many instances of habitual constipation the use of drugs must be resorted to. As a general rule we should administer as mild cathartic remedies as possible, and instead of increasing the dose we should rather try to reduce it gradually, and ultimately relieve the constipation without the help of cathartics.

The rarious preparations of rhubarb are very serviceable. Vinum rhei and tinctura rhei aromatica or dulcis may be griven in doses of from half a teaspoon to one teaspoonful. Rhubarb may also be given as a powder in conjunction with calcined magnesia and bicarbonate of soda, as for instance :

R Pulv. rad. rhei,

Magnes. uste, Sod. bicarb. . $\bar{a} \bar{a} 20.0(3 \nabla$.

I. f. pulv. D. ad scatulam. S. One-half teaspoonful two or three times a day.

Pulvis glycyrrhize compositus is also a very suitable preparation. It can be given in teaspoonful doses at night or in the morning. It has the following composition:

${ }^{1}$ Flatau : Berl. klin. Wochenschr., 1891, p. 231. 
B Fol. sennæ,

Rad. glycyrrhizæ ................. āā 10.0 ( 3 iiss.)

Fruct. foniculi,

Sulph. depur..................... āà 5.0 ( 3 ił

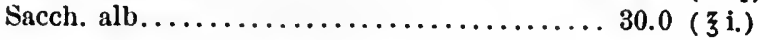

Aloes is another very efficient and popular remedy. It effects a movement of the bowels in about eight to twelve hours after its ingestion and does not cause any griping. I often give the following prescription:

R Aloes .......................... 1.0 (gr. xv.)

Extr. belladonnæ,

Extr. strychn .....................āā 0.3 (gr. v.)

Extr. et pulv. glycyrrhizæ............... q.s.

Ut f. pil. No. xx. S. One pill twice daily.

Of the newer remedies podophyllin and cascara sagrada are very valuable. Podophyllin is given in doses of onesixth to one-third of a grain twice a day. I use the following prescription:

R Podophyllin ...................... 0.3 (gr. v.)

Extr. phýsostigmatis,

Extr. nuc. vomic................. āa 0.5 (gr. viiss.)

I. f. cum extr. et pulv. glycyrrhize q. s. pil. No. xxx. S. One pill twice daily.

Cascara sagrada may be given in the form of fluid extract, fifteen to twenty-five drops twice daily, or cascara sagrada with maltine, one teaspoonful once or twice daily.

Syrup of figs, one teaspoonful at night time, or tamarind, also one teaspoonful, is often of value.

Jalap and colocynth belong to the stronger drastic remedies, and hardly ever find a place in the treatment of the cases under consideration. Hunyadi Janos water, Friedrichshaller, Homburger or Rakoczy waters, Apenta, Rubinat and the like are also sometimes of benefit. They should, however, not be used for a long period of time in cases of anromia and neurasthenia. 


\title{
CHAPTER XI.
}

\section{NERVOUS AFFECTIONS OF THE INTESTINES.}

\author{
MOTOR NEUROSES.-(Continued.)
}

\section{Paralysis of the Intestines.}

Partial paralysis of the intestines may occur and give rise to symptoms resembling a complete occlusion of the intestinal lumen. Paralysis arising in consequence of a mechanical obstacle to the passage of the intestinal contents has been described abore. Here we shall deal with primary paralysis of the intestine without any organic obstacle. In this condition the peristaltic motion of this organ is absent. The passage of fecal matter is thereby interrupted and symptoms of obstruction result.

Henrot ' distinguishes three forms of intestinal paralysis:

1. Direct paralysis of a portion of the intestine caused by alterations of its walls. Thus an intestinal coil may become paralyzed after repeated forced reposition of a hernia or after it has been incarcerated in the hernial pouch for a long time. The paralysis may also occur as a consequence of a direct trauma or after extensive abdominal operations, and finally after various chronic inflammatory and ulcerative processes of the intestine (enteritis, tuberculosis, dysentery).

2. The paralysis is caused indirectly in consequence of

'Henrot: “Des Pseudo-étranglements," Paris, 1865. 
a reflex nervous action. Thus contusion of the testicles, inflammation of a hydrocele, abscesses of the abdomen may inhibit the abdominal nerve centre in such a way that the peristalsis ceases, although this is of very rare occurrence.

3. The intestinal paralysis may result from general neuroses (hysteria), from psychoses (melancholia, hypochondria), or from affections of the central nerrous system (meningitis, brain tumors, tabes dorsalis, myelitis, etc.).

Besides these three groups, which are all of a more or less acute character, Rosenheim also mentions paralysis of the intestines as a consequence of coprostasis due to atony of this organ, which condition is less acute and more protracted. The patient, as a rule, has suffered from constipation for a loug time. Evacuation of the bowels has been artificially produced only after the appearance of many annoying symptoms. At last the usual remedies refuse to work and the patient now becomes a chronic sufferer. Dyspepsia, intense meteorism, and palpitations of the heart are present. The ingestion of food grows smaller every day and the patient becomes weaker. This condition may last for weeks and months, and if no radical remedies are resorted to, the patient may ultimately be seized with fecal vomiting and die of the intestinal paralysis.

According to Rosenheim, a sudden attack of serious intestinal obstruction in a patient suffering from chronic constipation is, as a rule, not caused by paralysis of the intestine, but rather by an occlusion of the intestinal lumen through hardened fecal matter.

The diagnosis of intestinal paralysis can be made if all the other numerous factors causing ileus can be excluded and one of the above-mentioned etiological points can be discovered.

The treatment of these cases cousists in the applica- 
tiou of electricity (recto-abdominal galvanization), massage, and purgative high rectal injections. Cases in which the paralysis is caused by chronic constipation must be treated by high injections of either ice water two hundred to five hundred grams, or water with the addition of two hundred to five hundred grams of oil. These injections should be applied twice or three times a day for several days in succession until a satisfactory result has been obtained. Massage aud electricity can be used in addition to these injections. Internal purgatives, even croton oil, are not efficacious in this class of cases. The use of mercury, however, in doses of three hundred to eight hundred grams is here of great value. In cases in which the lower part of the colon is the seat of the paralysis, the stagnant fecal matter must be removed with the hand before the rectal injection is resorted to.

\section{Proctospasmus, or Spasm of the Rectum.}

This condition consists in attacks of painful contraction of the sphincters of the rectum and is in most instances a secondary affection. It is mostly found in inflammatory and ulcerative processes of the rectum and colon, in fissure of the anus, and also in inflammatory diseases of neighboring organs, bladder, prostate, nterus.

Spasm of the rectum may, however, occur also independently as a primary nervous affection. As such it is principally met with in individuals with a nervous taint, and in diseases of the spinal cord. The attacks of proctospasmus differ in intensity and also in duratiou. Sometimes they last only a short while, a few minutes, sometimes several hours or even days. In the milder form defecation is accompanied by intense pains and takes place only after great effort. In the severer forms there is a strong desire 
for defecation, but notwithstanding the most intense pains and great straining there is no movement of the bowels. If these attacks last several hours they greatly weaken the patient and render him very despondent. The anus is very sensitive to touch, and a digital examination of the rectum during the spasm is hardly erer possible. A thorough examination of the rectum can be made only during anresthesia. In instances of very severe proctospasmus a tran. sient paresis or paralysis of the sphincter muscles may result.

The diagnosis of proctospasmus is easy, as the srmptoms are very distinct. The diagnosis of the primary nervous form will be made if organic diseases of the rectum and of the neighboring organs can be excluded.

The treatment must be directed principally toward the primary affection. In cases of nerrous proctospasmus the treatment should be symptomatic and consist in the use of narcotic remedies. In severe forms of this malady hypodermic injections of morphine must be resorted to. In some instances a forcible divulsion of the sphincter under chloroform narcosis may become necessary.

Paresis and Paralysis of the Sphincters of the Amus.

Paralysis of the anal sphincters occurs frequently in consequence of long-lasting affections of the rectum. Sometimes orer-exertion of these muscles (tenesmus) ultimately leads to exhaustion. Occasioually ulcerations and infiltrations of the rectum involve also the sphincters or entirely destroy them, thus annulling their functions. The tonicity of the sphincter muscles may be impaired in persons who have suffered for a long time from an accumulation of fecal matter in the lower portions of the bowel. The muscular apparatus being over-irritated for a long time becomes 
weakened and exhausted. Diseases of the brain and spinal cord, leading to inhibition of the will power, may likewise canse paralysis of the sphincters.

Atony, paresis, and paralysis form different degrees of this affection. Some patients are not able to keep the rectum tightly closed, and a small amount of secretion continually penetrates through the anus. After defecation they have the sensation of not having finished the act. Sometimies there may be an involuntary movement of the bowels in consequence of the loss of the contractile power. This, however, occurs only after strong excitement, intense bodily exertion, during urination, and rarely in walking.

In case the paralysis of the sphincters is complete, flatus and fecal matter will escape involuntarily even in a state of rest. In paralysis resulting from proctitis, hemorrhoids, stricture, etc., there is a continuous dripping of a mucosanguinary secretion which greatly irritates the skin surrounding the anus.

Diagnosis. -Paralysis of the anal sphineter can be recognized rery easily. The anus appears patulous and the anal folds have disappeared. Two and even three fingers may be introduced into the rectum without encountering any resistance. In making the diagnosis of a purely nervous paralysis anatomical lesions must first be excluded. This is done by means of a thorough examination of the rectum with a speculum.

Prognosis. - Paralysis resulting from anatomical lesions of the rectum gives an unfavorable prognosis. In the purely neurotic form, however, the prognosis is much better.

Treatment. - It is of great importance to secure a thorough evacuation of the bowels, which is best done by rectal injections of water twice daily. Paralysis due to fecal impaction' as such, can be entirely remedied by the just 
mentioned measures aloue. Thus Wallace 'reports a cure in a case of a nine-year-old boy who was troubled for three years with incontinency of freces, which dropped out whenever he walked. The patient was treated with water enemas to which castor oil had been added, and later ou with injections of water with the addition of alum. After a month's treatment hie entirely recovered.

In paralysis clue to affections of the central nerrous system electricity and massage may be of benefit. Hypodermic injections of strychnime (0.001 to 0.0015 pro dose) into the anal folds have been recommended by Rosenheim. Cases in which the paresis of the sphincter is due to a difficulty in urination and a continuous straining in order to void the bladder, the paresis will be improved by artificially emptying the bladder by means of a catheter for a considerable length of time.

Peristaltic Restlessness of the Intestines.

Definition.-Increased peristaltic motions of the intestines in such a way that they become visible through the abdominal walls.

Etiology and Symptomatology. - While in the normal state intestinal peristalsis is accomplished without being visible or making itself felt, in pathological conditions increased peristalsis may exist which can be easily percoived throngh the abdominal walls and which is usually accompanied by distinct noises (borborygmi). Increased intestinal peristalsis may accomjany any complete or incomplete occlusion of the intestinal lumen or it may be caused by purely neurotic influences. Here only the latter form is dealt with, as the former condition is discussed in connection with the organic lesions causing it.

${ }^{1}$ Wallace: St. Barthol. Hosp. Report, 1888. 
Usually peristaltic restlessness of the intestines occurs in the form of attacks, lasting several hours in succession and reappearing after more or less long periods of time. In some instances the patients complain of various movements and noises within the abdomen due to the increased intestinal peristalsis, while pain is absent. In other instances the above sensations are now and then interspersed with serere colicky pains. The majority of cases of peristaltic restlessness of the intestines is accompanied rather by constipation, seldom there are either normal evacuations or diarrhoea. In some instances the exaggerated peristaltic motions continue eren after intestinal digestion has been completed, and are accompanied by painful sensations. Peristaltic restlessness of the intestines is occasionally associated with peristaltic restlessness of the stomach.

Peristaltic restlessness of the intestines is principally met with in nervous persons, in the hysterical and hypochondriacal. Occasionally, however, it occurs in persons who do not present any other nerrous symptoms. In women this condition may exist during the monthly periods or pregnancy. In some persons it appears after the ingestion of highly spiced or indigestible foods, after the excessive use of tobacco, after great psychical excitement or too much brain work. In other cases, howerer, none of these etiological factors can be discovered.

Diagnosis. - The diagnosis of peristaltic restlessness of the intestines is made whenever pronounced intestinal motions are visible through the abdomen. The nervous character of this condition is recognized, first, after exclusion of organic affections of the intestines; secondly, by its periodic appearance.

The prognosis is favorable.

Treatment. - In the first place it is of importance to in- 
vigorate the entire organism and especially improve the condition of the nervous system. With regard to diet sufficient quantities of food should be given, but too spicy and indigestible nourishment should be excluded. In cases accompanied by some abnormality of the bowels their function should be regulated. The bromides, valerian, and asafetida are of decided value. Drinking of warm water or tea and hot applications are useful during the attack. Arsenic alone or in combination with iron is of benefit in cases combined with anæmia. If the condition assumes a violent character and is accompanied by serere pains, a small dose of an opiate, alone or in combination with belladonna, is appropriate. If the intestinal restlessness appears at night time and prevents the patient from sleeping, chloral hydrate, sulphonal, or trional may be administered. Electricity and massage of the abdomen have been variously recommended, but neither of the two ap-. pears to me to be of great value in this condition. Change of climate and surroundings is often of benefit.

\section{Meteorism.}

Meteorism, tympanites, or flatulency signifies a condition in which there is an excessive accumulation of gas in the intestinal tract.

If not caused by an organic obstruction in the bowel this condition is due to an abnormal state of the intestinal motion and absorption. Owing to the first factor we describe this anomaly under motor neuroses.

Etiology. - The causes of the excessive accumulation of gas are: 1 , an increased ingestion of gases themselves or of substances which easily form them; and 2, a diminution or impairment of their elimination from the intestines. The increased introduction of gases may cousist in 21 
swallowing of air or in drinking beverages highly charged with carbonic-acid gas. In both instances the gases principally accumulate in the stomach, although a portion of them reaches the intestines.

Increased formation of gas within the intestine is the consequence: 1 , of varions processes of fermentation and disintegration of carbohylrates and fats, hydrogen aud carbon dioxide being the principal gases; 2 , of the decomposition of proteids which produce besides the gases just mentioned sulphuretted hydrogen, carburetted hydrogen, and methyl mercaptan. The increased formation of gas is mostly due to an increased ingestion of easily fermenting food.

A diminished elimination of the gases may be due : 1, to an inhibition of the passage of the flatus; and 2 , to retarded absorption. The passage of flatus is inhibited either by an intestinal obstruction or occlusion, or by a paresis or paralysis of the intestinal muscles. The latter condition is found in peritonitis and in grave infectious diseases, after shock, in severe anæmia, in spinal affections, and also in general neuroses. Most cases of meteorism, which quickly appears and just as quickly leaves the patient, are due to paresis of the intestinal walls and are usually associated with a large number of other nervous symptoms.

Symptomatology. - A certain degree of tension about the abdomen, more or less pronounced, is almost always experienced by the patient. In some instances the abdomen protrudes in balloon-shape, the region of the navel being principally involved. This picture is mostly met with in patients with relaxed abdominal walls. In cases in which the latter are tense the accumulation of gases may push the diaphragm upward. Sometimes the lungs and heart are forced upward and severe dyspnoea develops, which 
in rare instances may be followed by asphyxia, collapse, and even death. There is a coustant feeling of pressure and a desire to pass wind, while colicky pains are also occasionally met with. As a rule no flatus can be passed or very inconsiderable amounts at long intervals.

Differing from the form of meteorism just described are those cases in which there is a slight tension over the abdomen and wind is passed from the anus almost constantly for a long time with much noise. It is lighly improbable that the gases emitted in this variety of cases are really produced in the intestinal tract for the following reasons: 1 , there is no considerable change in the size of the abdomen after a repeated passage of considerable amounts of gas from the anus; 2 , the absence of relief felt by the patient after the passage of flatus; and 3, the almost odorless character of these gases. Rosenheim compares these cases with those of nervous eructation from the stomach. While in the latter the air is constantly swallowed by the patient and belched up again, in the intestinal variety Rosenheim assumes that the air is constantly pumped into the rectum in order to be again emitted as flatus.

The diagnosis of meteorism is made whenerer an excessive amount of gas is discorered in the intestines.

The prognosis will depend upon the cause which creates the tympanites. If it be clue to organic lesions of the intestines (occlusion of the lumen) the prognosis is very grave, while meteorism due to a purely nervous disturbance gives a favorable outlook.

Treatment.-In instituting a curative plan for this affection it will be necessary to elucidate the etiological factor of the meteorism. If the latter is caused by an obstruction of the bowel, this primary affection will have to be treated as such. In most instances of meteorism of neu- 
rotic origin the following points are of importance: Drinks and foods containing or forming a large amount of gas should be prohibited; thus all carbonated waters, beer, champagne, and cider should be aroided. Fresh fruits, all kinds of cabbage, leguminous foods, potatoes, coarse rye bread, sweetened cake, rich gravies should be carefully abstained from. These rules apply not only when the metoorism is fully developed but in patients with a disposition to flatulency.

Formerly numerous intestinal antiseptics were given with the object of lessening the fermentative processes in the bowels. Recently, however, the general view prevails that they are of no benefit whatever. Benzonaphthol, salol, and salicylate of sodium are still regarded as the most efficient in this respect and may be tried in suitable cases. They can perhaps be advantageously administered in Sahli's ' glutoid capsules in order to prevent their absorption in the stomach. Calcined magnesia, lime water, charcoal, testa præparata, and subnitrate of bismuth are given with the intention of absorbing the gas, although their actual effect in this respect can naturally be only very limited.

The following drugs are believed to have a beneficial influence in diminishing the gas, especially in mild forms of flatulency : poppy-seed, peppermint, spearmint, thyme, cinnamon, cloves, nutmeg, anise, fennel. These are best given in infusions. It has not as yet been scientifically proven whether the reaction following their administration is due to a slight increase of the intestinal peristalsis. Brunton and $\mathrm{Cash}^{2}$ are of the opinion that the carminatives, such as asafetida and oil of cloves, have a distinct.

1 Sahli : Deutsche med. Wochenschr., 1897, No. 1.

' Brunton and Cash : St. Barthol. Hosp. Report, 1887. 
effect upon the absorption of sereral gases (carbonic-acid gas and sulphuretted hydrogen).

The removal of the gas per vias naturales through the anus is the most efficient therapeutic measure. This can be done through cathartic remedies whenever there is no contraindication against their use. Large cleansing enemas of water, with the addition of a teaspoonful of essence of peppermint or oil of turpentine emulsified with an egg to a quart, are of benefit.

When there are no anatomical lesions, massage of the abdomen and faradization may be of advantage. This also applies to friction of the abdomen with a. cloth dipped in some alcoholic solutions of aromatics or ethereal oils (linimentum saponis, oleum carvi, cajuputi, terebinthinæ, etc.). The introduction of a tube into the rectum may be helpful in favoring the escape of gas from the lower parts of the colon. In desperate cases in which the meteorism has reached such dimensions as to endanger life, puncture of the intestine through the abdomen with the trocar has to be resorted to. The meteorism of hysterical persons often requires no treatment, as it usually disappears of itself. Sometimes, however, it is very obstiuate to all therapeutic measures.

\section{SENSORY NEUROSES OF THE INTESTINES.}

While normally no sensations originate in the intestinal canal which become perceptible even during digestion, in pathological conditions this organ may be the seat of the most painful feelings. The latter originate in the fibres of the sympathetic nerve. Most of the sensory neuroses of the intestine consist in an increased excitability of the sensory filaments of these nerves. There are, however, a few 
conditions in which a lessened sensibility exists. The latter relates principally to the sensory nerves of the rectum. Normally the entrance of fecal matter into the rectum mechanically irritates these nerves and creates a desire for an evacuation, while a lessened irritability of the rectal nerves may fail to produce the above sensation.

\section{Enteralgia.}

Synonyms.-Intestinal colic. Neuralgia mesenterica.

Definition.-Pains in the intestines.॰

Etiology.-Enteralgia is present in most organic lesions of the intestines. Enteralgia of purely neurotic origin, however, which is considered in this chapter, occurs indepently of any anatomical lesious of the intestinal walls.

Sometimes abnormally strong stimuli may be erolred within the intestinal canal, producing painful seusations. These stimuli may be of a mechanical, chemical, or thermal character. Thus, a conglomeration of intestinal worms, foreign bodies, gall stones, or enteroliths may produce intense colic. Sometimes hardened fecal masses press upon the sensory nerves. The intestinal lumen being temporarily occluded by these masses, gases collect above this space and increase the tension within the intestinal canal, thus giving rise to intense pain (wind colic, colica flatulans, which is quite often seen in children). Sometimes the ingestion of very coarse foods, indigestible substances, tainted foods, too cold beverages, highly fermented drinks cause enteralgia. In the gouty diathesis it may precede a gouty attack or replace it. Similar to these conditions in which the enteralgia takes its origin from toxic substances contained in the blood and irritating tho intestinal nerres, is also the intestinal colic met with in chronic iutoxication from lead or copper. 
Aside from these forms of enteralgia due to a certain discoverable irritating factor, it may also result from a perrerted state of the sensory intestinal uerves themselves. The latter group is principally found in patients affected with hysteria, or spinal troubles, although it may also be of a reflex nature due to abuormal conditions of neighboring organs, kidneys, bladder, uterus, oraries, and liver.

Symptomatology. - The symptomatology of enteralgia presents quite a varied picture, in many instances depending upon the cause of the enteralgia. If the neuralgia mesenterica is due to an error in diet, it usualls begins with gastric disturbances, belching, nausea, romiting, and anorexia. In cases in which an accumulation of fecal matter produces the enteralgia, obstinate constipation and flatulence, occasionally alternating with diarrhoea, precede the attack. In chronic lead poisoning there are present a bluish line around the gums near the teeth, retarded pulse, and oliguria.

The principal symptom of neuralgia mesenterica is pain within the intestine. It seldom appears suddeuly and with great violence. As a rule, the pains are at first of light character and gradually increase in intensity. They are of a cutting, throbbing, or pinching nature, and are experienced usually in one and the same abdominal area, most often in the region of the navel. Starting from this spot they radiate toward the back, the loins, the thighs, and the testicles. In some cases the pain wanders from one area to another and may be felt at different times in the most raried regions of the abdomen. In the latter instances the pains are accompanied by a visible peristaltic restlessness of the intestine, often producing gurgling noises.

In mild cases the pain is quite endurable, and often lasts 
but a short while. In severer forms of enteralgia, however, the pains may be of extreme violence, and in weak patients may produce syncope, while in the more robust thes may give rise to attacks of panting and crying. The face grows pale and assumes an expression of intense suffering. The forehead is covered with cold perspiration and the extremities are cold. The entire picture resembles very much that of shock.

Pressure in many instances slightly alleviates the pain, and for this reason the patients often press their hand or some other hard substance against their abdomen. For the same reason they are often found lying on their abdomen, pressing the latter against the mattress. In cases, however, in which the intestinal tract is filled with gas and the abdomen therefore in a tense condition, even very slight pressure increases the pains. Under these circumstances a suspicion of peritonitis often arises. Ultimately the pains gradually decrease, and disappear much quicker if the accumulated fecal masses and gases have been evacuated spontaneously or by means of injections. The attack is then orer:

Spastic contractions of the intestine are often encountered, especially when the pains are of intense character. If these contractions involve a large part of the intestine, as is often the case in lead colic, the abdomen appears trough-shaped. The abdominal walls are guite tense and often very rigid. In case the spasms are limited to isolated intestinal coils, the abdomen is not drawn in and at some places where there are intestinal coils overfilled with fecal matter and gas, may asymmetrically protrude. In stercoral and wind colic the abdomen usually is tympanitic.

Constipation is almost always present. Frequently there is also a retention of the intestinal gases. If the lat- 
ter are passed in considerable quantity, the pains often subside for a short while, or, in some instances, especially in the so-called wind colic, entirely disappear.

The intensity and the duration of the attack are subject to great variations. It may last from a few hours to several days. The pains are sometimes but very slight, and again of such violence that even large doses of opium are hardly effective.

Aside from the above-mentioned symptoms there exist quite often shortness of breath, palpitations of the heart, a sensation of oppression, tenesmus, strangury, hiccough, vomiting, seldom pollutions and priapism. Occasionally cramps of the calves and even general convulsions are observed. In cases of hysteria hy perasthesia of the abdominal walls is encountered.

Diagnosis.-Enteralgia is easily recognized when it presents the above-described characteristic picture. Its neurotic nature, however, will be inferred from the following features: It appears in attacks, and subsides suddenly. There are almost alwars other nervous symptoms present. In enteralgia due to anatomical lesions of the intestine the pain is, as a rule, increased by pressure upon the abdominal walls. Another distinguishing mark for the latter is that it is more often accompanied by diarrhœea, and that the dejecta contain pathological admixtures (blood, mucus, rarely pus).

With regard to the differential diagnosis the following conditions which are accompanied by abdominal pains will have to be excluded: Rheumatic affections of the abdominal muscles, lumbar abdominal neuralgia, hyperæsthesia of the abdominal walls, peritouitis, biliary and renal colic.

Rheumatism of the abdominal muscles is characterized by the following features: The pain is situated orer the 
superficial area and not within the abdominal cavity. It often changes its seat. It is of longer duration than enteralgia and does not show any distinct exacerbations nor diffusion. Pressure increases the pain, while rest in a recumbent position eases it. Anti-rheumatic remedies (salol, sodium salicylate, salipyrin) subdue it.

In lumbar abdominal neuralgia the pain is localized on the surface and limited to one intercostal space which is rery painful to pressure. The pains often radiate to the back, the hypogastrium, and the genital organs. Antineuralgic remedies (antipyrin, antifebrin, phenacetin) are often efficient.

Hy perresthesia of the abdominal wall is, as a rule, met with in hysteria and neurasthenia. The pains are localized in the superficial layer. The slightest touch of the skin of the abdomen increases the pain. The faradic current often quickly removes it.

In peritonitis there is almost always fever, and the pain is increased on pressure. Meteorism is here much more frequently encountered than in intestinal colic. Frequently dulness in the lower part of the abdomen (exudation) is observed.

Biliary and renal colic are recognized by the situation of the pain which often corresponds to the location of the affected orgau. Besides, other symptoms are usually present which are characteristic of the latter (icterus, strangury).

Prognosis. - The prognosis of intestinal colic is almost always good with regard to life, for the attack usually ends in recovery. Exceptioual cases of death have, however, been observed by Oppolzer ${ }^{1}$ and Wertheimer. ${ }^{2}$

Treatment.-The treatment consists, first, in measures

1 Oppolzer: Wiener meā. Wochenschr., 1867.

2 Wertheimer: Deutsches Arch. f. klin. Medicin, 1866, Bd. 1. 
directed toward the removal of the cause, and secondly, toward the relief of the pain. In most cases of intestinal colic a thorough evacuation of the bowels is of benefit. For this purpose injections of a considerable quantity of water (one to two quarts) or of olive oil (one-half to one pint) are very serriceable. Mild cathartic remedies, castor-oil, calomel, and the like, may also be administered. In cases in which worms have been found a rermifnge must be given with the cathartic. If meteorism is quite pronounced massage of the abdomen may be tried. If the colic is due to an error in diet, the latter must be strictly regulated. If due to a general cold, hot bererages (tea, infusions of camomile and of peppermint), hot poultices over the abdomen are of value.

In nerrous enteralgia occurring in patients suffering from hysteria and neurasthenia the treatment should be directed toward the improvement of the latter conditions. Climate, electricity, massage, and hydrotherapy play a predominant part here.

The following symptomatic measures which serve to subdue the pains are of great importance: If the colicky pains are quite serere, the administration of an efficient dose of an opiate is indicated. Tincture of opium may be given in doses of fifteen or twenty drops, or opium extract, 0.03 to 0.05 ; or morphine, 0.01 to 0.015 , may be injected subcutaneously. Even in cases in which the colic is due to a retention of fecal matter, the narcotics just mentioned are indicated, for they relieve the spastic contractions of the intestines.

During a serere attack of intestiual colic the diet should consist principally of liquids, small quantities of milk and broth being given at frequent intervals (about erery two hours). If the attacks recur quite often, the application 
of the galvanic current (one electrode within the rectum, negative pole, the other over the abdomen) is sometimes of benefit.

\section{Hypogastric Newalgia.}

(Neuralgia hypogastrica, Romberg. ${ }^{1}$ )

Enteralgia limited to the lower portion of the large bowel is termed hypogastric neuralgia. In this condition there exist disagreeable, sometimes painful sensations in the lower region of the abdomen and in the lower parts of the back, accompanied by a violent feeling of pressure in the rectum and sometimes also in the bladder. In female patients the same sensation may also extend to the uterus and vagina. Sometimes the patient also complains of painful sensations in the perineum and the thighs. Persons suffering from hemorrhoids and women aftlicted with nervous and uterine troubles are principally liable to suffer from this condition. This form of neuralgia is also frequently found in diabetic patients. Sometimes the patients have the sensation as if a foreign body were in the rectum.

The treatment resembles very much that of intestinal colic. The original trouble predisposing to hypogastric neuralyia should always be first treated. If congested piles are present, application of leeches about the anns and warm sitz baths must be recommended. If the pains are violent, suppositories of opium alone or with belladonua should be used. The diet should be a bland one and the bowels should be carefully regulated.

1 Romberg : "Lehrbuch der Nervenkrankheiten," Berlin. 


\section{Hypercesthesia, Parcesthesia, and Ancesthesia of the Intestine.}

While in the normal state no disagreeable sensations are manifested during the act of intestinal digestion and defecation, in some cases of neurasthenia or hysteria we meet with exceptions to this rule. Thus, even without apparent anatomical lesions of the intestine, there may be a seusation of pressure, fulness, of pinching, of heat or cold in the lower region of the abdomen a few hours after the ingestion of food. The same sensations may also occasionally appear without the patient haring eaten anything, after bodily exertion and excitements, especially after sexual intercourse.

The rectum and the auns are particularly liable to be the seat of abuormal seusatious. Plyysiologically a feeling of fulness is experienced in the rectum when the fecal mass has accumulated in this locality. In case of neurasthenia a sensation of fulness with an inclination to go to stool may appear, even when the rectum is entirely empty. Sometimes a feeling of pressure or weakness in the anal region may be present; sometimes the patient may be tormented by a constant burning or itching in the same region. The act of defecation may be accompanied by erections, sometimes by a feeling of uneasiness; quite often a feeling of extreme fatigue after defecation is experienced.

Ancesthesia of the rectum is observed in the same class of patients. The seusation of fulness in the rectum, which causes the desire for defecation, is then absent; there is, therefore, never a desire for eracuation. In very prononnced cases of rectal anæsthesia it mar occur that even the passage of fecal matter through the anus is not felt. Such a 
high degree of anrsthesia, however, is met with only in patients with spinal and brain troubles and in very old and lecrepit individuals. Paralysis of the sphincters, which has been described above, may occasionally accompany the anrsthesia of the rectum and thus aggravate the latter. In such instances involuntary evacuations of the bowels take place without the patient's knowledge. He becomes aware of this fact only after his clothes have been soiled and by the fecal odor.

In the treatment of these abnormal sensations within the intestines attention must be directed toward the improve-

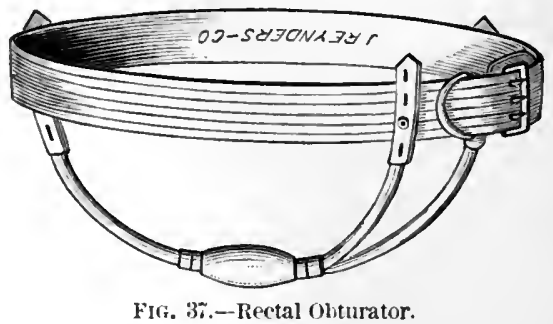

ment of the general condition, thus raising the nervous tone of the organism. Hydrotherapeutic measures and climatic influences are of the greatest importance. While dietetic measures as such are without much influence upon the nervous disturbances which appear during the intestinal digestion, spicy food and alcoholic beverages sloould, notwithstanding, be forbidden and an essentially vegetarian regimen recommended. The abnormal sensations within the rectum and anus may be improved by cooling rectal douches, by sitz baths, and also by rectal galvanization.

In cases of anrsthesia of the rectum a cleansing enema in the morning will remove the fecal matter and thus be beneficial during the day. Patients suffering from the 
severer forms of anresthesia should wear a rectal obturator held in place by means of a T-bandage during the day (Fig. 37).

\section{SECRETORY NELROSES OF THE INTESTINES.}

Although there is no doubt that secretory nerves exist in the intestines-for it has been shown that the entrance of food into the stomach is immediately followed by secretion not only in the small intestine but also in distant parts of the large bowel-still we are yet very far from the knowledge of their exact location. Nervous diarrhoea, which has been described under the motor neuroses, is often accompanied also by an increased flow of intestinal juice. Conditions in which there is a lessened secretion of intestinal juice are not yet positively known. It may be that they exist in cases of constipation, being perhaps the cause of the latter in some instances. While, howerer, in the disturbances just mentioned the increase or decrease of intestiual secretion is a mere hypothesis, one affection of the intestines exists in which increased secretion is positively found. This is the so-called membranous enteritis.

\section{Membranous Enteritis.}

Synomyms.-Mucous Colic; Tubular Diarrhœa; Membranous Diarrhoea.

Definition.-By membranous enteritis is understood an affection in which more or less large pieces of mucus (usually ribbon-like) are passed periodically with the freces.

History. - This affection seems to have been familiar to the medical world for several centuries. Paulus Egineta, in speaking of the passage of the inner membrane of the

1 Paulus Agineta : Cited from Da Costa, American Journal of the Medical Sciences, 1871, p. 321. 
intestine, has certainly dealt with cases of membrauous enteritis, and erred only in the explanation of these masses.

Sennertius and Morgagni' 'recognized these membranes as mucus, which had been inspissated and moulded in the intestine.

Mason Good ${ }^{2}$ was the first to describe this affection under the name of "tubular diarrhoea," which name has also been accepted by Woodward. "The latter author adds that in case the membranes in a given instance have no tubular form, the expression "membranous diarrhœea" is suitable.

F. Siredey ${ }^{4}$ contributed a very valuable paper in 1869 in reference to the knowledge of this affection. He described one case of mucous discharge in a man and six cases in women, and arrived at the conclusion that in some instances these mucous discharges occur in patients whose intestinal tract does not reveal any organic lesion whatever. For this reason Siredey regards this affection as an intestinal neurosis, occurring principally in hypochondriacs and hysteries.

Whitehead ${ }^{5}$ describes this affection under the name of "mucous disease," cites the entire old literature, and gives detailed rules with regard to treatment and diet. $\mathrm{He}$ says: "Exercise, short of fatigue, should be taken daily.

'Sennertius and Morgagni : Cited from J. G. Woodward, "The Medical and Surgical History of the War of the Rebellion," 1879, part ii., vol. i., p. 363.

"Mason Good: "The Study of Medicine," cl. 1, ord. 1, species 7, vol. i., Philadelphia, 1825, p. 162.

${ }^{3}$ Woodward : Loc. cit.

${ }^{4}$ Siredey, F. : "Note pour servir à l'étude des concrétions muqueuses membraniformes de l'intestin." Union méd., Nos. 7-9, 1869.

${ }^{5}$ Whitehead, W. : "Mlucous Disease." British Medical Journal, February 11, 1871, p. 140. 
The diet is perhaps the point of all others where the greatest mistake is made. An idea, strongly felt by the patient, that a great amount of strengthening food is required, leads to the further exhaustion of an already enfeebled digestion. Impress upon the patients the fact that it is the quantity absorbed which means streugth, and not the bulk swallowed, and it is possible to check the error they are so anxious to commit. Certain articles of diet should be strictly interdicted, the chief of which are the following: Liquid food, excepting milk, aggravates in the majority of cases every symptom; sugar is invariably hurtful; tea, coffee, and alcohol-Burgundy being the only wine from which I have ever derived benefit-regetables, and fruit also prove injurious."

Cruveilhier ${ }^{1}$ and Laboulbène ${ }^{2}$ discuss this ailment under the term "pseudo-membranous euteritis."

One of the best papers upon this disease was written by Da Costa, ${ }^{3}$ who called it "membranous enteritis." This author gave a full description of this affection, recognized its nervous character, furnished several detailed cases, and put particular stress upon dietetic treatment. 'Da Costa permits eggs, milk, bread, and solid food, which is better borne than liquids; tea, coffee, and alcoholic stimulants are to be permitted only in rery small quantities. As regards vegetables, we must observe whether they pass unchanged in the stools. Fresh meat juice is serviceable; from an exclusive milk diet, even faithfully carried ont, he has seen no good. Furthermore, Da Costa recommends that great attention be paid to the action of the skin, and

1 Cruveilhier: Anat. path. gén., t. ii.

2 Laboulbène: "Recherches sur les affections pseudomembraneuses," 1861.

3 J. M. Da Costa: "Membranous Enteritis." American Journal of the Medical Sciences, 1871, p. 321 . 
believes baths followed by systematic friction to be very useful. Daily moderate exercise is advocated, particularly in cool weather, and if possible an occasional trip to the mountains and living out of doors in the bracing mountain air. Everything that can be done to invigorate the digestive and nervous systems forms the essential part of the therapeutics.

A few years later there appeared an article by Edwards, ${ }^{2}$ who coincided with $\mathrm{Da}$ Costa's views in most points, being, however, much stricter with regard to diet. $\mathrm{He}$ says: "Easily digested or" even predigested food should be supplied, and care should be taken that undigested particles of food are not irritating the intestinal canal."

Leyden, ${ }^{2}$ in 1882, directed attention to membranous enteritis in Germany, where also very soon appeared exhaustive publications on this subject. Nothnagel ${ }^{3}$ suggested the name "colica mucosa," in order to show that a true enteritis need not exist in these cases and that the disease really is a mucous colic. Rothmann " was the first to publish a case of membranous enteritis-complicated with cancer of the skull-in which an antopsy was made. By means of Weigert's stain, or rather by Ehrlich-Hoyer's thionin (a specific stain for mucus), double-stained specimens could be obtained, which showed the presence of large quantities of mucus on the surface of the large bowel in the glandular tubules.

'Edwards : American Journal of the Medical Sciences, April, 1888, p. 329.

2 E. Leyden : Verhandl. d. Vereins f. innere Medicin in Berlin, Deutsche med. Wochenschr., 1882, Nos. 16 and 17.

${ }^{3}$ Notbnagel: "Colica mucosa." Beiträge zur Physiologie und Pathologie des Darms," 12tes Capitel, 1884.

4 Max Rothmann: "Ueber Enteritis membranacea." Deutsche med. Wochenschr., 1893, p. 999. 
Ewald, ${ }^{1}$ Boas, ${ }^{2}$ Kittagawa, ${ }^{3}$ Pariser, ${ }^{4}$ and others have added further contributions.

Ewald laid stress on a ptosis of the colon, Boas on atony of this organ as important factors in this affection.

Etiology. - Most authors agree that membranous enteritis is quite a rare affection; it occurs muclr more frequentiy in women than in men (children being only exceptionally affected).

That the nervous element (hysteria, neurasthenia) plays a great rôle in the origin of this trouble, no one can doubt, and W. Mendelson ${ }^{5}$ is right when he asserts that neurasthenia is not absent in any of his cases. Mendelson goes too far, however, when he says: "I believe that the reverse of the proposition may also as confidently be affirmednamely, that if neurasthenic patients be closely questioned, very few will be found who have not had at some time repeated characteristic passages of stringy mucus, associated with abdominal paius." Membranous enteritis is found in nervous individuals (possibly the affection as such adds much to their neurasthenia); but only a small fraction of the great mass of neurasthenics is afflicted with this ailment.

With regard to the frequency of membranous enteritis, $I$ examined my private patients of the year 1897 relative to its presence, and take the following data from my daybook. The total number of patients was 1,315-772 men, 543 women. Twenty of these patients suffered from mem-

1 C. A. Ewald: "Membranous or Mucous Enteritis." Twentieth Century Practice of Medicine, vol. ix., p. 265.

2 J. Boas : Deutsche med. Wochenschr., 1893, No. 41.

${ }^{3}$ O. Kittagawa: "Beiträge zur Kenntniss der Enteritis membranacea." Zeitschr. f. klin. Medicin, 1891.

4 Pariser: Deutsche med. Wochenschr., 1893, No. 41.

"Walter Alendelson: "Mucous Colitis a Functional Neurosis." Medical Record, January 30, 1897. 
branous enteritis-two men and eighteen women. The frequency of membranous enteritis among sufferers from digestive disorders expressed in percentages is, in men, 0.25 per cent; and in women, 3.31 per cent. Among these twenty patients, twelve had enteroptosis in a pronounced degree. Ewald has already pointed out that a prolapse of the colon is frequently found in patients with membranons enteritis. My own observations fully confirm this statement, for with the prolapse of the stomach descent of the colon naturally must be presupposed. It appears that enteroptosis certainly creates a fruitful soil for the development of membranous enteritis, although it does not directly cause it. Enteroptosis is, as is well known, very frecunent, while membranous enteritis is rare in comparison with the former. There must, therefore, be still other factors which are of importance in the causation of membranons enteritis.

With reference to gastric secretion and the motor function of the stomach in this disease, $I^{1}$ hare made examinations on twelve cases and found the following two points most conspicuous:

1. The motor function (prochoresis) of the stomachjudged from the amount of contents found one hour after the test breakfast-was increased in eight cases and nor$\mathrm{mal}$ in the four remaining.

2. Five cases presented a ty pical achylia gastrica.

Considering the comparative infrequency of achylia gastrica, which hardly amounts to two or three per cent of the digestive disorders, this large proportion of achylia in patients with membranous enteritis-namely, five in twelveis certainly noteworthy.

'Max Einhorn : "Membranous Enteritis." Medical Record, January $28,1899$. 
Three cases of membranous enteritis with normal acidity revealed, besides the increased prochoresis, still another feature in common with achylia-namely, the extraordinarily small amount of fluid surrounding the scarcely changed particles of roll, one hour after the test breakfast. Although this symptom may occasionally be met with in other cases than acliylia, it is nevertheless, as a whole, characteristic of this affection. Therefore we are justified in making the following statement: In many cases of membranous enteritis typical achylia is present, in some it is lacking, but even then some features characteristic to achylia are encountered. In membranous enteritis achylia thus plays a great part. Whether one condition causes the other, or one and the same factor (nervous influences) creates both, is difficult to say. The latter, however, is more plausible.

Symptomatology. - The disease is characterized by attacks of rather violent colicky pains in the abdomen, which are followed by the passage of mucous masses with the stools. The mucus may be voided either alone, without any admixture of fecal matter, or it forms a considerable part of the evacuation. Usually the attack is preceded by a period of obstinate constipation, and often followed by diarrhœa lasting a few days, and sometimes accompanied by tenesmus. Gastric symptoms-as loss of appetite, frequent belching, now and again a burning sensation at the pit of the stomach-are generally quite pronounced during the attack. Vomiting may occasionally appear, while fever is, as a rule, absent. The attack lasts three to seven days, and then the pains subside, the diarrhoa ceases, and euphoria reappears. More or less constipation, however, and some other dyspeptic as well as nervous symptoms persist. These free intervals last various periods of time 
(four weeks to five or six months). In rare instances the mucous discharges may be present continuously.

With reference to the mucous masses, they present a grayish-white appearance, seldom yellowish, and have either a ribbon-like or membranous form; at times the pieces are several feet long; ordinarily, however, they are cousiderably smaller. Complete moulds of the intestinal lumen have been observed by several authors, and Leyden not unjustly has compared this process with that of croup of the larynx. As already stated by Cornil,' the false membranes consist of mucus, mixed with dried-up epithelial oroid cells, which arise from a mucous metamorphosis of the cylindrical cells or the leucocytes. Nothnagel and others have proven the mucous nature of these discharges.

As suggested by Pariser, the mucous nature of these masses can be demonstrated by treating them, first, with sublimate alcohol, and then staining them with Ehrlich's triacid solution. A green color appears, which indicates mucus (fibrin treated in the same manner assumes a red color). Judging from my experience it is unnecessary to dip these membranes first into sublimate alcohol, as the same result will follow when they are put directly into the weak triacid solution. Microscopically this substance reveals a somewhat fibrillary nature, and contains many shrivelled cells, so called by Nothnagel. Micro-organisms are found admixed, although they do not seem to play any important part in this affection. In two of my cases microscopically single-celled corpuscles were found in these masses, having a distinct nucleus and a tail-like process. The accompanying drawing shows these corpuscles (Fig. 38). These are most probably metamorphosed goblet cells.

1 Cornil : Cited from Siredey. See above. 
Diagnosis.-The diagnosis of membranous enteritis is, as a whole, simple when the above-mentioned characteristic symptoms, including the mucous discharges, are present. It is, howerer, necessary to be careful not to mistake for mucus other substances admixed in the fæces,

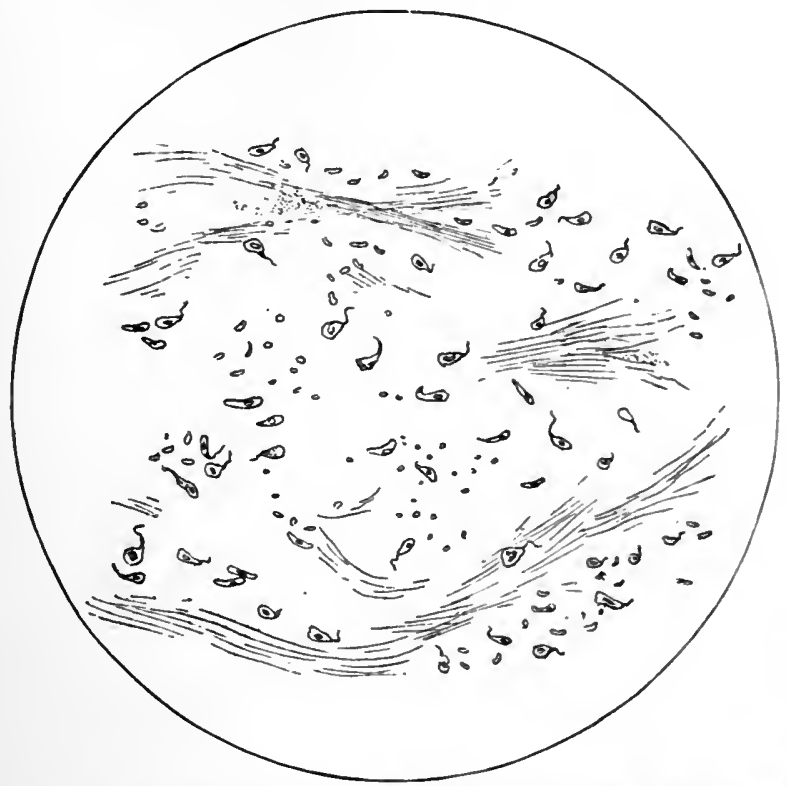

Fre. 38.-Microscopical Picture of Mucous Masses Found in the Evacuation of Mrs. L., Showing Numerous Cells Having a Nucleus and a Tall-like Process.

which occasionally resemble shreds of mucous membrane -as, for instance, the fibre of an orange, tendons, pieces of tapeworm. A microscopical examination will guard against all such errors.

This affection will hardly be confounded with real intestinal catarrh, as it presents an entirely different picture and only occasionally may have an abundant secretion of mucus in common with mucous colic. There are, howerer, 
cases of chronic intestinal catarrh which are complicated with membranous enteritis-that is, having typical attacks of mucous colic. The following case presents an instance of this kind:

Miss L. N-C, twenty-eight years old, had diarrhœa eleven years ago for quite a while, which disappeared after two or three months. The patient was then well until four years ago, when she again began to be troubled with diarrhoea. Soon periods of obstinate constipation appeared, which alternated with diarrhoea. The patient reports having occasionally observed mucus in the passages; at times (about every five or six weeks) there appear abdominal paius for about one or two hours, followed by an evacuation of pure mucus, the quantity being one to two tablespoonfuls. The appetite was always good. Now and again there was belching. The patient lost about twentyfive pounds in weight. Sleep is undisturbed, only at times restless for a few days. Her strength greatly failed. Palpation of the abdomen reveals spots sensitive to pressure in the entire course of the colon. The examination of the freces in the free interval shows small quantities of mucus well mixed with the fecal matter. The mucous masses voided after an attack of pains are free from fecal matter, appearing grayish-white and staining green when treated with Ehrlich's triacid solution.

Treatment.-Diet plays the principal part in the treatment of membranous enteritis. While the older writers laid stress on scanty light food, it is now generally accepted that abundant nutrition is of the greatest value. That a fluid diet is unsuitable, the older authors have already been cognizant of ( $\mathrm{Da}$ Costa, Whitehead, Siredey), and this axiom holds good in its entirety even to-day.

Recently von Noorden ' advised a very coarse diet, being

${ }^{1}$ C. von Noorden: "Ueber die Behandlung der Colica mucosa." Zeitschr. f. practische Aerzte, 1898, No. 1. 
guided by the idea that the intestinal tract should be exercised and strengthened by increased work. $\mathrm{He}$ recommends per day half a pound of bread containing plenty of chaff, leguminous vegetables, garden vegetables rich in cellulose, fruits with small pits and coarse skin, as currants, gooseberries, grapes-these being foods rich in undigestible material, thus forming much ballast for the bowel. Among fifteen patients subjected to this treatment by ron Noorden, seven were permanently cured, seven improved, and one was unchanged.

This method has certainly much in its favor; it may be better, however, not to institute this diet abruptly, as suggested by vou Noorden, but rather gradually.

I, for my part, for some years past have seen to it that my patients partook of an abundant and nutritious diet, without, however, advising substances that were too coarse. As a whole, I recommend ample food and try to keep the patients on a mixed diet containing plenty of regetables. In patients who have lived on a strict diet (as for instance milk diet or beef and hot water), I arrange the change gradually. The principle here is the same as stated by von Noorden, only not carried to such an extreme. It appears sufficient if the intestines of the patient with membranous enteritis are trained to master the foods customary in healthy persons, and the accomplishment of this object is all that is required. If we subsequently see that the organism amply fulfils its work, a few less digestible foods may then be added. It is not necessary to recommend these immediately from the start, nor are they important for the cure.

With regard to therapeusis, two phases will have to be considered-the treatment during the attack and the treatment during the interval. In severe attacks, rest in bed, 
warm poultices over the abdomen, a cleansing enema (of ordinary warm water with the addition of some common table salt or essence of peppermint-one teaspoonful to a quart), and afterward the administration of codeine or opium, with or without belladonna, are of value. As long as the pains last it is necessary to give light food (small quantities frequently). In mild attacks a stay abed may not be requisite, nor the administration of an analgesic remedy, and the diet may be the same as during the interval.

In the interval free from pains the treatment consists in a methodical application of olive-oil enemas, as suggested by Kussmaul and Fleiner. ${ }^{1}$ These enemas are injected into the bowel at night, at blood temperature, the quantity being two hundred and fifty to five hundred cubic centimetres. The patient is then instructed to try and retain the oil in the bowel during the night. The patients seldom assert that they are disturbed in their sleep by these injections and have to answer nature's call. In such an instance the quantity of oil may be reduced to one hundred and fifty or one hundred cubic centimetres. The oil should be injected every night for three weeks; then every other night for three weeks, and twice weekly for four weeks; finally, once weekly for five or six months. Besides, patients must accustom themselves to a regular morning evacuation, by promptly visiting the closet every day at the same hour in the morning.

Next to abundant nourishment the methodical oil cure is of the greatest importance in the treatment of this affection, and the results achieved are, according to $\mathrm{my}$ experience, very satisfactory. The administration of oil injections in membranous enteritis is mentioned here and

${ }^{1}$ Fleiner. Berliner klin. Wochenschr., 1893, No. 3. 
there in recent literature, especially by Ewald, but its value must be placed much higher than heretofore. The oil has not only a favorable influence upon the constipation which is always present in this malady, but at the same time also effects a diminution or a disappearance of the mucous discharges. How the oil brings this about is difficult to say. The favorable effect may perhaps be explained by the circumstance that by means of the oil the intestine is not left in an empty condition during the night, and thereby a spasmodic contraction is aroided, which must be regarded as one of the principal factors in the formation of mucus.

It is evident, according to my statement with regard to the etiology, that enteroptosis and anomalies of the gastric functions (principally achylia) exist in a large number of these cases. It will, therefore, be necessary to bear these points in mind and to treat the cases accordingly. The neurotic symptoms present in these cases should not be neglected in the general plan of treatment. We shall have to pay attention to a regular hygienic mode of living and ample physical exercise. In suitable cases occasional hydrotherapeutic measures will be of value. The tonic remedies, like iron, arsenic, etc., will also prove beneficial.

\section{Intestinal Neurasthenia.}

Tho various intestinal neuroses hare been separately described. In practice combinations of different neuroses frequently occur. Following Rosenheim we designate such cases as intestinal neurasthenia. The appetite as a rule is good and the symptoms usually appear during the period when intestinal digestion takes place. The symptoms generally develop one to three hours after meals and consist in a feeling of pressure, tension, and sometimes of griping 
in the abdomen. Occasionally there may be a sensation of nausea, at times an evacuation of the bowels accompanied with painful sensations in the abdomen and in the anus. Sometimes palpitation of the heart occurs, sometimes again a sensation of flashes of heat or of cold extending upward. As a rule, the patients feel worse when resting, especially in the recumbent position, than when walking about. After a period of one or two hours the symptoms usually disappean, to return again later on after a meal.

Constipation is as a rule associated with this condition. The quality of the food does not seem to exert much influence upon the symptoms, although the latter are more marked after heavier meals. In a few instances, especially when the pains play a predominant part and borborygmi occur, diarrhœa is encountered. In these cases the diarrhoea appears in the middle of the night or toward early morning, and disturbs the patient's sleep. It is of diagnostic importance that the pains do not in any way depend upon the quality of the food. Indigestible foods, even taken in considerable quantity, are occasionally well borne, while at other times a small meal, consisting of the lightest food, causes severe symptoms: Intestinal neurasthenia is sometimes associated with gastric neurasthenia and completes the picture of the other.

In making the diagnosis of intestinal neurasthenia anatomical lesions of the intestines must first be excluded.

The treatment consists in hygienic measures which serve to tone up the system, in ample feeding, and in the administration of the bromides, occasionally in conjunction with iron and arsenic. With regard to diet all foods are allowed excepting indigestible substances, and a preponderance of vegetable food is to be recommended. 


\section{CHAPTER XII. \\ INTESTINAL PARASITES.}

General Remarks.-Most of the animal parasites found in man iuhabit the intestinal canal. Leuckart ' estimates the number of varieties at about fifty. Not all parasites, however, produce morbid conditions. Comparatively few of them evoke a pathological state, either in the intestine by their direct presence, or in the blood by the formation of toxic products which are absorbed and reach the circulation. The intestinal parasites are detected by repeatedly examining the stools. They may be seen or their presence may be assumed from the discovery of their ova (the latter referring to the helminths). There are no characteristic symptoms which would be encountered only in morbid conditions due to animal parasites. The diagnosis, therefore, must be made by directly discovering them or their eggs in the dejecta. It will always be wise to look for worms in cases in which gastric and intestinal symptoms of a functional character exist, accompanied or not by anæmia and certain neuropathic affections. The intestinal parasites are divided into two large groups: (1) Protozoa. (2) Vermes.

\section{PROTOZOA.}

Amober.

Besides dysenteric amobre which have been described above, a similar variety is occasionally encountered giving

${ }^{1}$ Leuckart: "Die menschlichen Parasiten," Leiprig, 1886, Bd. ii. 
rise to no symptoms whatever or sometimes to slight attacks of diarrhœea.

\section{Sporozoa.}

Among the sporozoa coccidia are occasionally found in the stools. This organism is egg-shaped, provided with a thin shell, $0.02 \mathrm{~mm}$. long, and contains in its interior a large number of nuclei usually arranged in groups. The coccidia do not seem to have any pathological bearing.

\section{Infusoria.}

To these belong cercomonas intestinalis, trichomonas intestinalis, and paramæcium coli. All of them are found principally in conditions in which diarrhœe is the foremost syimptom.

The cercomonas intestinalis is pear-shaped, has a distinct nucleus and eight flagellæ. The head portion of the body tapers obliquely and presents a depression (Fig. 39). It is not believed to have a direct pathogenic significance.
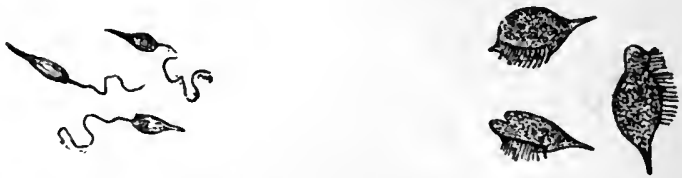

Fig. 39.-Cercomonas Intestinalis (Da- FIG. 40.-Trichomonas Intestinalis (Zunvaine). ker).

It is assumed, however, that this micro-organism is liable to prolong pre-existing catarrhal affections of the intestine.

Tirchomonas intestinalis presents the same features as the cercomonas and can be distinguished from the latter by its somewhat greater size and the row of fine cilia upon the periphery of its body (Fig. 40). In fresh dejecta this micro-organism moves around very actively. Zunker ${ }^{1}$ found

IZunker: Deutsche Zeitschr. f praktische Medicin, 1878, No. 1. 
it principally in mushy dejecta having a brownish-yellow color and a somewhat putrid odor.

Paramcecium (or balantidium) coli is egr-shaped, $0.1 \mathrm{~mm}$. long and covered with fine cilia, the latter being densely grouped about the mouth, while but few of them surround the anus. In the interior of this organism are found a nucleus and two contractible vesicles, besides fat droplets, starchy particles, etc. (Fig. 41). The balantidium coli was first described by Malmsten' in 1857. In the fresh stools the balantidium moves about very rapidly, but it dies as early as one-half an hour to two hours after the dejecta have been passed. Like the cercomonas, the paramrecium coli is believed to keep up conditions of diarrhœa.

The treatment directed against these infusoria consists in intestinal irriga-

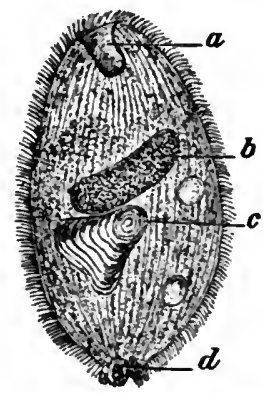

F1G. 41. - Balantidium Coli (Claus). a. Mouth: $b$, nucleus: $c$, a granule of starch which has beell ingested ; $d$, a foreign body in the process of being expelled. Highly magnifled. tion with watery solutions of tannic acid, boracic acid, thymol, or quinine.

\section{VERMES.}

\section{Cestodes (Tape Worms).}

General Remarks.-In describing the disorders caused by tapeworms it is best to include the tænia solium, tænia mediocanellata, and bothriocephalus latus.

The symptoms produced by these three different entozoa are almost identical. In some instances the tapeworm is domiciled in the intestine for a long period of time without manifesting any symptoms. The host may enjoy per-

'Malmsten: Virchow's Archiv, Bd. xii. 
fect liealth and only after noticing segments of tronia in the dejecta does he become conscious of his uninvited guest. In other instanices the worm produces intestinal as well as general disturbances. A feeling of pressure at the pit of the stomach and pains at different points of the abdomen may be present. Bulimia is frequently encountered. Anorexia and anorexia alternating with bulimia are also occasionally observed. Nausea, even romiting, may be present, especially in the morning. The bowels are usually constipated. In a few instances, however, there is persistent diarrhoea.

Besides these gastro-intestinal symptoms there may be present various disturbances of the nerrons system or of the blood; dizziness, headache, fainting spells, convulsions, epilepsy, various forms of paræsthesia of the extremities. Some patients, again, look very bad and become emaciated, notwithstanding that they take sufficient quantities of food. The anæmic condition is occasionally very marked. The patient feels extremely weak, suffers from palpitation of the heart, is hardly able to walk, and is subject to fainting spells. In this serious form of aniemia oedema of the feet and eyelids may exist as well as hemorrhages from the mucous membranes. The microscopical examination of the blood in these instances reveals poikilocytosis and also nucleated red blood corpuscles, thus demonstrating the existence of a progressive pernicious anæmia. The grave condition just described has been observed only in the presence of bothriocephalus latus but not of the other varieties of tapeworms.

The proof that the symptoms described are produced by the tapeworm is found in the circumstance that they disappear entirely after the remoral of the parasite.

None of the abcre symptoms, however, permits the diag- 
nosis of tapeworm, for they are found also when it is not present. The diagnosis can be made only by the discovery of either segments of the parasite or their eggs in the stools.

The tapeworm has a head or scolex, which may remain alive for years, - eren when separated from the other part of the body,-an obloug neck and detachable segments (proglottides). The latter vary in size and in configuration the farther away from the head they are situated. They possess the power of moving. The tapeworm is a flat worm devoid of mouth or intestine. It grows by alternate generation through the germination of a pear-shaped primary host (head) and remains united with the latter for a considerable time as a long band-shaped colony. Each member of the colony forms a sexually actire individual. The proglottides increase in size the more distant they are from the head. The tapeworm is an hermaphrodite. It is provided on its head with four sucking discs, by means of which it is enabled to attach itself to the intestinal mucosa. It derives its nourishment by means of pores from the intestinal chyne. The older proglottides contaiu a large number of fructified eggs. The latter are off and on emptied into the intestinal canal and then appear in the dejecta.

The ovum contains an embryo which requires for its development an intermediary host. After reaching the stomach of the intermediary host the envelope of the ovum is dissolved by the gastric juice. The embryo is now set free and finds its way either by the lymphatics or by the blood-ressels to some place (usually the muscles) where it settles. Here it surrounds itself with a sac, which later on may become surrounded with a calcareous deposit. In this condition the embryo is called cysticercus or measle. 
When the measle again reaches the stomach of a new host it then opens and its scolex advances into the small intestine, where it develops into a full-grown tænia.

Teria Solium.-Tænia solium, or the armed tapeworm, when fully developed, is from two to three metres long. Its head is of pinhead size and spherical in shape. It has four cuplike suckers, in the middle of which is situated the rostellum, the latter being surrounded with a large number of hooks (Fig. 42). These are arranged in two rows and number from twenty-four to twenty-six. Succeeding the head is a filiform neck, almost an inch long. Commencing

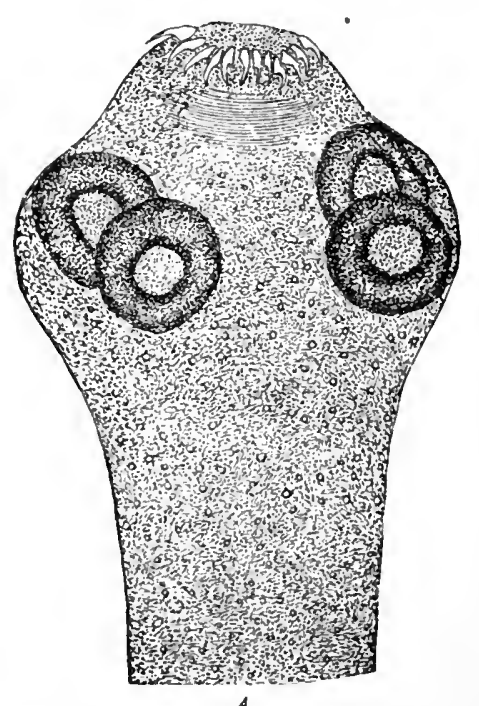

A.

Fig. 42.-Head of Tænia Solium with Protruding Rostellum. Magnifled 50 diameters. (Zlegier.) at a certain distance from the head the body is divided into segments. The mature proglottides

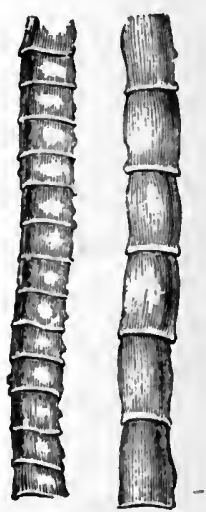

Fig. 43.-Half Developed and Fully Matured Segments. Natural size. (Leuckart.)

are 1 to $1.5 \mathrm{~cm}$. long and $6 \mathrm{~mm}$. wide. The genital opening is situated at the side near the posterior border of the segment (Fig. 43). The nterus forms a straight median tube, giving off at right angles five to seven branches on 
each side. These branches are undivided at first, but toward the periphery ramify in the form of a tuft (Fig. 44). The eggs are round and provided with a thick shell.

Tænia solium inhabits the small intestine of human beings. The further development of the embryo into

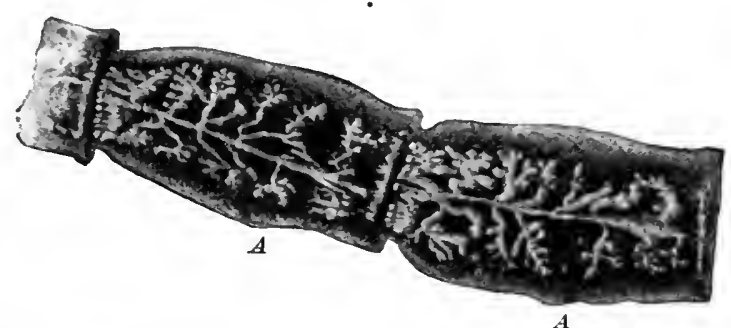

FIG. 4L-Tænia Solium. Showing two proglottides. $A, A$, pores. (Huber.)

measles occurs in the intermediary host, the pig, in which condition they reach the human system and are transformed into mature trenias. Rarely the measles (cystocercus cellulosa) are found in men, in which instance they occur in various organs, brain, eye, skin, etc. The gravity of the disease which they produce depends upon the importance of the organ they involve.

Tronia Saginata or Mediocanellata.-This tapeworm is the one most frequently observed in America as well as abroad. The tænia saginata is much longer, thicker, and wider than tænia solium. The head is $2.5 \mathrm{~mm}$. large, has four large sucking-dises but no rostellum, and is often pigmented (Fig. 45). The length of the worm is 4 to 5 metres, the proglottides are unusually thick, the widest being in the middle. The mature segments occasionally attain a length of $2.5 \mathrm{~cm}$. The uterus lies in the middle of the segment and gives off numerous brauches on both sides (about twenty on each side (Fig. 46). The genital opening is situated on the side below the middle. The 
eggs have an elliptical shape, a brownish color, and a contour exhibiting radiating streaks.

The trnia saginata inhabits the small intestine of man. Its measles occur in beef, as has been demonstrated by

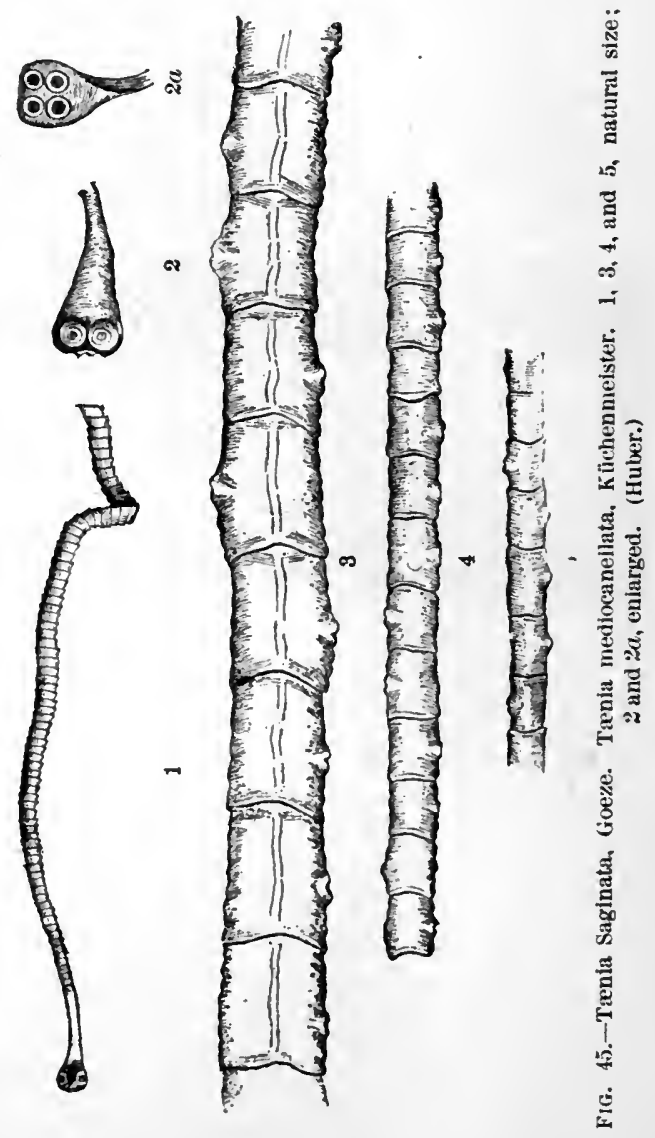

Huber ${ }^{1}$ and Leuckart. These measles are usually smaller than those of traia solium. Human beings acquire this

${ }^{1}$ Huber: "Twentieth Century Practice of Medicine," vol. viii., p. $5 \% 0$. 
tænia by the consumption of raw beef. The measles have not as yet been found in man.

- Bothriocephalus Latus, Tcenia Lata or Pig Head.-This tapeworm is the longest. It measures from fire to eight metres. The head is elongated, of almond shape, being about $2.5 \mathrm{~mm}$. in length (Fig. 47). It has two lengthy big grooves on its flat surface (Fig. 48). The neck is narrow, about $2 \mathrm{~cm}$. long. The body is thin and flat like a ribbon, excepting the central part of the segments which

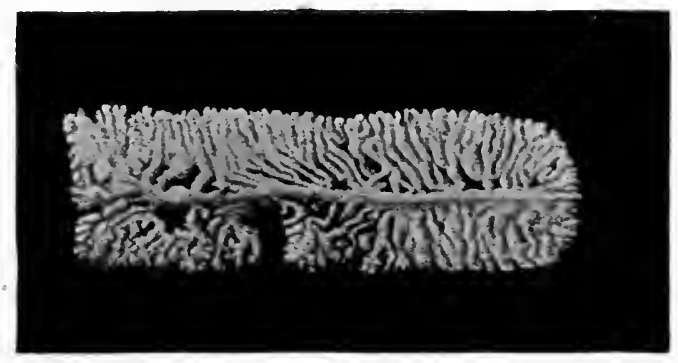

Fig. 46.-The Uterus and its Branches in a segment of Tænia Saginata. Enlarged 3 diameters. (Huber.)

project somewhat outward. The genital openings are on the flat surface in the middle, the female very close to the male. The uterus has a special opening and four to six visible uterine convolutions on each side, which look almost like a rosette. The eggs are oval, round, with a thin membraue and a lid (Fig. 49). 'They measure $0.07 \mathrm{~mm}$. in length and $0.04 \mathrm{in}$ width.

The measle of bothriocephalus latus occurs principally in fish, especially in pike, turbot, perch, and tront.

The trenia lata lives in the small intestine of man, but is also, though rarely, found in dogs. In the northeastern part of Europe, Holland, Switzerland, and Japan this tapeworm is very prevalent. In America it occurs but in- 
frequently. As stated above, among the symptoms produced by bothriocephalus anæmia is often observed.

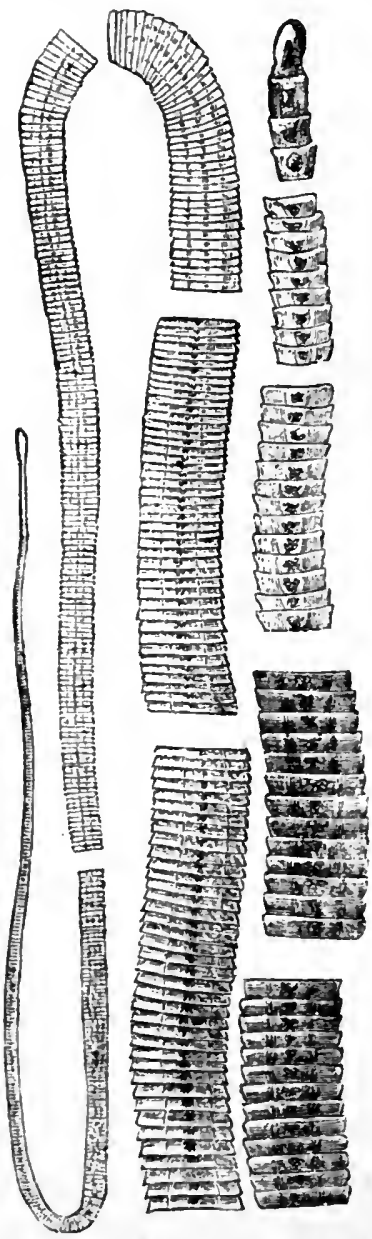

FIG. 47.-Bothriocephalus Latus. Natural size. (Leuckart.)

Aside from the three tapeworms just described there exist a few more varieties which are only rarely met in human beings. They are:

(1) Tenia Nana. - This is the smallest tapeworm found in man. It measures 10 to $15 \mathrm{~mm}$. in length and may have one hundred and ninety segments. The head has four sucking-discs, a rostellum, twenty-four to twenty-eight hooklets in a single row. The proglottides are short and broad; the genital openiugs are on one side. This tapeworm has been observed principally in Egypt and Italy in children. It usually occurs in large numbers in the small intestine, from forty to even five thousand. The symptoms produced by this tapeworm are mostly nervous disturbances, fainting spells, occasionally eren epilepsy.

(2) Tenia Cucumerina.This small cucumber-shaped tapeworm occurs frequently in the intestine of the dog, but has also been found, although rarely, in small children. The tapeworm is 10 to $40 \mathrm{~cm}$. 
long and about $3 \mathrm{~mm}$. wide. The measle of this tænia inhabits the flea.

(3) Ternia Flavo-Punctata or Toric Dimimeta.-This parasite is 2 to $6 \mathrm{~cm}$. long and $3.5 \mathrm{~mm}$. wide. Its head is very small, club-shaped, and provided with suckingdiscs. The measle infests the caterpillar and cocoon of

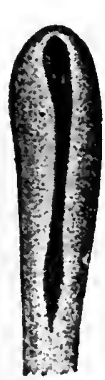

FIG. 48.

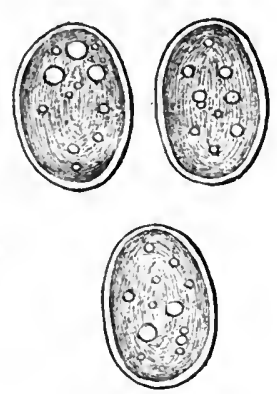

Fig. 49.

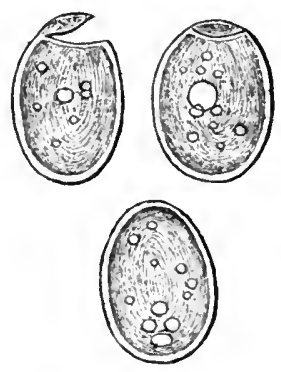

Fig. 48.-Head of Bothriocephalus Latus. Magnifled. (Heller.) Fig. 49.-Eggs of Botbriocephalus. (Krabbe.)

asopia famialis and in the coleoptera axispinosa. This tapeworm has been observed in man only a few times.

(4) Bothriocephalus Cordatus. - This tapeworm resembles in all particulars the bothriocephalus latus except that it is much shorter and that the head merges into the proglottides directly without an intermediary neck. It occurs in the intestine of men and dogs in Greenland.

The list of the tapeworms enumerated above is not complete, for there exist the trenia madagascariensis, bothriocephalus liguloides, and others, but as these do not occur in Europe or America a description of them does not appear to be of practical interest.

Treatment.-Prophylaxis. In order to escape infection with tapeworm it is necessary to abstain from raw or medium done meats, including fish. The sanitary inspection 
of the meat is no absolute guarantee that it is free of measles. Thorough boiling or broiling of the meat destroys the cysticerci and thus the danger is aroided. In order to diminish the spread of tapeworm it is advisable to free the patient of the worms and thoroughly to destroy them as soon as possible after they have left the intestine. Whoever examines the proglottides or the ova should carefully wash and disinfect his hands immediately afterward in order to avoid auto-infection.

The direct treatment of the tapeworm consists in measures to expel it from the intestinal canal. This is accomplished by emptying the bowels previously and giving a vermifuge afterward. The treatment is carried out in the following way: For about two days before giving the vermifuge the patient is kept on a scanty diet, consisting of some milk, meat and broth, very little bread or none at all. A laxative (calomel eight to ten grains or castor oil one tablespoonful) is given once a day. On the evening preceding the administration of the vermifuge the patient should have no supper or should take only salt herrings with onions. On the following morning a cup of coffee or tea is given. Half an hour to one hour later the vermifuge is administered. Among the drugs for the removal of the tapeworms the following are the most efficient:

Male-fern extract is given in doses of 6 to $10 \mathrm{gm}$. ( 3 iss.iiss.), as for instance:

R Extr. filicis mar. ather............... 8.0 ( 3 ij.)

Syr. simpl...................... $40.0\left(\frac{3}{3} i \frac{1}{8}\right)$

S. To be taken in ten minutes.

The dose of male-fern should nerer be very high and should not exceed $10 \mathrm{gm}$. ( 3 iiss.), as symptoms of intoxication have frequently been observed.

Pomegranate root is also an efficient remedy, espe- 
cially if it is fresh. It may be given in an infusion of the bark, three ounces of which are macerated in ten ounces of water and then reduced to one-half by evaporation. The entire quantity is then taken within half an hour.

Pelletierine, the active principle of pomegranate root, may also be used in doses of five to eight grains.

Flores koosso, about 20 to $30 \mathrm{gm}$. ( $z \mathrm{v}$. to $\xi i$.) of the blossoms are thoroughly mixed in sugar water or lemonade and should be taken within one-half or one hour, or-

R. Flores koosso,

Mellis despumati................. āā 3 ₹. (20 gm.)

Fiat electuarium. S. To be taken in two portions.

Kamala may also be employed in doses of $10 \mathrm{gm}$. ( 3 iiss.) mixed in aqua foeniculi or in wine and taken in the same way.

Turpentine 30 to $60 \mathrm{gm}$. ( $\bar{j}$ i.-ii.) may be given in capsules. After this medicament one or two glassfuls of milk should be taken.

Pumpkin seeds (semina cucurbitæ) may be administered in doses of $120 \mathrm{gm}$. ( $\overline{\mathrm{iv}}_{\mathrm{iv}}$ ), thoroughly mixed with the same amount of grape sugar.

Cocoanut has also been recommended for this purpose. The milk and albumin of an entire nut should be consumed within one hour.

Naphthalin in doses of 0.6 to $2.0 \mathrm{gm}$. (gr. $\mathrm{x} .-\mathrm{xxx}$.) may be given in capsules.

Salol 3 gm. (gr. xlv.) in eapsules may also be advantageously employed.

One or two hours after the administration of the vermifuge a cathartic should be given, usually about two tablespoonfuls of castor oil, or citrâte of magnesia one to two teaspoonfuls. The resulting evacuation must be thor- 
oughly examined and the tapeworm looked for, especially its head.

Children require a correspondingly smaller dose of the above remedies, according to their age. Patients who are debilitated, or have intestinal disorders or organic lesions of the digestive tract, should not be subjected to this treatment, nor should it be employed shortly after typhoid ferer or other grave diseases. In these conditions it is necessary to postpone the treatment until a more opportune time.

\section{Tiematodes (Fluke Worms).}

The trematodes are solid worms of a tongue or leaf shape. They possess a clinging apparatus in the form of

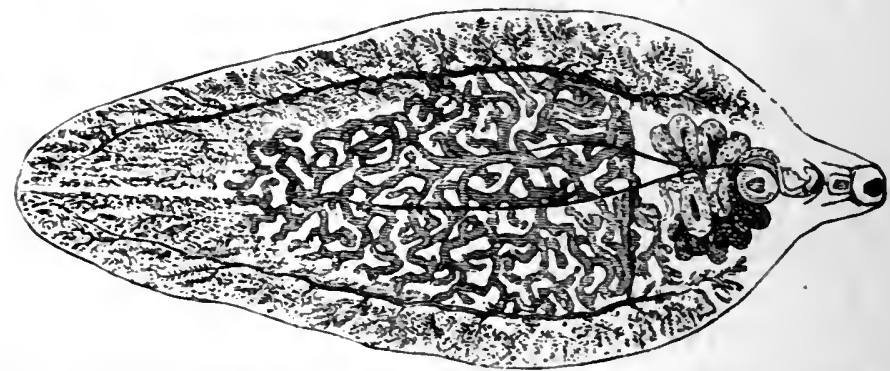

Fig. 50.-Distoma Hepaticum, with Male and Female Sexual Apparatus. (Leuckart.) Magnlleed 23/2 diameters.

oral and ventral sucking-cups varying in number. Sometimes they are also provided with hook or clasp like projections for this purpose. The intestinal canal is without any anus and is split like a fork nearly throughout its extent. The fluke worms are mostly hermaphroditic. To these belong:

Distoma Hepaticum or Liver Fluke.-This parasite has a leaf shape, is $22 \mathrm{~mm}$. long and $12 \mathrm{~mm}$. wide. The cephalic end projects like a beak and bears a small cuplike 
sucker, in which the mouth is located. Close behind this on the rentral surface is a second suction cup and between the two lies the sexual orifice. The uterus consists of a
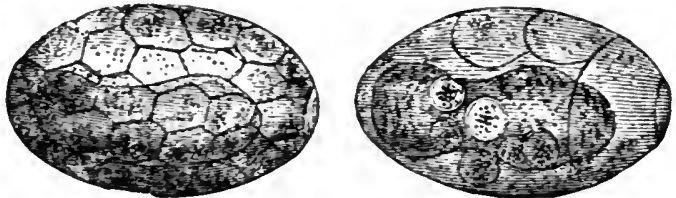

Fig. 51.-Eggs of Distorna Hepaticum. (Leuckart.) Magnifled 200 diameters.

conroluted bulb-shaned bag, situated behind the posterior sucker. On each side of the body lie the orisacs and between them the much branched testicular canals (see Fig. 50). The eggs are oval, $0.13 \mathrm{~mm}$. long and $0.08 \mathrm{~mm}$. wide. They have a brownish color and are prorided with a lid (Fig. 51).

The liver fluke is rare in man, though frequently found in ruminating animals. It inhabits the biliary ducts and is occasionally found in the intestine and in the inferior vena cara. The symptoms which it produces are raried: jaundice, enlargement of the liver, diarrhœea, hemorrhages.

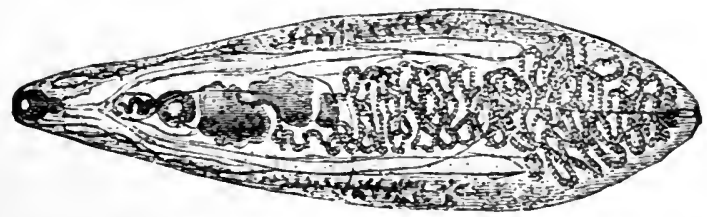

FIG. 52.-Distoma Lanceolatum with its Inner Organs. (Leuckart.) Yagnifled 10 diameters.

Most probably the liver fluke reaches the intestinal canal by means of impure water or regetables.

Distoma lanceolatum is 8 to $9 \mathrm{~mm}$. long and 2 to $2.5 \mathrm{~mm}$. wide. It has a lancet shape and the head portion is not specially marked off from the bodr (Fig. 52). The eggs 
are considerably smaller than those of distoma hepaticum, being only $0.04 \mathrm{~mm}$. long ( Fig. 53). With regard to its occurrence and symptoms it resembles the liver fluke.

Distoma hamatobium or Bilharzia hematobia is frequeutly found in hot climates, especially in Egypt. In
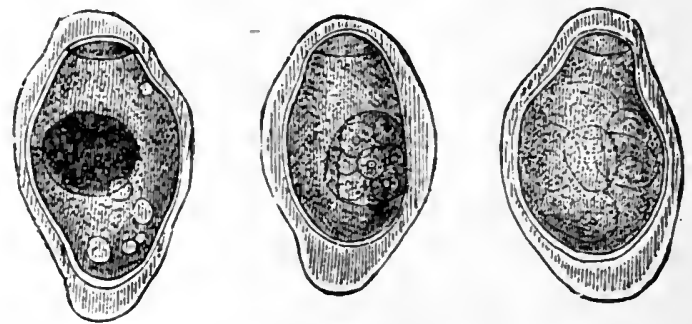

Fig. 53.-Egg of Distoma Lanceolatum Shortly After the Formation of a Shell. (Leuckart.) Sagnifled 400 diameters.

the United States and in Europe it is very rarely found. This parasite has separate sexes. The male is from 12 to $14 \mathrm{~mm}$. long. Its body is smooth, but in its posterior portion rolled up into a tube, which serves for the reception of the female (canalis gynæcophorus) (Figs. 54 and 55). The female is from 16 to $19 \mathrm{~mm}$. loug and almost

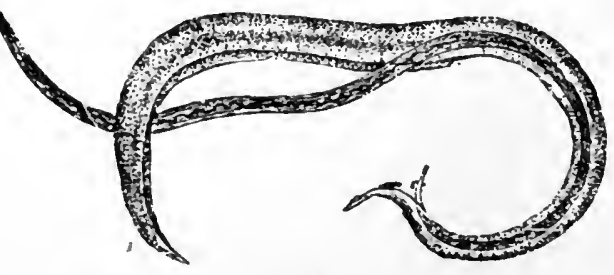

FIG. 54 .

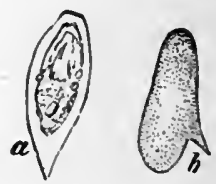

FI(1. 5 5.

Fig. 54.-Dlstoma Hæmatoblum. (Lenckart.) Nale and female, the latter in the canalls gynzecophorus of the former. Magnifled 10 diameters.

Fig. 53.-Eggs of Distoma Hæmatobium. (Leuckart.) $a$, Fgg with terminal spine; $b$, egg with lateral spine. Magnlfled 150 diameters.

cylindrical. The sexual opening lies in both sexes close behind the ventral sucker. The distoma hæmatobium 
- finds its way into the intestinal canal of man and then reaches the portal circulation, where it develops. In the intestinal canal it has been encountered very rarely, in which case ulcerations of the intestinal mucosa were present. It frequently causes hæmaturia and great cachexia, terminating fatally in some instances.

As regards treatment, the removal of these fluke worms must be undertaken in identically the same manner as that of the tapeworms described above.

\section{Nematodes (Round Worms).}

The round worms which occur as parasites have a slender, cylindrical, sometimes filiform body, with neither segments nor appendages. The integument is thick and elastic. The oral opening is at one extremity and provided with either soft or hornlike lips. The alimentary canal extends throughout the entire body cavity, terminating in an opening upon the ventral side at a short distance from the posterior extremity. The sexual organs and their orifices lie on the ventral surface. The female aperture is located at about the middle of the body; in the male the sexual orifice is situated close to the anus. The males are usually much smaller than the females.

Ascaris Lumbricoides (Common Spool or Round Worm).This worm is one of the most frequently observed parasites in man. The round worm has a light brown or reddish color and a cylindrical shape. The male is $20 \mathrm{~cm}$. and the female $30 \mathrm{~cm}$. long. The posterior extremity of the male is bent in the form of a hook and provided with two spicules or chitinous processes. The mouth is surrounded by three muscular lips provided with rery fine teeth. The sexual opening of the female lies anterior to the middle of the body (Fig. 56). The eggs when ripe 


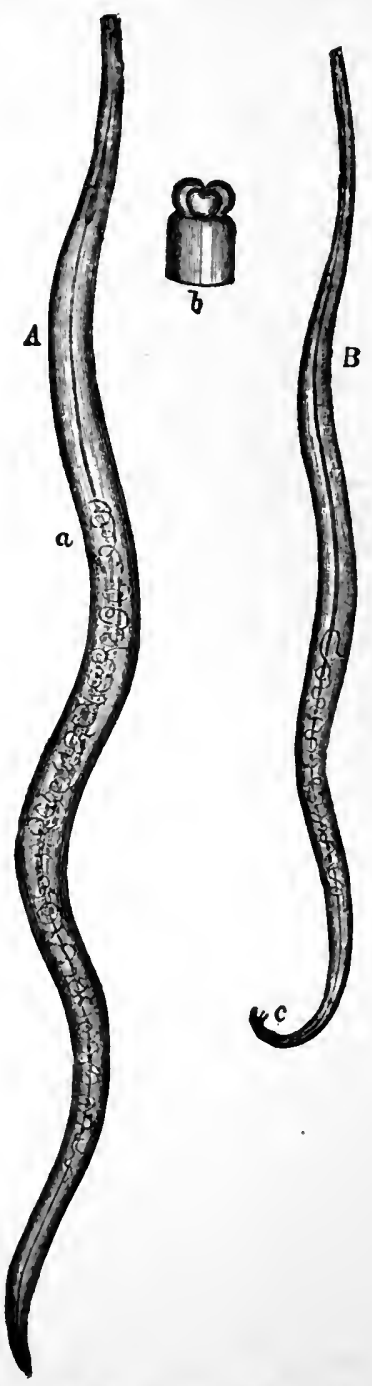

Fig. 56.-A scaris Lumbricoldes. (Perls.) $A$. Female: $\boldsymbol{B}$, male. (Natural size.) At $a$ is the female sexual orifle; $c$, the two spicules of the male: $b$, head extremity (magnifled) of the worm. with the three lips. have a double shell and around this is an albuminous envelope which is irregularly shaped, and studded with excrescences (Fig. 57). The long diameter of the egg is about $0.05 \mathrm{~mm}$.

The round worm possesses a strong odoriferous principle which is very perceptible eren after the worm has been carefully washed. According to Huber, ${ }^{1}$ this substance may occasion urticaria in persons predisposed to this eruption. It is not improbable that certain of the symptoms of ascariasis are due to the action of the same element.

The principal habitat of ascaris lumbricoides is the small intestiue of man. It develops here often in large numbers, fifty to one hundred and more occurring together. The mode of transmission, according to

${ }^{1}$ Huber: "Twentieth Century Practice of Medicine," vol. viii., p. 583. 
Leuckart, Grassi, ${ }^{2}$ and Lutz, ${ }^{2}$ is by ingestion of the eggs of the ascaris, there beiug no intermediate host. The full development of the round worm from the egg to its period of sexual maturity requires ten to twelve weeks. Infection usually takes place by eggs existing in the soil near dwelling-places, in the drinking-water, and also in some foods, principally salads and fruits. Ascaris lumbricoides is most frequently found in children three to twelve years old, the poorer classes showing a larger perceutage than the well-to-do. In grown persons the worm is not so frequent. The female sex is more frequently infected than the male.

The diagnosis of ascariasis is made

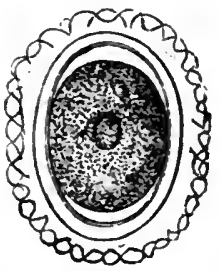

FiG. 5i.-Egg of Ascaris Lumbricoides (Leuckart) with shell and Albuminous Envelope. Magnifled 300 diameters. by the detection of the worm in the fecal matter, or of its eggs, which are easily recognized.

Symptoms.-Ascariasis may exist withont giving rise to any symptoms whaterer. Occasionally, however, there are various disturbances : anorexia, nausea, irregularity of the bowel, meteorism, an irregular pulse; in children black rings around the eyes, much nervousness, even convulsions. In rare instances progressire anæmia has been observed (Leichtenstern). Anatomically hyperæmia of the intestinal wall has been frequently found, erosious are rare. Itching of the nose is often present in ascariasis and may be due to the odoriferous principle.

The round-worm is liable to wander and may then give rise to severe complications. In several instances it has

${ }^{1}$ Grassi: Centralbl. f. Bacteriologie und Parasitenkunde, $188 \%$.

${ }^{2}$ Adolf Lutz: "Klinisches über Parasiten des Menschen und der Hausthiere." Centralbl. f. Bacteriologie, 1889. 
been found in the bile duct, in the gall bladder, and even in the liver, producing abscesses and even a fatal issue. The worm occasionally migrates into the stomach and produces pain and often vomiting. In the latter act it is often expelled from the mouth. Occasionally it ascends the osophagus and enters the larynx, causing asphyxia, and, in rare instances, even death. It has also been found in hernial sacs and in the peritoneal cavity, but it is generally believed that it cannot penetrate through the healthy intestinal wall. Obstruction of the bowels by a conglomeration of ascarides has also been thought possible; its real occurrence, however, is denied by Leichtenstern. ${ }^{1}$

Prophylaxis requires total destruction of all the eggs of the ascaris passed with the fecal matter of the patient. The grounds near dwellings should be kept perfectly clean and the hands should be frequently washed. All foods should be protected against a possible infection.

Treatment.-The treatment consists in freeing the patient from the worms. This is done in a similar manner as in the case of tapeworms. The intestinal tract is kept partially empty for a day or two before the administration of the anthelmintic. The most efficient remedy for this purpose is santonin, which is given in a dose of 0.02 to 0.06 gm. (gr. $\frac{1}{3}-\mathrm{i}$.) twice or four times a day. Then a purgative remedy is given. Some combine the santonin with the purgative and give them together. Thus santonin 0.2 (gr. iiiss.), castor oil $60 \mathrm{gm}$. ( $z^{\mathrm{ii}}$.), twice or three times daily one teaspoonful for small children, a dessertspoonful for larger children, and one tablespoonful for grown people.

'Leichtenstern: "Verengerungen, Verschliessungen und Lageverănderungen des Darms." von Ziemssen's Handibuch der spec. Path. und Therapie, Bal. vii., Abth. 2. 
The santonin may also be given in combination with calomel; thus-

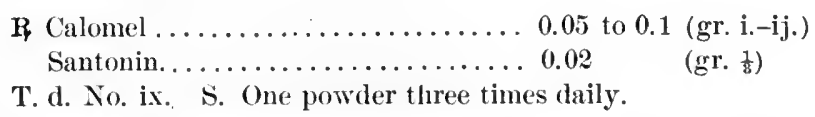

Flores cinæ, the plant from which santonin is obtained, may also be administered in doses of 0.5 to $2 \mathrm{gm}$. as powders or as an electuary, with the addition of jalap, 0.1 to $0.2 \mathrm{gm}$.

Chenopodium or wormseed is also a popular remedy, the powdered seeds being given in doses of 1 to $2 \mathrm{gm}$. (gr. $\mathrm{xv} .-\mathrm{xxx}$.), or the volatile oil in five to ten drop doses. Thymol has also been recommended in doses of 0.5 to $2 \mathrm{gm}$. (gr. vii.--xxx.) in twenty-four hours. It may be given in gelatin capsules. Irrigation of the bowels with water to which three to five drops of benzene have been added has likewise been suggested, but does not appear as beneficial as sautonin.

Ascaris Mystax. - A round-worm resembling ascaris lumbricoides but much smaller and somewhat thinner. This parasite frequently occurs in animals, principally in cats, but has been discovered very rarely in man. No symptoms whatever have been observed.

Oxyuris Vermicularis, Awltail, Seat or Pin Worm, Ilaggot or Thread Worm. - This parasite is white and filiform, 4 to $12 \mathrm{~mm}$. long and 0.2 to $0.6 \mathrm{~mm}$. thick (Fig. 58). The males are much smaller than the females. The oxyuris has three small knoblike lips. The female possesses two uteri passing backward and forward from the end of the vagina. The opening of the latter is situated above the middle of the body. The eggs are $0.05 \mathrm{~mm}$. long and 0.02 wide. The contents are granular and the shell appears white. 
Infection takes place when the eggs of oxyuris reach the stomach. Here the shell opens and the embryo migrates

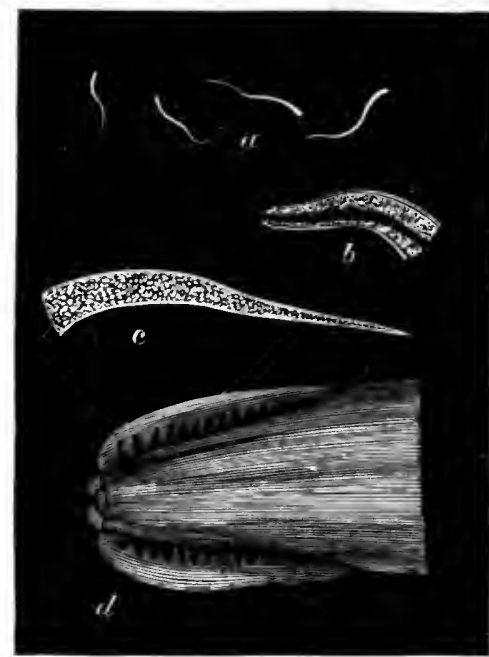

FIG. 58.-Oxyuris Vermicularis: $a$, natural size: $b$, head; $c$, taii, magnifled; $d$, head greatiy magnifled. into the small intestine (Fig. 59). After fructification has taken place the females usually begin to wander along the intestinal canal. 'In the cæcum they generally make quite a long sojourn until the eggs are almost ripe. Then they again begin to pass downward. According to Leichtenstern, Lutz, and Huber, the females do not pass their eggs within the intestinal canal. As a rule they first leave the bowel and then deposit the eggs. For this reason the fecal matter usually does not contain any eggs.

The symptoms which are most frequently observed consist in pronounced pruritus ani due to the irritation produced by the passing of the parasites out from the rectum. Frequently the itching annoys the patient as soon as he retires. Various nervous symptoms are occasionally observed: anorexia, nausea, dizziness, palpitation of the heart, pollutions and spermatorrhœea in the male; besides diarrhœa occasionally occurs. Pronounced anæemia is encountered, although rarely. In rare instances the parasites reach the vagina and cause irritation there. Nymphomania has then been observed.

Infection probably occurs through direct conveyance of 
the eggs by the unwashed hands of the host. It is also possible that ova dried by the sun exist on fruit, radishes, or salads, in which state they may be carried into the stomach.

The diagnosis of the thread worm is made by inspection of the anal region and by the finding of the oxyuris.

With regard to prophylaxis extreme cleanliness is of the greatest importance. Fruits should be thoroughly cleaned and then peeled before they are eaten. The eating utensils of a person infected with oxyuris should never be used by another, unless they have been thoroughly disinfected. The same applies to the clothes. Sleeping with an infected person should be forbidden, and even touching his hands
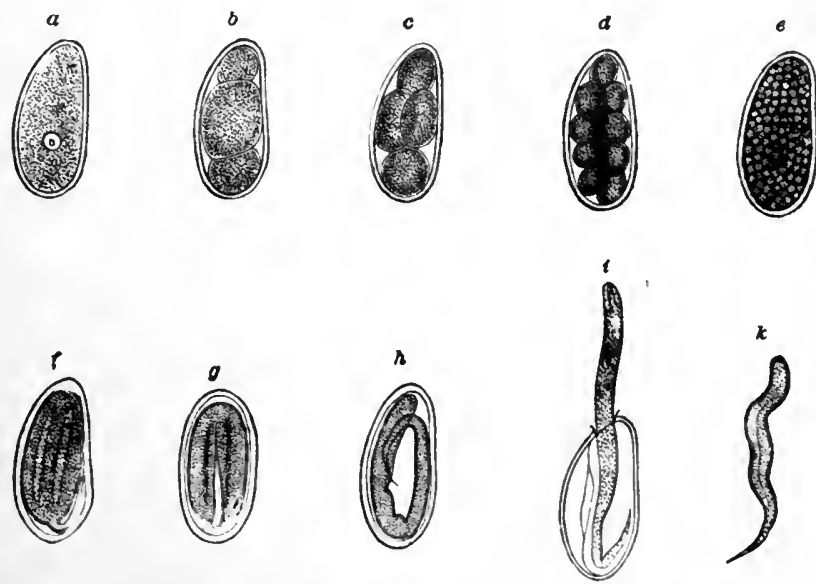

Fig. 59.-Deyclopment of Oxyuris Vermicularis. (Heller.) a-e, Segmentation of the yolk; $f$, ovum containing tadpole-shaped embryo, seen from the side: $g$, abdominal view of the same; $h$, ovum with worm-shaped embryo; $i$, embryo escaping from the shell : $k$, free embryo capable of motion.

requires immediate washing, as otherwise infection may take place.

Treatment.-Santonin is the principal remedy for combating oxyuriasis. It is given in the same way as de- 
scribed in the treatment of ascaris lumbricoides. Here, however, irrigations of the bowel with water and the addition of a few drops of benzene or thymol or vinegar (three to four tablespoonsful to a quart), or of sapo medicatus in a one-half to one-per-cent solution may be advantageously used. The anal region should be thoroughly cleansed. If the pruritus ani is quite intense, application to the anal region and rectum of unguentum hydrarg. cinerei or the use of a suppository of ung. hydrarg. cinerei $1 \mathrm{gm}$, in cacao butter $2 \mathrm{gm}$. will afford relief.

Anchylostoma Duodenale. Dochmius Duodenalis or Strongylus Duodenalis. - This important parasite was first described by Dubini ${ }^{1}$ in 1838. Bilharz ${ }^{2}$ and Griesinger ${ }^{3}$ recognized this parasite as the cause of the Egyptian chlorosis. Some time afterward the anchylostoma was observed in severe cases of anæmia among workmen in tunnels and brickmakers.

The anchylostoma duodenale is cylindrical in shape, 0.5 to $1 \mathrm{~mm}$. thick and 6 to $18 \mathrm{~mm}$. long. It is yellowish or grayish-white in color, with translucent edges. The male is much shorter than the female. The cephalic end is curved toward the dorsal surface and is provided with an oral capsule at the margin of which there are six looklike teeth. Further within the capsule there are three sharp chitinous processes (Figs. 60 and 61). The male is more slender and transparent than the female. Its head end is bent backward. The tail end appears somewhat swollen, containing the bursa copulatrix, and is much more curved than the head. In the female the caudal end is pointed and armed with an awl-like prong; the genital opening

\footnotetext{
1 Angelo Dubini: Gaz. med. Lombard., 1843.

2 Bilharz: Wiener med. Wochenschr., 1856.

${ }^{3}$ Griesinger: Arch. f. physiolog. Heilkunde, 1854.
} 


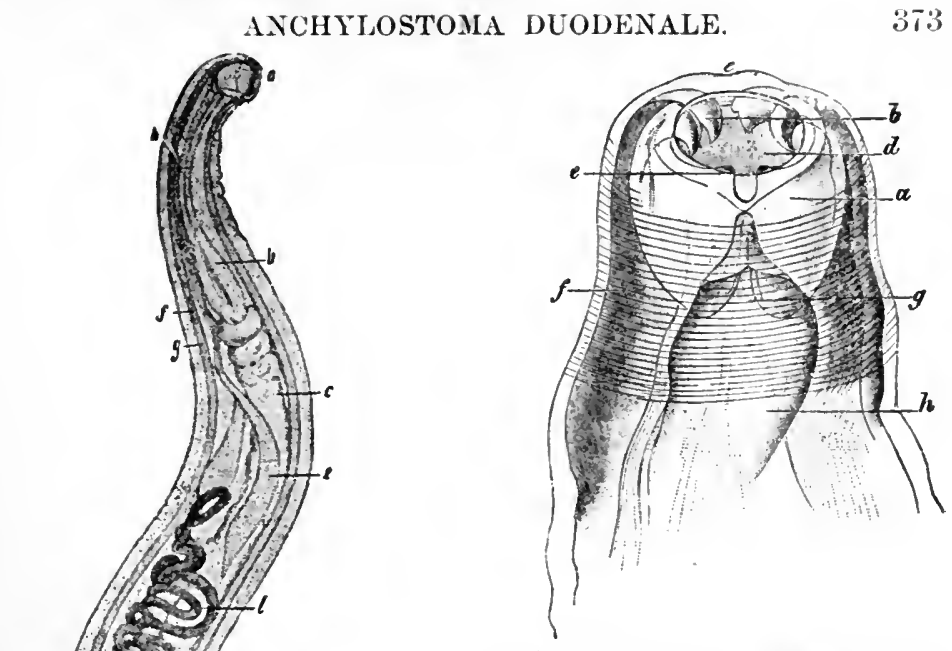

F1G. 61.-Cephalic End of Anchylostoma Duodenale. (Schultheiss.) $a$, Mouth-capsule; $b$, teeth of ventral border: $c$, teeth of dorsal border; $d$, buecal eavity: $\ell$, skin-sac on ventral side of head; $f$, muscular layer: $g$, dorsal groove; $h$, oesophagus.
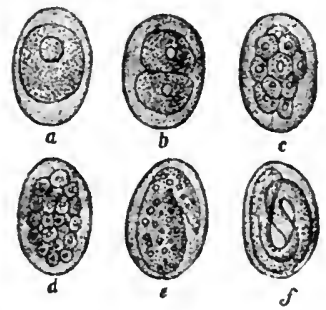

Fig. 6\%-Eggs of Anchylostoma Duodenale. (Perroncito and Schultheiss.) $a, b, c, d$. Different stages of eleavage: $\epsilon, f$, eggs with embryos. Magnifled 300 diameters.

Fig. 60.-Male of Anchylostoma Duodenale. (Schultheiss.) a, Head with month-capsule; $b$, oesophagus; $c$, intestine; $d$, anal glands; $e$, cervical glands ; $f$, skin; $g$, muscular layer; $h$, porus excretorius ; $i$, triple bursa ; $k$, ribs of the bursa; $l$. testleular canal ; $m$, vesicula seminalis ; $n$, ductus ejaculatorius ; 0 , groove of latter: $p$, penis; $q$, sheath of penis. Magnifled 20 diameters. 
lies behind the centre of the body. The eggs are oval, $0.06 \mathrm{~mm}$. in length and $0.03 \mathrm{~mm}$. in width (Fig. 62).

The habitat of the anchylostoma is the duodenum, the jejunnm, and the upper part of the ileum. Here the worm attaches itself to the intestinal mucosa and feeds by sucking the blood of his host. According to Leichtenstern,' active migration of the worm begins at the time of the first copulation in the fifth week. Young worms change their place quite frequently and hence gire rise to repeated hemorrhages. Colic, and acute anæmia are encountered at an early period after infection.

Under favorable conditions the eggs develop outside of the body into rhabditis-like larve, becoming enclosed in a protecting envelope or encysted. In this stage the larvæ may be carried along with the dust and contaminate fruit and water. On reaching the small intestine they develop into mature worms. This parasite is always encountered in great numbers if present in the intestines. Leichtenstern never found them in a smaller number than one hundred, but sometimes their total reached three thousand.

The symptoms produced by anchylostoma consist of gastralgia, nausea, occasionally romiting, constipation, rarely diarrhœa, and serere anæmia, the latter becoming progressively worse. The patient with anchylostoma does not greatly emaciate, but becomes pale, extremely weak, and suffers from dizziness and shortness of breath after the slightest exertion. His extremities are cold, slight hemorrhages occur frequently, and oedema of the ankles develops. A systolic murmur may be heard at the apex of the heart, the pulse is accelerated, and fever may be present

'Leichtenstern: Centralbl. f. klin. Medicin, 1885, and Deutsche med. Wochenschr., 1885, 1886, 1887. 
toward evening. An jnclination to eat earth (geophagia) is not rarely observed.

The dejecta are of a brownish color, although admixture of blood cannot be recognized macroseopically. Microscopically Charcot-Leyden's crystals, as well as the eggs of the parasites, are often found in the stools. The urine rarely contains albumin, but frequently indican. The condition of the blood resembles that found in pernicious anæmia: enormons decrease of the red blood corpuscles, poikilocytosis, nucleated red blood corpuscles, and a slight increase of the leukocytes, especially of the eosinophile cells.

Anatomically the mucosa of the small intestine is found greatly congested and ecchymoses are visible here and there. Peyer's patches and the solitary follicles are often swollen. The heart is found hypertrophied and dilated, the liver and spleen may be diminished in size, normal, or in an amyloid condition. The same can be said of the kidneys. There is no doubt that the principal deleterious action of the anchylostoma consists in the profuse loss of blood caused by the parasites. Whether some toxic substances generated by them participate in producing the grave symptoms is questionable.

The course of the disease is protracted and its severity depends greatly upon the number of parasites present. If the latter is great, the disease may progress quickly and the patient succumb with the symptoms of general dropsy, dyspnoea, and heart failure or pulmonary œedema. If the number of the parasites is small, the patient may live many years and ultimately recorer entirely. Recovery is also possible by successful expulsion of the parasites from the intestinal tract.

The diagnosis of anchylostomiasis is made by the pres- 
ence of the symptoms of anæmia in conjunction with the discovery of the anchylostoma eggs in the dejecta.

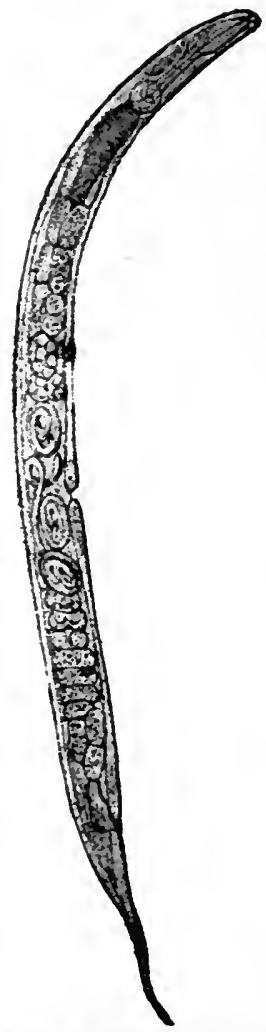

FIG. 63.-Female of Angulllula Stercoralis, with Eggs and Embryos. (Perronclto.) Magnifled 85 diameters.

With regard to prophylaxis the abore giren rules for the prevention of the round-and thread-worms are also applicable here. Extreme cleauliness of the body and of the food is of greatest importance.

The treatment consists in the administration of extract of male-fern, which should be employed in the same manner as described above for the tapeworm disease.

Anguillula Stercoralis. -This nematode is 0.8 to $1.2 \mathrm{~mm}$. long, the male shorter than the female (Fig. 63). The male is indigenous in Cochin China and Italy. In the latter country it often occurs simultaneously with anchylostoma. If the worms exist in large numbers they may produce pathological conditions. According to Golgi and Monti,' the anguillula stercoralis penetrates into Lieberkuehn's crypts and there deposits its eggs and young.

Anguillula intestinalis, which is $\mathbf{2 . 2 5}$ $\mathrm{mm}$. long, belongs to the same variety as anguillula stercoralis and is found under the same conditions. Only the female of this worm is known. The eggs develop in the intestinal canal and exhibit only the first stages of segmentation at the time of their passage with the frees.

${ }^{1}$ Golgi e Monti: Arch. per le science med., 1886, No. 3. 
Trichocephalus Dispar. Whip-Worm.-This parasite is quite common, but comparatively harmless. Its habitat is the cæecum and the neighboring section of the intestine. It lives upon blood which it abstracts from the intestinal mucosa. This parasite is $4-5 \mathrm{~cm}$. long, the male being smaller than the female. The head end, which is about three-fifths of the entire length, is drawn out into a fine thread; the tail end is not so thin, being up to $1 \mathrm{~mm}$. in thickuess (Fig. 64). Tho male has a spiral body from the end of which the spicule projects. The body of the female is straight and terminates in a blunt extremity. The

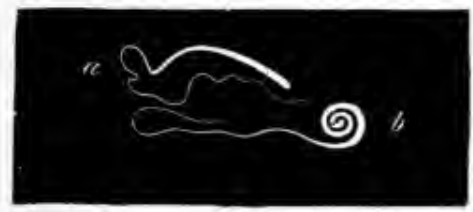

FIG. 64.-Trichocephalus Dispar. (Heller.) $a$ Female, $b$, male. Natural size.

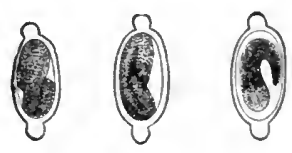

FIG. 65.-Ova of Trichocephalus Dispar in Process of Development. (Huber.)

ova are almost lemon-shaped, dark brown in color, 0.05 $\mathrm{mm}$. in diameter (Fig. 65). The number of eggs in a single female was estimated by Lenckart at 58,000. They are hatched out very slowly.

Lenckart asserts that the dispersion of the eggs and conseqnent spread of infection may readily occur through wind, rain, or dust, and that the eggs may be ingested with fruit and salads. The number of these worms found in one patient is usually small, from six to twenty.

The symptoms are but very slight, occasionally diarrhoea exists, sometimes there are some reflex nervous conditions.

The diagnosis can usually be easily made from the shape of the ova. The passage of the living worms in the stools occurs but rarely. 


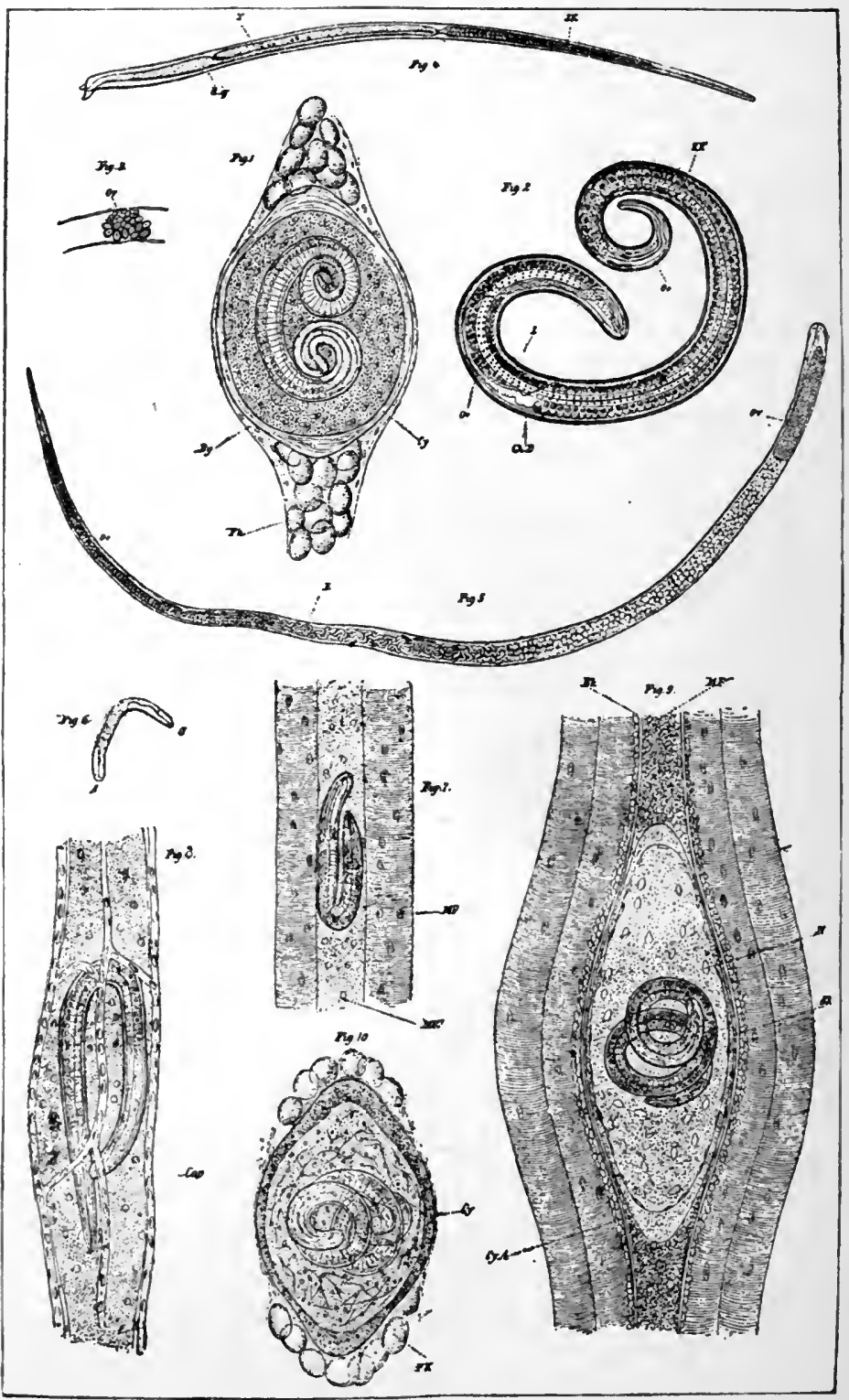

Plate I.-Trichina Spiralis (Huber). 
With regard to treatment Lutz recommends the administration of thymol; Mosler ${ }^{1}$ and Peiper ${ }^{2}$ employ rectal irrigation of water, to which a few drops of benzene have been added. Extract of male-fern may also be used internally.

Trichina Spiralis. - The trichina spiralis was discovered by Paget, ${ }^{3}$ but its pathological importance was first recognized by Zenker." This parasite is observed in two forms, the trichina of the intestine and the trichina of the muscles (see Plate I.).

The trichina reaches the stomach through the ingestion of pork containing encapsulated trichinæ. In the stomach the capsule opens about three to four hours after the inges-

'Mosler: "Darminfusion." Real-Encyclopädie der gesammten Heilkunde, Bd. v.

"Peiper: "Helminthen." Real-Encyclopädie der gesammten Heilkunde, Bd. ix.

${ }^{3}$ Paget, cited after Huber: "Twentieth Century Practice of Medicine," vol. viii., p. 608.

+Zenker: Deutsches Arch. für klin. Medicin, i., 1866.

Explasation of Plate I.

FIG. 1.-Muscle Trichina Enclosed in a Fully Developed Cyst. $\times 240 . \quad$ Cy, eyst; Bg, connective-tissue envelope; $k \boldsymbol{k}$, fat globules.

Fig. 2.-The Same Remored from the Cyst. $\times 400$. Oe, (Esophagus; $Z k$, cell bodies; $L$, side lines: $O v$, ovary: Ch.D, chyle duct.

FIG. 3. - Part of the Ovary. $\times 600$. Is readlly distinguisted from the testicle by the varying size of the germ cells.

Fig. 4.- - Iale Intestinal Triclina. $\times 100 . T$, Testicle; $d e j$, ejaculatory duct; $Z k$, cell bodies.

Fig. 5.-Female Intestinal Trichína. $\times 90 . O v$, ovary; $E$, embryos; $O e$, genital opening from whlch the embryos escape.

FIG. 6. - Free Embryo. $\times 410$. O, mouth; $d$, anus.

FIG. 7.-Finbryo About Three lays After Having Entered the Nuscle Fibre. MF , normal muscle flbre.

FlG. 8.-Muscle Trichina, About Six Days Old, in the Greatly swollen Sarcolemma Sheath Traversed by Capillary Vessels, Cap.

FiG. 9.-Nuscle Trichina, Four Weeks Old, Enclosed in a Capsule, $C y A$, within the sarcolemma sheath. $S k ; B k$, connertive-tissue capsule in process of active growth; $k$, nuclei ; $M F$. contents of the sarcolemma sheath at each pole of the capsule.

Fig. 10.-Muscle Trichina with Calcitted Capsule. $k k$, Fat globules. 
tion of the meat and the embryos rapidly develop. At the end of thirty to forty hours fructification of the young parasites takes place.

The intestinal trichinze are visible with the naked eye, the females being 3 to $4 \mathrm{~mm}$. long and the males half this size. The candal extremity is thicker than the head end. Five days after fecundation the females give birth to living young ones. The young brood wanders directly from the intestine of the host into his muscles. Here they further develop. In this condition they gire rise to a febrile disease accompanied by severe muscular symptoms which may lead to death. Sometimes the trichina become encapsulated. The symptoms vary according to the number of worms which have been ingested. Gastro-intestinal disturbances usually appear on the second or third day after the ingestion of the contaminated meat. Vomiting, diarrhoea, colic often appear.

The disease known as trichinosis, which depends upon the further development of the young embryos in the muscles of the host, is not within the scope of this book, and we refer to this parasite only as far as its occurrence in the intestines is concerned. With regard to prophylaxis pork should never be eaten raw. The treatment after the ingestion of trichinous meat consists in the employment of lavage of the stomach, if the physician is called early enough after the meal. In addition a vermifuge and cathartic remedy should be given immediately. 


\section{N D EX.}

Abelunasx, 22

Absorption as a function of the bowel, 24

Acholic stool, 58

Adenoma of tlie intestines, 167

Egineta, Paulus, 335

Albumin in the fæece, 53

Albuminates, putrefaction of, in the large intestiue, 21

Alimentation, rectal, $\pi$ subcutaneous, 7

Allingham, 37, 172, 185, 188

Allingham's rectal speculum, 37

Amoba, 349

Amœbic dysentery, 110

Amyloid ulcers, 140

Anacker, 311

Anæsthesia of the intestine, 333

of the rectum, 333

treatment, 334

Anatomy of the intestine, 1

Anchylostoma duodenale, 372

course, 375

diagnosis, 375

propliylaxis, 376

symptoms, 374

treatment, 376

Angioma of the intestines, 167

Anguillula intestinalis, 376

stercoralis, 376

Antiperistalsis of the intestine, 30

Anus, anatomy of the, 16

fissure of the, 193

Appendicitis, 196
Appendicitis, definition, 196

diagnosis, 214

differential diagnosis, 215

etiology, 197

general remarks, 196

morbid auatomy, 202

prognosis, 216

symptomatology, 206

synonyms, 196

treatment, 218

catarrhal, 202

indications for operation, 225

perforativa, 204

severe form, 204

ulcerosa et gangræenosa, 204

Appendicular inflammation, 196

Appendix rermiformis, 13

Aretaus, 110

Ascariasis, diagnosis, 367

proplyylaxis. 368

symptoms, 367

treatment, 368

Ascaris lumbricoides, 365 .

mystax, 369

Atony of the bowel, 291

Anscultation, 45

Awl-tail, 369

Balantidity coli, 351

Bamberger. 200

Barbacei, 200

Barthélemy, 114

Basch, 5i)

Bauhin's valve, 13

Bayle. 141 
Beauchef, 111

Beck, Carl, 198, 222

Benign tumors of the intestines, 167

Bernard, Claude, 19

Bienstock, 74

Bile pigment in the frees, 57

Bilharz, 372

Bilhalzia hæmatobia, 364

Biliary acids in the freces, 57

Birch-IIirschfeld, 144

Blood in the fieces, $\mathbf{5 6}$

Boas, J., 42, 48, 90, 212, 214, 219, $220,221,224,339$

Bönnecken, 248

Borborygmi, 45

Bothriocephalus cordatus, 359 latus, 357

Bonchard, 99, 298

Bourlet, 81, 263, 309

Bougies, rectal, 43

Bowel, atony of the, 291

Brahm-1Ioukgeest, 28, 283

Brintol1, 254

Brooks, LeRoy J., 267

Brunner's glands, 9

Brunton, 324

Bryant, J. D., 13, 151

Bull, W. T., 197, 223

Bunge, 59

Cacer, anatomy of the, 12

Cahn, 261

Cancer of the duodenum, symptoms, 159

of the intestine, 150

course, 163

definition, 150

diagnosis, 163

etiology, 150

location, 151

morbid anatomy, 152

prognosis, 164

symptomatology, 154

treatment, 164
Cancer of the large bowel, symptoms, 160 of the rectum, symptoms, 161 of the small intestines, symptoms, 160

Carbolydrates in the fæces, 54

Carbolic-acid injections in hemorrhoids, 186

Cash, 3:4

Catarrh, acute intestinal, 83 chronic, of the bowels, 94

Canterization in hemorrhoids, 186

Celsus, 110

Cercomonas intestinalis, 350

Cestodes, 351

Chareot, 286

Chlapowski, 40

Cholera nostras, 83

Clapotage, 42

Clark, Alonzo, 220, 302

Colic, intestinal, 326 mucous, 335

Colitis, acute, 90

Colon, anatomy of the, 11 ascending, 14 descending, 15 transverse, 14

Compression of the intestine, 227

Concretions in the fxees, 59

Constipation, 291

definition, 291

dependent upon other dis. eases, 296

diagnosis, 302

etiology, 292

habitual, 291

prognosis, 304

prophylaxis, 304

symptomatology 297 ,

synonyms, 291

treatment, 304

dietetic, 305

mechanical, 306

moral, 305

Cooper, 187 
Cooper-Forster, 155

Cornil, 342

Councilman, 113, 115, 118

Credé, 279

Crushing in the treatment of hemorrhoids, 188

Cruveilhier, 171, 337

Curschmann, 258, 264

Cysts of the intestines, 167

DA Costa, 33\%, 338, 344

Damsch, 46

Dastre, 19

Deaver, 222

Demant, 282

Delafield, F., 101

Diarrhoa, 284

acute, 83

diagnosis, 289

dyspeptic, 287

etiology, 284, 288

membranous, 335

morning, 101

nervous, 284

prognosis, 289

stercoral, 288

symptomatology, 284,288

treatment, 289

tubular, 335

Diet, $\pi 4$

Dilatation of the sphincters in the treatment of hemorrhoids, 185

Distoma hæmatobium, 364 hepaticum, 362 lanceolatum, 363

Dochmius duodeualis, 372

Douglas' fold, 15

Doumer, 309

Dubini, 372

Dunin, 294

Duodenal ulcer, 128 course, 133 . definition, 128 diagnosis, 133 etiology, 128
Duodeual ulcer, morbid anatomy, 129 prognosis, 134 symptomatology, 131 synonyms, 128 treatment, 134

Duodenitis, acute, 89

Duodenum, anatomy of the, 1

Dutrouleau, 121

Dynamic ileus, 257

Dysentery, 110 amœbic, 110 complications, 123

course, 123

definition, 110

diagnosis, 125

etiology, 110

morbid anatomy, 115

prognosis, 125

symptomatology, 119

synonyms, 110

treatment, 125

Dyspeptic diarrhoea, 287

Epeboins, 198, 207, 208

Edwards, 338

Ehrlich, 338, 342

Ehrmann, 31

Eichberg, 113

Eichhorst, 131

Eisenlohr, 139

Electricity in intestinal obstruction, 263

in the treatment of constipation, 308

in the treatment of disease, 81

Elsberg, C. A., 70

Embolic ulcers, 13is

Embolus of the arteria mesaraica superior, 136

of the inferior mesaraic artery, 139

Endo-appendicitis, 203

Enemata in the treatment of con. stipution, 310 
Enteralgia, 326 definition, 326 diagnosis, 329 etiology, 326 prognosis, 330 symptomatology, 327 synonyms, 326 treatment, 330

Enteritis, acute, 83 chronic, 94 crouposa, 110 membranous, 335 necrotica, 110

Enterospasmus, 295

Erdmann, John F., 267

Escherich, 73,200

Esmarch, 189

Ewald, C. A. 62, 96, 131, 219, 220, $224,339,340$

Examination, methods of, 32

Extirpation of hemorrhoids, 189

FAces, abnormal admixtures in the, 51

albumin in the, 53

bile pigment in the, 57

biliary acids in the, $\mathbf{5 7}$

blood in the, 56

carbohydrates in the, 54

chemical examination of the, 52

concretions in the, $\mathbf{5 9}$

examination of the, 49

fat in the, 55

ferments in the, 59

fragments of tumor in the, 51 micro-organisms in the, $\mathbf{7 1}$

mieroscopical examination of the, 62

muein in the, 53

odor, 50

peptone in the, 54

propeptone in the, 54

pus in the, 51

reaction of the, 52
Fæces, remnants of food in the, 51 Fat in the frees, 55

Fecal accumulation as a cause of chronic obstruction, 273 fever, 302 tumors complicating constipation, 300

Ferguson, E. D., 233, 267

Fermentation test, Schmidt's, 55

Ferments in the fæces, 59 of the pancreas, 19

Fever, fecal, 302

Fibroma of the intestines, $\mathbf{1 6 7}$

Finger cot, 42

Fischel, 286

Fischl, 89

Fissure of the anus, 193

Fitz, Reginald, 114, 197, 222, 258

Flatan, 312

Flatulency, 321

Fleiner, 79, 262, 311, 346

Fleischer, 59, 214, 282

Fluke worms, 362

Foreign bodies, obturation by, 234

Fowler, 197, 199, 200, 202, 214, 222

Frentzel, 265

Frerichs, 141

Friedenwald, J., 43

Fürbringer, 265

GALLI stones, obturation by, 233

Galvano-cautery in the treatment of hemorrhoids, 188

Gerhardt, 137

Gerry, 114

Gersuny, 41

Gibson, C. L., 266

Glycerin injections in the treatment of constipation, 311

Golgi, 376

Good, Mason, 336

Gouley, J. W., 267

Graser, 189, 259, 264, 265

Grasse, 367

Graves, 220 
Grawit», 139

Griesinger, 3\%2

Grisolle, 196, 19\%, 220

Guttmann, P., 204

Gymnastic exercises in the treat. ment of constipation, 308 exercises in the treatment of disease, 80

Habersion, 79, 300

Habitual constipation, 291

Hackel, 309

Haguenot, 241

Hall, Marshall, 87

Hammarsten, 26

Harris, 113, 114, 116, 127

Haustra coli, 12

Hawkes, F., 223

Hegar, 139

Hemorrhoids, 169 complications, 189

definition, 169

diagnosis, 179

etiology, 169

morbid anatomy, 171

prognosis, 180

symptomatology, 174 .

synonyms, 169

treatment, 180

rarlical, 185

Henrot, 314

Heryng, 39

Heusgen, 155

Hippocratos, 110

Hirsehler, 22

Hlava, 113

Hodenpyl, 200

Hoffmann, 265

Houston, 186

Hoyer, 338

Huber, 356, 366, 370

Hydrocephaloid, acute, 87

Hydrotherapy, 80

in the treatment of constipation, 309
Hyperasthesia of the intestine, 333

Hypogastrie neuralgia, 332

treatment, 332

IleO-CACAl, valve, 13

Ileum, anatomy of the, 4

Ileus, 227

dynamic, 257

Illoway, $30 \%$

Incarceration, acute, 255

Inflation of the bowel with air in intestinal obstruction, 262

of the intestine, 45

Infusoria, 350)

Injection of water per anum for examination, 48

Injections as a method of treatment, 78

in the treatment of constipation, 310

Inspection, 34

Interrogation, 32

Intestiual catarrh, acute, 83 definition, 83

diagnosis, 91

duration, 90

etiology, 83

localization, 89

morbil anatomy, 8;

prognosis, 91

symptomatology, 86

synonyms, 83

treatment, 91

catarrh, ehronie, 94

course, 10:3

definition, 94

diagnosis, 103

etiology, 94

morbil anatomy, 95

symptomatology, 98

synonyms, 94

treatment, 105

colic, 326

neurasthenia, 347

diagnosis, 348 
Intest inal neurasthenia, treatment, 348

obstruction, 2206

obstruction, acute, 226

course, $24 \pi$

definition, 227

diagnosis, 249

etiologry, 228

loeation of the obstruction, 2.j1

objective signs, 245

pathological (hanges, 236

reengnition, 249

recognition of the different forms, 25.)

synuptomatology, 238

synonyms, 227

treatment, 258

treatment, medical, 258

treatment, surgical, 266

obstruction, chronic, 268

complications, 276

coulse: 277

diagnosis, $2 \pi$

etiology, 268

prognosis, $27 \%$

symptomatology, 269

treatment, $2 \approx 8$

treatment, surgical, 280

parasites, 349

vertigo, :301

Intestine, anatomy of the, 1

anfesthesia of the, $333: 3$

compression of the, 227

liyperasthesia of the, 33:3

obturation of the, 233 , 256

parzesthesia of the, 3333

strangulation of the, 228

Intestines, motor neuroses of the, 284

neoplasms of the, 150

nervous affections of the, 282

elassification, 283

paralysis of the, 314

diagnosis, 315
Intestines, paralysis of the, treatment, 315

peristaltic restlessness of the, 319

sceretory nemroses of the, 3335

sensory newroses of the, 326

ulcers of the, 128

Intussusception, 2:34, 256

agonai, 2:36

Irrigator, Kemp's rectal, 79

JAFFÉ, 253

v. Jaksch, 54

Jejunitis, acute, 89

Jejunum, anatomy of the, 4

Jürgens. 97

KAl1:, Arthur, 30s

Kartulis, 112, 113

Kelly, 37

Kelly's reetal speculum, 38

Kelsey, 186

Kemp's rectal irrigator, $; 9$

Kerkring's valves, 8

Kittagawa, $3: 39$

Klebs, 144

Klenfierer, 310, 311

Klubbe, 262

Koch, 112

Kocher, 264

Koenig, 269

Körte, 265

Kossobudiskj, 184

Kraus, 12!)

Kuhn, 44

Kundrat, 166

Kussmaul, 137, 261, 262, 309, 311, 346

LABOUJBÈNE, 337

Latleur, 113, 115, 118

Lambl, 112

Lange, F., 186, 192

Langenbeek, 188 
Large bowel, physiology of the, 18

structure of the, $1 \%$

intestine, anatomy of the, 11

Lavage of the bowel, 48

in intestinal obstruction, 262

of the stomach in intestinal obstruction, 261

Laveran, 124

Leguen, F., 222

Leichteustern, 67, 226, 228, 295, $367,368,370,374$

Lemazurier, 300

Lenander, 197

Leube, $7 \%, 134,801$

Leubuscher, 309

Leuckart, 356, 367, :377

Levi, 300

v. Leyclen, 214, :338, 342

Lieberkühn's glands, 9

Ligature in hemorrhoids, 187

Iimbourg, 22

Lindberger, 22

Lipoma of the intestines, 167

Litten, 136

Liver fluke, 362

Loesch, 112

Ludwig, 25

Lusk, 19, 26

Lutz, 367, 370, 379

Lympho-sarcoma of the intestines, 166

Macfadyen, 20, 72

Madelung, 167

Malmsten, 351 .

Narcy, II. O., 267

Matterstock, 201

Maggot-wor'm, 369

Mannaberg, 73

Massage in intestinal obstruction, 263

in the treatment of constipation. 306
Massage in the treatment of disease, 80

Massaiutin, 113

Massloff, 282

Nathews, 90

Nayer, 128

Nayor, A., 90

MclBumey, 41, 197, 223

McCosh, A. J., 223

Meckel's diverticulum, strangulation by, $2: 30$

Membranous diarrhoª, 335 enteritis, 335 definition, 335 diagnosis, 343 ("tiology, :38: history, 38it symptomatology, 341 synonyms. $333 \pi$ treatment, 344

Mendelson, Walter, 339

Nercury, metallic, in intestinal obstruetion. 26.5

Meteorism, 321 diagnosis, :323 etiology, 3:1 prognosis, $3: 32$ symptomatology, 322 treatment, 323

Meydl, 151

Meyer, Willy, 223, 224

Micro-organisms in the freces. 71

Niller, 29

Minich, 61

Minkowski, 28, 47

Miserere, 227

Monti, 376

Moreatu, 282

Morgagni, 336

Morris, R. T., 208, 222

Mosler, 379

Motion of the intestine, 28

Mucin in the freces, 53

Mucous colic, 33i5

Mï̈ller. Max, 1,2 
Munk, 25, 27

Murphy, 222, 280

Murphy's button, obturation by, 234

Musser, 113

Myoma of the intestines, 167

Nasse, 113

Nematodes, 365

Nencki, 19, 20, 72

Neoplasms of the intestines, 150

Nervous affections of the intestines, 282

diamboa, 284

Neuralgia, hypogastric, 332 mesenterica, 326

Neurasthenia, intestinal, 347

Neuroses of the intestines, motor, 284 secretory, 335\% sensory, 325

v. Noorden, C., 56, 344, 345

Nothnagel, 28, 30, i4, 138, 150, $166,197,202,214,217,220$, 224, $2335,269,283,285$, 294, 302, 338, 342

Nuttal, 72

Obstil'atio, 291

Obstruetion, intestinal, 226

Obturation of the intestine, 233, 256

Oil injections in the treatment of constipation, 311

Opium in intestinal olstruction, 259

Oppolzer, 330

Osler, 113

Oxyuris vermicularis, 369

diagnosis, 371

prophylaxis, 371

symptomatology, 370

treatment, 371
Palpation, 40

Panereatic juice, digestive power of, 19

Parasthesia of the intestine, 333

Paralysis of the intestines, 314 of the sphineters of the anus, 317 dliagnosis, 318 prognosis, 318 treatment, 318

Parameeium coli, 351

Parasites, intestinal, 349

Paratyphlitis, 196

Parenski, 13ji

Paresis of the splineters of the anus, 317

Pariser, 53, 339, 342

Passio iliaca, 227

Péan, 280

Peiper, 379

Penzoldt, 214, 219, 220, 224

Peptone in the faces, 54

Percussion, 44

Peristalsis of the intestine, 28

Peristaltic restlessness of the intestines, 319 definition, 319 diagnosis, 320 etiology, 319 symptomatology, 319 treatment, 320

Perityphlitis, 196

Petriquin, 220

Pettenkofer, 57

Peyer, 285

Peyer's patches, 11

Ptlinger, 30

Phlebectasia hemorrhoidalis, 169

Pighead, 357

Piles, 169 arterial, 172 cetpillary, 172 venous, 173

Pilliet, 139

Pin-worm, 869 
Plica Douglasii, 15

Poelchen, 145

Polypi of the intestines, 167

Pooley, 186

Proctitis, 90

Proctoseopy, 37

Proetospasmus, 316

diagnosis, 317

treatment, 317

Prolapse of the rectum, 189

Propeptone in the freces, 54

Protozoa, 349

Pumeture of the bowel in intestinal obstruction, 264

Purgatives in the treatment of constipation, 312

Putrefaction of albuminates in the large intestine, 21

Qนтаке, 50, 113, 282

RACuForD, 19

Rectal alimentation, 77

bougies, 43

electrole, 81

specula, 37

Rectum, anesthesia of the, $3: 33$

anatomy of the, 15

prolapse of the, 189

Reichmann, 39

Ribbert, 198

Riedel, 202, 224

Rieder, 66

Roentgen rays in examination of the bowel, 39

Romberg, 33:

Rose, A., 263, 307

Rosenbach, 46, 247

Rosenheim, 102, 167, 308, 315, 319. 32:3, 347

Rosenstein, 25, 27

Ross, 113

Rotlımann, 338

Rotter, 197

Round-worms, 365
Roux, 186, 197

Rubner, 27

Ruedi, 297

Runeberg, 46

Rushmole, J. D., 267

SAll, $197,217,220,307,324$

Salmon, 187

Salvioli, 25

Sands, 197

Sarcoma of the intestines, 166

Sasaki, 97

Schiff, 28

Schmidt, 54, 58

Sehmilt's fermentation test, 55

Selımidt-Mïhlleim, 25

Schmitz, 22

Schnetter, 46

Schoening, 150

Srhuberer. 113

Seolecoiditis, 196

Seat-worm, 369

Secretory function of the intestines, 18

neuroses of the intestines, $33: 35$

Senator, 302

Senn, 26:3

Sennertius, $3: 36$

Sicbar, 20 , $72^{\circ}$

Sigmoid flexure of the colon, 15

Simoll, 43

Sims, 37

Sims' rectal speculum, 37

Siredey, F., 336, 344

Small intestine, anatomy of the, 4 st ructure of the, 6

Sollé, 111, 116, 126

Solitary follicles of the intestines, 10)

Somenburg, 197, 214, 224

Spasm of the rectum, 316

Spasmodic contraction of the bow(l), 295

Specula, rectal, $: 37$

Spool-worm, 365 
Sporozoa, 350

S romanum, 15

Starke, 129

Stein, R., 224

Stengel, 113

Stercoral diarrhœa, 288

Stewart', 267

Stockton, 113

Stokes, 220

Stool, acholic, 58

Strangulation of the intestine, 228

Stricture of the rectum as a cause of chronic obstruction, 275

Strongylus duodenalis, 372

Subcutaneous alimentation, 77

Sutton, E. M., 47

Syms. Parker, 267

Syphilitic ulcers, 144

Thena cucumerina, 358

diminuta, 359

flavopunctata, 359

lata, 35\%

mediocanellata, 35is

nana, 358

Saginata, 3is

solium, 354

Tæniæ of the large intestine, 12

Talamon, 197

Tapeworms, 351

prophylaxis, 359

treatment, 359

Tavel, 200

Thermocautery in the treatment of hemorrhoids, 188

Thierfelder, 72

Thread-worm, 369

Thrombotic ulcers, 135

Thrombus of the mesenteric veins, 139

Toxic ulcers, 145

Transillumination of the bowel, 39

Trastour, 262

Treatment, methods of, 74

Trematodes, 362
Trematodes, treatment, 365

Treves, 192, 214, 236, 240, 259. 262, $264,266,268,270,271,281$

Trichina spiralis, 379

Trichinosis, 380 symptoms, 380

prophylaxis, 380

treatment, 380

Trichocephalus dispar, $37 \pi$ diagnosis, $37 \%$ symptonss, 377 treatment. 379

Trichomonas intestinalis, 350

Trousseau, 284

Tuberculous ulcers, 141

Tubular diarrhoea, 335

Tympanites, 321

UlCEr, duodenal, 128

Ulcers, amyloid, 140

embolic, 135

of the intestines, 128

syphilitic, 144

thrombotic, 13.5

toxic, 14:

tuberculous, 141

Urobilin in the freces, 57

VaLve, ileo-crecal, 13

of Bauhin, 13

Valvule comniventes Kerkringi, 8

Van Cott, 199, 201

Vermes, 351

Vermiform appendix, 13

Verneuil, 185

Vertigo, intestinal, 301

Virchow, 140

Voit, 19, 24, 26

Volkmann, 162

Volvulus, 232, 255

Volz, 201, 220

WAlLACE', 319

Weber, L., 108

Weigert, 338 
INDEX.

Weir, $19 \tau$

Wertheimer, 330

Whip-worm, $3 \pi 7$

Whitelead, 189, 336, 344

Wiggin, Fred. H., 267

Willigk, 129

Wilson, 200

Wolf, H. J., 224
Woodward, $83,96,269,292$, 336

Worms, intestinal, 351

ZeNKer, 379

Ziemssen, 45, 263, 264

Zuckerkandl, 198

Zunker, 350 





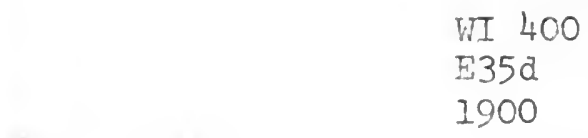

Eihorn, llax

1900

Diseases of the intestines. . .

\section{MEDICAL SCIENCES LIBRARY UNIVERSITY OF CALIFORNIA, IRVINE IRVINE, CALIFORNIA 92664}


